\title{
AN INVESTIGATION OF BINARY CODED EXCITATION METHODS USING GOLAY CODE PAIRS FOR HIGH FREQUENCY ULTRASOUND IMAGING
}

By

\section{Xuegang Su}

Master of Applied Science, Beijing Institute of Technology, Beijing, 1997

Bachelor of Applied Science, Beijing Institute of Technology, Beijing, 1992

\author{
A Thesis \\ Presented to Ryerson University \\ In partial fulfillment of the \\ requirements for the degree of \\ Master of Applied Science \\ In the Program of
}

Electrical and Computer Engineering Department

Toronto, Ontario, Canada, 2004

CC Xuegang Su 2004 
UMI Number: EC52980

\author{
All rights reserved \\ INFORMATION TO USERS
}

The quality of this reproduction is dependent upon the quality of the copy submitted. Broken or indistinct print, colored or poor quality illustrations and photographs, print bleed-through, substandard margins, and improper alignment can adversely affect reproduction.

In the unlikely event that the author did not send a complete manuscript and there are missing pages, these will be noted. Also, if unauthorized copyright material had to be removed, a note will indicate the deletion.

\title{
UMI
}

UMI Microform EC52980

Copyright 2009 by ProQuest LLC

All rights reserved. This microform edition is protected against unauthorized copying under Title 17, United States Code.

ProQuest LLC

789 East Eisenhower Parkway

P.O. Box 1346

Ann Arbor, MI 48106-1346 


\section{Author's Declaration}

I hereby declare that I am the sole author of this thesis.

I authorize Ryerson University to lend this thesis to other institutions or individuals for the purpose of scholarly research.

Signature:

I further authorize Ryerson University to reproduce this thesis by photocopying or by other means, in total or in part, at the request of other institutions or individuals for the purpose of scholarly research.

Signature: 


\section{Borrower's Page}

Ryerson University requires the signatures of all persons using or photocopying this thesis. Please sign below, and give address and date. 


\begin{abstract}
AN INVESTIGATION OF BINARY CODED EXCITATION METHODS USING GOLAY

CODE PAIRS FOR HIGH FREQUENCY ULTRASOUND IMAGING

Xuegang Su, Master of Applied Science

Electrical and Computer Engineering Department, Ryerson University, Toronto, Ontario, Canada, 2004
\end{abstract}

We are investigating the feasibility of binary coded excitation methods using Golay code pairs for high frequency ultrasound imaging as a way to increase the signal to noise ratio. I present some theoretical models used to simulate the coded excitation method and results generated from the models. A new coded excitation high frequency ultrasound prototype system was built to verify the simulation results. Both the simulation and the experimental results show that binary coded excitation can improve the signal to noise ratio in high frequency ultrasound backscatter signals. These results are confirmed in phantoms and excised bovine liver. If just white noise is considered, the encoding gain is $15 \mathrm{~dB}$ for a Golay pair of length 4 . We find the system to be very sensitive to motion (i.e. phase shift) and frequency dependent (FD) attenuation, creating sidelobes and degrading axial resolution and encoding gain. Methods to address these issues are discussed. 


\section{Acknowledgements}

I would like to thank my supervisor, Dr. Michael Kolios whose intellectual support and guidance were integral to the completion of this project.

I would like to thank the other researchers and students including Arthur Worthington

and Xia $\mathrm{Wu}$ in the Princess Margaret Hospital, Yuexi Huang at the SuunyBrook Hospital and, Shuxue Quan at the SONY Corporation, Neeta Parmar who wrote some simulation software, Peter Chun, Noushin Farnoud and in our lab. I appreciate greatly of all their help.

Finally, I would like to thank all my professors in the Electrical and Computer Department and the Physics Department for all their help and Ryerson University for providing the facilities and environment that makes research such a wonderful experience. 


\section{Dedication}

This thesis is dedicated to my parents, my wife, and my son for all their love and support. 


\section{Table of Contents}

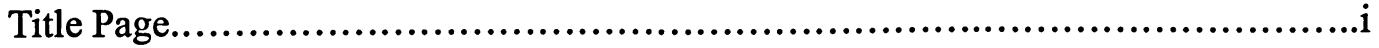

Author's Declaration..................................................................

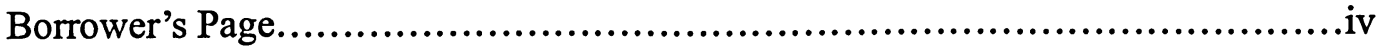

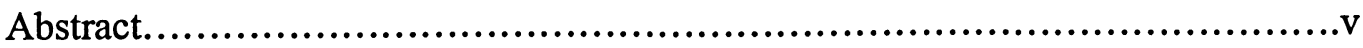

Acknowledgements.............................................................

Dedication..........................................................................

Table of Contents.....................................................................

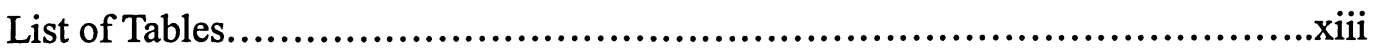

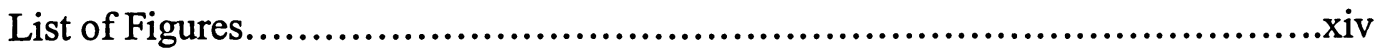

Chapter 1 Introduction........................................................

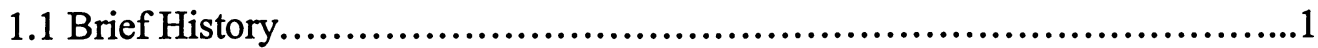

1.2 Statement of Problem and Organization of Thesis...............................3

1.3 Nature of Ultrasound........................................................

1.4 Wave Fundamentals...................................................6

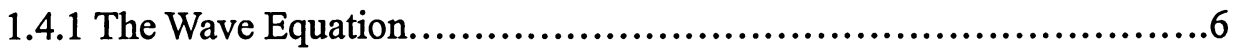

1.4.2 The Acoustic Properties of Media .......................................7

1.4.3 Reflection and Refraction ..........................................

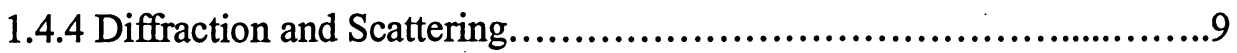

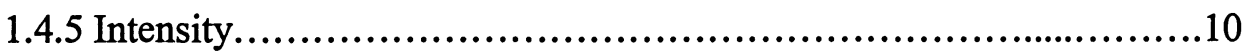

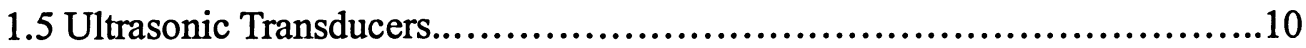

1.5.1 Parameters and Materials...........................................11

1.5.2 Focusing field...................................................... 12

1.6 Velocity, Attenuation and Absorption ......................................14

1.6.1 Velocity of Sound in a Medium..........................................14

1.6.2 Attenuation of Biological Medium.....................................15

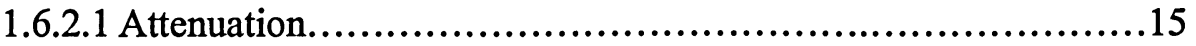

1.6.2.2 Frequency Dependent Attenuation................................17

1.6.3 Absorption of Medium...............................................19

1.7 Scattering by Biological Materials....................................20 
1.7.1 Introduction......................................................20

1.7.2 Scattering Strength ...............................................22

1.7.3 Pulse Echo Response................................................23

1.7.4 Single Particle Model...................................................24

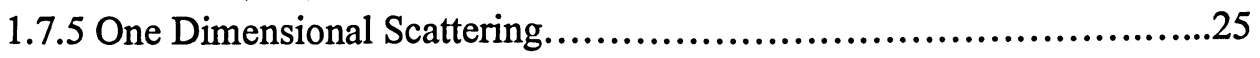

1.7.6 Our Research Interest..............................................27

1.8 Ultrasound System....................................................29

1.8.1 The Structure of an Ultrasound System................................29

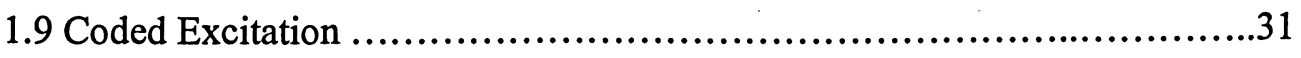

1.9.1 Introduction.......................................................... 31

1.9.2 The Theory of Coded Excitation......................................32

1.9.3 Two Coded Excitation Methods.........................................35

1.9.3.1 FM Chirp Coded Excitation....................................35

1.9.3.2 Binary Coded Excitation ...........................................39

1.9.4 Properties of Golay code and Golay code paris .........................40

1.9.5 The Components of a Binary Coded Excitation System.................43

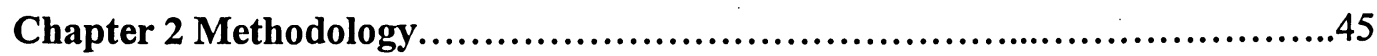

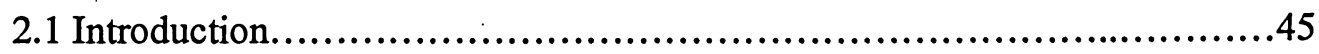

2.2 Simulation of Coded Excitation..............................................45

2.2.1 Encoded Signal...................................................46

2.2.1.1 Golay Codes....................................................46

2.2.1.2 Code Generation...............................................47

2.2.1.3 Modulated Signal Type.........................................51

2.3 Backscatter Signal.................................................5

2.3.1 One Dimensional Scattering Model....................................54

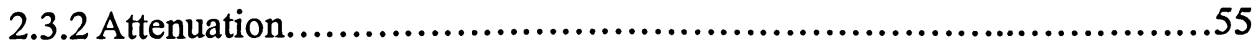

2.3.2.1 Frequency Independent Attenuation.............................55

2.3.2.2 Frequency Dependent Attenuation...............................56

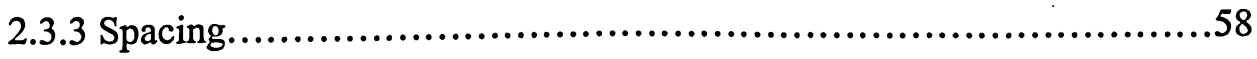


2.3.4 Processing Coded Backscatter Signals..................................59

2.3.4.1 Reflection Reference Processing Method........................59

2.3.4.2 Code Reference Processing Method............................60

2.3.4.3 Code with Phase Reference Processing Method.................62

2.3.5 Simulations........................................................64

2.3.6 Evaluation Methods.................................................65

2.3.6.1 Signal to Noise Ratio............................................66

2.3.7 Signal Distortion...................................................69

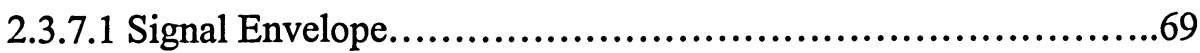

2.4 Experimental Prototype of Binary Coded Excitation System.................70

2.4.1 The Experimental System...........................................71

2.4.2 Two Interesting Phenomena.........................................72

2.4.2.1 Transducer Focus................................................72

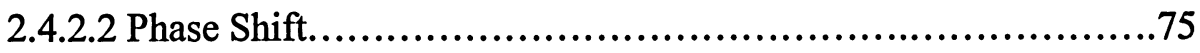

Chapter 3 Results..............................................................

3.1 Introduction.........................................................

3.2 Theoretical Simulation Results for the Binary Coded Excitation ...............77

3.2.1 Sparse Spacing Models.............................................77

3.2.1.1 Pulse Compression without Attenuation..........................78

3.2.1.2 Pulse Compression with Plain Attenuation.......................80

3.2.1.3 Frequency Dependent Attenuation................................83

3.2.1.4 Effect of Code Length using Sparse Spacing Models...............85

3.2.1.5 Impulse Response for the Coded Excitation Method...............86

3.2.2 Dense Spacing Models.................................................86

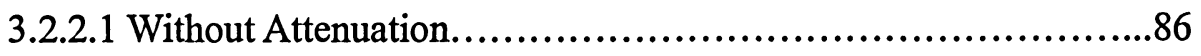

3.2.2.2 Plain Attenuation..............................................89

3.2.2.3 Frequency Dependent (FD) Attenuation..........................90

3.2.2.4 Simulations with closely spaced scatterers......................92

3.3 Experimental Results for the Binary Coded Excitation System................94 
3.3.1 Three Processing Methods...............................................95

3.3.2 Signal Encoding Gain ................................................98

3.3.3 Impulse Response for Our Binary Coded Excitation System ...........100

3.3.3.1 The Phase Shift Effect..........................................102

3.3.3.2 The Effect of Code Length.....................................103

3.3.4 Results from Phantoms...........................................105

3.3.4.1 The Effect of Code Length.....................................106

3.3.4.2 The Effect of Depth.............................................109

3.3.5 Critical Depth for our System ..........................................111

3.4 Backscatter Signal from Real Tissue..........................................115

3.4.1 Different Processing Methods......................................116

3.4.2 Phase Shift......................................................117

3.4.3 Similarity of Signals..............................................119

3.4.4 The Gain in Signal to Noise Ratio...................................120

Chapter 4 Conclusions and Future Work........................................122

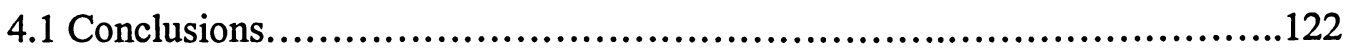

4.1.1 Feasibility of Binary Coded Excitation Method.....................122

4.1.2 The Mechanism of the Binary Coded Excitation Method.............123

4.1.3 The Effect of Code Length........................................125

4.1.4 Phase Shift Sensitivity............................................125

4.1.5 Impulse Response and Similarity of Signal........................127

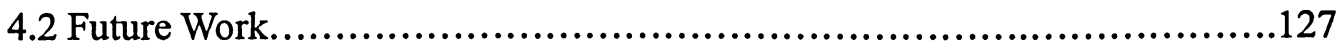

Appendix A System Design............................................130

A1.1 Arbitrary Waveform Generator...........................................130

A1.2 Power Amplifier......................................................136

A1.3 Signal Amplifier..................................................137

A1.4 Oscilloscope.....................................................138

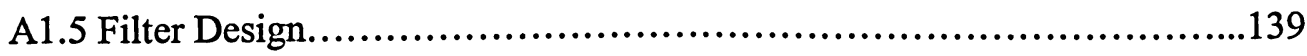




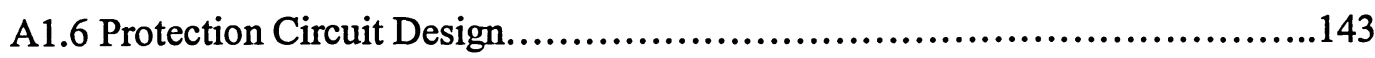

A1.7 Transmission Line Impedance Matching................................145

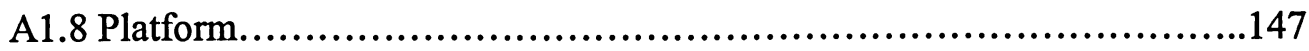

A1.9 Connection Adapter and Cables.........................................147

A1.10 Two Challenges of the Lab System....................................149

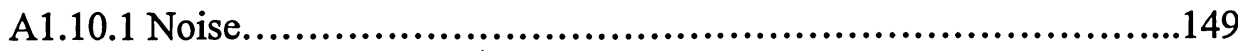

A1.10.2 Inertia of Transducer.........................................150

Appendix B Noise Reduction for the Power Amplifier........................152

Appendix C Transducer Equivalent Circuit and Impedance matching..........154

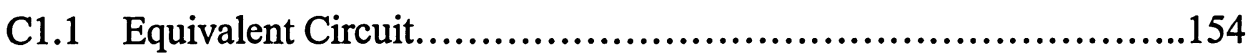

C1.2 Impedance Matching..........................................156

C1.2.1 Electrical Impedance Matching................................156

C1.2.2 Mechanical Impedance Matching.............................157

Appendix D Biological Effects..........................................158

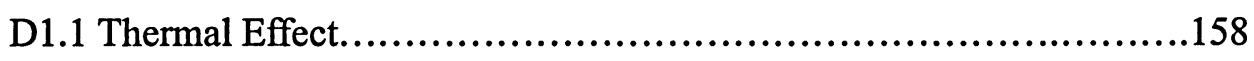

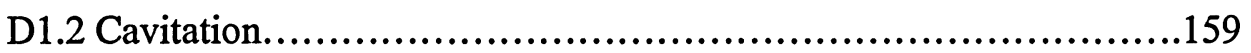

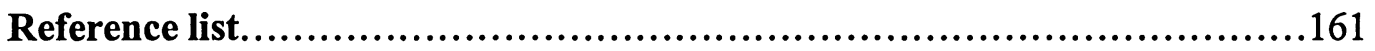

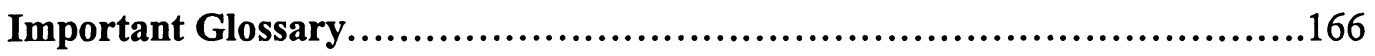

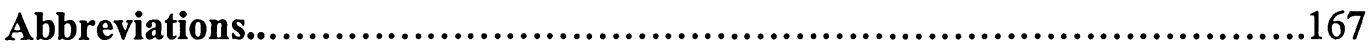




\section{List of Tables}

1.1 Characteristic Acoustic Impedances of Selected Media...........................8

1.2 Approximate Attenuation Values for Human Tissue...........................16

1.3 Contributions of Scattering to the Attenuation Coefficient..........................22

2.1 Results of Element Pairing for Sequence 1 and Sequence 2.....................48

3.1. Encoding Gains for Different Depths with Plain Attenuation....................82

3.2 Encoding Gain for Different Depths with FD Attenuation.......................84

3.3 The Relation between Code Length and Gain...................................89

A1.1 The Predefined Variables................................................133

A1.2 Power Amplifier Specifications.........................................136

A1.3 Specifications of the Signal Amplifier.......................................138

A1.4 The Line Lengths for Maximum Pulse Echo Amplitude........................144 


\section{List of Figures}

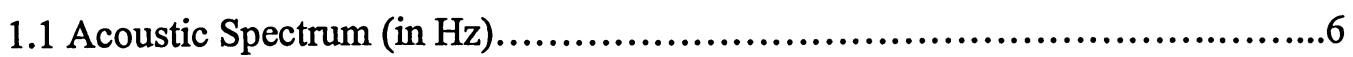

1.2 Schematic of Transducer....................................................

1.3 The Speed in Pure Water at $5 \mathrm{MHz}$ with Temperature $0-90 \ldots \ldots \ldots \ldots \ldots \ldots \ldots \ldots \ldots . .15$

1.4 Tissues' Frequency Dependent Attenuation Coefficient .........................18

1.5 Ultrasound Images of $13^{\text {th }}$ Week Fetus..........................................21

1.6 Simplified Representation of the Echo Response.................................24

1.7 Single Particle Model of Scattering...........................................25

1.8 One Dimensional Scattering Model..........................................27

1.9 Normal-Treated-Normal Pellet of Cells Using the $20 \mathrm{MHz}$ (A) and $40 \mathrm{MHz}$ (B)

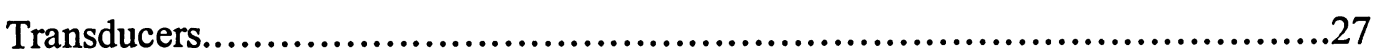

1.10 A Lymphoma Tumor in Mouse Leg imaged at 40MHz........................28

1.11 The Structure of Ultrasound System.........................................29

1.12 Conventional Pulse and Coded Excitation.....................................32

1.13 Conventional Systems and Coded Excitation Systems.........................34

1.14 Encoded Method and Autocorrection..........................................35

1.15 FM (Frequency Modulated) Chirp........................................36

1.16 Implementation of FM Chirp..........................................37

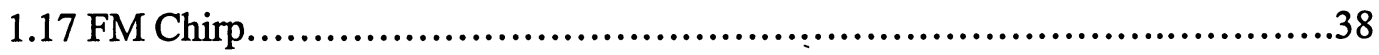

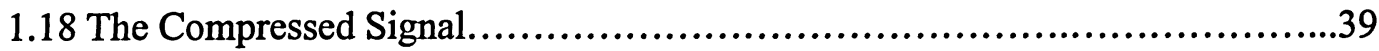

1.19 Binary Coded Excitation...................................................39

1.20 Golay Code and Its Autocorrelation...........................................41

1.21 Golay Code Pairs............................................................

1.22 Golay Coded Excitation Signal and Conventional Signal.......................42

1.23 Coded Excitation System...................................................44

2.1 The Structure of Simulation.................................................45

2.2 Golay Code Pairs.........................................................48

2.3 Long Coded Signals in Focused and Unfocused Transducer......................50

2.4 (a) Emitted Signal from Transducer (b) Simulated Signal........................51 
2.5 (a) The Second Modulated Signals (b) Reference Modulated Signal...............52

2.6 Third type of Modulated Signals..............................................53

2.7 Attenuation in Frequency Domain...........................................56

2.8 Implementation FD of Attenuation.........................................58

2.9 Simulation Results with Different Spacing.....................................59

2.10 Reflection Reference Processing Method....................................60

2.11 Code Reference Processing Method............................................61

2.12 Results from One Cycle Code Reference and Reflection from One

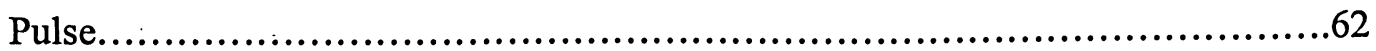

2.13 Code with Phase Reference for Cross Correlation..............................63

2.14 Schematic diagram of the Program...........................................65

2.15 Noise Type and Penetration Depth $\times$ Frequency.............................66

2.16 A Real Backscatter Signal from Liver.......................................68

2.17 Getting Envelop of Signal by Hilbert Transform..............................70

2.18 The Structure of Binary Coded Excitation.....................................70

2.19 Prototype Binary Coded Excitation System...................................72

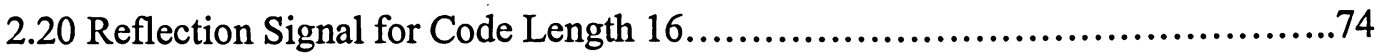

2.21 Focus Point for Reflection Signal of Code Length 16..........................74

2.22 Phase Shift between Golay Pairs...........................................75

2.23 No Phase Shift after Platform is Stabilized.....................................76

3.1 (a) One Cycle Code Reference for Processing (b) Golay Coded Signal.............78

3.2 Backscatter Signal from Sparse Spacing without Attenuation......................79

3.3 Encoding results from Sparse Spacing with Attenuation

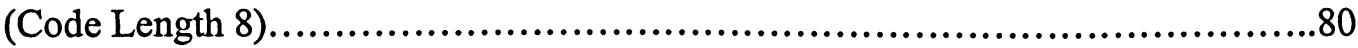

3.4 Backscatter Signal from Sparse Spacing with Plain

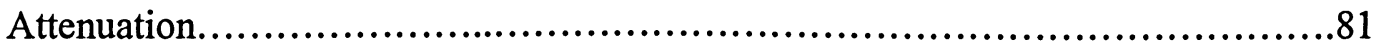

3.5 Encoding results for Sparse Spacing with Plain Attenuation

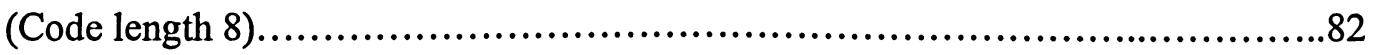

3.6 Backscatter Signal from Sparse Spacing with Frequency Dependent

Attenuation. 
3.7 Encoding results for Sparse Spacing with FD Attenuation

(Code Length 8).

3.8 Backscatter Signal in Dense Spacing without Attenuation

(Code Length 8).

3.9 Encoding results of Dense Spacing without Attenuation

(Code Length 8).

3.10 The STD for Four Code Lengths..........................................88

3.11 Backscatter Signal in Dense Spacing with Plain Attenuation

(Code Length 8). .89

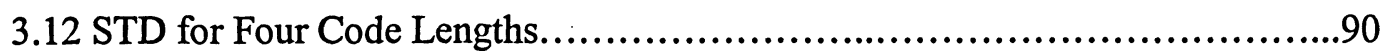

3.13 Backscatter Signal in Dense Spacing with FD Attenuation

(Code Length 8). .91

3.14 STD for Four Code Lengths ..............................................92

3.15 Average STD Ratio for Four Code Lengths..................................93

3.16 The Ratio of STD for Four Code Lengths...................................93

3.17 Signal and Encoding results in Autocorrelation Method........................96

3.18 Signal and Encoding results in Code Reference Method.........................96

3.19 Signal and Encoding results in Code Reference Method........................97

3.20 Phase for Maximal Correlation of Two Processing Methods.....................99

3.21 Signals and their Envelops................................................101

3.22 Encoding results for Phase Shift Effect.....................................103

3.23 Encoding results for Different Code Lengths................................104

3.24 Encoding Gains for Four Code Lengths...................................105

3.25 One Reference from One Cycle Pulse and Envelope...........................107

3.26 Encoding results for Four Code Lengths.....................................108

3.27 Backscatter Signal and Encoding Results and

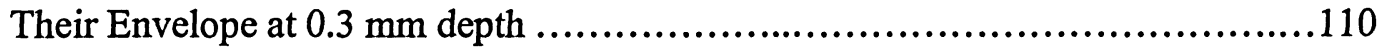

3.28 Backscatter Signal and Encoding Results and

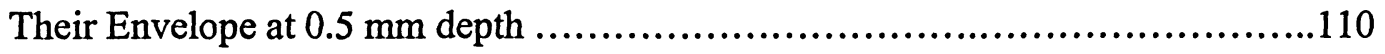

3.29 Backscatter Signal and Encoding results and 
Their Envelope at $0.7 \mathrm{~mm}$ depth ..................................................111

3.30 Reference Signal at Critical Depth..........................................112

3.31 Backscatter Signal and Encoding results for Different Code

Lengths....................................................................113

3.32 The Code Length and Encoding Gain........................................114

3.33 White Noise and Its Encoding results.....................................115

3.34 Backscatter Signals from Bovine Liver.....................................116

3.35 Different Methods (Code Length 2)........................................117

3.36 Effect of Phase Shift ........................................................119

3.37 Envelope Method to Evaluate Signal Similarity ..............................120

A1.1 The Interface of Arbitrary Signal Generator..................................131

A1.2 The Structure of Arbitrary Signal Generator..................................131

A1.3 How to Generate the Encoded Signal........................................135

A1.4 Schematic of High Pass Filter...................................................141

A1.5 Schematic of High Pass Filter................................................141

A1.6 Simulation Results of the Filters..........................................142

A1.7 PCB Layout for Filters............................................... 143

A1.8 Protection Circuit...................................................144

A1.9 Schematic Picture of Expander...........................................145

A1.10 PCB Layout of Expander.............................................145

A1.11 Transmission Line..........................................................146

A1.12 Lab Platform....................................................... 147

A1.13 System Connection Diagram..........................................148

A1.14 Consecutive Pulses..................................................151

A1.15 New Pulser and Its Backscatter Signal..................................151

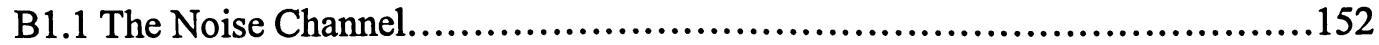

C1.1 Equivalent Circuit of Transducer........................................155

C1.2 Transformer for Impedance Matching.........................................157 


\section{Chapter 1 Introduction}

At the beginning of this chapter, the history of ultrasound and nature of sound are briefly introduced. Then important concepts in ultrasound physics, which are essential for an ultrasound system, are discussed. The concepts include wave fundamentals and the ultrasonic transducer. In the "wave fundamentals" section, the wave equation, acoustic properties of media, reflection and refraction, diffraction and scattering, and intensity are introduced. In the section "ultrasonic transducer", the ultrasonic transducer, equivalent circuit, impedance matching and focusing are discussed. Special emphasis is placed on features significant to medical ultrasound, such as velocity of medium, attenuation, absorption, scattering by biological materials, scattering strength, pulse echo response and single particle model, thermal effect, and cavitation. In the end, the knowledge of the transducer system and coded excitation ultrasound methods are discussed in detail. The components of an ultrasound system and a coded excitation system, two coded excitation methods and their properties are discussed. All of these contents constitute the fundamentals for the rest of the chapters in the thesis. And

a lot of the material has been adapted from the following books: Cobbold's ${ }^{[6]}$ and Wells, ${ }^{[54]}$.

\subsection{Brief History}

Ultrasonics is the study and application of high-frequency sound waves, usually in excess of $20 \mathrm{KHz}$. The roots of ultrasonic technology can be traced back to research on the piezoelectric effect conducted by Pieerre Curie around 1880 . He found that asymmetrical crystals such as quartz and Rochelle salt generate an electric charge when mechanical pressure is applied. Conversely, mechanical vibrations are obtained by applying electrical oscillations to the same crystals. Sonar, the first application for ultrasound, was employed on a large scale by the 
U.S. Navy during the World War II to detect enemy submarines.

The medical application of ultrasound dates back to the early 1950s. The development spans the first crude systems for acquiring single lines of data to today's imaging system. The evolution has passed through several intermediate steps. "A-scope ultrasound systems" were employed by several research groups (Wild 1950; Gordon 1950) to demonstrate that ultrasound could be used for detecting tissue layers, tumors, and heart structures. "B scope ultrasound systems" appeared to sweep a two-dimensional image of tissue structure in 1954 by groups such as Howry and Baum. Later, multi-element transducers (linear arrays and phase arrays) were used in modern scanners (Macovski 1979). "Continuous wave Doppler system" was suggested by Satomura (1957) to use to study the blood flow or probe soft tissue. "Real time imaging systems" were developed by Henry to quickly measure tissue (1967). "Computerized tomography system" created by Greenleaf was to observe the three-dimensional image of tissue structures (1974) [53]|12]|6].

Ultrasound applications in medicine are the most rapidly advanced areas. Ultrasound is used for imaging the human body, as a means to treat various tissue ailments and for sterilizing surgical instruments. Ultrasonic scanning has big advantage over X-ray in that there are apparently no adverse health effects. For this reason, it has come into widely spread use for monitoring the condition of the fetus as it grows in the womb. The increasingly high precision of such monitoring has made it possible to detect defects even at the very early stages of pregnancy. Ultrasonic scanning is also useful for obtaining information about the flow of blood through the carotids. Other important diagnostic applications are the detection of kidney stones, gallstones and tumors.

With further progress in materials (particularly piezoelectric materials for the transducers) in electronics and in computers, improvements in the performance of 
ultrasound system will be accompanied by the reduction in cost. Therefore, the ultrasound technology is now employed in a wide range of applications in industries.

\subsection{Statement of Problem and Organization of Thesis}

The central interest is the high frequency ultrasound system $(30-50 \mathrm{MHz})$. The resolution of an ultrasound system increases with the frequency of the ultrasound. It is a powerful clinical tool that is often used in ophthalmology and dermatology. It has been used in the diagnoses of intra-ocular eye tumours such as choroidal melanoma and conditions such as acute angle glaucoma. It has been recently discovered that it can be used to detect the effects of cancer therapies induced by standard chemotherapeutic agents and radiotherapy. It also can be used to assess organ suitability for transplantation procedure. There are some problems in using high frequencies, one of them is limited penetration depth at these frequencies into tissue (about $1 \mathrm{~cm}$ or less) ${ }^{[21]}$. Therefore, the goal of my thesis is to investigate the potential of using coded excitation for high frequency ultrasound to improve the Signal to Noise Ratio (SNR) from tissue backscatter.

To this end, I perform theoretical simulations to show the expected improvement using an encoding method with Golay Pairs, which included the effects of frequency dependent attenuation, the first such simulations performed. I demonstrate that even when frequency dependent attenuation is included, significant improvements in SNR could be realized. Furthermore, I built a prototype high frequency ultrasound system with coded excitation, to my knowledge to first one ever implemented, to experimentally demonstrate the predicted gains. I further analyzed different decoding reference schemes and found the optimal encoding gain and discussed the results, and some of the issues involved in implementing this clinically. 
The thesis is organized as follows: first, the basics of ultrasound are introduced followed by an introduction to coded excitation. In Chapter 2, which discusses the methods used, I present the theoretical models used for the simulations in the first part and the construction of the experimental prototype in the second part. In Chapter 3, I show the results of the theoretical simulations and then continue to present experimental results using the prototype ultrasound pulse-echo system we developed. Finally, in Chapter 4 I discuss some problems associated with the clinical implementation of the system and potential ways to address them. If you are familiar with physics of ultrasound, you can skip these sections and directly begin with section 1.9 .

\subsection{Nature of Ultrasound}

Sound is the rapid oscillatory motion of molecules. It is produced while applying a time-varying stress to a medium. The propagation of sound waves requires physical elastic media, such as gas, liquid or solid. When the applied stress displaces a particle of the medium is displaced from its equilibrium position, internal forces tend to restore the particle back to its original position. Particles of the media are not propagating away from the medium. They only vibrate back and forth about their equilibrium positions. When many particles vibrate continuously along media, a wave forms. Wave is the disturbance whose position in space changes with time. Mechanical vibrations become vibrating pressure waves, transferring energy to the medium and to objects that the wave contacts by the contact between masses of the medium. As a sound wave propagates in a medium, the mechanical energy moves progressively from particle to particle.

There are three major modes of acoustic wave propagation. They differ in terms of their particle motion in relation to the sound wave propagation direction. The 
simplest form is that a plane longitudinal (compressional) wave propagates in a homogeneous isotropic medium of semi-infinite extent. The particle motion is parallel to the wave propagation direction. The second simplest form is that in the solid medium the particle movement is at right angles to the direction of propagation. This wave is called a transverse (shear) wave. If the plane boundary of a semi-infinite solid separates the solid from a rarefied medium (e.g. gas), then wave propagation can occur close to the boundary through a coupling of longitudinal and transverse waves at the interface. Surface waves have an elliptical particle motion. These waves travel across the surface of the material with the major axis of the ellipse perpendicular to wave propagation direction. Rayleigh first described such waves in 1885 . Therefore, they are often referred to as Rayleigh waves. Longitudinal waves may present in any elastic or compressible media such as solid, liquid and gas. However, the other forms of wave modes rarely propagate in liquid and gases.

Frequency is defined as the number of cycles per second of a harmonic vibrating disturbance. As shown in Figure 1.1, the acoustic spectrum breaks down sound into three ranges of frequencies: subsonic range, audible range, and ultrasonic range. Ultrasonic wave refers to the human-inaudible sound wave whose frequency range is above $20 \mathrm{KHz}$, the upper frequency response limit of the human ear. The ultrasonic range is then further divided into three subsections. Ultrasound behaves in a similar manner to audible sound except that it has a much shorter wavelength. Generally the ultrasound frequency range employed in medicine is $100 \mathrm{KHz}$ to $10 \mathrm{MHz}$. Now many research articles are published on higher frequency range (from $20 \mathrm{MHz}$ to $100 \mathrm{MHz}$ ) that offer better resolution images. Generally, the high frequency ultrasound systems are used for medical imaging. The lower frequencies, $1 \mathrm{MHz}$ or less, have longer wavelengths and greater power at a given input energy. For signals at low frequency last longer time than those at high frequency, thus producing greater disruption of the medium and are used in therapeutic applications. In recent years, higher resolution, 
real-time and color images and 3-D imaging are the trends for ultrasound image systems.

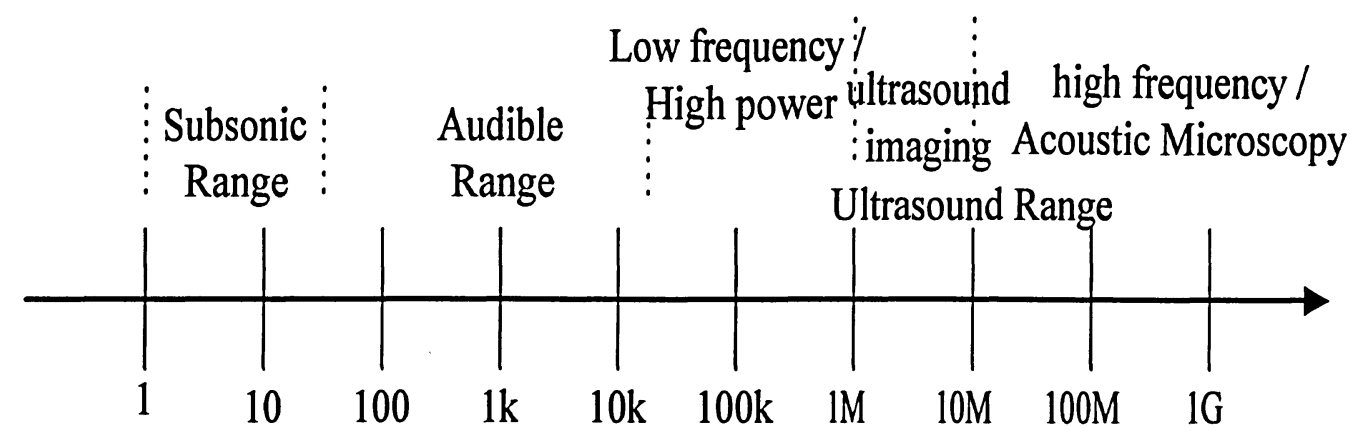

Figure 1.1 Acoustic Spectrum (in Hz)

\subsection{Wave Fundamentals}

Wave fundamentals include wave equation, acoustic properties of a media, reflection and refraction, diffraction and scattering and intensity. All information will be helpful for us to understand the phenomena in our experiments.

\subsubsection{The Wave Equation}

As equation (1.1), the wave equation shows us how the wave travels along the media.

$$
u=u_{0} \sin ((2 \pi / \lambda)(c t-z))=u_{0} \exp (j(\omega t-k z))
$$

The quantity $2 \pi / \lambda$ is called the wave number; $\mathrm{c}$ is the velocity of wave travels along the $\mathrm{z}$ direction; the $\mathrm{u}_{0}$ is the amplitude of the wave. Longitudinal waves travel in a medium at a velocity $\mathrm{c}=(\mathrm{K} / \mathrm{\rho}){ }^{1 / 2}, \mathrm{~K}$ is the bulk modulus of the liquid transverse and density $\rho$. Waves travel along a string at a velocity $c=(F / \eta)^{1 / 2}, F$ is tension on the string and $\eta$ is the linear mass density of the string. 
In medical ultrasound systems, generally soft tissues are the media for ultrasound propagation. The force on particles can be expressed in equation 1.2.

$$
p=K \frac{\partial u}{\partial z}
$$

From $c=(K / \rho){ }^{1 / 2}$, we get $K=\rho c^{2}$

$$
p=\rho c^{2} \frac{\partial u}{\partial z}
$$

And from equation (1.1), $\frac{\partial u}{\partial z}=-\frac{1}{c} v ; v$ is the particle velocity

$$
p=\rho c v
$$

\subsubsection{The Acoustic Properties of Media}

Impedance is an important concept that characterizes wave propagation in the presence of boundaries between media with various acoustical properties. As applied in mechanics, the relationship between pressure, the velocity, and the quantity $\rho c$ is analogous to that which exists in electricity between voltage and current and impedance (see Ohm's law). The ratio of force amplitude to velocity amplitude is referred to as impedance.

$$
Z=p / v
$$

Webster introduced impedance into acoustics in 1914. The specific acoustic impedance of an acoustic field is defined as the ratio of acoustic pressure to the associated particle speed on a medium. The simplest situation occurs with the propagation of a plane wave in an inviscid fluid medium. By substituting the pressure-velocity relation (1.4) given by into (1.5) we obtain

$$
Z=Z_{0}=\rho c
$$

where $Z_{o}$ is known as the characteristic (acoustic) impedance of the medium, $\rho$ is the mass density of the medium, $\mathrm{c}$ is the propagation speed of the sound wave. 
Table 1.1 Characteristic Acoustic Impedances of Selected Media ${ }^{[6][53]}$

\begin{tabular}{|l|c|l|c|}
\hline \multicolumn{1}{|c|}{ Medium } & $Z_{o}$, MRayl & \multicolumn{1}{|c|}{ Medium } & $Z_{o}$, MRayl \\
\hline Aluminum & 17. & Dry Air & 0.0004 \\
\hline Steel (mild) & 44 & Water, & 1.5 \\
\hline Brass & 38 & Blood, Whole Human & 1.67 \\
\hline Mercury & 20 & Bone, Fresh Bovine Phalanx & 7.9 \\
\hline
\end{tabular}

The characteristic acoustic impedance of a medium is defined as the product of the mass density $\rho$ and the speed of sound $\mathrm{c}$ of the medium. It has the S.I. unit of Rayl. The specific acoustic impedance for a plane wave equals the characteristic acoustic impedance of a medium. In Table 1.1 the impedances of various media are listed. We notice the impedance of air is so small that we need some water or other materials to match the impedance to the tissue be diagnosed. Otherwise all the energy is lost in reflection.

\subsubsection{Reflection and Refraction ${ }^{[12][6] \mid 53]}$}

When an acoustic wave traveling in one medium encounters the boundary of a second medium, reflected and transmitted waves are generated. The following conditions must be satisfied at the interface: (i) the pressure is continuous; (ii) the particle velocity component normal to the interface is continuous. So pressure reflectivity and pressure transitivity of the interface at normal incidence are in equation (1.7) and (1.8)

$$
\begin{aligned}
& \mathrm{R}=\frac{p_{r}}{p_{i}}=\frac{Z_{2}-Z_{1}}{Z_{2}+Z_{1}} \\
& \mathrm{~T}=\frac{p_{t}}{p_{i}}=\frac{2 Z_{2}}{Z_{2}+Z_{1}}
\end{aligned}
$$


$\mathrm{R}$ is reflectivity and $\mathrm{T}$ is transitivity; $p_{i}$ incident pressure; $p_{r}$ reflection pressure; $p_{t}$ transmission pressure; $\mathrm{Z} 2$ and $\mathrm{Z} 1$ are impedances for two media.

\subsubsection{Diffraction and Scattering ${ }^{[12][6][53]}$}

Two factors may cause the ultrasonic wave to spread or diverge in its propagation path in the medium: diffraction at the source and scattering by obstacles. Diffraction means the bending of waves around small obstacles and the spreading out of waves beyond small apertures. It always occurs at the edge of a finite source aperture. Since an aperture has to be finite, any practical sound wave that is radiated by a source will eventually diffract. Scattering can be defined as the change of amplitude, frequency, phase velocity, or direction of propagation of a wave as a result of spatial or temporal non-uniformities of the medium. The inhomogeneities arise due to variations in compressibility or acoustic impedance differences. As the scattering of the sound wave occurs, the secondary sound spreads out in different directions.

Scattering leads to spatial redistribution of acoustic energy, which in turn changes signal amplitude. The behavior of scattering depends on factors such as absorption, acoustic impedance mismatch, spatial distribution and scale of inhomogeneities, shape of scatterer, number of scatterers per unit volume and medium motion, etc. Absorption of ultrasound is the transformation of ultrasonic energy to heat by frictional forces. The energy loss mechanisms in absorption attenuation are both frictional force and relaxation in nature. In general, the degree of absorption is directly related to the frequency. Acoustic impedance mismatch occurs whenever there is a magnitude difference of sound speed, mass density, or compressibility between the scatter inhomogeneity and its surrounding medium. The greater the mismatch, the more significant is the inhomogeneity and the stronger is the 
scattering produced. The scale of inhomogeneities, including the size of scatters and the interspacing between them, is often used to categorize different scattering phenomena. Different sizes of scatterers follow different rules.

\subsubsection{Intensity ${ }^{[17]}$}

The acoustic intensity of a wave is the average flow of energy through a unit area normal to the propagation direction per unit time. The intensity is the average of the rate of work done per unit area by one element of fluid on an adjacent element

$$
I=p v=\frac{1}{T} \int p v d t
$$

And

$$
\begin{aligned}
& v=p / Z \\
& p=p_{0} \exp (j(\omega t-k z))
\end{aligned}
$$

So that

$$
I=\frac{1}{T} \int_{0}^{T} \frac{p_{0}^{2}}{Z} \exp (2 j(\omega t-k z)) d t=\frac{p_{0}^{2}}{2 Z}
$$

From this equation, the intensity is the square of pressure $p$.

\subsection{Ultrasonic Transducers}

The transducer is the heart of any medical ultrasound system. It converts the mechanical energy into electrical energy and vice versa. There has been a steady progression and refinement in transducer design over the past three decades. In the early days, people used single element, unfocused transducer. Based on the work of $\mathrm{O}^{\prime} \mathrm{Neil}^{[35]}$, the advantage of using gently focused transducers was apparent. The development of phased annular arrays provided a means of strongly focusing the received beam while maintaining a good depth of field. Around 1968, Somer 
extended the phased array concept with a linear arrangement of transducers whose beam could be focused and steered across the body by the application of suitable delays to each element ${ }^{[15]}$. Afterwards, the real time pulse-echo systems based on simple linear arrays began to proliferate.

\subsubsection{Parameters and Materials}

There are several parameters used to describe the piezoelectric properties. Transmitting constant is the strain produced in a piezoelectric material by application of a unit electric field. Receiving constant is the electric field produced under open-circuit conditions per-unit applied stress. Coupling Factor describes the relation of output mechanical or electrical energy to the stored energy in a transducer. For a transmitter, a large transmitting constant is desirable; for a receiver, a large receiving constant is expected. If a transducer operates as a transmitter and a receiver, it is desirable for both the transmitting constant and the receiving constant to be large.

Several techniques, such as magnetostriction, electrostriection and thermally excited shock waves, have been used to generate ultrasound. The most practical devices for medical purpose are based on the piezoelectric effect. Barium titanate, lead zirconate titanate (PZT) and Polyvinlidene difluoride (PVDF) possess strong piezoelectric properties. As a special class of piezoelectric ceramics, PZT is a successful material for medical transducers thanks to its strong piezoelectric properties and high Curie temperature. Furthermore, a lot of mixture can be added to PZT to change the composition a little, resulting in a wide range of materials optimized for different applications. But inherently high acoustical impedance of PZT greatly limits the application in construction of efficient, wideband transducers for medical scanning. Polyvinlidene difluoride (PVDF) is a type of plastic that has a very strong piezoelectric property and relative low impedance. 
The drawback of PVDF is its lower coupling factors and high dielectric losses. When we design an ultrasound system, the information on transducer equivalent circuit and impedance matching is important. It is discussed in Appendix C.

\subsubsection{Focusing Field}

An unfocused transducer emits an ultrasonic beam which spreads radially because of diffraction. The beam intensity falls off and the beam diameter is too large to obtain good lateral resolution when probing an object in the far field. Therefore, a focused acoustic beam is often used, as in optics, to obtain good lateral resolution and high acoustic intensity at a point of interest. The focused ultrasound pulse waves in a focal region are used so that the response of the microstructure in this focal region to the incident ultrasound wave could be probed with high sensitivity.

Focused ultrasound pulse waves can be generated by a single element transducer with concave surface such as spherical or cylindrical surface which functions similarly for a focusing optical lens. There are essentially three approaches to focus an ultrasound beam: shaping the actual transducer vibrating element, attaching a concave lens to the transducer face, and inserting a biconvex lens into the ultrasound energy path which is similar to focusing the light from the sun using a magnifying glass.

The sound field of a transducer is a beam within which sound intensity varies. An unfocused beam can be divided into the near field and the far field. The near field is the region directly in front of the transducer where the echo amplitude goes through a series of maxima and minima and ends at the last axial maximum, at distance $N=D^{2} / 4 \lambda$ from the transducer, where $D$ is the diameter of the transducer surface. The far field is the region at distances greater than N. Attenuation is effectively a gradual loss of energy. The ultrasound beam attenuates as it progress 
through a medium. Attenuation in the near field is associated with edge diffraction, absorption and scattering. In far field, beam spread joins the three factors to attenuate sound intensity of the beam.

In Figure 1.2, A spherical focusing transducer has the following important characteristics: diameter $\mathrm{D}$, focal length $\mathrm{F}_{\mathrm{L}}$ which is the distance between the center point on transducer surface and the point of maximum echo from a point target, focal depth $D_{z}$ which is the pulse echo beam axial distance between two points whose echo amplitudes are $-6 \mathrm{~dB}$ relative to the focal point amplitude, beam diameter $\left(D_{x}\right)$ which is the $-6 \mathrm{~dB}$ pulse echo beam lateral diameter at $F_{L}$ the numerical aperture of a transducer is defined as the ratio of its focal length to its diameter $\left(f_{\#}=F_{L} / D\right)$. The wave form of a transducer has the following parameters: center frequency

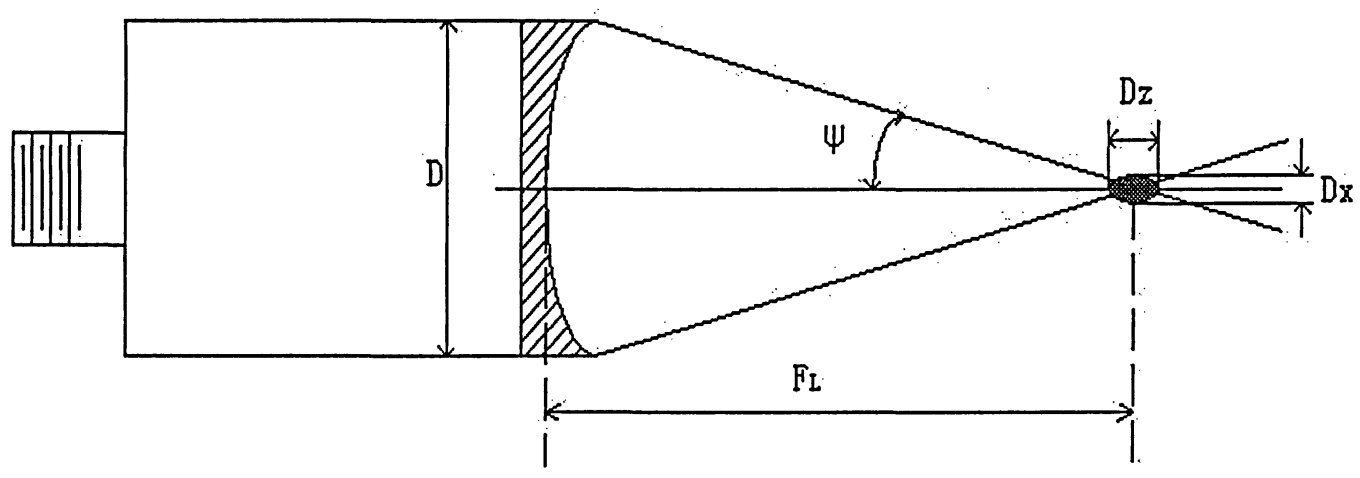

Figure 1.2 Focusing Transducer ${ }^{[15] \mid 54]}$

$\left(f_{0}\right),-3 d B$ frequency bandwidth $(\Delta \mathrm{f}(-3 \mathrm{~dB}))$, pulse duration which is the wave duration at the $-20 \mathrm{~dB}$ level or $10 \%$ amplitude per second. A transducer is often described by its center frequency $f_{0}$, diameter $D$ and numerical aperture $f_{\# \text {. }}$.

The focal volume is roughly ellipsoidal, the intensity being reduced by $3 \mathrm{~dB}$ from the maximum value in $x-y$ plane where the diameter is given by

$$
D_{y}=D_{x}=\frac{K_{t} F_{L} \lambda}{2 D}
$$


where $\mathrm{K}_{t}$ is a dimensionless factor the value of which is dependent upon $\psi, \psi<50^{\circ}$, $\mathrm{K}_{\mathrm{t}}$ is taken to be equal to 1.0. The length of the focal volume in the central z-axis is given by

$$
D_{z}=K_{a} D_{x}
$$

where $K_{a}$ is a dimensionless factor, generally take it to be equal $15 \times(1-0.01 \psi), \psi$ being expressed in degree. The intensity "gain" of a focusing system is defined as

$$
G=\frac{I_{F}}{I_{0}}
$$

$I_{F}$ is the intensity at the focus, the $I_{0}$ is the intensity at the surface of transducer.

\subsection{Velocity, Attenuation and Absorption}

\subsubsection{Velocity of Sound in a Medium}

The speed of sound and the mass density are essential acoustic properties of a medium. The propagation speed of a longitudinal sound wave in a fluid medium (c) is $(K / \rho)^{1 / 2}$. Generally sound propagates faster in solids than in liquids, and faster in liquids than in gases. We employ a single velocity for one kind medium. This is true for most of the cases, but strictly the velocity will have a little dispersion in one kind of medium with many factors such as temperature, frequency and absorption characteristics.

In Figure 1.3, the accurate measurements on the propagation speed in pure water

over the temperature range from 0 to $90^{\circ} \mathrm{C}$ are shown. For the temperature (10 to $40^{\circ} \mathrm{C}$ ), the range of propagation speed is 1450 to 1530 . In our simulation we use $1500 \mathrm{~m} / \mathrm{s}$, corresponding to $27^{\circ} \mathrm{C}$ (room temperature). 


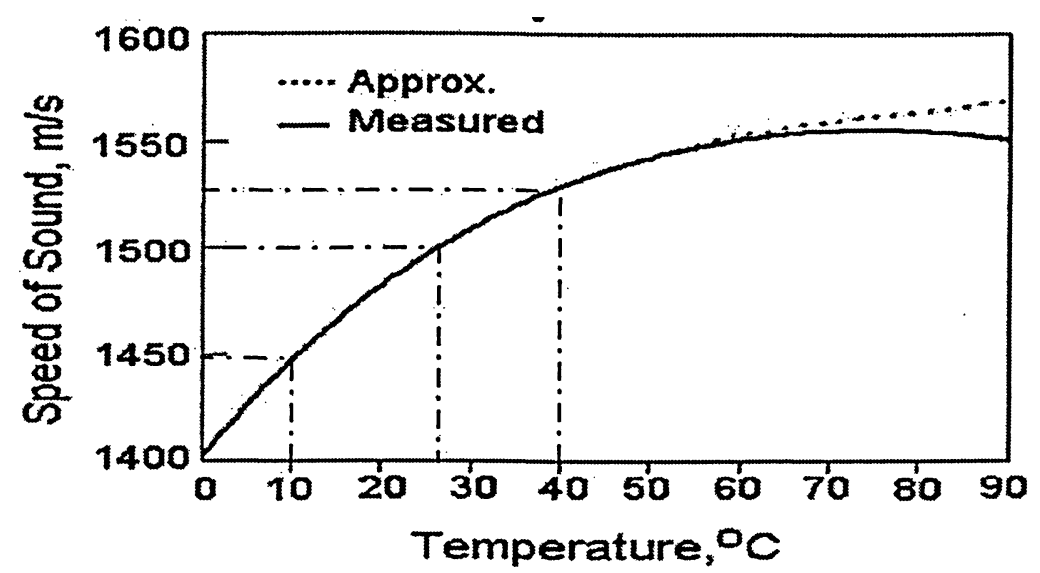

Figure 1.3 The Speed in Pure Water at $5 \mathrm{MHz}$ with Temperature 0-90 ${ }^{[6]}$

Since the speed of water is always used as the reference to measure the speed of the biological medium, the accurate measure of the speed of pure water is critical. Biological specimens are constantly far from being ideal in terms of their shape and achieving a good accuracy requires considerable care. One of the convenient methods is the substitution method based on observing the change in transit time of short ultrasound pulse when the specimen is removed from the propagation path and is replaced by a fluid whose speed is known.

\subsubsection{Attenuation of Biological Medium}

\subsubsection{Attenuation}

When a wave travels through a real medium, its intensity is reduced as a function of distance. In practical, several factors contribute to this attenuation. These factors include: scattering by non-specular reflection, so that a propagation of the energy no longer moves in the original direction of propagation; and absorption, by which ultrasonic energy is converted into heat due to viscous loss.

Assume the amplitude of a plane wave propagates in the $\mathrm{z}$-direction, so that the wave can be expressed in the form:

$$
A_{z}=A_{0} e^{-\mu z}
$$


Where $A_{0}$ is the peak value at $\mathrm{z}=0$ of a wave variable such as particle velocity or the amplitude of the particle movement; $A_{z}$ is the peak value at $\mathrm{z}$ of the same variable; $\mu$ is the amplitude attenuation coefficient; $z$ is the location in $z$-direction. It can be written in terms or either the intensity or pressure and is generally expressed in $\mathrm{dB} / \mathrm{cm}$ (decibels $/ \mathrm{cm})$.

$$
\mu_{d B}=\frac{1}{z} \times 10 \log \left(\frac{I_{0}}{I_{z}}\right)
$$

The presence of attenuation results in a phase difference between the pressure and the particle velocity, causing the specific acoustic impedance to contain both real and imaginary parts. Nonetheless, for attenuation coefficients that are not too large the pressure amplitude $\mathrm{p}^{2} \propto \mathrm{I}$, so that

$$
\mu_{d B}=\frac{1}{z} \times 20 \log \left(\frac{p_{0}}{p_{z}}\right)
$$

In the literature, $\mu$ is often quoted in units of nepers per centimeter, so that:

$$
\mu_{N p}=-\frac{1}{z} \ln \left(\frac{A_{0}}{A_{z}}\right)
$$

Different tissues have different attenuation coefficients; the values for different tissues are listed in Table 1.2.

Table 1.2 Approximate Attenuation Values for Human Tissue ${ }^{[6][54 \mid[12]}$

\begin{tabular}{|l|l|}
\hline Tissue & Attenuation $\mathrm{dB} /[\mathrm{MHz} . \mathrm{cm}]$ \\
\hline Liver & $0.6-0.9$ \\
\hline Kidney & $0.8-1.0$ \\
\hline Spleen & $0.5-1.0$ \\
\hline Fat & $1.0-2.0$ \\
\hline Blood & $0.17-0.24$ \\
\hline Plasma & 0.01 \\
\hline Bone & $16.0-23.0$ \\
\hline
\end{tabular}


For an attenuation of $0.7 \mathrm{~dB} /[\mathrm{MHz} . \mathrm{cm}]$, the sound be attenuated $2 \times 1 \times 40 \times 0.7=$ $56 \mathrm{~dB}$ for a received signal from a $40 \mathrm{MHz}$ transducer investigating tissue $1 \mathrm{~cm}$ from the transducer, as the sound moves into the tissue twice.

\subsubsection{Frequency Dependent Attenuation}

The tissue attenuation is dependent on frequency with an increasing attenuation for increasing frequency. Since attenuation is a combination of the effects of absorption and scattering, its frequency dependence combines the frequency dependent attenuation of both factors. For pure fluids there will be no scattering and as a result the attenuation and absorption characteristics are identical. For tissues, absorption accounts for 75 to $95 \%$ of the total attenuation ${ }^{[37]}$.

Several experimental methods have been developed to determine the attenuation versus frequency characteristic of biological specimens. For most biological tissues the attenuation can be characterized by $\mu(f)=\mu_{0} f^{n}$, where $\mu_{0}$ denotes a temperature dependent constant with units that can be expressed in $\mathrm{Np} /\left(\mathrm{cm} \cdot \mathrm{MHz}^{2}\right)$, $f$ is the frequency and expressed in MHz. $n$ is generally around 1, but may lie in the range $1 \leq n<2$ (Figure 1.4). For pure fluids there will be no of scattering so all attenuation come from absorption. As illustrated in Figure 1.4, $(\mathrm{n}=2$ for water and $\mathrm{n}=0.9$ to 2.1 for bone), although the attenuation is generally much higher than soft tissue, it also exhibits a power law dependence over a more limited frequency range. A lot of methods have been made to use of the attenuation versus frequency characteristics as a means of identifying abnormal tissue. ${ }^{[6]}$ 


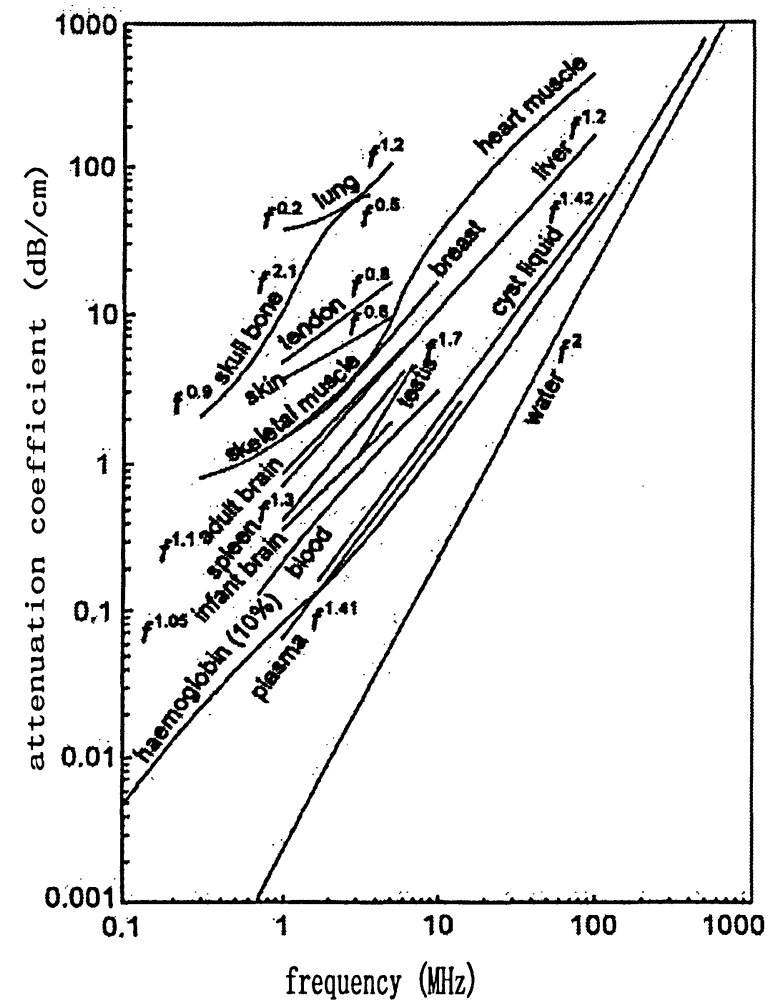

Figure 1.4 Tissues' Frequency Dependent Attenuation Coefficient ${ }^{[6]}$

Due to the very complex composition of tissue, it has not been possible to derive a simple model that can describe the frequency dependence of the attenuation and most literature resorts to a simple phenomenological model. Frequency dependence attenuation into a frequency domain method of solution is straightforward. Expressed in the frequency domain, the amplitude attenuation transfer function for a plane wave propagating through tissue is:

$$
H(f, z)=\exp \left(-\left(\mu_{0}+\mu_{1} f\right) z\right)
$$

where $\mathrm{z}$ is the depth in tissue, $\mathrm{f}$ is frequency, $\mu_{0}$ is frequency independent attenuation coefficient, $\mu_{1}$ is frequency dependent term. So the wave can be expressed in frequency domain:

$$
p(f)=p_{0}(f, z) * H(f, z)=p_{0}(f, z) * e^{-\left(\mu_{0}+\mu_{1} \delta\right) z}
$$

This is an alternative approach to get frequency dependent attenuation signals. First Fourier transform the waveform, multiply by the transfer function and then perform an inverse Fourier transform to obtain the attenuated waveform. These 
approaches will be used in Section 2.3.2.2 to model attenuation.

\subsubsection{Absorption of Medium}

Absorption accounts for 75 to $95 \%$ of the total attenuation ${ }^{[37]}$. The classical mechanism proposes that the absorption of ultrasound in fluid is due to the frictional force that acts to oppose the periodic motion of the particles in the medium. In addition to the classical theory of absorption due to viscosity, several other mechanisms may contribute to attenuation depending upon the properties of the propagating medium and the frequency of the wave, including relaxation processes, relative motion, bubble mechanisms and hysteresis.

Relaxation processes contributes to the ultrasonic absorption in biological materials. Energy in a system can exist in various forms, such as molecular vibration energy, lattice vibration energy. Energy increases in one or more of these forms when ultrasound wave passes through a medium. All the different forms in which the energy can be stored are coupled in various ways. Consider a small element of volume in a medium supporting an ultrasonic wave. A temperature rise causes an increase in the energy that the wave carries (due to the speed of sound increase with temperature). If none of this energy transformed into another form, the increased energy for temperature reason will return back to the vibration energy of the wave during the de-compressive part of the cycle, and no absorption will occur. In reality, however, this excess transmitted energy will change into another form during the compression cycle. During decompression, some of energy returns out of phase with the traveling wave, which causes absorption.

Relative motion is produced between biological cells and their surrounding medium by the ultrasonic field. It consumes some energy of the wave. The presence of gas bubble in the propagating medium will have a great influence on the biological materials. Almost all the energy will be sent back due to the 
mismatch impedance. Hysteresis will occur, when the frequencies greater than the viscous relaxation frequencies. An excess energy will be consumed in a very viscous media.

Ultrasound diagnosis is less harmful than X-ray, but there are some side effects on biological tissue. Biological effects are discussed in Appendix D.

\subsection{Scattering by Biological Materials ${ }^{[8]}$}

\subsubsection{Introduction}

As we discussed in Section 1.4.4, scattering causes spatial redistribution of acoustic energy. Scattering behavior depends on several factors such as absorption, acoustic impedance mismatch, spatial distribution and scale of inhomogeneities, shape of scatterers, number of scatterers per unit volume and medium motion. If the scattered signals can be captured, it will contain a lot of information on the tissues, shape and angle of the boundaries between them, the structure and properties of tissues, and so on. In Figure 1.5, we can see the foot, mouth, spine and head of the fetus. There is a clear boundary between the fetus and the surrounding amniotic fluid. The skull boundary is not visible in the image, because there is the angle between the beam and the boundary and the sound back off in another direction. Still we can see the head of fetus due to the backscatter signal of ultrasound wave. Small changes in density, compressibility, and absorption give rise to a scattered wave radiating in all directions. And the backscatter signal is received by the transducer and displayed on the screen. 


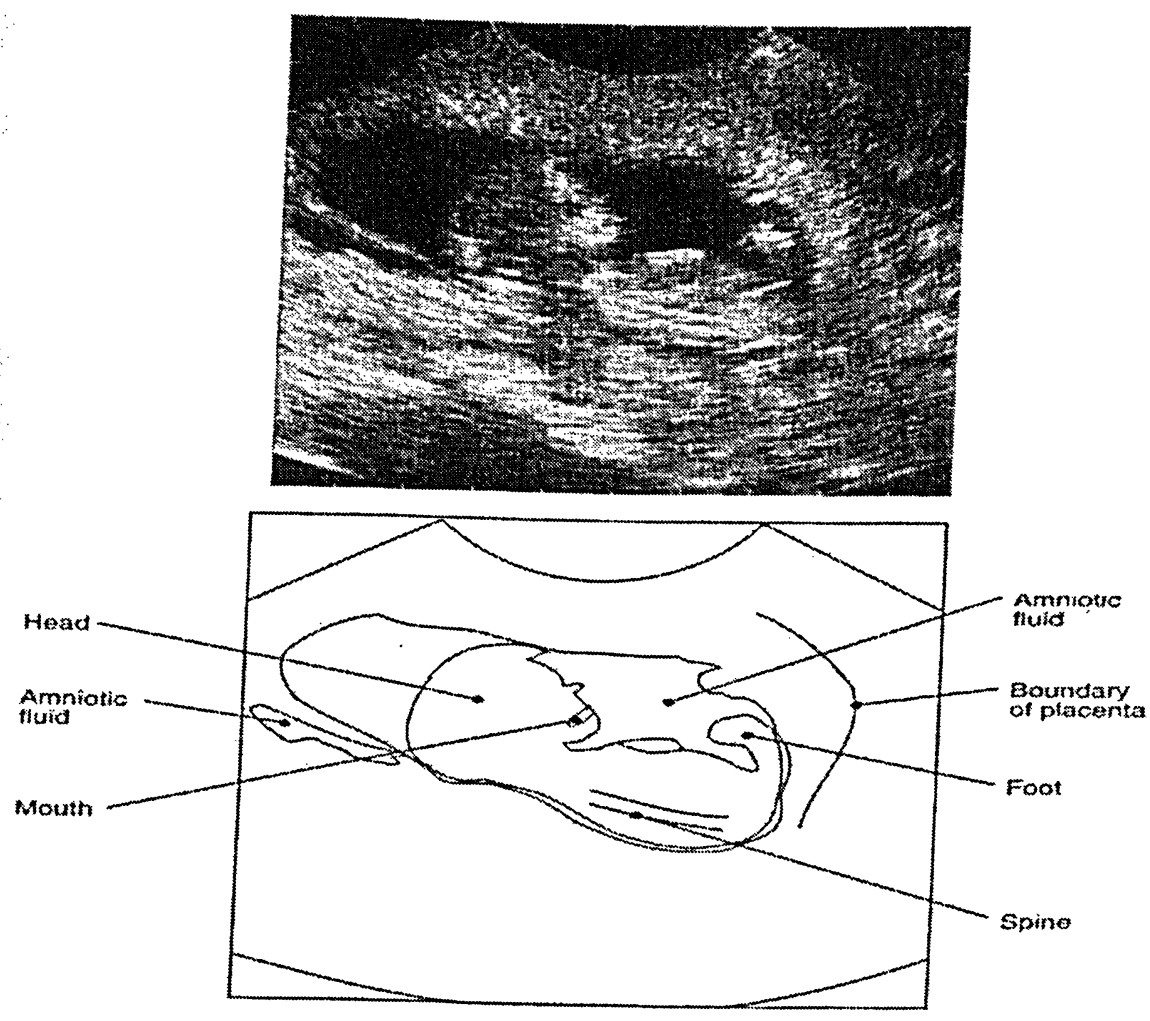

Figure 1.5 Ultrasound Images of $13^{\text {th }}$ Week Fetus ${ }^{[17]}$

As the scattered wave is formed from numerous contributors, it is appropriate to characterize it in statistical terms. The amplitude distribution can be characterized in a Gaussian distribution and fully characterized by its mean and variance. Generally the envelope of backscatter signal is considered more valuable information than backscatter signal itself.

Since the backscattered signal depends on the interference of waves from numerous small tissue structures, it is the deviations within the tissue and the composition of the tissue that determine the strength of the returned signal. The magnitude of the returned signal is described in terms of the power of the scattered signal. Since the structures reradiate wave in all directions and the scattering structures might be ordered in some direction, the returned power will be dependent on the relative position between the ultrasound emitter and receiver. 


\subsubsection{Scattering Strength}

Typically, only one transducer is used for transmission and reception in medical ultrasound. If only the backscattered signal is considered, the signal power generated by a single scatterer can be characterized by the scattering cross-section. If the scatterer is small so that the incident field has a uniform intensity, $\mathrm{I}_{\mathrm{i}}$, then the scatter power is

$$
P_{s}=I_{i} s_{s}
$$

where $\mathrm{s}_{\mathrm{s}}$ denotes the scattering cross section in square meters. The scattering cross section is a measure of the scattering magnitude. It is the equivalent area of the scatterer that intersects a beam of intensity $I_{i}$ and reflects the power Ps. The backscattering cross section depends on the material and indicates the scattering strength of the material.

Table 1.3 Contributions of Scattering to the Attenuation Coefficient ${ }^{[6]}$

\begin{tabular}{|l|c|c|c|c|}
\hline \multicolumn{1}{|c|}{ Medium } & $2 \alpha_{s}, \mathrm{~cm}^{-1}$ & $2 \alpha, \mathrm{cm}^{-1}$ & $\alpha_{s} / \alpha$ & Freq. \\
\hline Fresh Human Liver & 0.09 & 0.72 & $12 \%$ & $4 \mathrm{MHz}$ \\
\hline Fresh Human Liver & 0.32 & 1.4 & $23 \%$ & $7 \mathrm{MHz}$ \\
\hline Fresh Skeletal Muscle & 0.16 & 0.94 & $17 \%$ & $4 \mathrm{MHz}$ \\
\hline Fresh Skeletal Muscle & 0.32 & 1.8 & $18 \%$ & $7 \mathrm{MHz}$ \\
\hline Human Blood, Hct $=40 \%$ & $0.28 \times 10^{-3}$ & 0.17 & $0.1 \%$ & $4 \mathrm{MHz}$ \\
\hline Human Blood, Hct $=40 \%$ & $1.8 \times 10^{-3}$ & 0.37 & $0.5 \%$ & $7 \mathrm{MHz}$ \\
\hline
\end{tabular}

Another useful measure of scattering is the backscattering coefficient which characterizes scattering from a volume of scatterers. It is defined as the average power received from a volume of scatterers per sterradian, the unit is $1 /[\mathrm{cm} \cdot \mathrm{sr}]$. As shown in Table 1.3, the backscatter coefficients correlate with frequency positively. At the same time, the scattering takes more part in absorbing the energy, which 
means it is possible that strong backscatter signal can be obtained in high frequency. We notice that the attenuation coefficients also increase as frequencies increase. The penetration depth of high frequency ultrasound will be shallower than that of the lower frequency signal.

That is one of most important reasons why we are interested in backscatter signals in high frequency range. The backscatter signal takes more part of total attenuation energy with frequency. During the experiments we do find that the backscatter signal is stronger with higher frequency. So we hope these stronger high frequency backscatter signals can help us complete some new research. For example, this method might be used to classify different structures of tissue that are difficult to be classified by low frequency ultrasound signals.

\subsubsection{Pulse Echo Response}

In this section, some basic and simple models for ultrasound system will be provided as the basis of our simulation on the binary coded excitation method. For the effect of binary coded excitation is our aim, all the other factors such as backscattering model, attenuation model, coded methods and experimental system are chosen the simplest ones.

The simple ultrasound pulse echo system, such as that used in early diagnostic systems, is the system we developed and tested. 


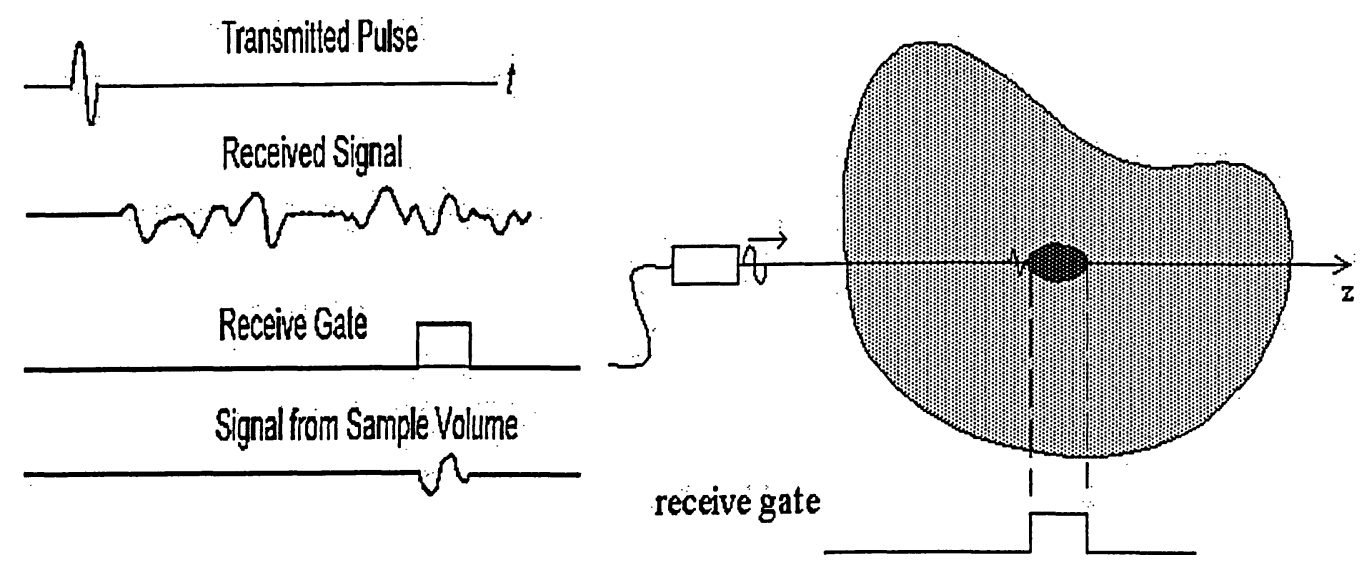

Figure 1.6 Simplified Representation of the Echo Response

As in Figure 1.6, a pulsed pressure is generated by a piezoelectric transducer and emitted into the tissue. The pressure propagates through the tissue and is partly reflected and scattered from changes in density and propagation velocity in the tissue. The backscatter signal is received by the same transducer crystal and amplified by the amplifier. The height of received signal indicates the strength of the signal. The horizontal axis represents time and is equivalent to the depth in the tissue. When assuming the speed is known, the relation of time and the depth of the tissue can be decided. If we send a standard pulse, a time gate can be used to filter the impulse response to a special point of scatter; this is very useful to judge the quality of the backscatter signals of binary coded excitation method.

In our experimental results section, the backscatter signals, when a one-cycle sine pulse is used as an excitated input into transducer, are always sampled and used as an evaluation reference for the results from binary coded excitation method. This backscatter signal from the one-cycle sine pulse input is called one cycle excitation backscatter signal or one cycle excitation.

\subsubsection{Single Particle Model}

As we have just started exploring binary coded excitation methods for high 
frequency ultrasound we used a simple model to simulate the backscatter signal from tissue. Because the complex structure of most biological media, substantial approximations are required to be as tractable scattering models. For soft biological media one such model is based on the assumption that the media consists of a distribution of discrete scatterers imbedded in a background whose acoustic properties are constant. In this particle approach, the scatterers are assumed to be discrete entities with varying geometry and differing acoustic properties, and to have a certain spatial distribution. This is shown in Figure 1.7.

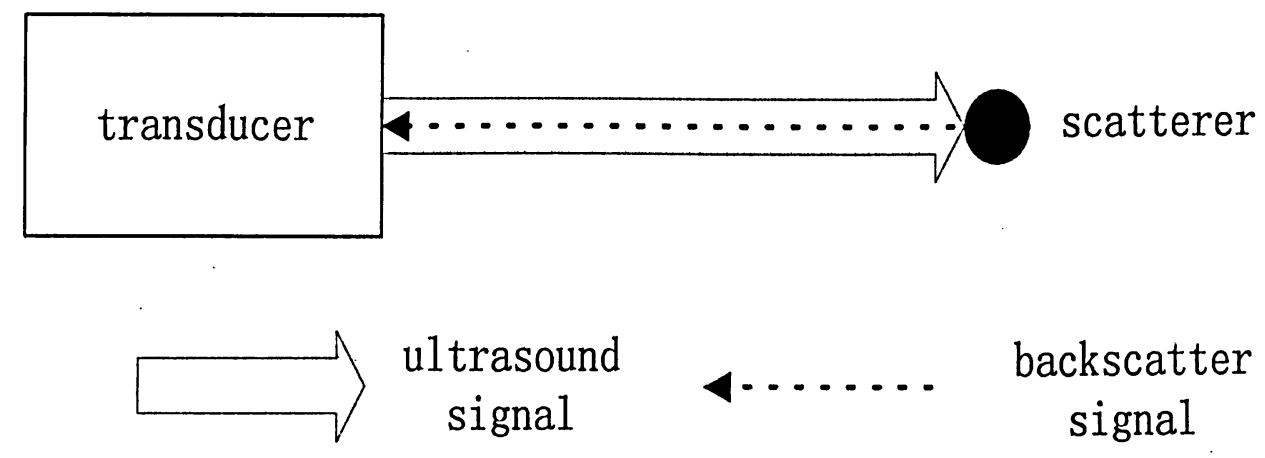

Figure 1.7 Single Particle Model of Scattering

$$
e_{o}(t)=e_{i}(t) * w_{t}^{\delta}(t) * h_{s}(t) * w_{r}^{\delta}(t)
$$

$w_{t}^{\delta}(t)$ and $w_{r}^{\delta}(t)$ are the electromechanical responses of the transmitter and receiver transducers and $h_{s}(\mathrm{t})$ is the pressure impulse response of the scatterer. $e_{o}(t)$ is backscatter signal and $e_{i}(t)$ is the signal sent out from transducer.

\subsubsection{One Dimensional Scattering}

We extend the single particle model to a one-dimensional scattering model. As Figure 1.8, one-dimensional scattering model is good to simulate one line of data acquisition system. The model is simple and effective and is explained in detail in 
Chapter 2. The experimental prototype will make us focus on the coded excitation itself. Now the basic idea of the model will be discussed. For soft biological media, we can think the media consists of a distribution of small volume of scatterers. The small volume of scatterers can be regarded as points. As Figure 1.8(a), when only one line of backscatterers is considered, a distribution of a series of points forms the tissue.

All of ultrasound components are assumed as linear systems. As Figure 1.8 (b) demonstrates, all the points are divided into separate points. For each point, the backscatter signals will be calculated following the single particle model as in Section 1.7.4. When backscatter signals for each point are known separately and linear system properties are used at the same time, the superposition of backscatter signal for each point forms the total backscatter signal for all the points of line.

$$
\begin{gathered}
e_{o 1}(t)=e_{i}(t) * w_{t}^{\delta}(t) * h_{s 1}(t) * w_{r}^{\delta}(t) \\
e_{o N}(t)=e_{i}(t) * w_{t}^{\delta}(t) * h_{s N}(t) * w_{r}^{\delta}(t) \\
e_{o}(t)=e_{o 1}(t)+e_{o 2}(t)+\cdots+e_{o N}(t) \\
=e_{i}(t) * w_{t}^{\delta}(t) *\left[h_{s 1}(t)+h_{s 2}(t)+\cdots+h_{s N}(t)\right] * w_{r}^{\delta}(t)
\end{gathered}
$$

where impulse response of each scatterer is given by

$$
h_{s N}(t)=s_{N}(t) * \delta\left(t-z_{N} / c_{o}\right)
$$

$S_{N}(t)$ is the backscatter strength; $z_{N}$ is the location of the scatterer; $c_{o}$ the speed of sound. 


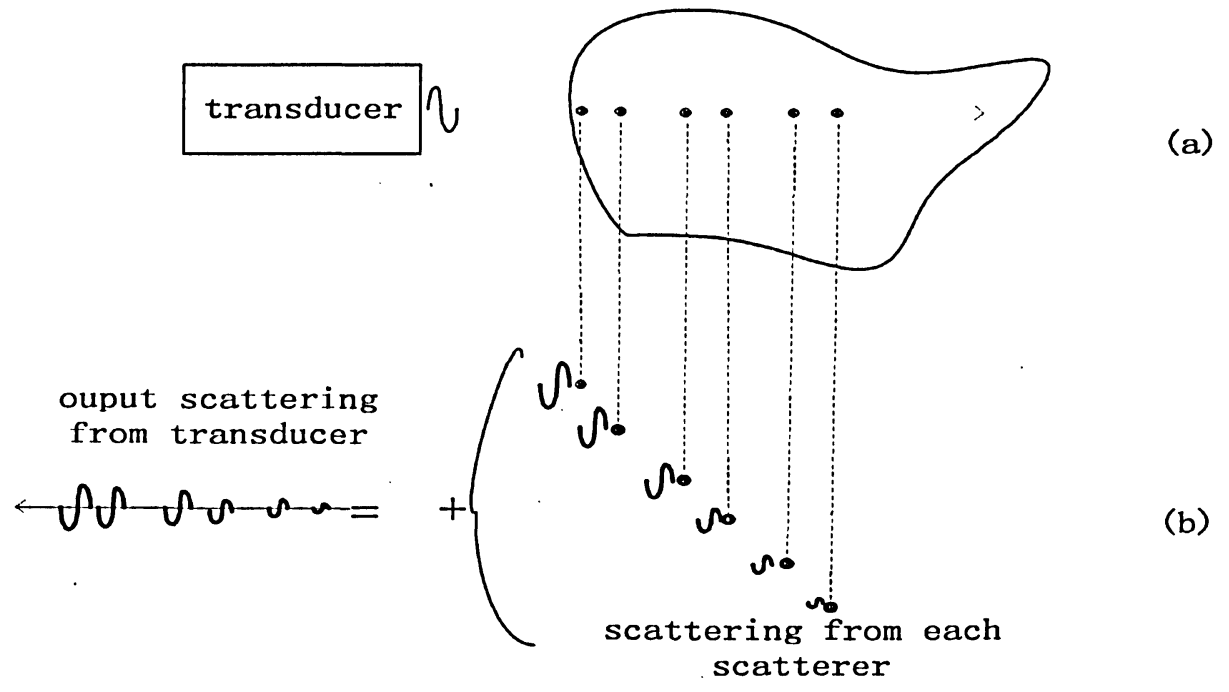

Figure 1.8 One-dimensional Scattering Model

\subsubsection{Our Research Interest ${ }^{[22]}$}
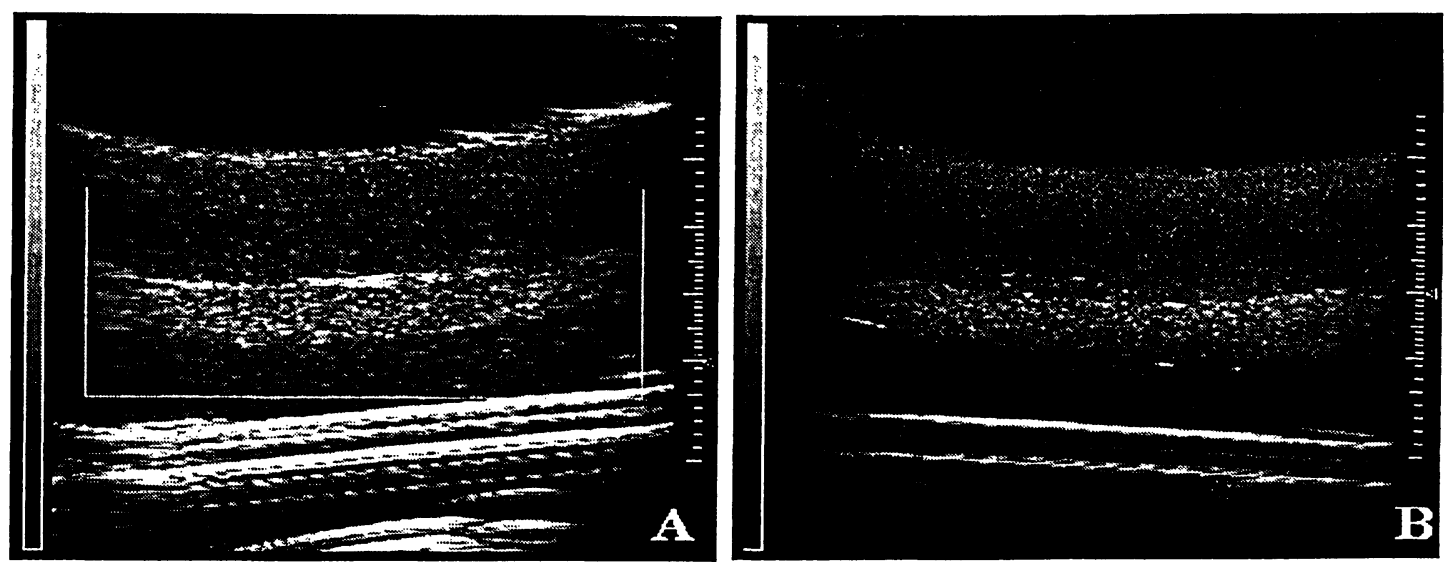

Figure 1.9 Normal-Treated-Normal Pellet of Cells Using the 20MHz (A) and 40MHz (B) Transducers. The Treated Layer can Clearly be

Differentiated due to the Backscatter Increase ( 11dB). H\&E Stains of the

Pellet from the Cisplatin Unexposed Top Layer ${ }^{122]}$

In some cases, the speckle of the ultrasound system is not noise. For example, we are interested in the scatterer patterns of different tissues. In this case the speckle is the signal we are working on. The cellular structure changes in tissues and cell 
ensembles can be detected by high frequency ultrasound system. The following are some of the results.

A three-layer pellet (untreated tissues, treated tissues and untreated tissues) is created to better visualize the contrast anticipated. The first (bottom) layer of the pellet contains unexposed cells, the second (middle) layer 24h cisplatin exposed cells and the third (top) layer unexposed cells. Figure 1.9A and 1.9B show the images collected using the $20 \mathrm{MHz}$ and $40 \mathrm{MHz}$ transducers, respectively. It is easy to identify the treated layers from the untreated layers. At $20 \mathrm{MHz}$ (Figure 1.9A), all of the cell layers are visible, but at $40 \mathrm{MHz}$ (Figure 1.9 B), the bottom layer is not visible because the ultrasound has been attenuated at the higher level. The histology of the pellet corresponds well with the ultrasound images.

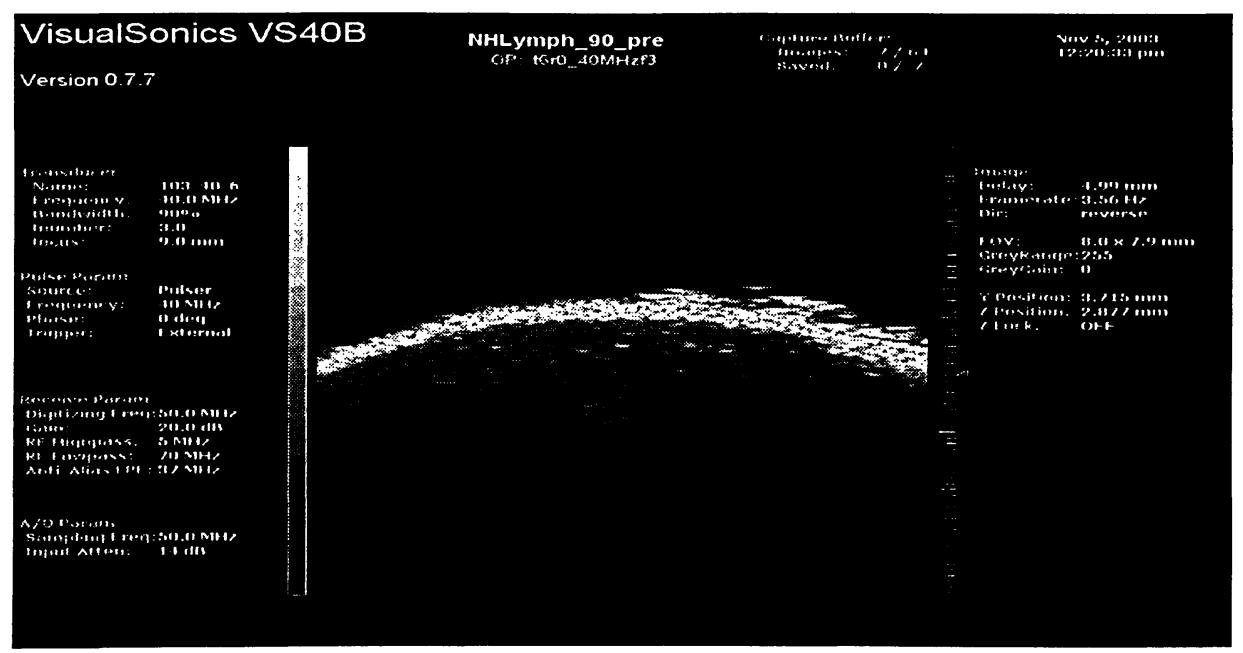

Figure 1.10 A Lymphoma Tumor in Mouse Leg 40MHz Transducers. ${ }^{[22]}$

In Figure 1.10, a lymphoma tumor is injected in the leg of a mouse, but the limit penetration depth prevents us from seeing the whole tumor. We hope to find a solution to increase signal-to-noise ratio to extend the penetration depth. The bright layer on the top is the skin. 


\subsection{Ultrasound System}

Basic concepts of an ultrasound system will be presented here. It is our goal to devise methods to increase the signal-to-noise ratio in high frequency ultrasound applications. The coded excitation method is a possible solution to increase the penetration depth of high frequency ultrasound system and is helpful for us extend our study on the high frequency backscatter properties of tissues.

\subsubsection{The Structure of an Ultrasound System}

In order to determine the feasibility of using the binary coded excitation method, we constructed a new system based on what was used in our experiment.

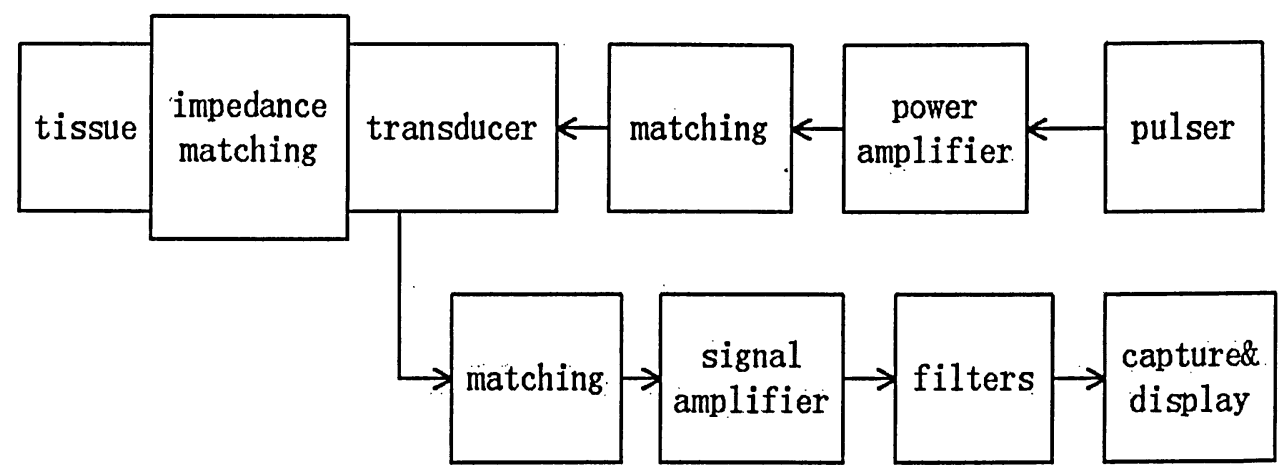

Figure 1.11 The Structure of Ultrasound System

In Figure 1.11, the core of ultrasound system is the transducer. It can be used to generate ultrasound signal and receive the backscatter signal. There are two parts in the figure: the top part of the figure is used to generate the ultrasound signals; the lower part is used to receive the backscatter signal. First a pulser generates a small electrical signal and the signal is sent to a power amplifier to amplify the 
signal to be suitable to drive the transducer. A matching circuit between the amplifier and the transducer is used to match the input impedance of transducer with the output impedance of the power amplifier and to cut the noise of the power amplifier from the receiver circuit. Then the transducer sends out an ultrasound signal. The ultrasound signal needs some materials such as water to match the mechanical impedance between transducer and tissue. At the same time, the backscatter signal will trace back on the same path to the transducer. This time the transducer converts the mechanical energy to an electrical signal; the signal passes a matching circuit that matches the output impedance of transducer with the input impedance of the signal amplifier. After the signal amplifier amplifies the signal, the signal passes several filters to remove noise. In the end, the signal is captured and displayed after proper processing.

The most important thing of setting up an ultrasound system is selecting the proper transducer. The frequency, the material and the focus are considered carefully. After the transducer is decided, the proper power amplifier can be decided on considering the gain, the frequency, input and output characteristic. Then the matching circuit can be designed. The proper pulser is chosen. The design of signal generator of system is finished. For the receiver, the signal amplifier should be decided on considering the gain, the frequency, and input and output characteristic. After knowing the input impedance of signal amplifier and the output impedance of the transducer, we can complete the matching circuit design. The proper filters are used to remove the noise from the signal. Last thing to decide are the proper capture \&display instruments, the sampling rate, the accuracy of the $\mathrm{A} / \mathrm{D}$, the store capability and sampling control. 


\subsection{Coded Excitation}

\subsubsection{Introduction}

Coded excitation methods have been developed since the 1950's, initially for use in radar to obtain improved range/resolution, and subsequently in communications systems. Later the application of coded excitation methods for improving the performance of ultrasound systems was initially explored for NDE (non-destructive testing) applications and has recently been developed for medical diagnostic systems.

In pulse echo systems the signal-noise ratio can be improved by increasing the transmitter peak power and increasing the bandwidth can enhance the axial resolution. The limitations imposed by the transmitted peak power were first addressed in radar and, stimulated in 1953 by Woodward, methods were developed for overcoming it. It was shown that by transmitting a suitably encoded signal of over longer time duration and using a receiver that incorporated a matched filter (one whose response was the time reversed form of the transmitted signal), a time-compressed form of the transmitted signal could be produced. By spreading the transmitted signal over a longer duration a higher total energy can be transmitted without exceeding the peak power limitation and as a result, the depth of penetration and the signal-to-noise ratio can be increased. For ultrasound systems issues such as the permitted diagnostic levels and nonlinear effects limit the peak power that can be transmitted. Major signal-to-noise ratio improvements can be achieved by using coded excitation techniques and, some of which have been developed for low frequency medical ultrasound imaging. 


\subsubsection{The theory of coded excitation}

Figure 1.12 compares a coded excitation system and a conventional pulse system. In a coded system, the excitation pulse extends over a period longer than the impulse response of the transducer. The excitation sequence includes all frequency components passed by the transducer. Because of this, the received signal can be correlated with a reference waveform to yield a compressed signal with impulse response closely approximating that of the normal pulsed system. The major advantage is that the SNR of the correlated pulse is improved over that of the normal pulse by a factor close to the time bandwidth product of the coded excitation sequence, while the instantaneous peak power keeps the same value.

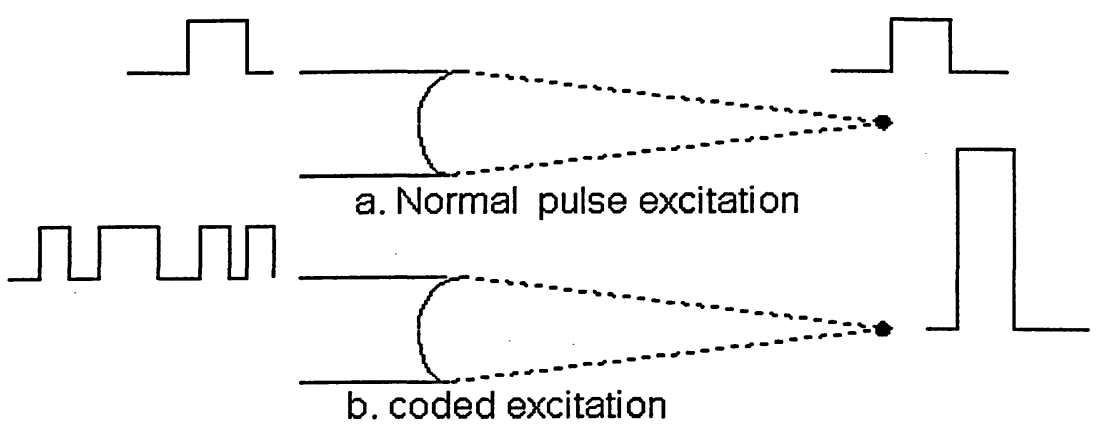

Figure 1.12 Conventional Pulse and Coded Excitation ${ }^{[34]}$

Coded excitation is similar to sensitization in photography. The total energy of light exposure needed by the photographic film is in a certain dosage range. When the intensity of the light is high, exposure time is short. If the intensity of light is low, a longer time is required for the exposure. If the intensity of light is constant, the total energy of light on the photographic film is decided by the exposure time. The longer exposure time, more energy received; the shorter time, less energy collects on a film. The only different between coded excitation method and sensitization is how to "accumulate" the energy. The photons of light are accumulated and collected by the photographic film directly; where coded 
excitation completes this process by encoding and decoding the signal, the result of encoding and decoding the signal will compress the signal.

Now let us see how coded excitation methods are implemented. Generally cross-correlation methods are used in a coded excitation system. If two input signals $s_{i}(t)$ and $t_{\tau}(t)$ are cross correlated, the output of the cross-correlation $s_{o}(t)$ is given: $s_{o}(t)=\int_{-\infty}^{\infty} s_{i}(t+\tau) t_{\tau}(\tau) d \tau$. Assume that the pulse echo impulse response of a given transducer denoted by $h(t)$. If the transducer is excited by a waveform $e_{t}(t)$, then the received waveform $e_{r}(t)$ is given by:

$$
e_{r}(t)=e_{t}(t) * h(t)=\int_{-\infty}^{\infty} e_{t}(t-\tau) h(\tau) d \tau
$$

If this is sent to a receiver system whose impulse response is $h_{r}(t)=e_{r}(t)=k e_{t}(-t)$, the output of such system is the autocorrelation of the transmitted signal. Where $k_{m}$ is a matched filter constant, then, the output from the receiver will be given by

$$
\begin{aligned}
e_{o}(t) & =\int_{-\infty}^{\infty} e_{t}(t+\xi) e_{r}(\xi) d \xi \\
& =\int_{-\infty}^{\infty} e_{t}(t+\xi) * k_{m} e_{t}(\xi) d \xi \\
& =R_{e e}(t) k_{m}
\end{aligned}
$$

$e_{o}(t)$ the output from encoded system; $e_{t}(t)$ waveform excitation; $e_{r}(t)$ received waveform; $R_{e e}(t)$ is the autocorrelation of the transmitted signal. In the frequency domain, the relation between the receiver matched filter transfer function $H(\omega)$ and the input signal $E(\omega)$ is: $H(\omega)=k E^{*}(\omega), E^{*}(\omega)$ is the complex conjugate of the input signal.

A conventional ultrasound system and a coded excitation ultrasound system can be expressed as Figure 1.13(a) and 1.13(b). The difference between these two systems is that in coded excitation system a correlation operation of the received signal is required to compress the encoded signal. It is equivalent to a system 
whose impulse response is received signal $e_{r}(t) .\left(h_{r}(t)=e_{r}(t)\right)$

(a)
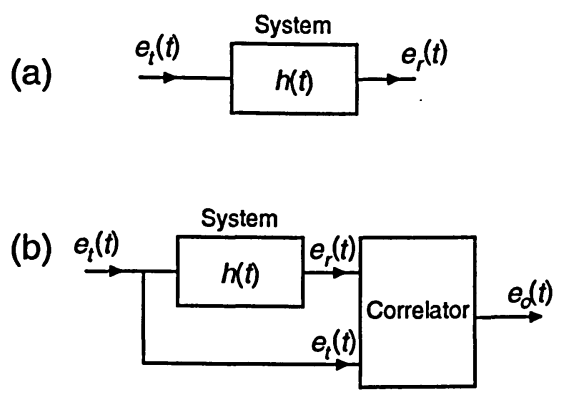

Figure 1.13 Conventional Systems and Coded Excitation Systems ${ }^{[6]}$

How does the autocorrelation operation work? For example, a code sequence 11001110 is chosen to shown the output of autocorrelation of the sequence. As Figure 1.14 (a) and (a1) shows, before the autocorrelation the amplitude of the signal is one (Figure 1.14 (a)), but after correlation the peak amplitude is 5 (Figure 1.14 (a1)). In Figure 1.14 (a) we used values of 0,1 to express the code (code: 11001110) and in Figure 1.14 (b) the values of 1 and -1 are used to express the code (code: 11-1-1111-1), so its correlation peak amplitude is 9 (Figure 1.14 (b1)). The former is called uni-polar modulated method; the latter, called bipolar modulated method, has greater output than the first one. In communication theory, the bipolar method is more efficient encoded method than uni-polar or multi-phase methods. From the autocorrelation output, a series of sidelobes exist on the both side of the peak value. The values of sidelobes are different for different code sequences. If we choose a proper code, the sidelobes can be controlled. 


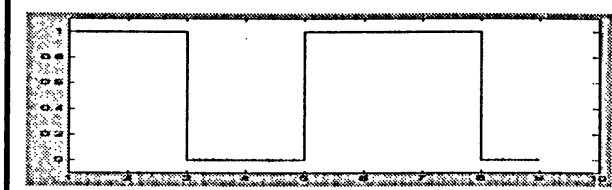

(a) uni-polar code

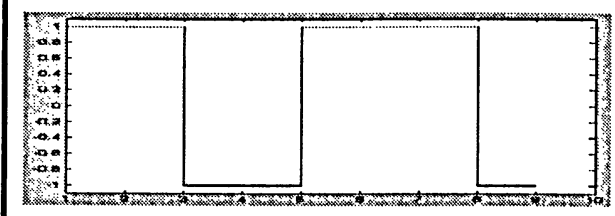

(b) Bipolar code

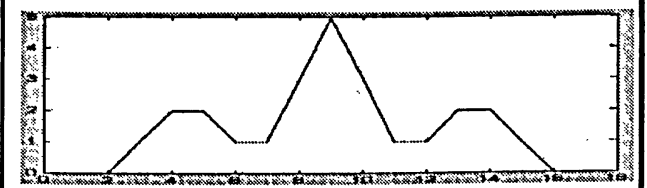

(a1) correlation of uni-polar code

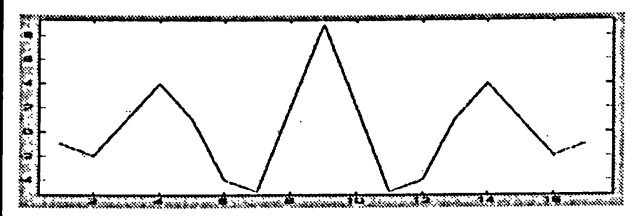

(b1) correlation of bipolar code

Figure 1.14 Encoded Method and Autocorrelation

\subsubsection{Two Coded Excitation Methods}

In high frequency ultrasound imaging tissue imaging, the presence of high attenuation and non-stationary targets reduces the possible types of encoding schemes that can be employed but some still retain the potential for achieving significant SNR improvements. Two different schemes that have been explored for use in low frequency diagnostic ultrasound systems will be examined in details: the FM chirp and Golay binary codes. These two methods are classified by type of modulated signal. The modulated signals can be a pulse, a piece of continuous signal and a piece of frequency-modulated sweep. When the modulated signals are a piece of frequency-modulated sweeps, this method is called FM chirp. When the modulated signal is pulse or a piece of continuous signal, this method is generally Binary coded excitation method.

\subsubsection{FM Chirp Coded Excitation}

FM chirp coded excitation applications were first used in radar. This is a more popular coded excitation method than binary coded excitation. Articles have been

published by Matthew O'Donnell's ${ }^{[34]}$, N. Rao's ${ }^{\text {[39][40|[411][42]|43], Thanassis X }}{ }^{[30 \mid[31]}$ on this method. The main advantage of this method is that it can improve the 
resolution and sensitivity. It is one reason why it is popular, another one is that it is used in radar systems and is easily implemented in ultrasound imaging system.

The product of time bandwidth is the theoretically attainable signal-to-noise (SNR) enhancement. For example $10 \mu \mathrm{s}$ FM chirp with a center frequency $10 \mathrm{MHz}$ and a bandwidth of $5 \mathrm{MHz}$. The time-bandwith is 50 times greater than a simple pulse echo system.

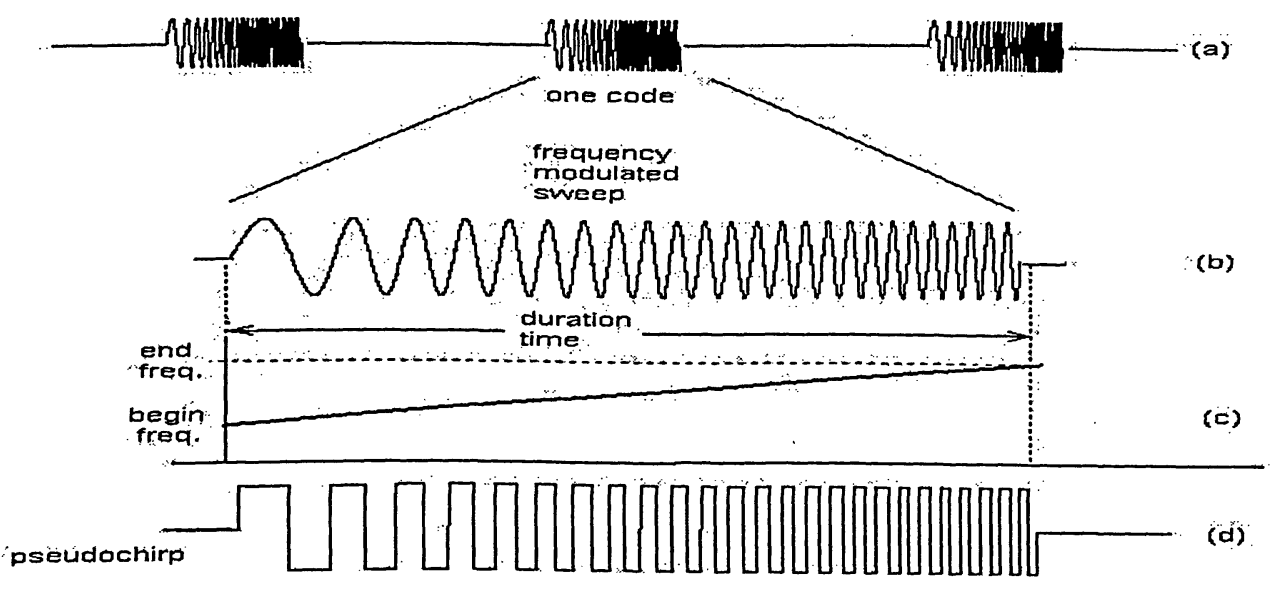

Figure 1.15 FM (Frequency Modulated) Chirp ${ }^{[6]}$

Figure 1.15 (a) represents a series of frequency modulated sweep signals that are encoded by a special code. Figure 1.15 (b) denotes one region of the frequency modulated sweep signal. The frequency of signal changes with time; the range of change is shown in the third line. As Figure 1.15 (c) the frequency rises from the begin frequency to the end frequency during the duration time of signal. Generally a piece of frequency modulated signal is expressed as below:

$$
s(t)=\operatorname{rect}\left(\frac{t}{T}\right) s_{0} \cos \left(\omega_{0} t+\frac{\alpha}{2} t^{2}\right)
$$

where $T$ is the duration time of FM chirp, $s_{0}$ is the attenuation factor, $\omega_{0}$ is the angular frequency of the carrier, and $\alpha$ sweep rate is the slope of the linear chirp (Figure1.15(c)) $\alpha=\frac{f_{\text {end }}-f_{\text {begin }}}{T}$. $f_{\text {end }}$ is signal frequency at the end time of 
signal duration $T$, and $f_{\text {begin }}$ is the signal frequency at the beginning of the modulated signal.

A coded excitation sometimes called pseudochirp is a close approximation to the ideal frequency modulated sweep signal as Figure 1.15 (d). This signal is easy to generate in a digital system.

Newjouse and Pedersen first suggested this implementing method of FM chirp used for medical purpose in 1973 (Figure1.16) and Wilhjelm and Pedersen implemented such a scheme in 1993. First a code generator generates the code and the sweep generator generates the frequency-modulated chirp. The modulator modulates both of signals; the FM chirp signal looks like Figure 1.15 (a). The FM chirp is sent to transducer through a power amplifier. At the same time the signal is sent to a time delay device. The backscatter signals of FM chirp signal are returned from tissue and received by the receiver. An amplifier amplifies the signal. In the end, the delayed FM chirp signal subsequently cross-correlates with the received backscatter signal of FM chirp.

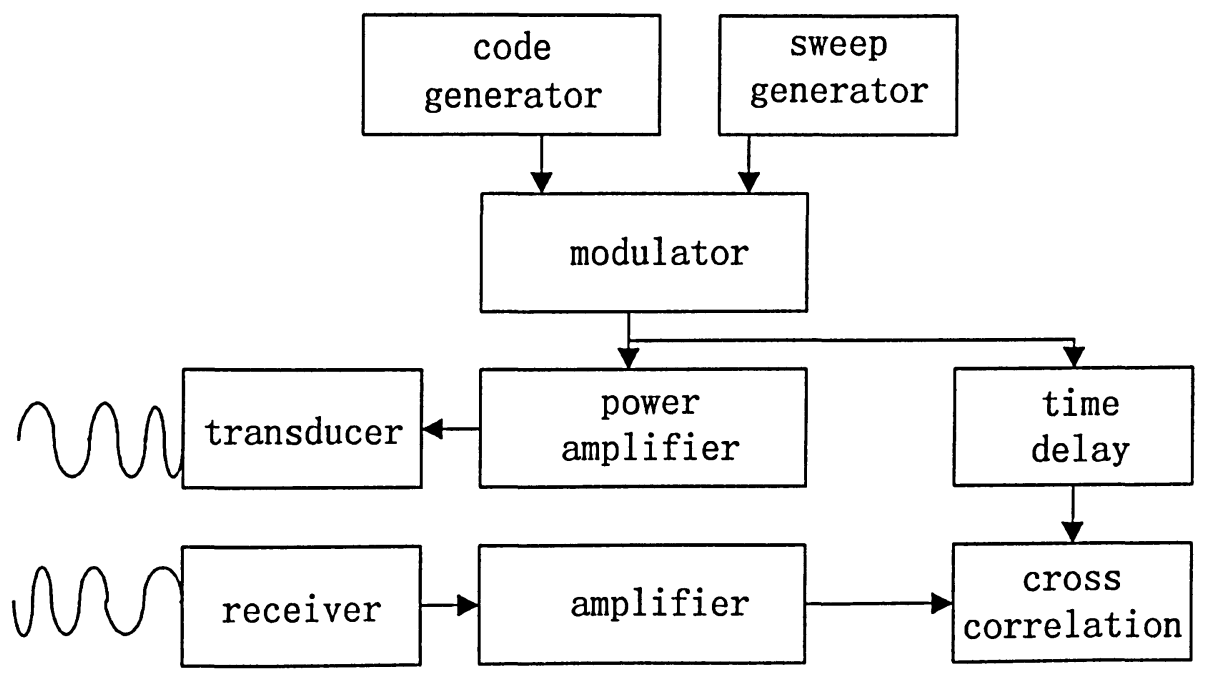

Figure 1.16 Implementation of FM Chirp

From equation (1.27), the FM chirp signal can be expressed as

$$
s(t)=\operatorname{rect}\left(\frac{t}{T}\right) s_{0} \cos \left(\omega_{0} t+\frac{\alpha}{2} t^{2}\right)
$$

To simplify the problem, assume attenuation factor $s_{o}$ is 1 , and that the matched 
filter is

$$
h(t)=\operatorname{rect}\left(\frac{t}{T}\right) s_{0} \cos \left(\omega_{0} t-\frac{\alpha}{2} t^{2}\right)
$$

The output of the filter is

$$
e_{o}(t)=\int_{-T / 2}^{T / 2} \operatorname{rect}\left(\frac{t}{T}\right) \cos \left(\omega_{0} \tau+\alpha \tau^{2} / 2\right) \cos \left[\omega_{0}(t-\tau)-\alpha(t-\tau)^{2} / 2\right] d \tau
$$

By using the trigonometric expression for the products of cosine terms, and ignore the frequency is $2 \omega_{0}$ the result is:

$$
e_{o}(t)=\operatorname{rect}[t /(2 T)] \cos \left(\omega_{0} t\right) \frac{\sin [\alpha t(T-|t|) / 2]}{\alpha t}
$$

The duration time of output extends over twice the initial chirp duration.

A FM chirp, as shown Figure 1.17, is consisting of a 10 $\mu$ s FM chirp with a center frequency of $5 \mathrm{MHz}$ and a bandwidth of $2 \mathrm{MHz}$. Such a waveform has time-bandwidth product that is 20 times larger than a simple pulse echo system.

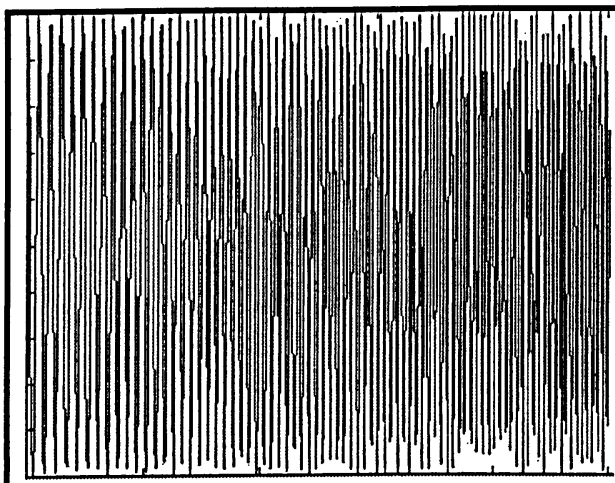

(a) FM Chirp

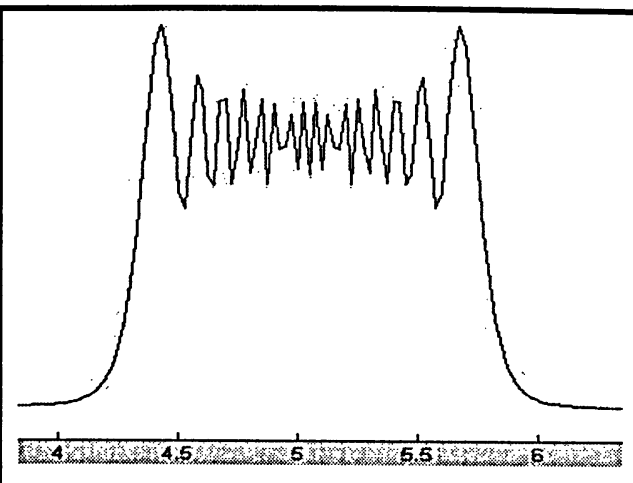

(b) FM Chirp in Frequency Domain

Figure 1.17 FM Chirp ${ }^{[35][6]}$

The compressed signal is shown in Figure 1.18. It can be seen that the compressed form contains range sidelobes. Compare the amplitudes of sidelobe to that of the main lobe and the difference between them will decide how difficult it is to identify the presence of multiple scatterers. When a weakly scatterer is near a 
strongly one, the main lobe of the weakly one is less than the sidelobe of the strong one. So that it may be difficult to distinguish from the range sidelobes of the strongly scattering target. Much effort has been devoted to reduce sidelobes of compressed signal. A method that reduces the sidelobes is binary coded excitation.

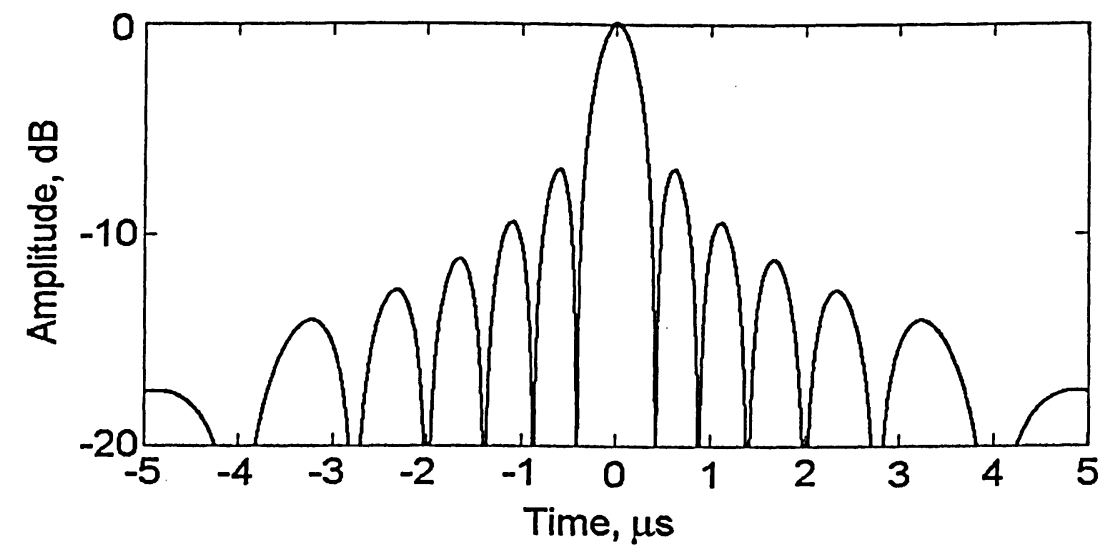

Figure 1.18 The Compressed Signal ${ }^{[34 \mid[6]}$

\subsubsection{Binary Coded Excitation}

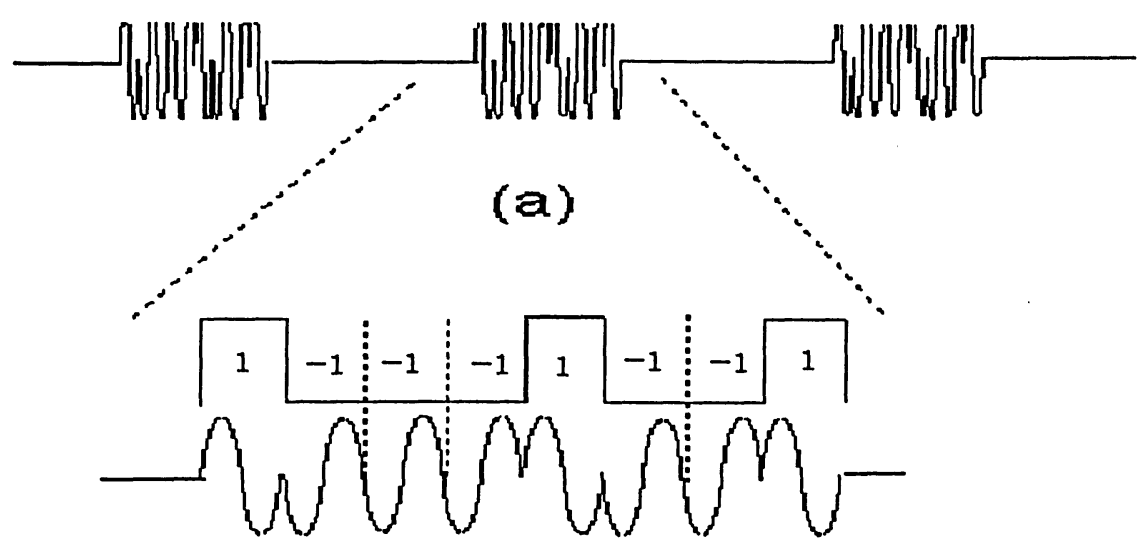

Figure 1.19 Binary Coded Excitation ${ }^{[6]}$

As in Figure 1.19, a sequence of one cycle sine signal is coded by a binary code.

When code is 1 , one cycle of sine is used; when the code is -1 , one cycle of 
inverse sine is chosen. This bipolar method can maximize the energy of code. This method is chosen in my thesis, as few people have tested this method before. This method cannot improve the resolution, but it may be a solution to gain higher SNR than FM chirp methods.

In the FM chirp system, when we want to maximize the peak signal-to-noise ratio, the method called the matched filter is used. The transfer function of the matched filter is the complex conjugate of the signal spectrum. In a real system, especially in medical systems, the effect of frequency dependent attenuation cannot be ignored; the attenuation will increase greatly with higher frequency. Many experts believe that frequency dependent attenuation is the major reason why coded excitation application in the ultrasound field is fewer compared with that in radar ${ }^{[34][6]}$. In this case, some mismatched filters will be introduced in signal processing, for example Dolph Chebyshev window and Blackman Harris window ${ }^{[27][30]}$. Both of the filters can lower sidelobe levels.

\subsubsection{Properties of Golay Code and Golay Code Pairs}

Golay codes can be written as finite sequences of 1's and 0's and they have the special property that their autocorrelation function can contain only three possible values: 0,1 and $N$, where $N$ is number of bits in the code. Figure 1.20 shows the 8-bit Golay code 1110010. If we assume that the transmitted signal is such that each bit has a duration of 1 second, it can be readily shown that the autocorrelation function consists of a central lobe of 2 seconds duration and a peak amplitude that is seven times larger than the side lobes. Compared to the other types of codes, the Golay code sidelobe energy is small. 

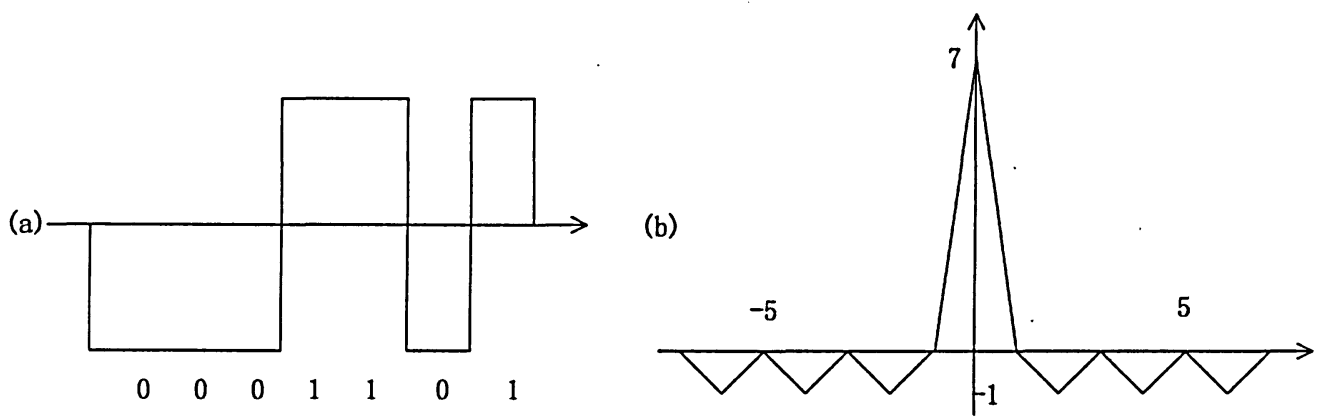

Figure 1.20 Golay Code and Its Autocorrelation

A method can be used to eliminate the sidelobes using Golay code pairs. Figure 1.21 shows Golay code pairs of code length 8 . The sidelobes of autocorrelation Golay code and its complementary Golay code have the same amplitude and inverse phase, so the sum of these two autocorrelations is delta function. This property is helpful in reducing the sidelobe of the system, so Golay codes are chosen for my thesis.
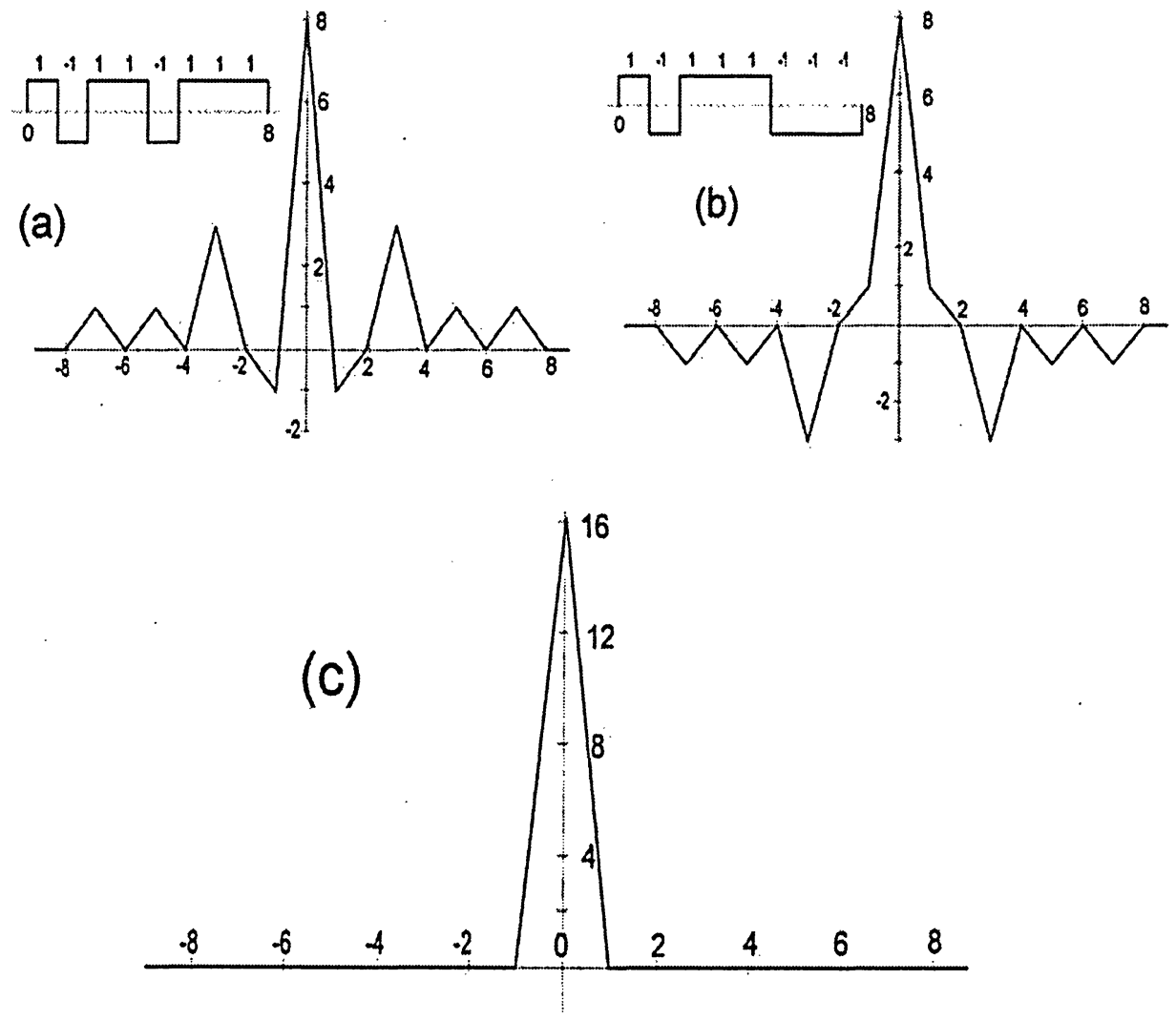

Figure 1.21 Golay Code Pairs ${ }^{[6]}$ 
All the signals are processed following the same procedures as described in Figure 1.21. The backscatter signals from the encoded Golay signal and its reference cross correlate, so as the backscatter signals from the encoded complimentary Golay signal and its reference. We add these two middle results together to obtain the final decoded signals. These results are called encoding results.

As in Figure 1.22, GE company manufactures a kind of digital coded excitation and compression ultrasound system (proprietary). This system has shown that significant improvements in penetration depth and SNR can be achieved clinically.

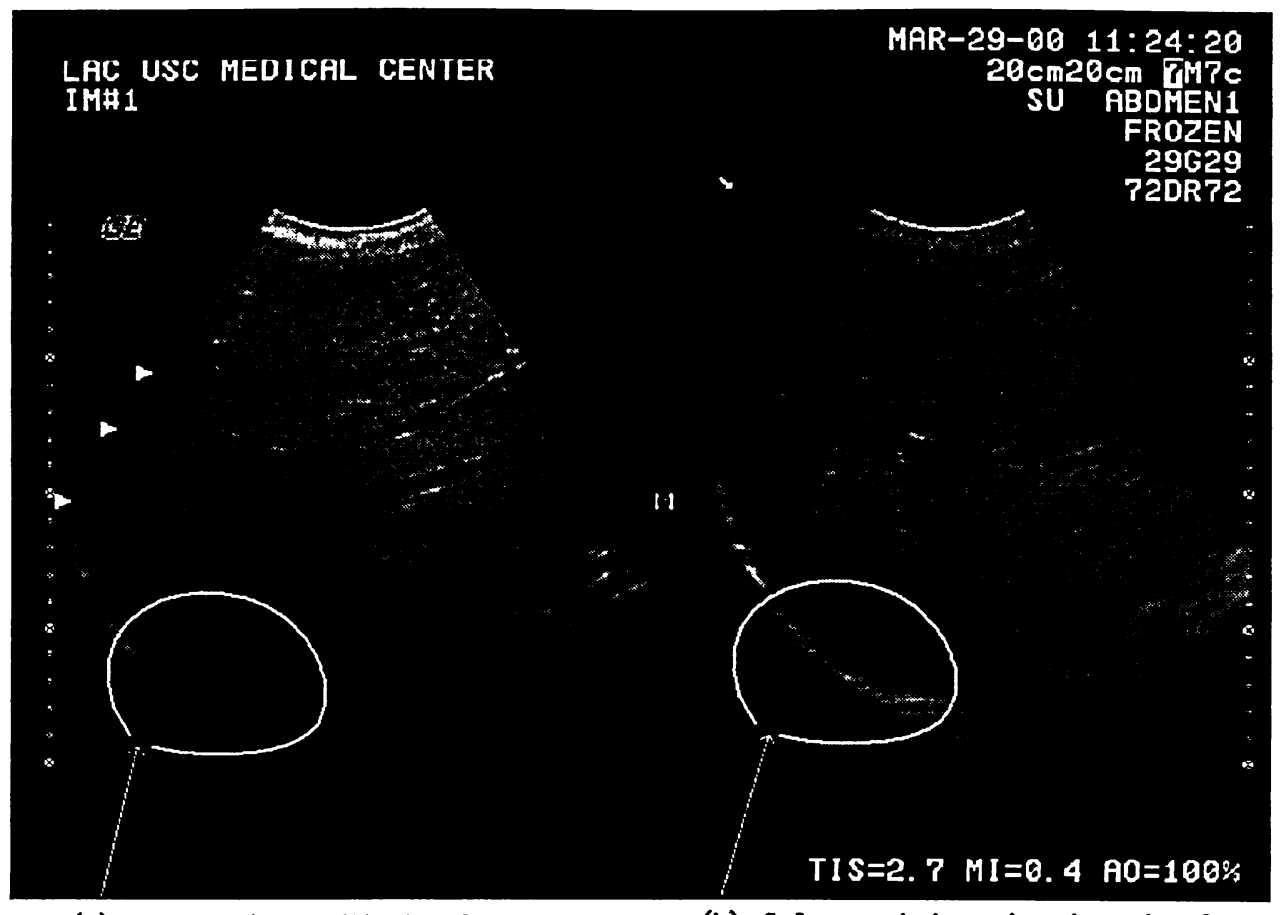

(a) conventional US signal.

(b) Golay coded excitation signal

Figure 1.22 Golay Coded Excitation Signal and Conventional Signal The Benefits of Coded Excitation are Clearly Demonstrated in this Side-by-side Liver Comparison. Note the 20-cm Penetration while Maintaining 7-MHz Resolution

Sources** http://www.gemedicalsystems.com/rad/us/products/log 700pro/msu585.html As marked in Figure 1.22, the high signal-to-noise ratio using the coded excitation method in Figure 1.22 (b) allows us to identify the rim of the liver tissue easily, 
while the rim of the liver signal in conventional ultrasound system at same depth is not easy to distinguish in Figure 1.22 (a).

\subsubsection{The Components of a Binary Coded Excitation System}

The coded excitation ultrasound imaging system has several parts: signal generator, code generator, modulator, transducer, receiver and decoder (Figure 1.23). First the ultrasound signal and codes signal are generated, then a modulator modulates them, then coded signals are sent out by transducer into the tissue, and the backscatter signals are received by the receiver, in the end these received signals are decoded by the decoder. In our system, we use golay code and complimentary golay code to modulate the original ultrasound signals, because golay code is the most useful code for coded excitation by suppressing the sidelobes. We know that a code with a $\delta$-function autocorrelation is an ideal one for imaging. For any practical code, the duration is restricted so that a $\delta$-function autocorrelation function can never be obtained, but if we use golay code and complement golay code together, then the added autocorrelations of these two codes is very similar to a $\delta$-function (Figure 1.21). This will be useful to reduce the range side-lobes. 


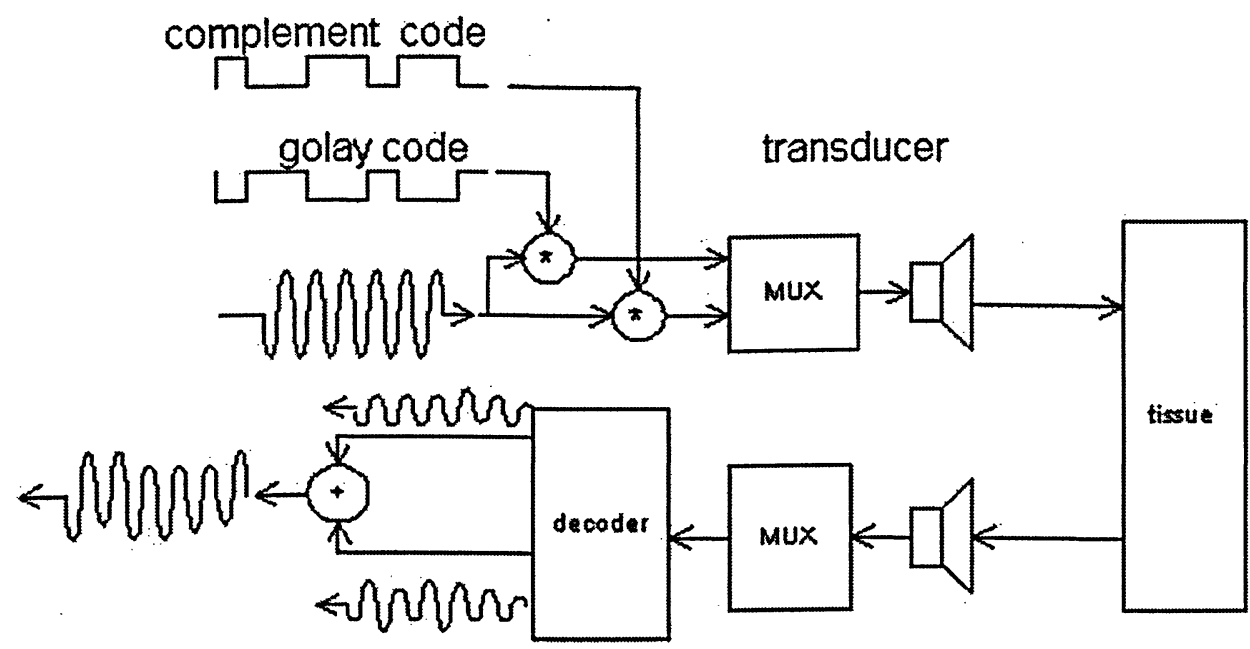

Figure 1.23 Coded Excitation System

In this chapter we introduce almost all the basic concepts in ultrasound system and coded excitation method. From now on we will discuss simulation and implementation of binary coded excitation method in high frequency ultrasound system. Some conclusions are made based on the results from our simulations and experiments. 


\section{Chapter 2 Methodology}

\section{$2.1 \quad$ Introduction}

There are two parts in this chapter. In the first part backscatter simulations for high frequency ultrasound using a coded excitation system are presented. In second part we describe the experimental system built to implement the binary coded excitation method.

\subsection{Simulation of Coded Excitation}

The structure of a binary coded excitation system is introduced in Section 1.10.5. A binary coded excitation system includes: a code generator, a signal generator, a modulator, a transducer, a receiver and a decoder. The function of the code generator, signal generator and modulator is to generate binary coded excitation signals. The function of transducer and receiver is to generate a signal and receive the backscatter signal from the object we are interested in. The function of last part, decoder, is to process the received signal.

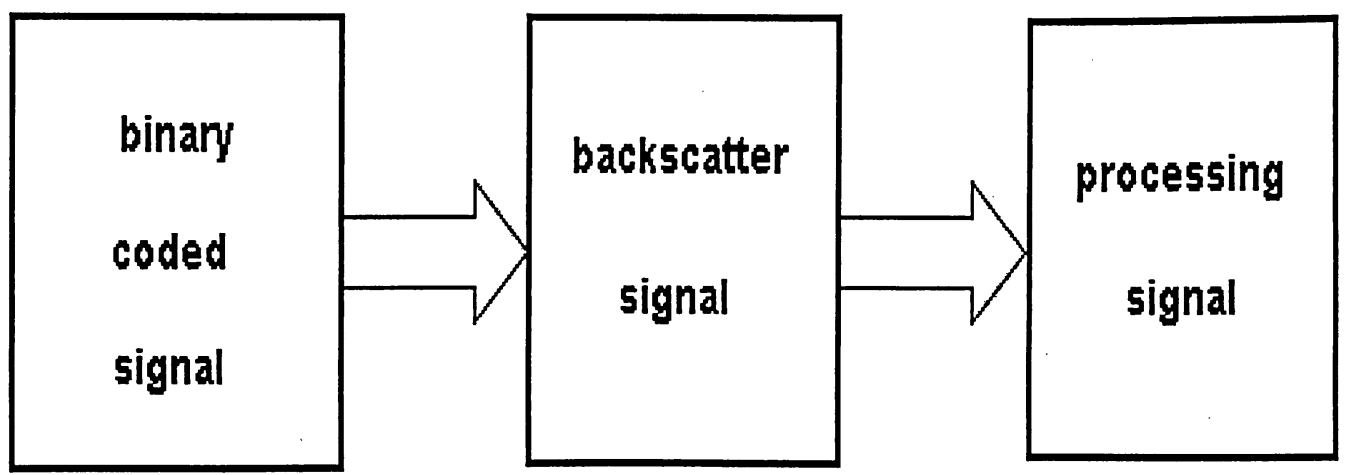

Figure 2.1 The Structure of Simulation

Figure 2.1 demonstrates the three parts needed to simulate the binary coded 
excitation method; these three parts correspond to the three different functions of the coded excitation system.

In the binary coded signal section, we will discuss how to generate a binary coded signal in detail. The questions such as the type of codes, how to get the codes, the signals we use to simulate and how to modulate them will be answered in this section.

In the backscatter signal section, we will discuss how to model the backscatter signal. The backscatter signals have direct relation to transducer, tissue and the medium that ultrasound signals travel into. The questions such as how to implement one-dimensional backscatter model, how to take into account tissue attenuation, how to simulate spacing of scatterers, will be addressed. In the signal processing section, we will discuss the different methods to process the received coded signals. In the last section we will present some basic concepts that will be used to evaluate our results and draw conclusions.

\subsubsection{Encoded Signal}

In order to generate binary coded excitation signal, four questions are required to be answered clearly: What kind of codes are selected? How is the code generated? What kinds of codes are used? What type of modulator is chosen? The modulator is discussed in Section 1.10.2.

\subsubsection{Golay Codes}

The Golay code pairs are discussed in Section 1.10.4. The autocorrelations of Golay pairs have sidelobes with the same amplitude and inverse phase, so that their sum has no sidelobes. The smaller the sidelobes, the better the system resolution is. In our system the Golay code pairs are chosen. 
Marcel J. E. Golay first published the Golay code pairs in 1951. We will investigate the effects of different code length. How can we get the Golay code pairs? Let us see the definition and properties of Golay code pairs.

\subsubsection{Code Generation}

The definition of Golay code pairs: a set of complementary series is defined as a pair of equally long, finite sequence of two kinds of elements which have the property that the number of pairs of like elements with any given separation in one series is equal to the number of pairs of unlike elements with the same separation in the other series ${ }^{[13]}$.

Figure 2.2 illustrates a pair of complementary Golay sequences. We can demonstrate the symmetry of Golay code pairs' definition from this example. Sequence 1 has four pairs of like elements with separation of one; three pairs of unlike elements with separation one. Whereas Sequence 2 has 4 pairs of unlike elements with separation of one; and three pairs of like elements. Table 2.1 summarizes the results of the element pairing for separation 1,2 , and $3^{* *}$.

From the analysis, we can see complementary codes possess symmetry. We can use this property to generate different Golay code pairs. Assume $a$ and $b$ are Golay code pairs. If we concatenate these two pairs as $c=[a, b], d=[a,-b]$, the results of cascade operation $\mathrm{c}$ and $\mathrm{d}$ are still Golay complementary code pairs. In this case we can get different code length very easy. This symmetry makes code generation straightforward.

\footnotetext{
"* Source: Complementary Code Keying Made Simple (Bob Pearson)
} 


\section{SEQUENCE 1}

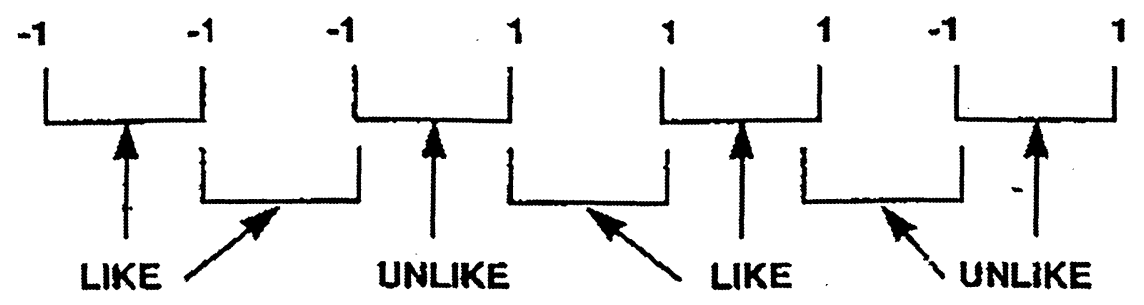

SEQUENCE 2

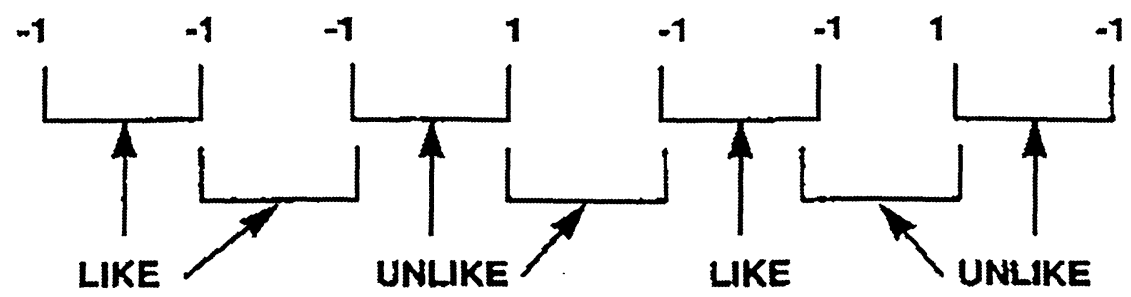

Figure 2.2 Golay Code Pairs

Source: Complementary Code Keying Made Simple (Bob Pearson)

Table 2.1 Results of Element Pairing for Sequence 1 and Sequence 2

\begin{tabular}{|c|c|c|c|c|}
\hline \multirow{2}{*}{$\begin{array}{c}\text { PAIR } \\
\text { SEPARATION }\end{array}$} & \multicolumn{2}{|c|}{ SEQUENCE 1 } & \multicolumn{2}{c|}{ SEQUENCE 2 } \\
\cline { 2 - 5 } & LIKE & UNLIKE & LIKE & UNLIKE \\
\hline 1 & 4 & 3 & 3 & 4 \\
\hline 2 & 4 & 3 & 3 & 4 \\
\hline 3 & 1 & 5 & 5 & 1 \\
\hline
\end{tabular}

Source: Complementary Code Keying Made Simple (Bob Pearson)

The code lengths are two, four, eight and sixteen respectively. The Golay complementary pairs for code length two are: $\{1,1\}$ and $\{1,-1\}$ or $\{-1,-1\}$ and $\{-1,1\}$. Beginning with these two pairs we can construct two groups, the two 
groups of Golay complementary pairs for code lengths two four, eight and sixteen are:

First group of Golay code pairs

Code length two $\quad\{1,1\}$ and $\{1,-1\}$

Code length four: $\quad\{1,1,1,-1\}$ and $\{1,1,-1,1\}$;

Code length eight: $\quad\{1,1,1,-1,1,1,-1,1\}$ and $\{1,1,1,-1,-1,-1,1,-1\}$;

Code length sixteen: $\{1,1,1,-1,1,1,-1,1,1,1,1,-1,-1,-1,1,-1\}$ and

$$
\{1,1,1,-1,1,1,-1,1,-1,-1,-1,1,1,1,-1,1\}
$$

Second group of Golay code pairs

Code length two: $\quad\{-1,-1\}$ and $\{-1,1\}$

Code length four: $\quad\{-1,-1,-1,1\}$ and $\{-1,-1,1,-1\}$

Code length eight: $\quad\{-1,-1,-1,1,-1,-1,1,-1\}$ and $\quad\{-1,-1,-1,1,1,1,-1,1\}$

Code length sixteen: $\quad\{-1,-1,-1,1,-1,-1,1,-1,-1,-1,-1,1,1,1,-1,1\}$ and

$$
\{-1,-1,-1,1,-1,-1,1,-1,1,1,1,-1,-1,-1,1,-1\}
$$

The length of the focal volume $\mathrm{Dz}$ of ultrasound field (Section 1.5.4) will limit the maximum code length. If the code length is longer than the time that signals pass focus depth, it means only part of code is in the focus region. The part out of focus region will be weak and have less contribution to the signal received (Figure 2.3). For the transducer used in the experiment, the diameter is $12 \mathrm{~mm}$; the center frequency is $40 \mathrm{MHz}$; the radius of the curvature is $10 \mathrm{~mm}$, so the $\mathrm{Dz}$ is about $2 \mathrm{~mm}$. Assuming a sound speed of $1.5 \mathrm{~mm} / \mu \mathrm{s}$, the backscatter signals pass such distance in $2 \times \mathrm{Dz} / 1.5=2.7 \mu \mathrm{s}$. The center frequency of our signal is $40 \mathrm{MHz}$, therefore the period of our signal is $1 / 40 \mathrm{M}=0.025 \mu \mathrm{s}$. Due to the transducer inertia, the signal cannot be sent out continuously. Some time is required to restore the transducer. The time for one pulse is 6 to 7 times of period. So the time for one pulse is $6 \times 0.025=0.15 \mu \mathrm{s}$. The maximal code length is the time passes the length of focal area over time needed for one pulse. This can be expressed as: 


$$
L_{\text {code }}=\frac{T_{\text {focuselength }}}{t_{\text {percode }}}
$$

For our transducer, the maximal code length is $2.7 / 0.15=18$. For a different transducer, the number is different. Only this limit needs to be considered in high frequency ultrasound system (30-50 MHz or more) in the choice of code length.

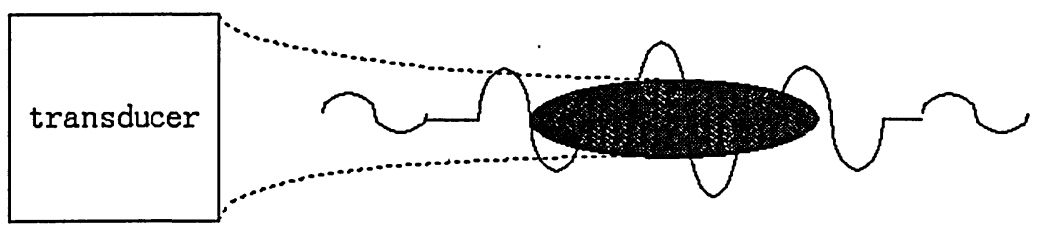

(a) focusing transducer

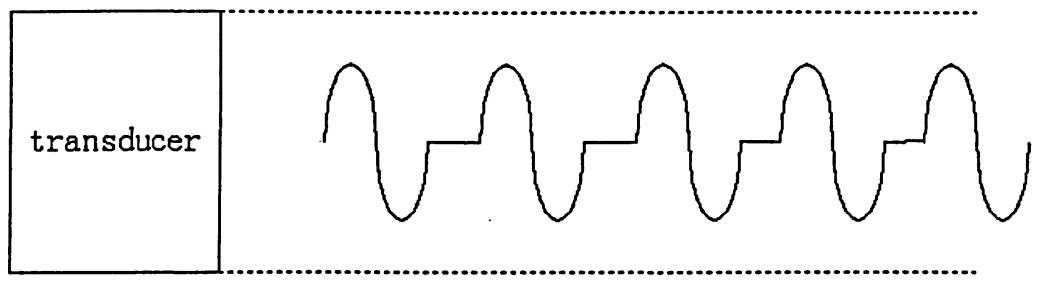

(b) unfocused transudcer

\section{Figure 2.3 Long Coded Signals in Focusing and Unfocused Transducer}

In Figure 2.3 (a), the black ellipse area represents the focus depth of a focused transducer. If the coded signal is longer than the depth of transducer, the pulses out of focus area are so weak that they have less contribution to the received energy by transducer. If the code is shorter than the focus area, it can be thought as an unfocused transducer. Therefore, all the coded pulses have the same contribution to the received energy by the transducer.

In our simulation we do not consider the focusing effect so that our simulation can be regarded as an unfocused system. We find the amplitude of backscatter signals increases with code length. In the experiments, code lengths of 2 and 4 show this trend. Code lengths of 8 and 16 show odd features as explained in Section 2.4.2. 


\subsubsection{Modulated Signal Type}

Three different types of modulated signal are used in our simulations. Initially we just used one cycle of sine signal (as in Figure1.21). In our system, the center frequency of transducer is $40 \mathrm{MHz}$, so the period of one cycle sine signal is $25 \mathrm{~ns}$.

Experimentally, we measure the emitted pulse using a reflection of a quartz plate as in Figure 2.4(a). In the second type of signal used, we emulate the pulse emitted by a real pizeoeletric transducer. The equation used to simulate the real system is

$$
p(t)=-t \exp \left(-4 \beta^{2} t^{2}\right) \sin \left(2 \pi f_{0} t\right) \quad t>0
$$

where $f_{0}$ is the center frequency of transducer $40 \mathrm{MHz}, \quad \beta$ is the $-3 \mathrm{~dB}$ bandwidth (different from attenuation). In the simulation, the signal lasts about 3 periods of center frequency ( Figure 2.4 (b) ).

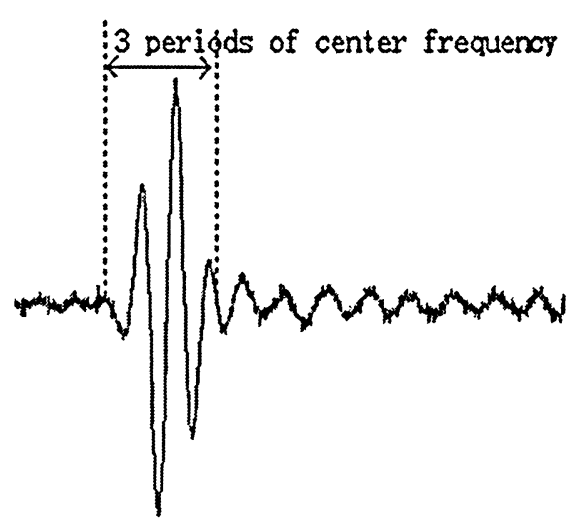

(a)

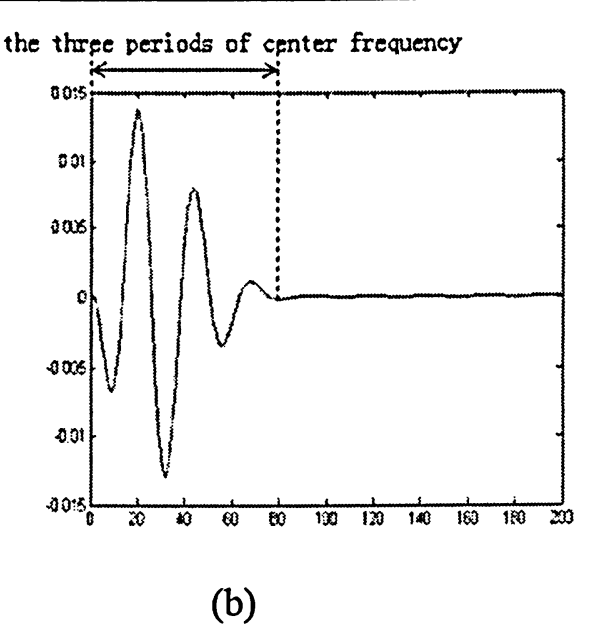

(b)

Figure 2.4 (a) Emitted Signal from Transducer (b) Simulation Signal

In both figures $\mathrm{y}$ axis is amplitude of signal and the $\mathrm{x}$ axis is the time in ns. Most of energy of emitted signal is in the three-cycle periods we annotate.

To better represent the experimental pulse, the second method to modulate the 
signal is a series of sine of signal modulated by $\exp \left(-4 \beta^{2} t^{2}\right)$ alone. If $\beta=4$, the signal lasts three periods. We use the first three period of the signal as the pulse response of system. One bit of Golay code corresponds to such pulse response. One modulated signal is shown as Figure 2.5 (a). In Figure 2.5 (a) the modulated signal for each bit of code comes from the three periods in Figure 2.4(b). The modulated signal in Figure 2.4(b) is sine signal modulated by the factor exp (-4 $\left.\beta^{2} t^{2}\right)$. In order to see the effect of $\exp \left(-4 \beta^{2} t^{2}\right)$, three continuous cycle sine signals are used as another modulated signal in Figure 2.5 (b) as reference. The encoding results from these two types of modulated signals will show how the factor exp (-4 $\left.\beta^{2} t^{2}\right)$ works in the binary coded excitation method.

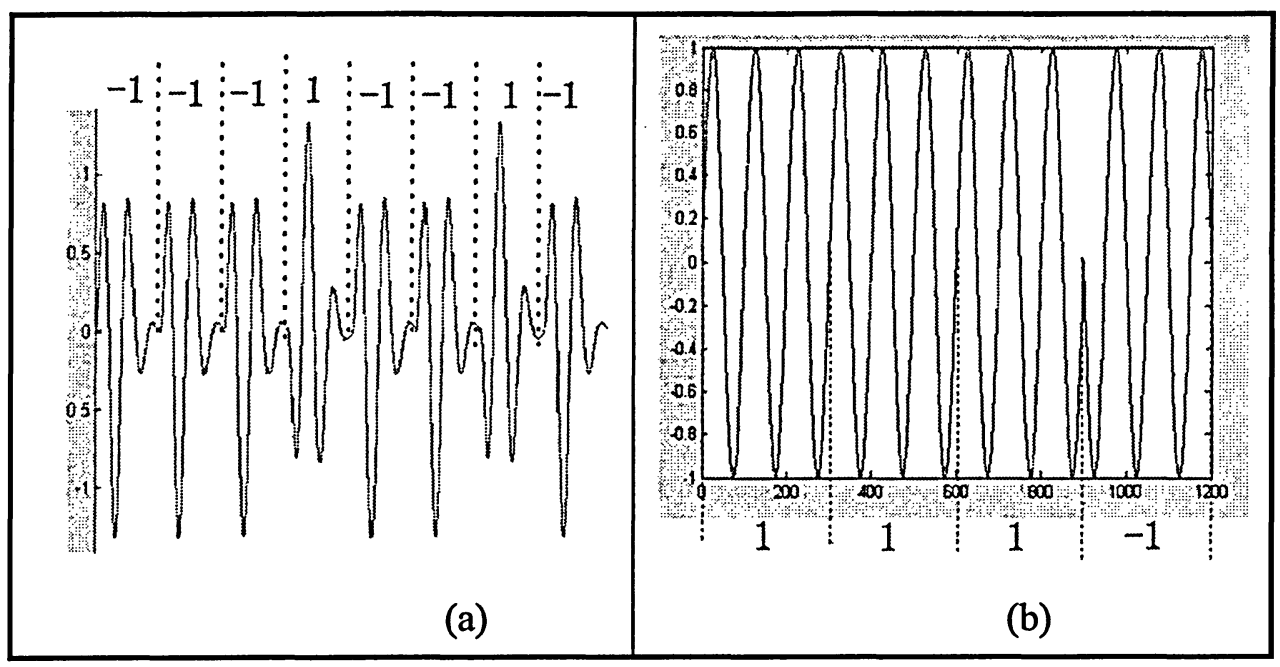

Figure 2.5 (a) The Second Modulated Signals (b) Reference Modulated Signal

Comparing the two signals, Figure (b) uses three-cycle sine per code pattern. In Figure (a) the three cycles are modulated by factor $\exp \left(-4 \beta^{2} t^{2}\right)$. As we expected, the encoding results of Figure (a) have more sidelobes** than Figure (b) due to the signal modulated by the factor $\exp \left(-4 \beta^{2} t^{2}\right)$.

The third modulated signal is shown in Figure 2.6. The transducer requires several

\footnotetext{
** In my thesis, the small reflection pulses due to transducer inertia and small signal from cross correlation are referred as sidelobes.
} 
periods (time $=0.15 \mu \mathrm{s}$ ) to restore. So we sent one cycle of sine signal, then a separation, and second pulse. The real backscatter signal from quartz plate is shown in Figure 2.6 (a). The real reflection signal in Figure 2.6(a) is compared with the signal in Figure 2.4(b). The signal in Figure 2.6 (a) is regarded as three-cycle sine modulated by the factor $\exp \left(-4 \beta^{2} t^{2}\right)$ and some sidelobes mixed in the three-cycle separation between the pulsers. In second method, we also chose three cycles of sine signal and three-cycle separation as modulated signal (shown in Figure 2.6(b)). As we had discussed in the second method, by comparing the encoding results for the signals in Figure 2.6(a) and Figure 2.6(b), we can see how the factors $\exp \left(-4 \beta^{2} t^{2}\right)$ and sidelobes between pulses work in the binary coded excitation method.

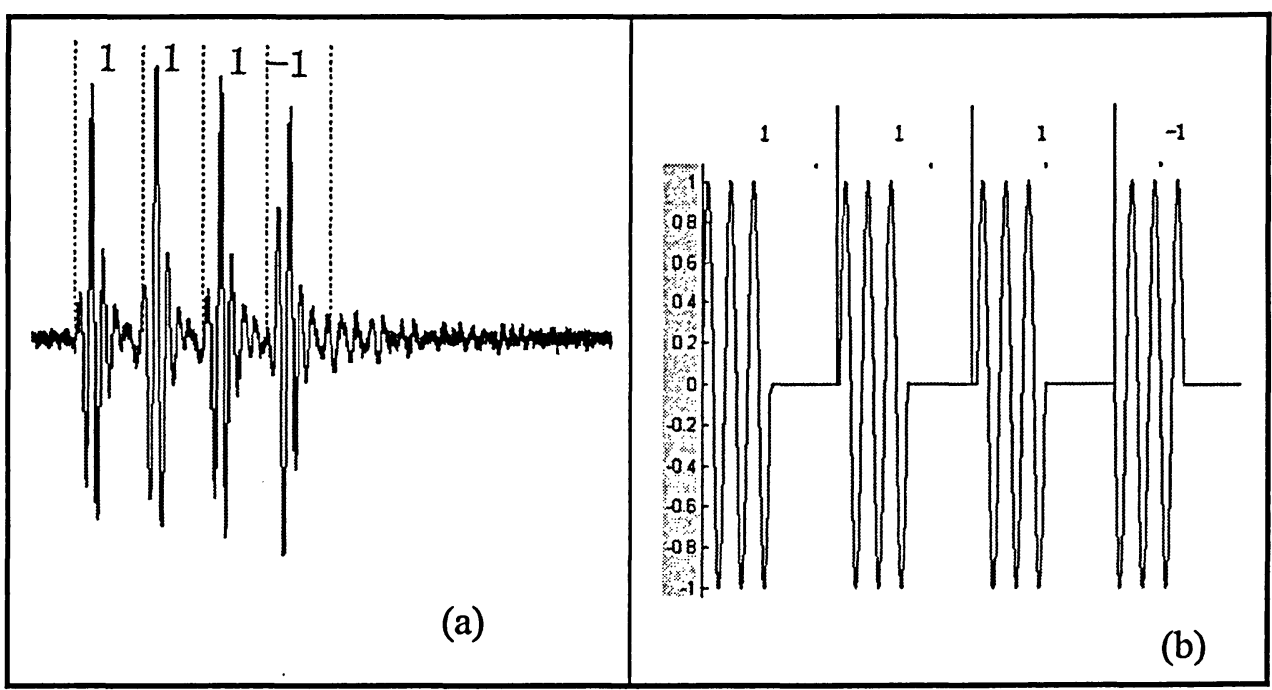

Figure 2.6 Third Type of Modulated Signals

Comparing the two signals, Figure 2.6(b) uses (three-cycle sine and three-cycle separation) per code pattern. In Figure 2.6(a) the three cycle are extra modulated by the factor $\exp \left(-4 \beta^{2} t^{2}\right)$ and some sidelobes noise in the separation. So comparing their encoding results, we can see what the effect of both factor exp ( -4 $\beta^{2} t^{2}$ ) and sidelobes. As we expect, the sidelobes in the separation of pulse increase more sidelobes in encoding results. By comparing the encoding results in Figure 2.6 (a) and Figure 2.5(a), we can figure out how the sidelobes in the separation 
work in the coded excitation method. By simulation of many cases of modulated signals we can see what the effect of special factors, such as sidelobes and exp ( -4 $\left.\beta^{2} t^{2}\right)$, in the binary encoded excitation method.

\subsection{Backscatter Signal}

In this section, we simulate the backscatter signal from tissue. There are three subsections: one dimensional scattering model, attenuation and spacing.

\subsubsection{One Dimensional Scattering Model}

The basic idea of the one-dimensional scattering model has been discussed in Section 1.7.5. The backscatter signal will be calculated using a quasi-periodic one-dimensional model. Backscatter signals are dependent on the scatterer density and scatterer spacing. In order to simplify the backscatter signal calculation, the diffraction, attenuation beam width and focusing effects are ignored.

The quasi-periodic arrangement of scatterers was simulated by distributing scatterers with periodic locations; the spacing distances between scatterers was assumed to be a Gaussian random variable with certain mean and standard deviation. The mean, or the scatterer spacing between scatterers $\Delta z$ was choosen to be 7 to $10 \mu \mathrm{m}$ which is the distance between the normal cells in biological tissue. The membrane and nuclei of cells are believed to be the major contributions to backscatter signal for high frequency ultrasound in biological tissue. Since the scatterers in tissue rarely display perfect periodic arrangement, the standard deviation of spacing is $0.01 \times \Delta \mathrm{z}$.

As shown Figure 1.8, the superposition of echoes from all the scatterers within the 
scaterer region will give us the backscatter. The formulation can be expressed as

$$
s(t)=\sum_{k=1}^{N} a_{k} p\left(t-\frac{2 z_{k}}{c}\right)
$$

$\mathrm{z}_{\mathrm{k}}$ is the location of the $k^{\text {th }}$ scatterer from the origin, $a_{k}$ is the backscattering coefficient of the $k^{\text {th }}$ scatterer, $\mathrm{p}(\mathrm{t})$ is the transmitted pulse, $N$ is the number of scatters present within the range cell, and $c$ is the velocity of sound (assumed 1500 $\mathrm{m} / \mathrm{s}$ ) chosen to be within the range of values measured for soft tissue.

\subsubsection{Attenuation}

In medical ultrasound systems, attenuation reduces the signal amplitude and is frequency dependent The properties of attenuation are discussed in Section 1.6.2. We simulate three different cases of backscatter from tissue: without attenuation, with frequency independent attenuation and with frequency dependent attenuation.

\subsubsection{Frequency Independent Attenuation}

For the case of frequency independent attenuation, we calculate the backscatter using:

$$
p=p_{0} * e^{-\mu E}
$$

where $\mu$ is the attenuation coefficient. The approximate attenuation values for human tissue range between 0.5 to $1 \mathrm{~dB} / \mathrm{MHz} \mathrm{cm}$ (table 1.2). In our simulations we use $0.77 \mathrm{~dB} / \mathrm{MHz} \mathrm{cm}$.

We can convert this to $\mathrm{Np} / \mathrm{MHz} . \mathrm{cm}$ using

$$
\mu_{N p}=0.1151 \mu_{d B}
$$

so the attenuation in $\mathrm{Np}($ Nepers) is $0.088 \mathrm{~Np} / \mathrm{MHz} . \mathrm{cm}$. Therefore, at $40 \mathrm{MHz}$ 
$\mu=3.5 \mathrm{~Np} / \mathrm{cm}=0.35 \mathrm{~Np} / \mathrm{mm}$.

\subsubsection{Frequency dependent attenuation}

The basic idea of frequency dependent attenuation is discussed in Section 1.6.2.2. For most biological tissues the attenuation can be characterized by an attenuation constant $\mu(f)=\mu_{0} f^{n}$, where $\mu_{0}$ is a temperature dependent constant with units that can be expressed as $\mathrm{Np} /\left(\mathrm{cm} \cdot \mathrm{MHz}^{2}\right)$ and $f$ is the frequency and expressed in $\mathrm{MHz}$. $n$ is generally around 1 , but may lie in the range $1 \leq n<2$. We assume $\mathrm{n}=1$; the attenuation coefficient has a liner relation to the frequency of signal. Figure 1.6 demonstrates $n$ for different tissues. The frequency dependent attenuation is

$$
H(f)=e^{-(\mu 0+\mu 1(f-f 0)) z} .
$$

In this formulation attenuation is divided into two parts: frequency independent attenuation and frequency dependent attenuation. $\mu_{0}$ determines the frequency independent attenuation; $\mu_{1}$ determines the frequency dependent attenuation.

Equation (2.4) looks like equation (2.6), however, one is expressed in time domain and the other in frequency domain. Figure 2.7 demonstrates the difference between them.

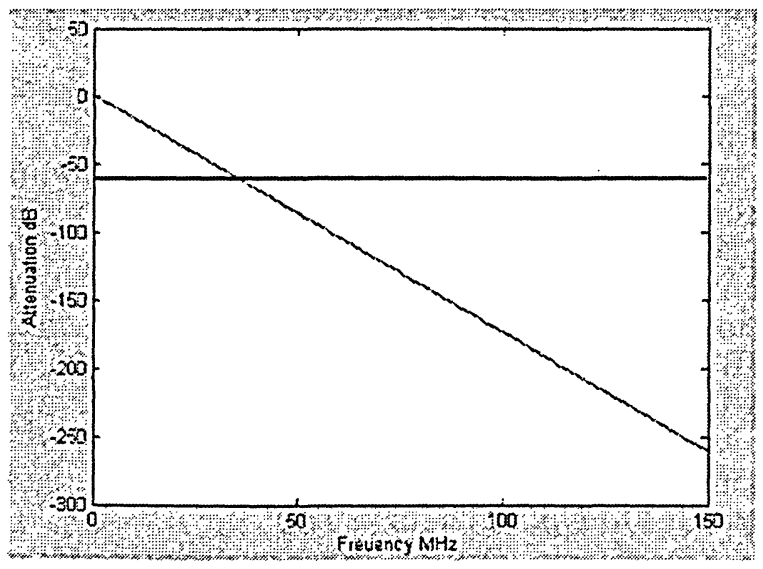

Figure 2.7 Attenuation in the Frequency Domain at $10 \mathrm{~mm}$ 
In Figure 2.7, the $\mathrm{y}$-axis is attenuation in $\mathrm{dB}$ and the $\mathrm{x}$ axis is frequency in $\mathrm{MHz}$. The plain attenuation in the frequency domain is flat. But in the FD attenuation model, the attenuation linearly increases with frequency. The penetration depths for both figures are $10 \mathrm{~mm}$.

In order to calculate the backscatter signals with frequency dependent attenuation the Fourier transforms of the unattenuated backscatter was multiplied by the attenuation transfer function and then an inverse Fourier transform was used to obtain the attenuated waveform.

$$
p(f)=p_{0}(f, z) * H(f, z)=p_{0}(f, z) * e^{-(\mu 0+\mu 1(f-f 0)) z}
$$

The values for $\mu_{0}$ and $\mu_{1}$ were: $0.35 \mathrm{~Np} / \mathrm{mm}$ and $0.0086 \mathrm{~Np} / \mathrm{mm}$, respectively. The procedure is shown in Figure 2.8. For all scatterers, the attenuation factor was recalculated according to their location.

Figure 2.8 demonstrates the steps involved in the implementation of FD attenuation. Figure 2.8 (a) is a coded signal. The signal is expressed in frequency domain in (b). Then a series of FD attenuation coefficients versus frequency (similar to Figure 2.7) are calculated. We multiply the signals in frequency domain by their FD attenuation coefficients based on their corresponding frequency range. From Figure 2.8 (a) and (e), we can see the signal in high frequency attenuates more than the low frequency part. 


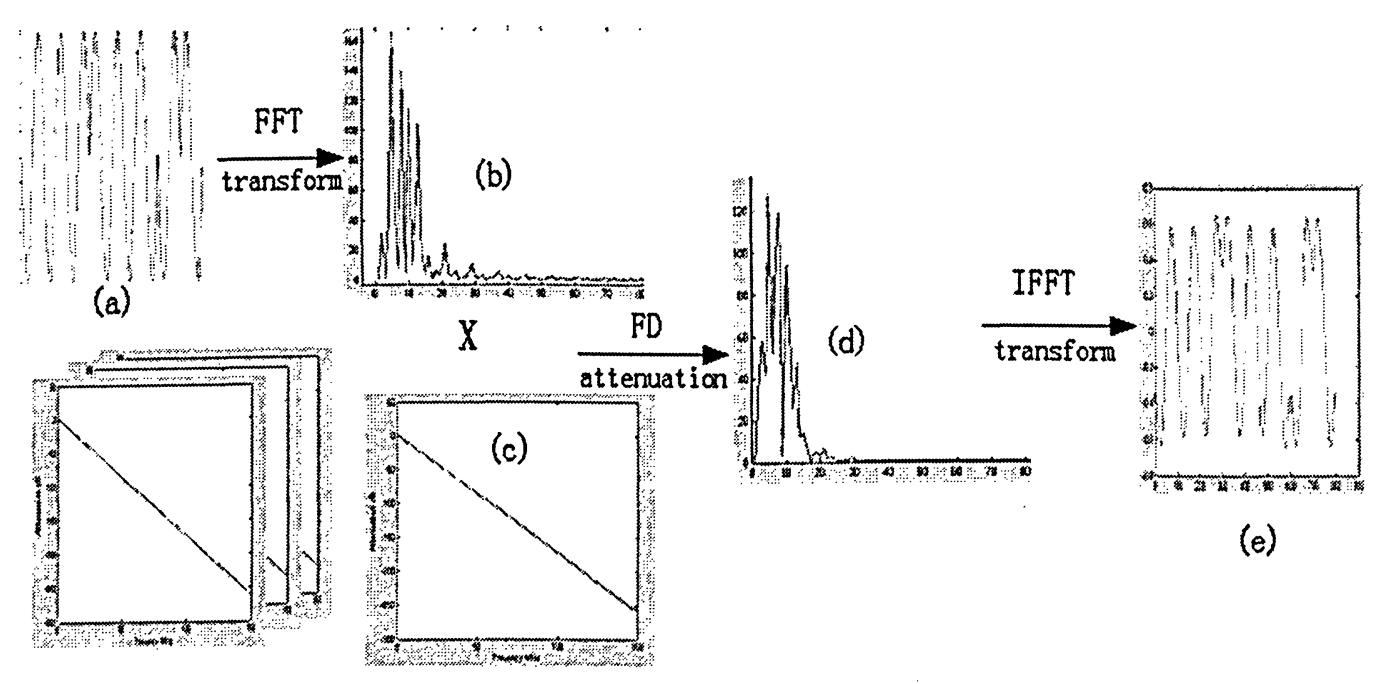

Figure 2.8 Implementation of FD Attenuation.

In the Figures (b), (c) and (d) the $\mathrm{x}$-axis is the frequency in MHz; In Figure (b) and (d) $y$ axis is the amplitude of signal in frequency domain. In Figure (c) y-axis is the attenuation in dB. In both Figure (a) and (e) the $x$-axis is the time and $y$-axis is the amplitude of signal.

\subsubsection{Spacing}

In our simulations, we consider two different sets of spacing: sparse and dense spacing. In sparse spacing the distances between two adjacent scatterers are large enough to identify each backscatter. In dense spacing the distances between two adjacent scatterers are about the diameter of cells (7 to $10 \mu \mathrm{m}$ ).

The sparse spacing model was used to analyze if our implementations were correct or not and to examine the effect of FD attenuation on the incident pulse. In each simulation the sparse spacing was implemented first. Then, if the result was correct, we used dense spacing in the program to simulate tissue backscatter. For example, from Figure 2.9 (b) it not easy to verify if the signal is implemented 
correctly, but in Figure 2.9 (a) we can identify the correct signal by zooming inio the signal.

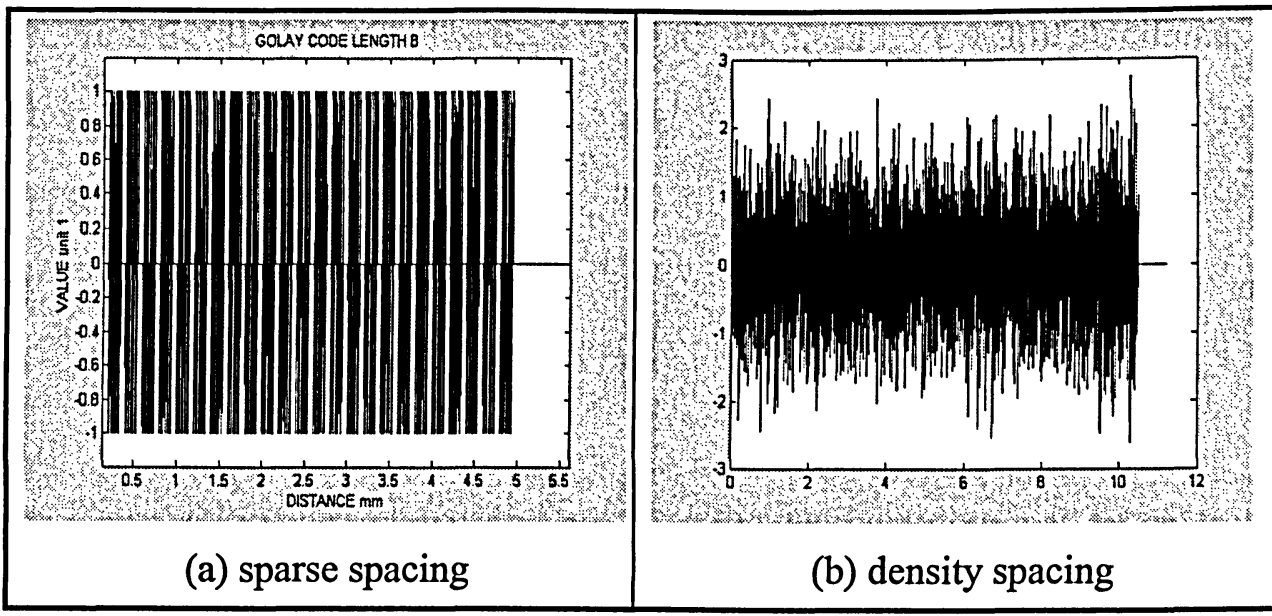

Figure 2.9 Simulation Results with Different Spacing

In both figures $\mathrm{y}$-axis is the amplitude of signals and the $\mathrm{x}$-axis is the penetration depth in $\mathrm{mm}$.

\subsubsection{Processing Coded Backscatter Signals}

In Section 1.10.2, a code sequence itself is chosen as a processing reference signal to cross correlate to the received signals for the coded excitation method. The next few sections deal with the proper choice of the cross-correlation reference. Three different reference signals are introduced to process the coded excited signals in this section. They are reflection reference processing method, code reference processing method and code with phase reference processing method.

\subsubsection{Reflection Reference Processing Method}

In the first method the reflected signal from a quartz plate is chosen as a reference signal. When studying the backscatter signal from a quartz reflection, the 
processing reference signal is the same as the signal. In Figure 2.10 (a) one quartz reflection for code length 2 is shown and in (b) is the processing reference. When the signal in Figure 2.10(a) is truncated and then normalized its maximal amplitude to 1, we get its processing reference as Figure 2.10 (b). Before sampling real backscatter signals for a coded sequence, we should save the quartz reflection of this coded sequence to use as a cross correlation reference. This method is referred to as the reflection reference processing method and is equivalent to processing the signal as a matching filter.

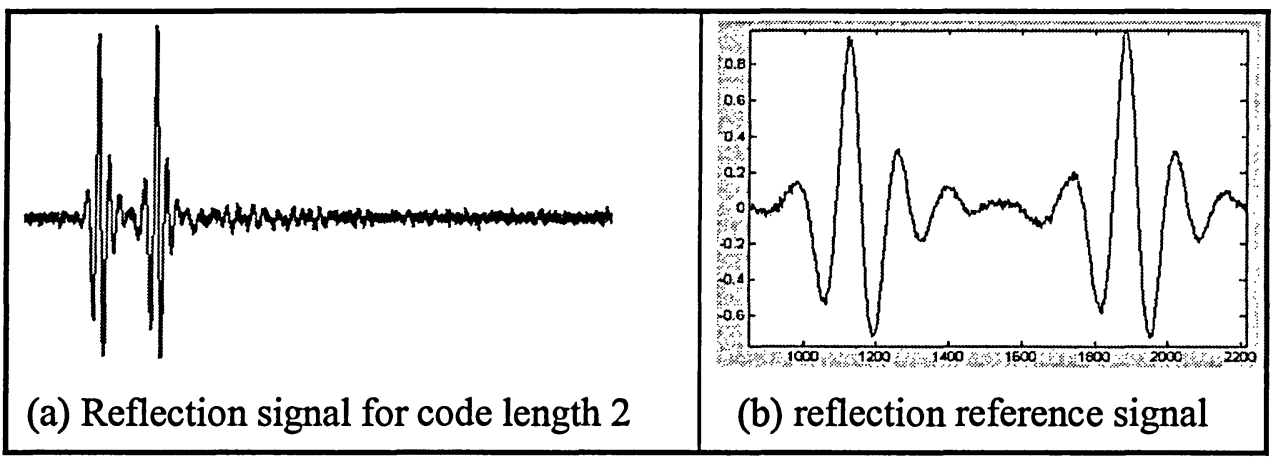

Figure 2.10 Reflection Reference Processing Method

\subsubsection{Code Reference Processing Method}

In most simulations we used the code reference processing method. The basic idea in this method is that Golay codes are used as processing reference. For example, when the code is 1 and -1 , we use one cycle sine per code-modulation. The encoded signal sent to the transducer is shown in figure 2.11(a) (dot one). For the bit of 1 in code we use one cycle sine pulse; one cycle inverse sine is used to represent the bit of -1 in code. The backscatter signal from quartz reflection is the solid line in Figure 2.11(a). We can consider as our cross-correlation reference, the thick dot dash line in figure 2.11(a). To be consistent with the excitation pulse, we extend the 1 bit Golay code to $25 \mathrm{~ns}$ (one period of $40 \mathrm{MHz}$ ). 
The backscatter signal from the quartz plate, however, has three cycles of signal. We can extend the length of code to 3 cycles to achieve higher gain. And generate a new processing reference. The reference is the thick dot dash line one in Figure 2.11(b). Since both references are directly derived from the code 1 and -1 , both methods are called code reference processing methods. In order to identify these two methods, the first one in Figure 2.11(a) is called the one-cycle code reference and the second one in Figure 2.11(b) is called the three-cycle code reference.

The three-cycle code reference processing method produced the worst results compared with the others, because it mixes all signals together. The one-cycle code reference processing method has the interesting property that its encoding result has the same shape as the reflection from one pulse excitation as shown in Figure 2.12(b). Therefore the axial resolution will be similar to the conventional one pulse excitation. Later in chapter 3 we will see the encoding results from other processing methods consisting of 6 cycles instead of 3 . So the axial resolution for all other processing methods degrades by half compared to the one-cycle code reference processing method, but these methods provide high encoding gain.

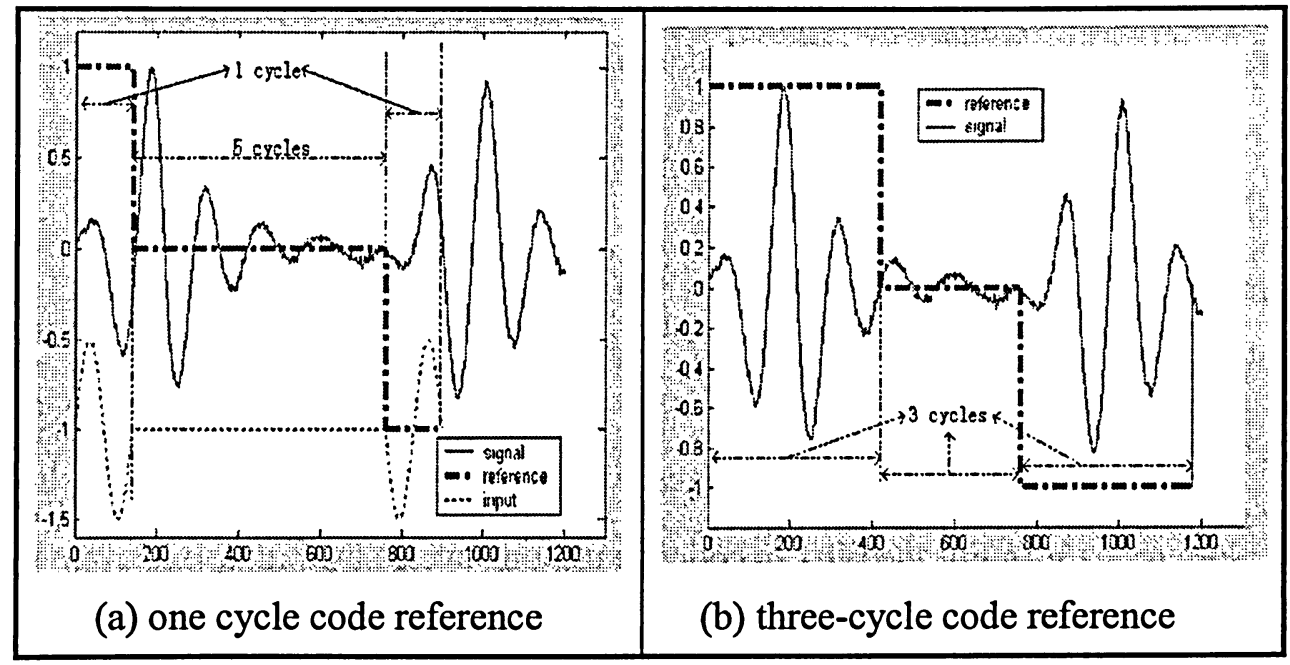

Figure 2.11 Code Reference Processing Method 
In Figure 2.11, the $y$-axis is the signal amplitude and the $\mathrm{x}$-axis is sampling points. The sampling rate is $5 \mathrm{GHz}$ so the period of each cycle is about $25 \mathrm{~ns}$.

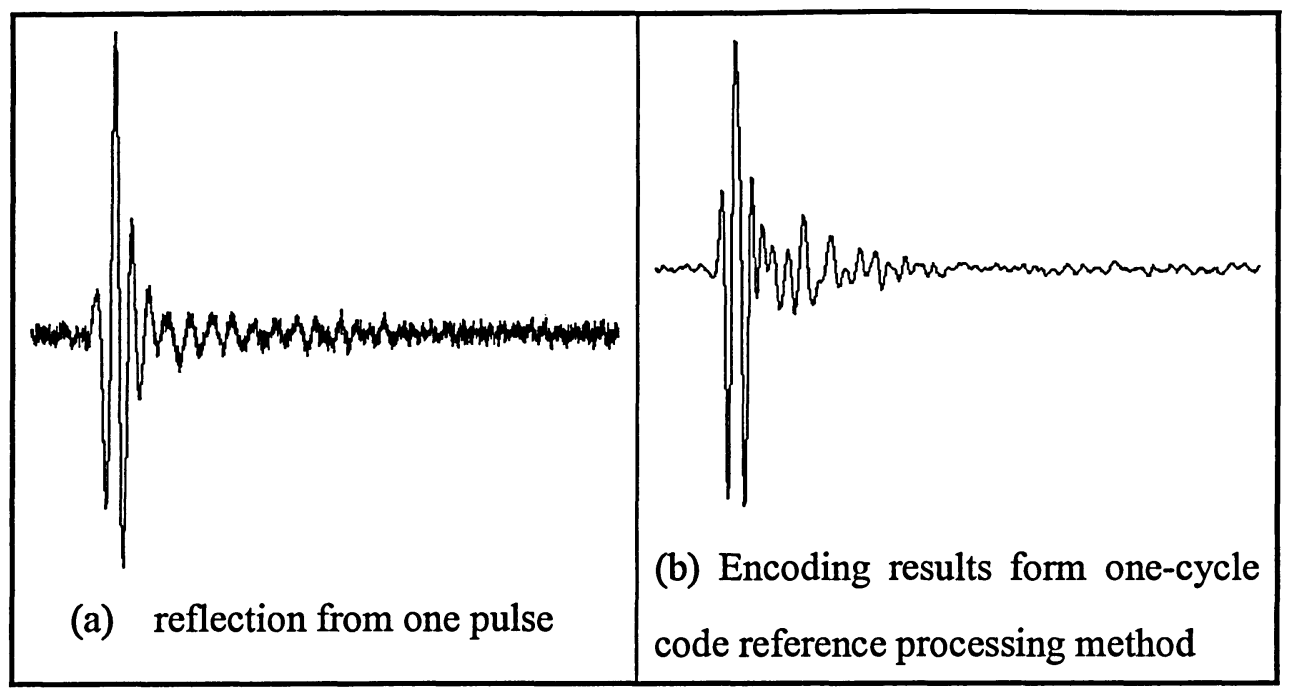

Figure 2.12 Results from One Cycle Code Reference and Reflection from One Pulse

This Figure (a) is the quartz reflection of one pulser input, (b) is the encoding results from quartz reflection of code length 2 processing by one cycle code reference processing method. Both signals have the same number of large pulses.

\subsubsection{Code with Phase Reference Processing Method}

The third method is referred to as the code with phase reference processing method. This method also uses the code as the cross correlation reference as the second one does. The encoded signal is sent into transducer as dot line shown in Figure 2.13(a). For the bit 1 in code we use one cycle sine pulse; one cycle inverse sine is used to represent the bit of -1 in code. The backscatter signal from quartz reflection is blue solid one in Figure 2.13(a). 
The only difference is that the signal phase is considered this time. The reference we still use is 1 and -1 . But we divide one cycle input sine ( $25 \mathrm{~ns})$ into two parts in Figure 2.13 (a). When the encode sine signal is half positive one, we use 1 for half cycle $12.5 \mathrm{~ns}$ and -1 for $12.5 \mathrm{~ns}$ for the negative half cycle of sine. The thick dot dash line is cross correlation reference in Figure 2.13 (a). In order to accumulate all the energy for the 3 cycles we extend the code with phase to 3 cycles as we did for the code reference method.

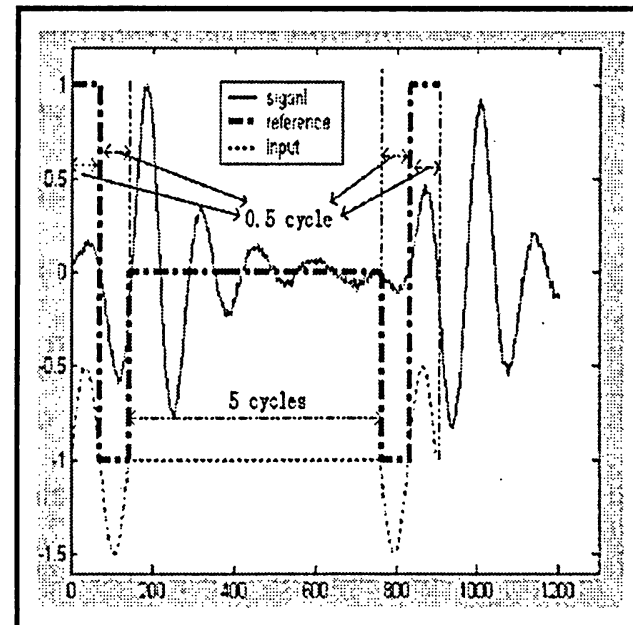

(a) One Period Reference

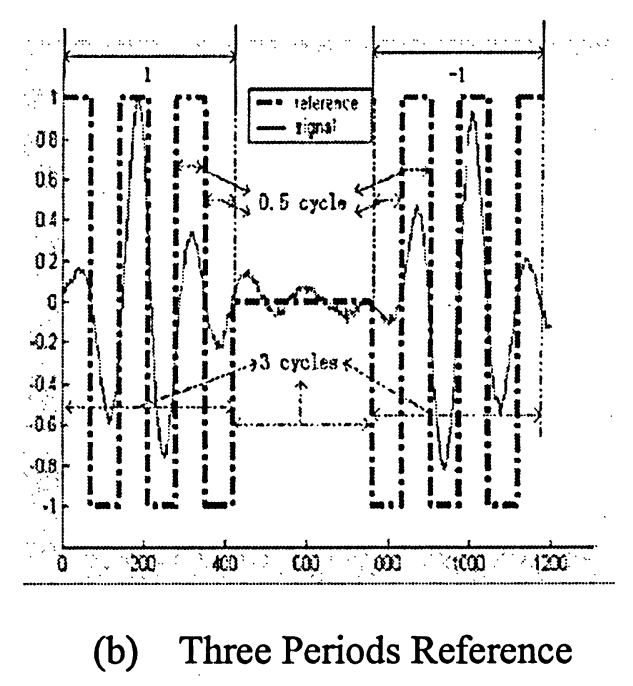

Figure 2.13 Code with Phase Reference for Cross Correlation

In Figure 2.13, the $y$-axis represents the signals and the $x$-axis the sampling points. (the sampling rate is $5 \mathrm{GHz}$ so the period of each cycle is about $25 \mathrm{~ns}$ )

Both figures in Figure 2.13 demonstrate the code with phase reference processing methods. The method shown in Figure 2.13 (a) is the one-cycle code with phase reference method and that in Figure 2.13 (b) is the three-cycle code with phase reference method. This time the three-cycle code phase reference almost doubles the gain than the one cycle code phase reference. 


\subsubsection{Simulations}

A schematic of how the computer program I developed in MATLAB is shown in Figure 2.14. Input parameters are the time interval, the number of scatterers, the center frequency, the attenuation coefficient and the spacing $\Delta z$. Then Golay code and complimentary code are used to modulate the signal to calculate the signal to be sent out. The third step uses a random function generator to generate the deviation of the distance between scatterers. The fourth step is to calculate the time shift for different points. In the fifth step, the pulse signal is convolved with each scatterer (equation 2.3). In the models of without attenuation and with frequency independent attenuation, we just multiply the result with an attenuation factor; the procedure of frequency dependent model is discussed in section 2.3.2.2. We add all calculations for each scatterer to form the received signal. In order to find out encoding results for the coded sequence, we can cross correlate the backscatter signals from Golay code with its reference and complimentary code with its reference separately and finally add these two cross correlation results together to obtain the encoding results. 


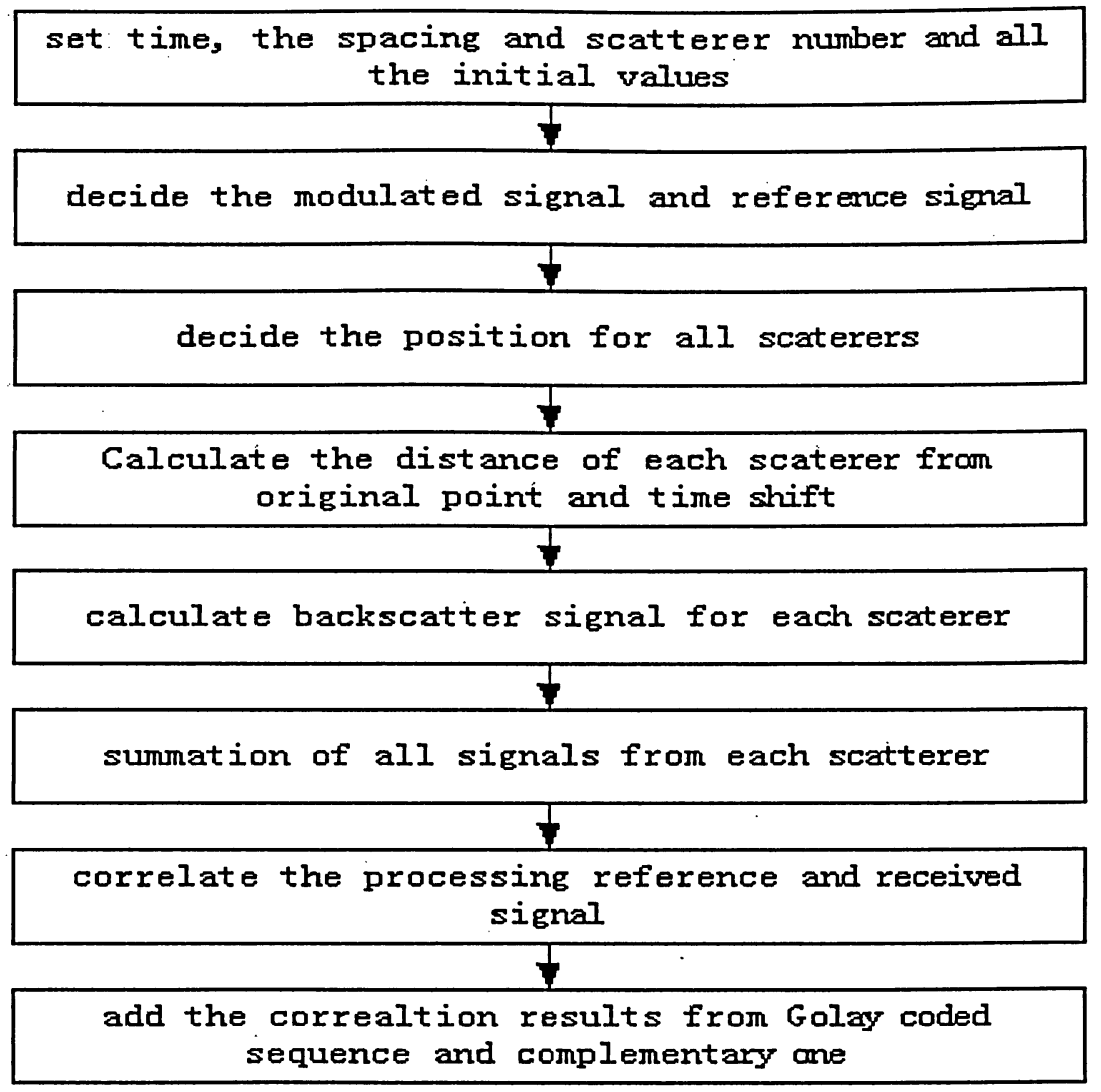

Figure 2.14 Schematic diagram of the Program

\subsubsection{Evaluation Methods}

As the purpose of this investigation is to evaluate methods that would increase the signal to noise ratio in high frequency ultrasound, signals with and without pulse encoding must be compared. Several methods that can be used are described as follows.

The simplest way to evaluate the signal gain is by comparing the signal amplitudes. The autocorrelation of codes can be used to analyze the results of a sparse spacing simulation and quartz reflection from our experiments. Another way is by exerting the Signal to noise ratio. 


\subsubsection{Signal to Noise Ratio}

This method is very important to evaluate the coded excitation method. Only the increase in signal to noise ratio can deepen penetration depth. For our system, noise comes from multiple sources including the transducer and preamplifier. The received signal strength due to a scattererer at a given depth will depend on the incident signal strength, the attenuation, and the backscatter coefficient. For the signal will be weaker with depth, the signal to noise ratio is depth dependent and can be defined by:

$$
\mathrm{SNR}=\frac{\text { the received }}{\text { signal power }}
$$

In ultrasound systems, generally two types of noise needed to be discussed. They are random noise and speckle noise. Random noises such as the receiver thermal noise and the amplifier thermal noise are white noise and the white noise is uncorrelated. But the speckle noise is not white noise. It comes from interference of waves backscattered from many closely spaced scatterers (spacing much smaller than the compressed pulse width). Speckle noise results from a convolution process and is uncorrelated.

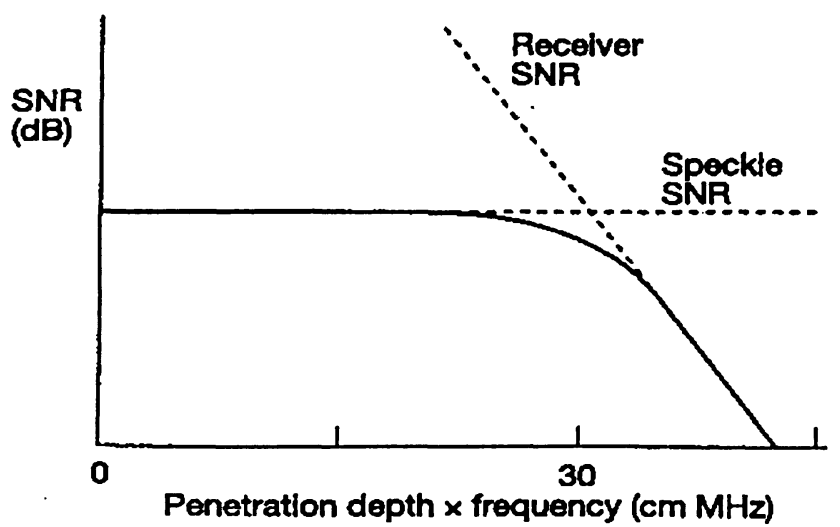

Figure 2.15 Noise Type and Penetration Depth $\times$ Frequency 
How to consider the white noise or speckle noise in an ultrasound system? As Figure 2.15 shown, Hill give a relation on the noise type to penetration depth $\times$ frequency by studying the concept of contrast resolution. From this figure, at lower frequency and shallower depth, the speckle will make major contribution to total noise; at higher frequency and deeper depth, the receiver thermal and other white noise will be dominant in SNR.

But sometimes the speckle is not noise; we are interested in speckle pattern. The speckle signals have some information of the structure of tissue and it is this information that we are interested in for our application. For example, all of the signals in Figure 1.9 are speckle. From Section 1.7.2 we know that scattering contributes more in high frequency range to the attenuation coefficient, so speckle in high frequency is stronger than that in low frequency.

Since we study high frequency ultrasound system $(40 \mathrm{MHz})$, and we are mainly interested in the structure patterns, the speckle noise will be neglected. We assume the noise from transducer and preamplifier are white over the bandwidth of system $\Delta f$. If the noise power density is $N n$, the total energy of noise is expressed as $N n \times$ $\Delta f$. If the received signal is sinusoidal pulse of duration $\mathrm{T}$ and amplitude $\mathrm{A}$ is considered, the energy of received signal can be expressed as:

$$
\mathrm{E}=\frac{1}{2} A^{2} T
$$

So that:

$$
\mathrm{SNR}=\frac{A^{2}}{N n^{*} \Delta f}=\frac{2 E}{T^{*} N n^{*} \Delta f}
$$

If the optimum reception filter is used the bandwidth $\Delta f=1 / \mathrm{T}$, the formulation can be change into:

$$
\mathrm{SNR}=\frac{2 E}{N n}
$$

From Equation (2.14) if we want to increase the SNR, we should increase the amplitude of signal and decrease the noise level. Increasing signal amplitude 
directly is limited by the safety concerns and nonlinear propagation distortion. So in binary coded excitation method the duration of signal is increased to increase the total energy to be received.

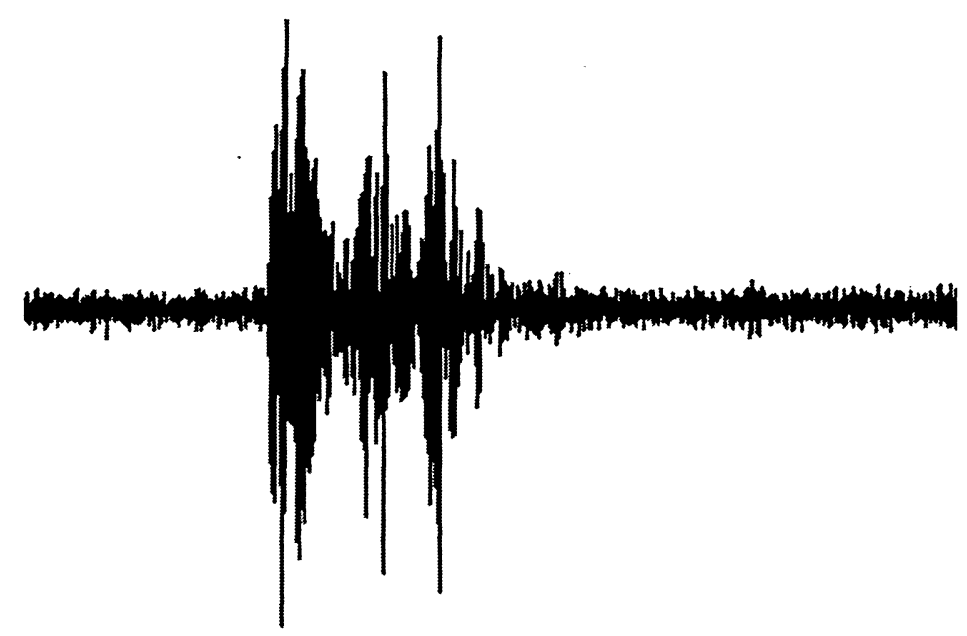

Figure 2.16 A Real Backscatter Signal from Bovine Liver

How can we estimate the signal and noise power? If we can answer these two questions, then the SNR can be evaluated. The energy of signals can be expressed, as the mean of backscatter signal is almost zero, the STD (standard deviation) of the signal represents the power of the signal. For noise we can analyze Figure 2.16, a backscatter signal from bovine liver. From this figure we can see there are noises on both sides of backscatter signal. The STD of the noise amplitude can be used before cross correlation is used to estimate the power of noise.

How can we estimate the noise after the correlation operation? Assume the noise appears as independent random samples with variation $\sigma^{2}$. The resulting noise in the final estimation is likewise consists of independent samples $(N$ the number of samples), with variation $N \times \sigma^{2}$. The reason is because the cross-correlation adds $N$ independent samples for each output point. The amplitude of the noise in output is $\sqrt{N}$ times that of the noise before cross correlation. 


\subsubsection{Signal Distortion}

The ideal system is such a system that the output of system is the same as the input of system. There are some distortions in real system. A lot of factors cause the signal distortion. These factors include frequency dependent attenuation, inertia of transducer, accidental phase shifts during data acquisition, focus change and so on. We have to find a way to evaluate the signal distortion.

The correlation coefficient can be used to evaluate the similarity of two signals. This method is often used in radar and sonar applications. The received signal reflected from the target is the delayed version of the transmitted signal, so the correlation can be used to evaluate the distortion of the signals.

\subsubsection{Signal Envelope}

When one cycle of sine signal is sent out from the signal generator, the received signal from the reflector is shown in Figure 2.4(a). It is difficult to compare this signal to the sine pulse.

The envelope of the signal from a point scatterer or reflector will be the same as the incident envelope provided there are no frequency dependent effects. It should provide an estimate of the envelope that closely matches the transmitted envelope. As presented in Figure 2.17, it is straightforward to determine the envelope. If $x(t)$ is a real received signal, then its Hilbert transform has a frequency spectrum identical to that of the input. Note that the phase components are all shifted by $-90^{\circ}$, i.e., the Hilbert transform acts as an ideal $-90^{\circ}$ phase shifter. As shown in (b), for a Gaussian received pulse, the envelope will be Gaussian and the Hilbert transformed output will be quasi-sinusoidal with a similar envelope. 


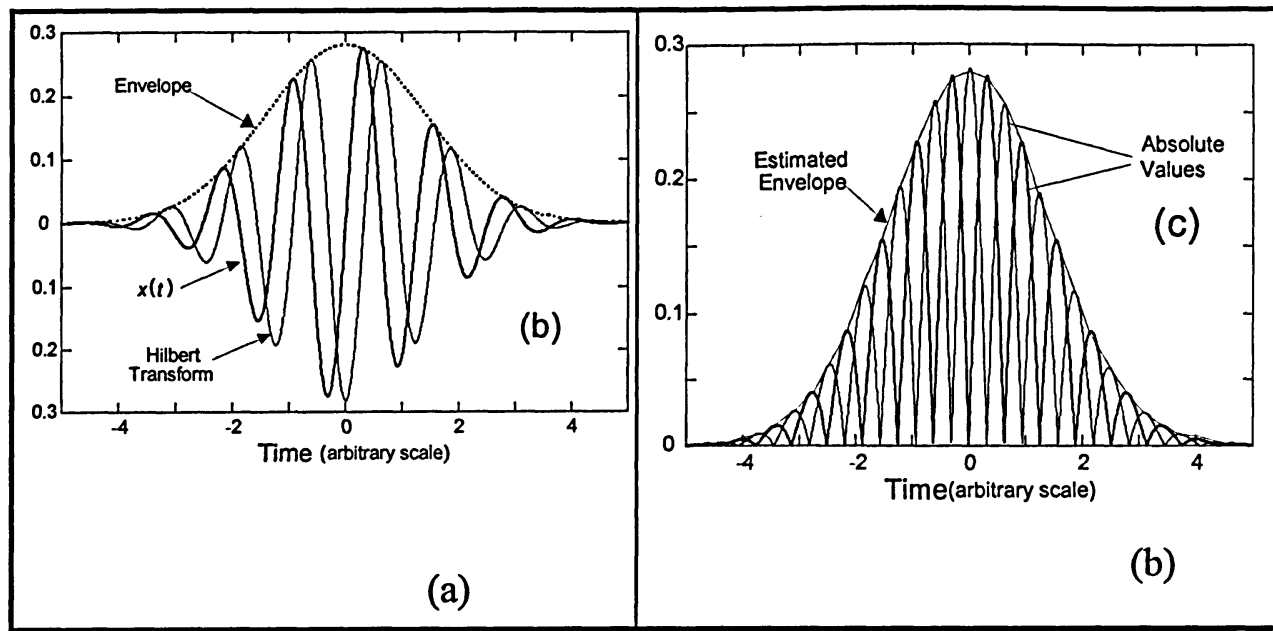

Figure 2.17 Getting Envelop of Signal by Hilbert Transform ${ }^{[2]}$

\subsection{Experimental Prototype of Binary Coded Excitation System}

In Section 1.9 and Section 1.10.5, the structure of ultrasound system and binary coded excitation system are discussed. Combining Figures 1.13 and 1.25, the structure of the binary coded excitation ultrasound system is shown in Figure 2.18.

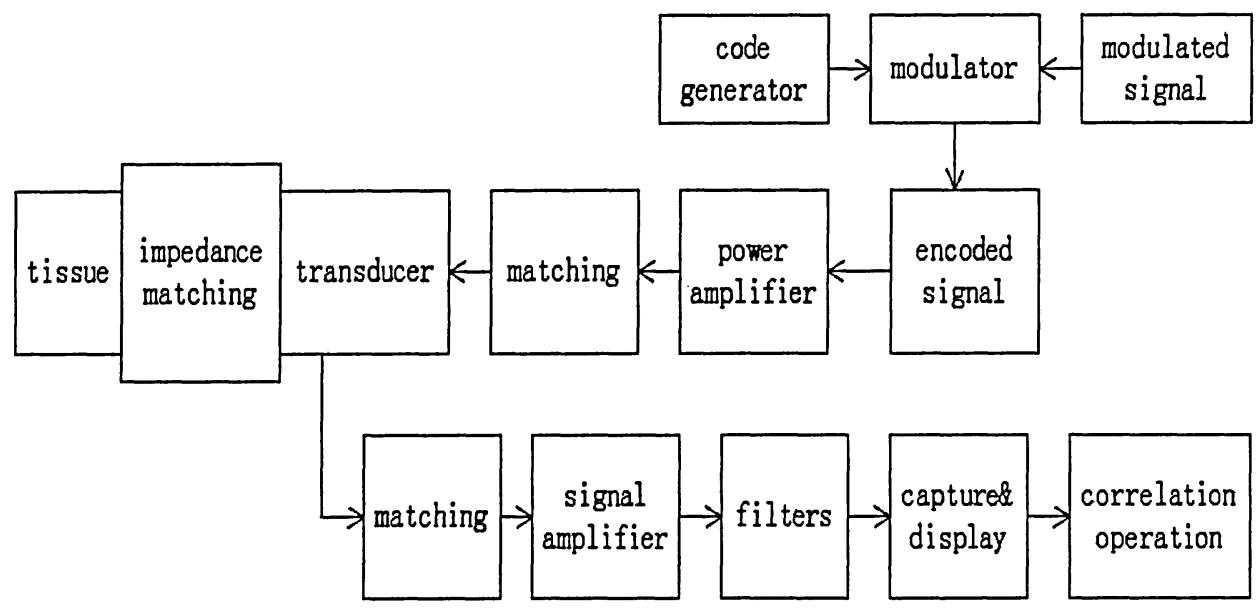

Figure 2.18 The Structure of Binary Coded Excitation

In order to construct such a system, several questions such as how to choose the 
signal generator, the code generator, the modulator, the power amplifier, the signal amplifier, how to design the filter, how to design matching circuit, how to sample the received signal and how to process the received signal, must be answered first.

In our project, a binary Golay coded excitation ultrasound system was successfully designed. I selected the first group of Golay code pairs from Section 2.2.1.2. The modulated signal we used is a cycle of a sine signal. This one cycle sine signal was modulated with different Golay code pairs. An arbitrary waveform generator was used to complete this task. The functions of code generator, the modulated signal generator and modulator are implemented in the arbitrary waveform generator. The Golay code encoded signal is amplified and then sent to the transducer.

How are the received signals processed? In my experiment, we sampled transducer-received signal and saved them in files. Post-processing of the backscatter data were completed on PC. Therefore, no special hardware to implement the cross correlation operation was required.

\subsubsection{The Experimental System}

In Figure 2.19, the left blue instrument is the arbitrary signal generator; the middle white instrument is the power amplifier; the right one is the oscilloscope; there is a lab platform between the big amplifier and the oscilloscope; a transducer, signal amplifier and filters on the support of platform. Next we will discuss each part of the lab system. 


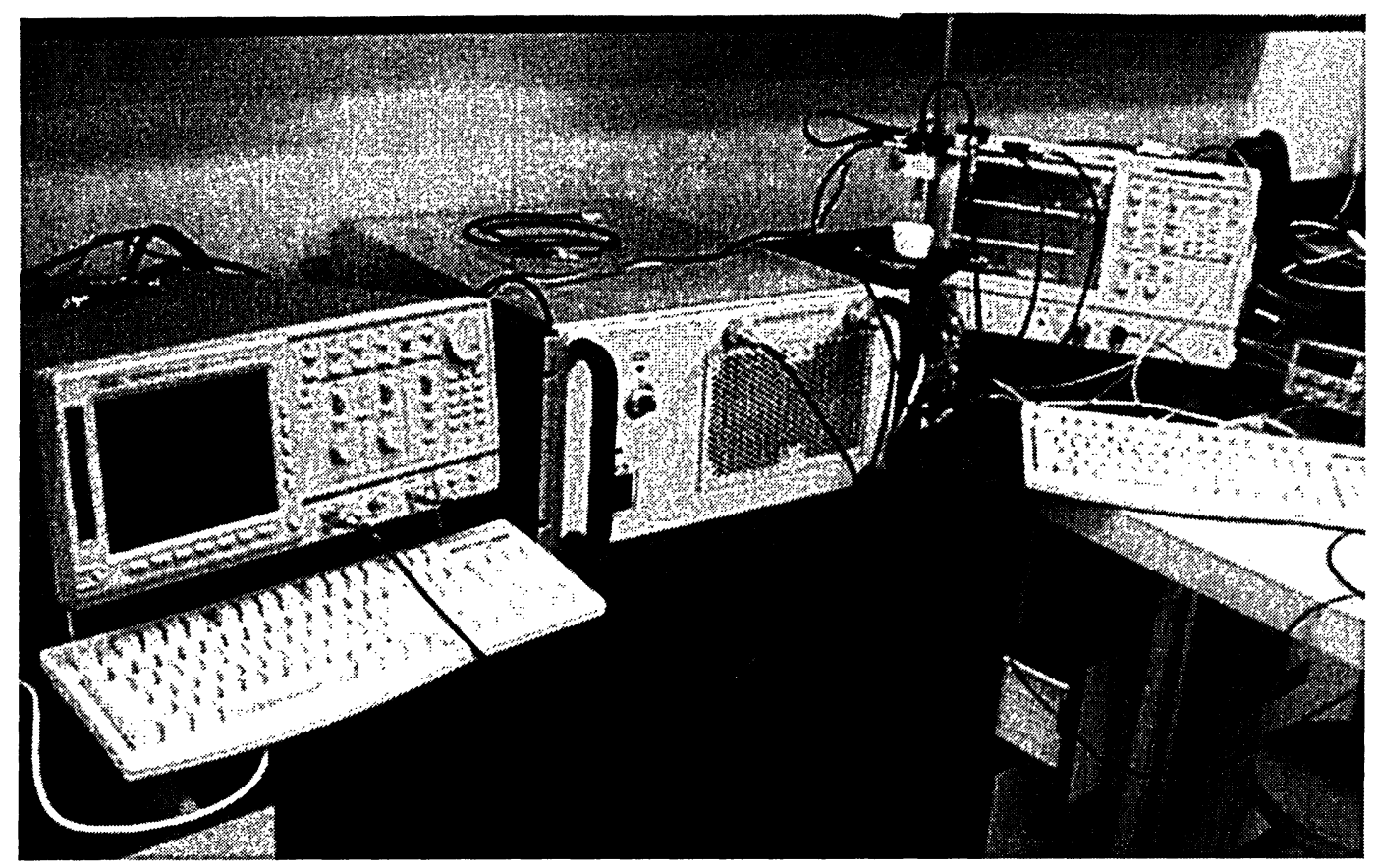

Figure 2.19 Prototype Binary Coded Excitation System

I constructed the experimental system by myself. In the Appendix A, all the parameters of our instruments are listed. How to match electrical parameters of all the instruments of the system; how to generate coded signals by arbitrary waveform generator; how to design a low pass filter, a high pass filter and an expander are discussed in detail in this Appendix. The PCB layout and PSPICE simulation of our circuits are listed also.

After the lab system was set up, we took several months to adjust our experimental system. We successfully suppressed the noise problem as explained in Appendix B.

\subsubsection{Two Interesting Phenomena}

\subsubsection{Transducer Focus}

In common sense that the focus of a transducer is decided by the material, structure and shape of the transducer and varies with properties of surrounding 
material in which the transducer is used. It is assumed that the focus of transducer has no relationship to the signal that is sent to the transducer and is primarily determined by the transducer geometry (Figure 1.4). However, we find that when we increased the code length from 1 to 16 , the focus of the transducer changed, as measured by the location at which a quartz reflector was placed to receive the greatest backscatter signal.

In our experiment, the transducer focus, when the code length was 1 , was measured and fixed. Then the input signal was changed to a Golay coded sequence, code length 16. The reflection signal is shown in Figure 2.20. The time in the figure is from reference index to time index. This time is the time between the excitation pulse and receipt of the signal from the transducer, so the time also represents the distance that the signal travels. The amplitude of the first reflection is about $200 \mathrm{mV}$. We kept the input signal unchanged and increased the distance (about $1 \mu \mathrm{s}$ in time) from the transducer to the reflector. The amplitude of the quartz reflection increased (Figure 2.21). Specifically, the first several pulses decreased from $320 \mathrm{mV}$ (Figure 2.21) to $200 \mathrm{mV}$ (Figure 2.20). At the new position we get more energy than that at the old position, where is the transducer focus for one pulse. Therefore, we conclude that the transducer focus changes with code length. We are not sure why.

If you see the signal of reflection, we find it is cone shaped. When we use the encoded signals of code length 2 and code length 4, the reflection signals are uniform and the amplitude is near $1 \mathrm{~V}$; while the maximal values in Figures 2.20 and 2.21 are just $550 \mathrm{mV}$. This extra attenuation means that the encoded gains for code length 8 and 16 are not as large as our simulation. 


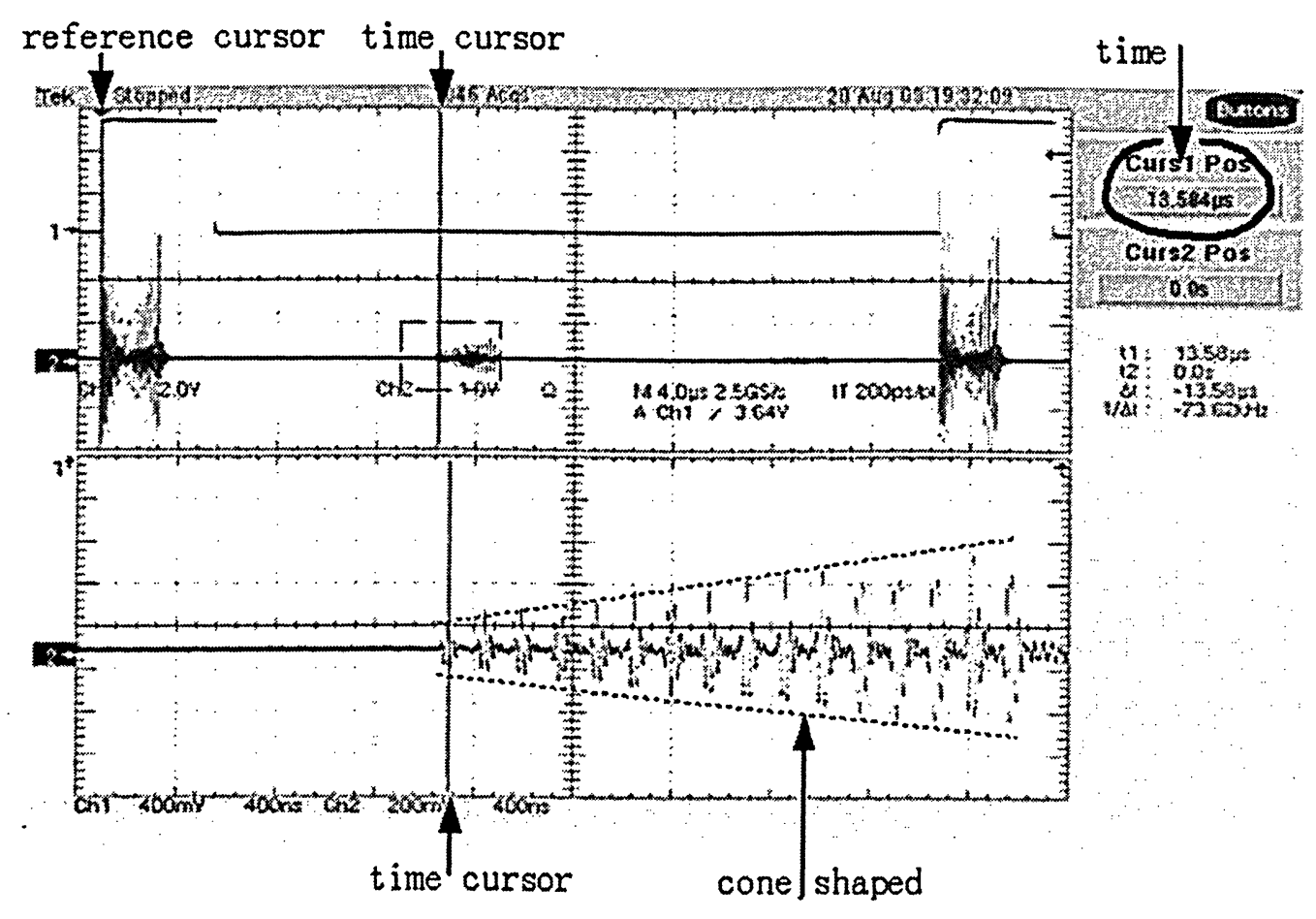

Figure 2.20 Reflection Signal for Code Length 16

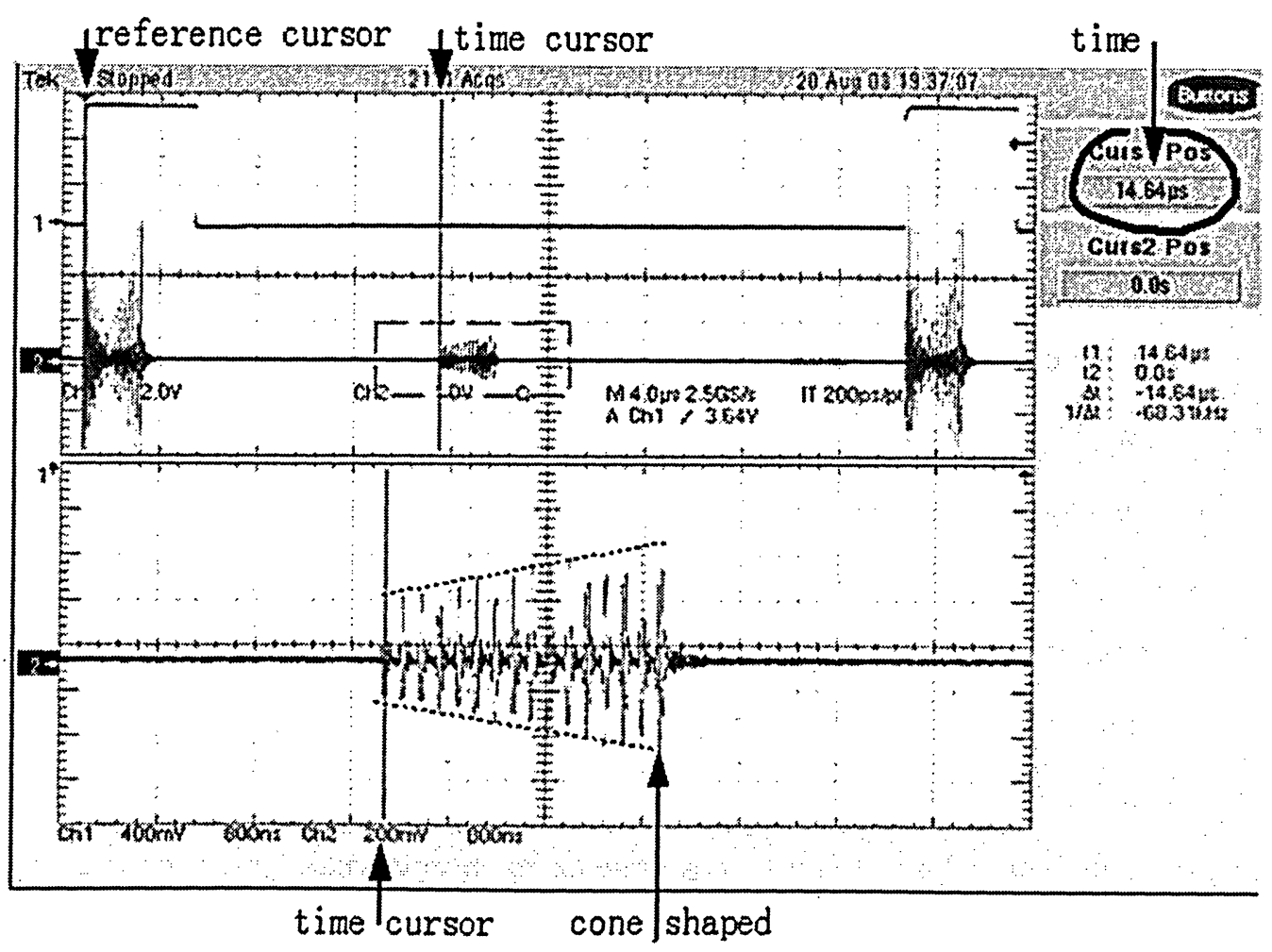

Figure 2.21 Focus Point for Reflection Signal of Code Length 16 


\subsubsection{Phase Shift}

In the experimental prototype system, Golay code and complimentary Golay code pairs cannot be sent to the transducer at the same time. Both signals are sent in a certain sequence, as shown in Figure2.22, the received signals have a small moving phase shift. If the both Golay and complementary Golay are sent out simultaneously, the moving shift can be minimized and the moving phase shift has less blur effect on this binary coded excitation method. So it is not necessary to consider phase compensation. The solid and dot lines denote the quartz reflections of the Golay code and the complementary Golay code (code length 4). The moving distance between the two reflections is about $17 \mathrm{~ns}(20$ to $30 \mu \mathrm{m})$.

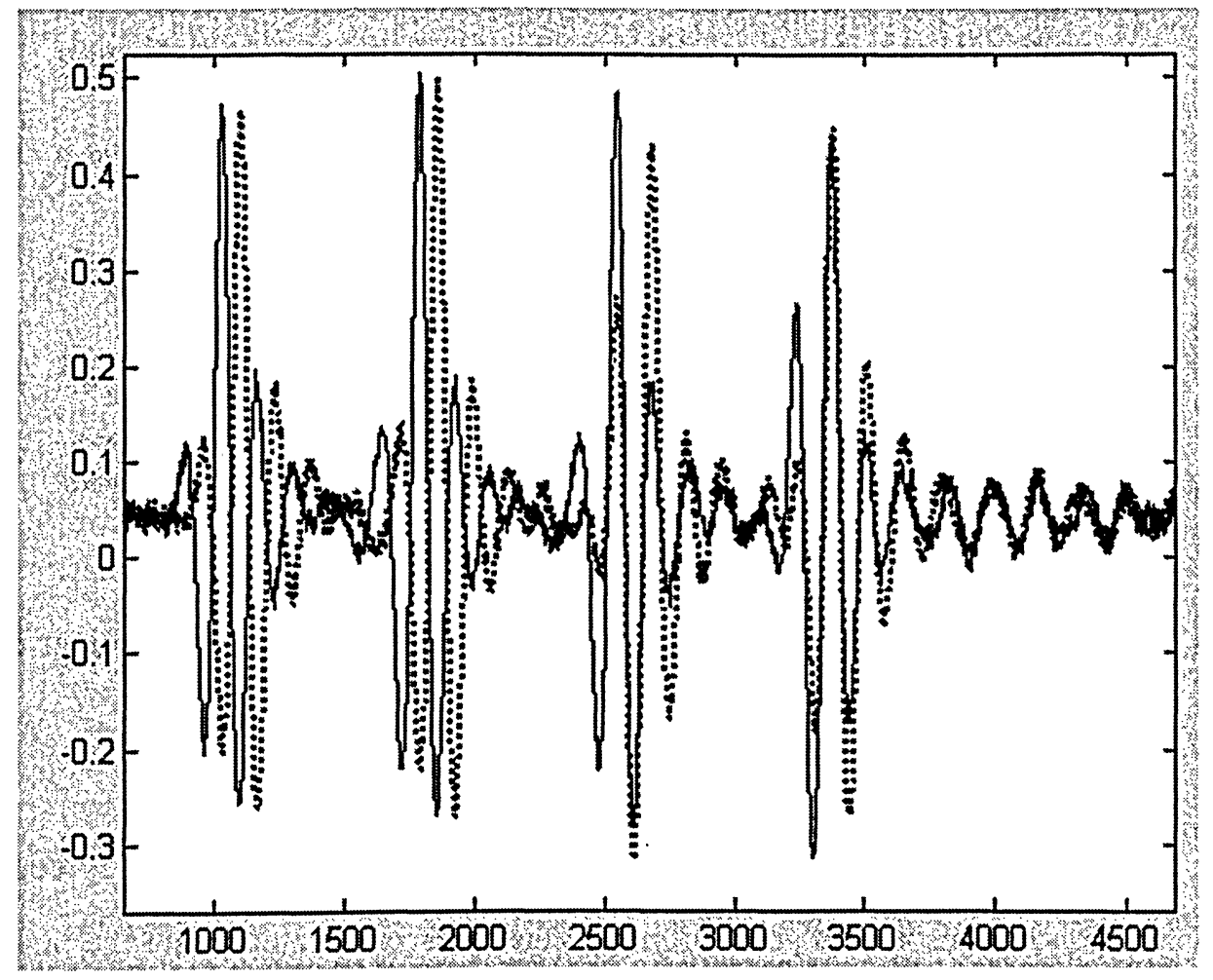

Figure 2.22 Phase Shift Between Golay Pairs $[1,1,1,-1]$ and $[1,1,-1,1]$

After we put some supporting materials to stabilize the platform, the moving phase shift between the Golay and complimentary Golay pairs disappears (as 
shown in Figure 2.23). The solid and dot lines denote the quartz reflections of the Golay code and the complementary Golay code (code length 4). In this figure, both signals are not processed with manual compensation. Therefore, we assume the movement of the platform causes the phase shift. Since it is possible that our target tissues are movable, the phase shift has to be taken into account for binary coded excitation method. The phase shift from our platform can be solved by a careful stabilization.

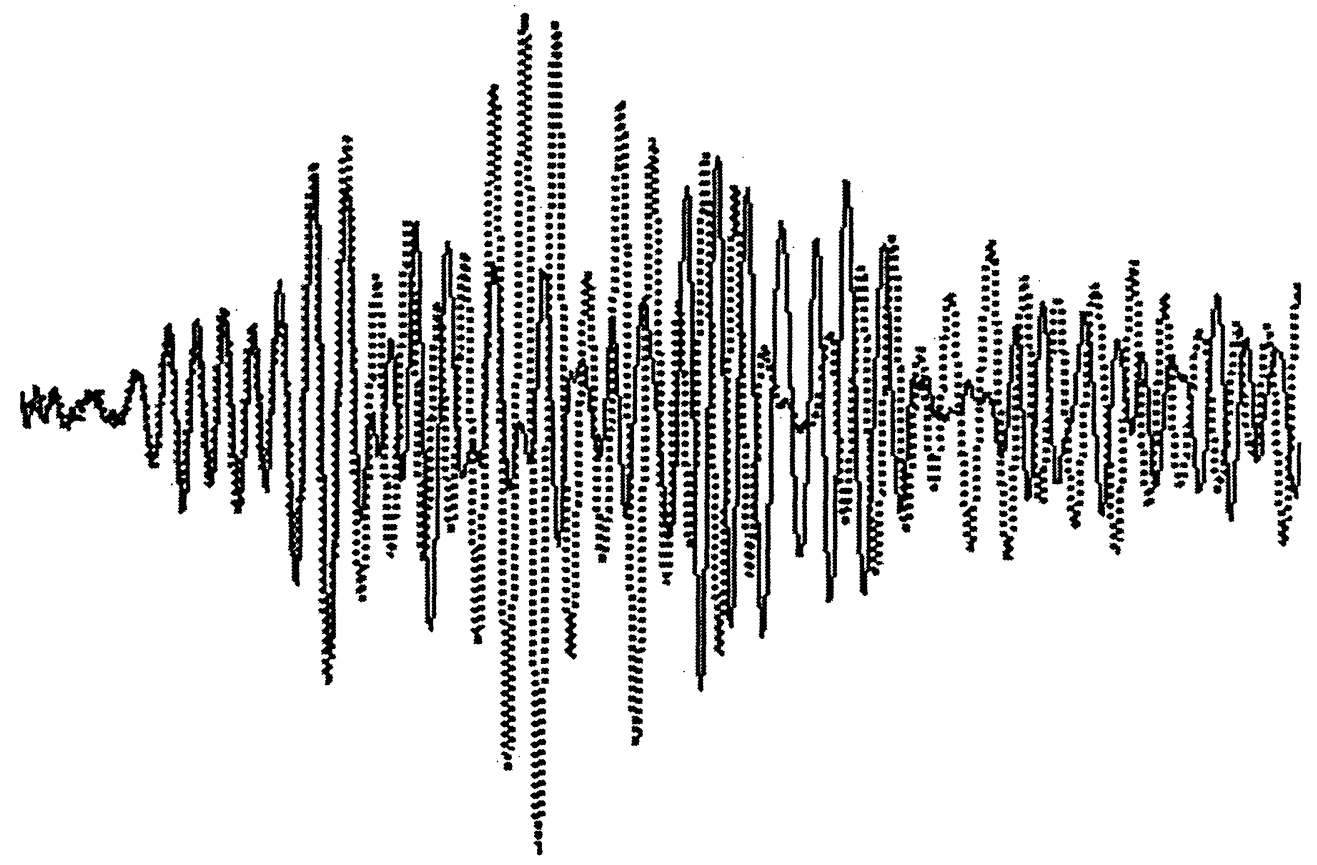

Figure 2.23 No Phase Shift after Platform is Stabilized

for Golay Pair [1,1], [-1,1] close to the backscatter signals from liver tissue

For the prior half bits in Golay code pairs we used (in Section 2.2.1.2) are the exact same. The beginning parts of the backscatter signals for Golay code pairs are the same also. This good property can be used to compensate the phase shift between the Golay code pairs. This compensation method is suitable to the reflection signals (Figure 2.22) and the signals from the real tissue(Figure 2.23). But it does not work for the signals from phantom that are too long and complex to compare. 


\section{Chapter 3 Results}

\subsection{Introduction}

There are two parts in this chapter. In the first part I present simulation results for binary coded excitation methods. In the second part I will present some results from testing phantoms (materials used to emulate tissue backscatter) and real tissue using our binary coded high frequency ultrasound system. All my simulations and analysis of experimental results are implemented in MATLAB.

\subsection{Theoretical Simulation Results for the Binary Coded Excitation}

The models used in the simulations such as sparse spacing, dense spacing, with attenuation, with plain :attenuation, with frequency dependent attenuation and different modulated signals are discussed in Chapter 2. We just present some important results. In all simulations the $\mathrm{x}$-axis is the depth in millimeters $(\mathrm{mm})$ and $y$-axis represents the amplitude of signals. In all the simulation results, the amplitudes of signals have no physical unit and are relative values. We normalize the maximum amplitudes of input signal to unit 1 to compare our results.

\subsubsection{Sparse spacing models}

In the sparse spacing modle, the distances between two adjacent scatterers are long enough to identify each backscatter event. The sparse spacing makes it easy to analyze if the coded signal implementation is correct or not and help us understand how the different models work. In simulations we choose the code length of 8 as default length. 


\subsubsection{Pulse Compression without Attenuation}

Both reference code and Golay coded signal are shown in Figure 3.1. The method to generate both signals is described as follows. First we choose the Golay code we want to use in simulation as discussed as Section 2.2.1.2. For example, $\{-1,-1,-1,1$, $1,1,-1,1\}$ is our choice. A sine signal is selected as the modulated signal and the bipolar method is used (Section 2.2.1.3). When the bit of code is -1 , one cycle of inverse sine is generated and when the code is 1 , one cycle of sine is generated; the Golay coded signal is shown in Figure 3.1(b). The reference code as shown in Figure 3.1(a) is the code itself but the number of sampling points for each bit of code is as same as the number of one cycles of the sine signal.

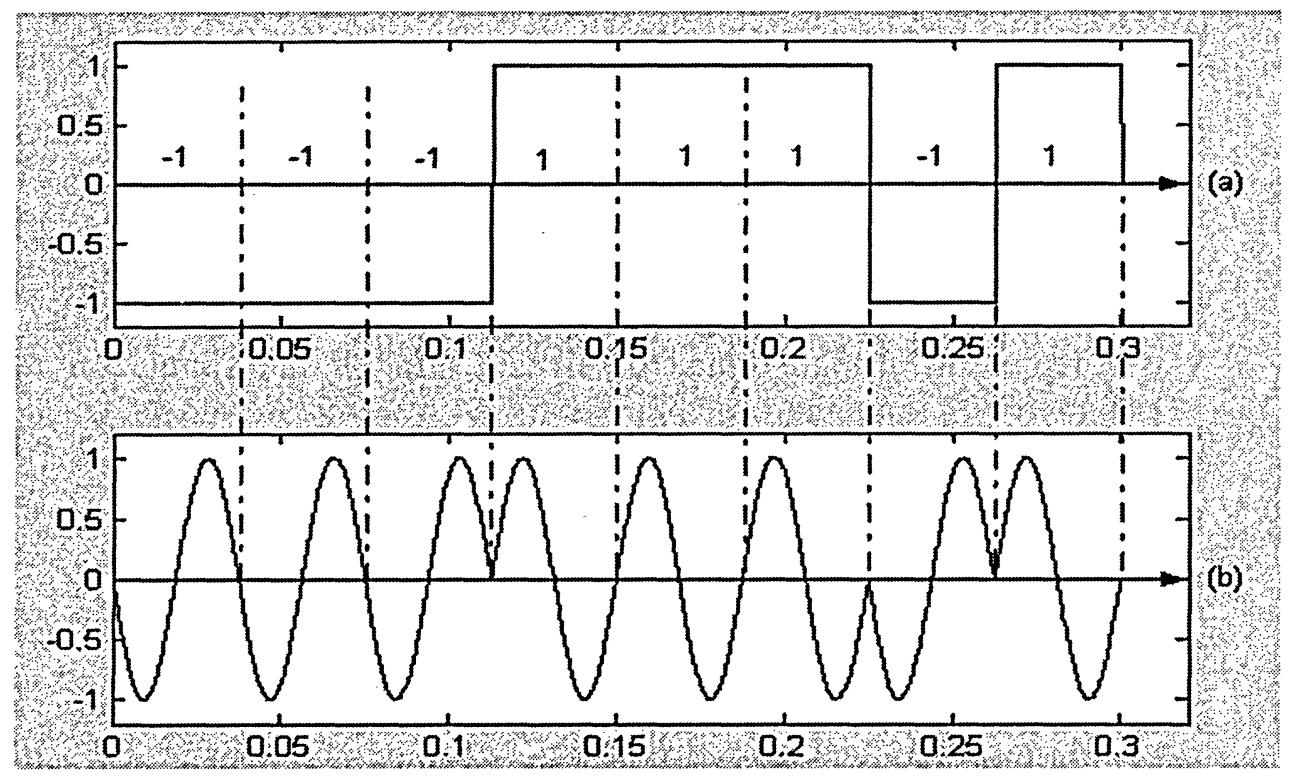

Figure 3.1 (a) One Cycle Code Reference Processing Reference Signal

(b) Golay Coded Signal. Code length 8 and code is [-1-1-1 $1111-11]$

Figure 3.1 (a) illustrates one cycle code processing reference. Compared with the examples in Section 2.3.4, this reference signal has zero separation between codes for there is no separation between neighbor pulses. 
A one-dimensional scattering model is used to simulate backscatter signals (details in Sections 1.7.5 and 2.3.1). We set the scatterer spacing to $0.35 \mathrm{~mm}$, as in Figure 3.1(b). The backscatter signal is shown in Figure 3.2. We notice the amplitude for all the backscatter signals is 1, which is the same value as the input signal in Figure 3.1 (b). When zooming in any portion of the backscatter signals, the decoded result is shown in Figure 3.2(b), which is the same signal as the original input signal as Figure 3.1 (b). The result is what we expect by one-dimensional scattering model.

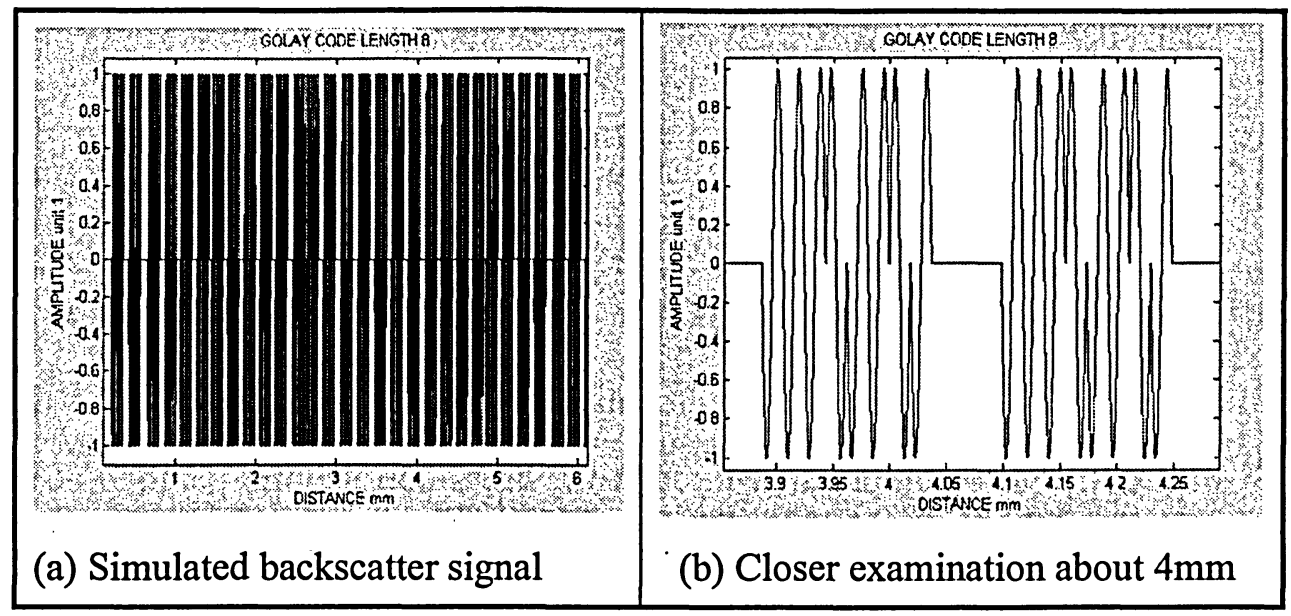

Figure 3.2 Backscatter Signal from Sparse Spacing without Attenuation

In simulations, I just use code reference processing method (Section 2.3.4). In this method the extended codes (as Figure 3.1 (a)) are used as cross correlation reference to decode the backscatter signals (as Figure 3.2 (b)). The encoding results for Golay code are gotten. The same thing done for the complimentary Golay code (the complimentary Golay backscatter signal is cross correlated with its corresponding reference code) and the decoded result for complimentary Golay code is gained. When these two decoded signals are added together, the encoding result is formed (Figure 3.3).

Throughout my thesis, the encoding results are always described by the sum of Golay code backscatter cross correlating to its reference and its complimentary Golay code backscatter cross correlating to its reference. 


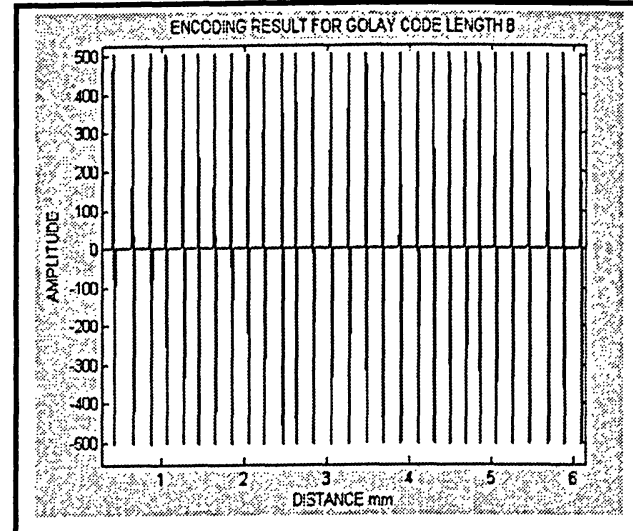

(a) Simulated backscattered signal after decoding (encoding result)

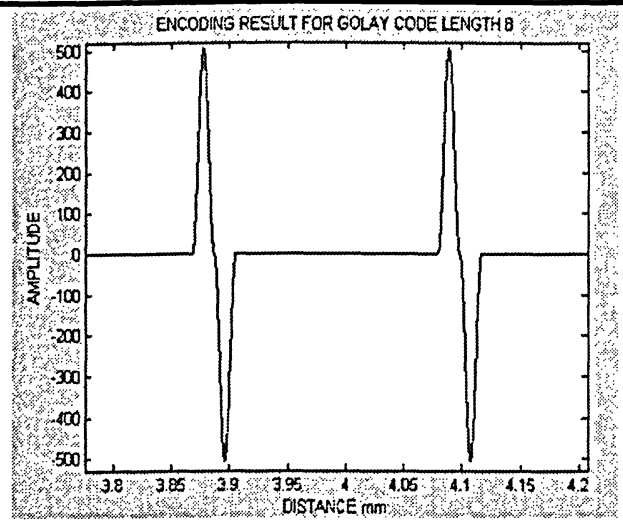

(b) Closer to examination of encoding result $(4 \mathrm{~mm})$

\section{Figure 3.3 Encoding results from Sparse Spacing without Attenuation}

(Code Length 8)

In Figure 3.3 we notice that the amplitude of the encoding results is a little more than 500. Pulse compression through the cross correlation operation accumulates the signal energy. The amplitude of reference signal is always 1 or -1 , so the cross correlation is similar to calculating the sum of sine signals. In our simulation the sampling number for one sine cycle is 100 . When we add half cycle of sine signal,

the first 50 positive values, together, that is, $\sum_{n=1}^{50} \sin \left(\frac{2 \pi}{100} n\right)$; the sum is 31.825 . The code length is 8 , so Golay and complementary Golay codes contain 16 cycles of sine signals. The sum multiplies the number of sine cycles, $31.825 \times 16$; the result is 509.2. This value is the same as the amplitude in our result. The encoding result looks like a sine which is the same as the encoding bit.

\subsubsection{Pulse Compression with Plain Attenuation}

As discussed in Section 2.3.2.1, we multiply the signal with an attenuation factor in the case of plain attenuation. For different scatterer points, we calculate their corresponding depth and adjust the signal amplitude with the attenuation factor. The 
backscatter signal using sparse spacing and plain attenuation is shown in Figure 3.4.

The encoding results are shown in Figure 3.5.

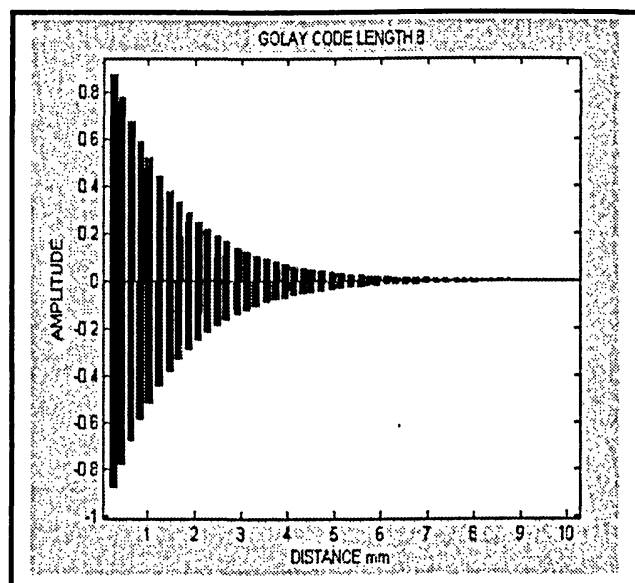

(a) Simulated backscatter signal

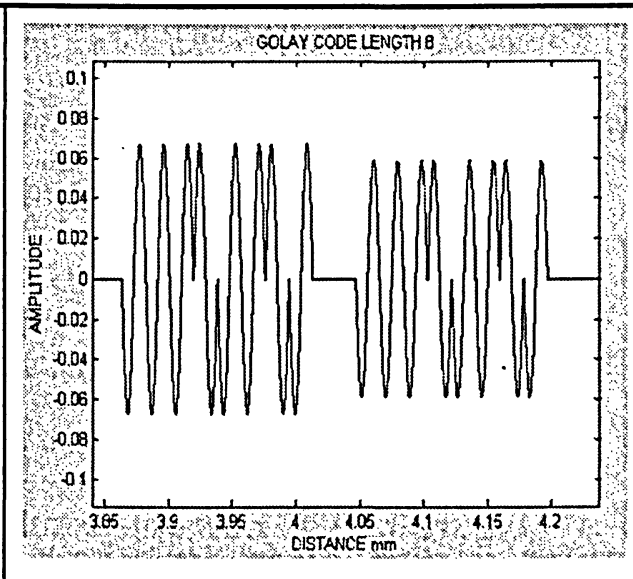

(b) Closer examination about $4 \mathrm{~mm}$

Figure 3.4 Backscatter Signal from Sparse Spacing with Plain Attenuation

When we compare the backscatter signals with and without attenuation, the only difference is that the signal amplitude decreases with depth. Plain attenuation has no effect on the shape of signal (Figure 3.5). The result from plain attenuation is similar to that from without attenuation; the difference lies in the decreased signal strength with depth. 


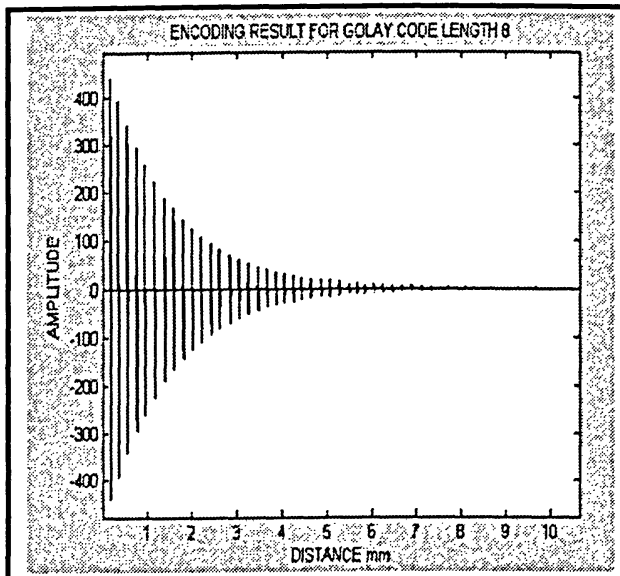

(a) Simulated backscattered signal after decoding (encoding result)

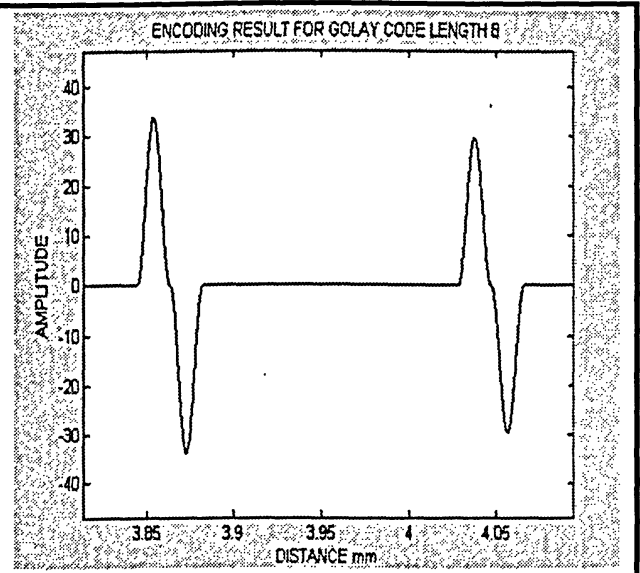

(b) Closer to examination of encoding result $(4 \mathrm{~mm})$

Figure 3.5 The Encoding results for Sparse Spacing with Plain Attenuation (Code Length 8)

When comparing the amplitude gains for different depths, the gains for different depths are almost same (Table 3.1), the small differences are due to the precision of data. The first row represents the scatterer depth; the second row lists the maximum value from the encoded backscatter signal. If one accounts for the attenuation factor $\mathrm{e}^{-\mu \mathrm{z}}$, the values are the same as in figure 3.4. The third row lists the maximum after pulse compression, this value just read from Figure 3.5; the last row is the ratio of output maximal to original maximal, which is called encoding gain. It is used to evaluate the effect of pulse compression.

Table 3.1 Encoding Gains for Different Depths with Plain Attenuation

\begin{tabular}{|l|l|l|}
\hline Location depth (in mm) & $0.2 \mathrm{~mm}$ & $3.4 \mathrm{~mm}$ \\
\hline Input max. & 0.765 & 0.0107 \\
\hline Output max. & 386 & 5.5 \\
\hline Encoding Gain & 505 & 510 \\
\hline
\end{tabular}




\subsubsection{Frequency dependent attenuation}

The methodology used to simulate frequency dependent (FD) attenuation is discussed in Section 2.3.2.2. The backscatter signal of code length 8 with FD attenuation is shown in Figure 3.6. The entire signal is shown in part (a) and close up regions are shown in part (b) and part (c).
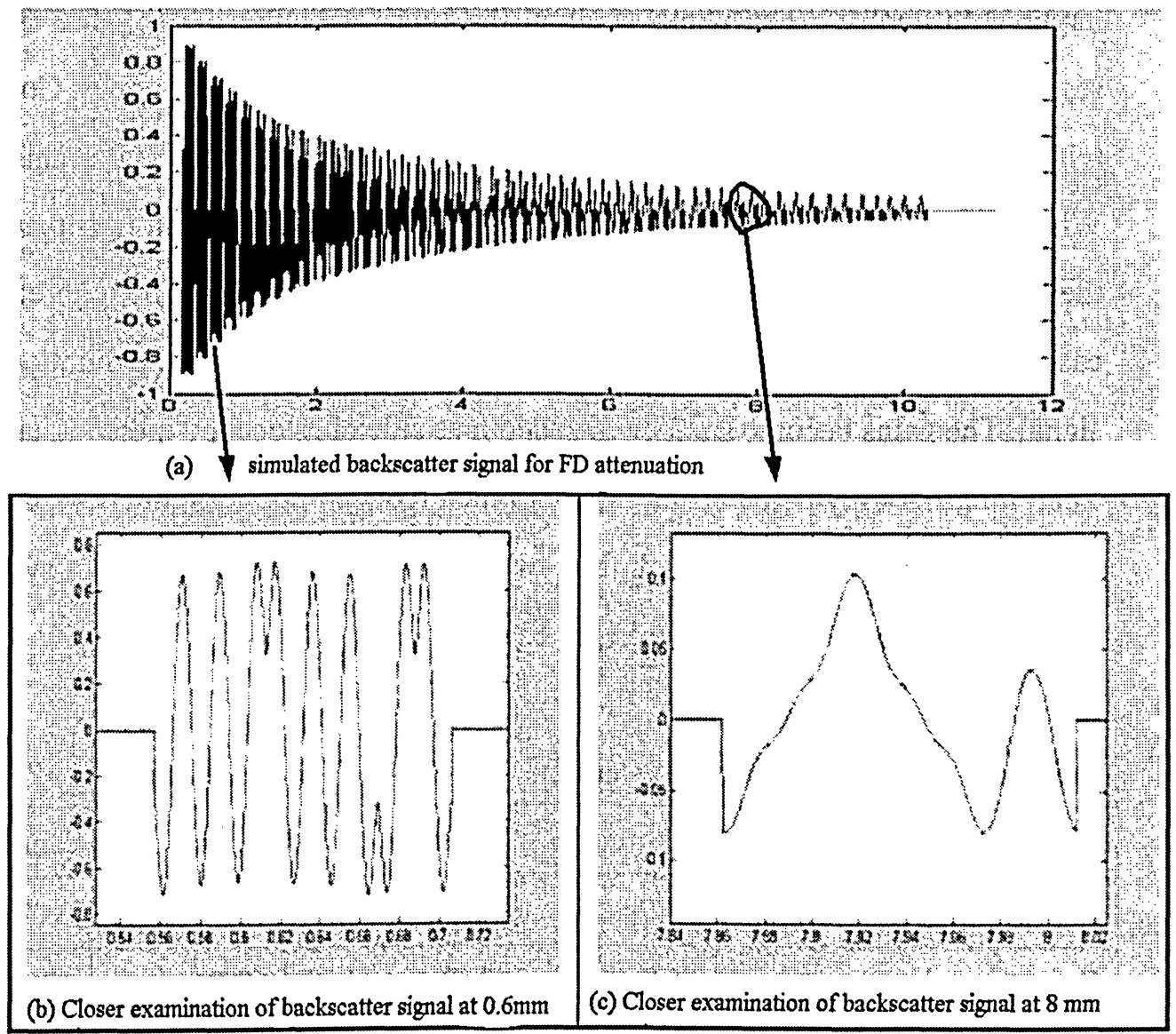

Figure 3.6

Backscatter Signal from Sparse Spacing with Frequency

Dependent Attenuation

In Figure 3.6 (b) and (c) the distances along $\mathrm{x}$ axis are almost the same so they show us an entire cycle of coded Golay signal. The distortions with depth are easily identified.

When the depth is about $0.6 \mathrm{~mm}$ (Figure 3.6(b)), at the locations of phase inversion 
the valleys are reduced due to the loss of the high frequency signal components. When depth is about $8 \mathrm{~mm}$ (Figure 3.6(c)), the signal is distorted beyond recognition due to loss in high frequency components of signal.

The encoding results using FD attenuation are shown in Figure 3.7. There are sidelobes between every scattering event and the amplitude of the sidelobes become larger with depth. When depth is about $0.6 \mathrm{~mm}$, as Figure 3.7 (b), the encoding result is similar to that of plain attenuation, but there are small sidelobes. Compared with the amplitude of signal about 400 , the amplitude of sidelobe is 9 at this depth. When depth is about $8 \mathrm{~mm}$, as in Figure 3.7 (c), the encoding result is not similar to the plain attenuation any more. Sidelobes (amplitude 11.2) cannot be ignored in this case. The time duration of sidelobes is even a little longer than the duration of the signal. The gains become smaller with depth as sidelobes occupy more signal energ. Therefore, not only is the encoding gain much reduced due to frequency dependent attenuation, but also the resolution of the binary coded excitation system is degraded by the sidelobes between the backscatter.

Table 3.2 The Gain for Different Depths with FD Attenuation

\begin{tabular}{|l|l|l|l|l|}
\hline Location depth (in mm) & $0.2 \mathrm{~mm}$ & $3 \mathrm{~mm}$ & $6 \mathrm{~mm}$ & $9 \mathrm{~mm}$ \\
\hline Input maximum & 0.894 & 0.305 & 0.155 & 0.0859 \\
\hline Output maximum & 464 & 163.5 & 68.8 & 33.4 \\
\hline Encoding Gain & 520 & 536 & 443 & 388 \\
\hline
\end{tabular}

In Table 3.2 sometimes the maximal gain is more than 536, even greater than without attenuation. The reason is that the distorted signal energy in the FD dependent attenuation model happens to be in the same phase with the signal as the instantaneous signal amplitude value is used. The trend of encoding gain is to decrease with depth. While the signal amplitude is not a good method to evaluate encoding gain, it is helpful for us to analyze our sparse spacing model and the 
quartz reflection in our experiment.

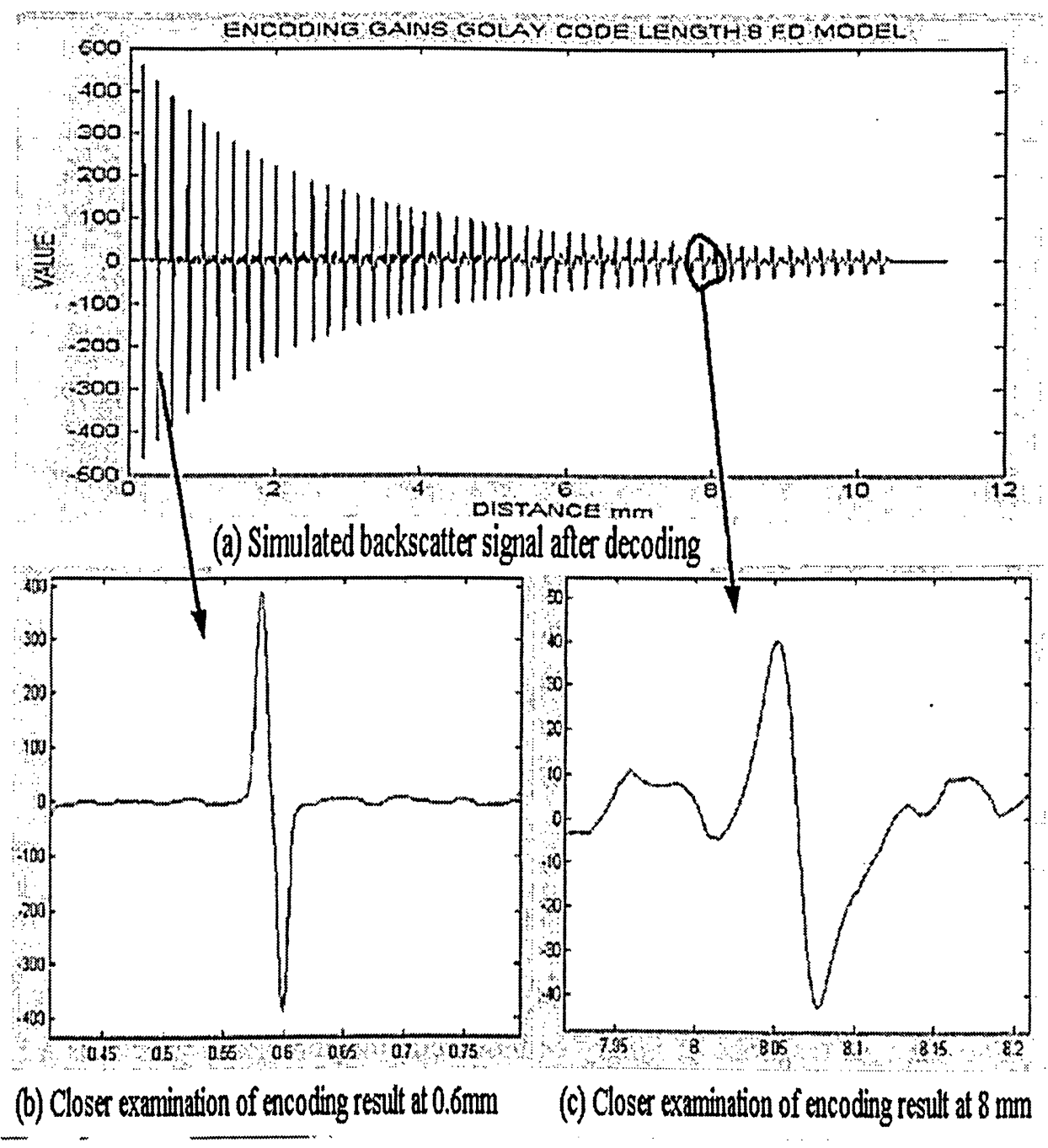

Figure 3.7 Encoding resultss for Sparse Spacing with FD Attenuation (Code Length 8)

\subsubsection{Effect of code length using sparse spacing models}

Similar simulations for code length 2, 4 and 16 were also performed. Without attenuation and with plain attenuation, the results from simulation are almost the same. When two sequences of Golay code and complimentary Goaly code are used, the encoding results always looks like a sine signal. The only difference is the amplitude of the encoding results. The amplitude gains for different code lengths are found to be linearly proportional to the length of the code, as we expected. 


\subsubsection{Impulse Response for the Coded Excitation Method}

The definition of impulse response is the mathematical function that describes the output waveform that results when the input is excited by a unit impulse. In the simulations we send pairs of unit signal (Golay code sequence and complimentary Golay sequence). After the binary decoding method (the sum of Golay code backscatter cross correlating to its reference and its complimentary Golay code backscatter cross correlating to its reference), the encoding results are obtained. Therefore, the encoding results are regarded as the impulse response for the coded excitation method in different attenuation models.

In Figure 3.3(b) and 3.5(b), the encoding result is similar to a sine, the chosen unit in the Golay sequence (Figure 3.1). After pulse compression, a perfect sine pulse replaces a sequence of sine signals in our result. The impulse response of the system approaches a $\delta$ function. For the plain attenuation or without attenuation models, the binary coded excitation method is very suitable as a method to increase the signal. The impulse response of binary coded excitation for these systems is a $\delta$ function. For biological tissues, significant FD attenuation exists. As shown in Figure 3.7, the

result is not a perfect sine any more. Significant sidelobes exist, reducing the encoding gain and degrading the system axial resolution. These sidelobes increase with depth.

\subsubsection{Dense Spacing Models}

\subsubsection{Without Attenuation}

When we change distance between scatterers from $0.35 \mathrm{~mm}$ to $7 \mu \mathrm{m}$, the result for the same Golay coded signal is shown in Figure 3.8 (Golay code $\{-1,-1,-1,1$, $1,1,-1,1\})$. The amplitude of the input coded signal is in range -1 to 1 in Figure 
3.1; the amplitude of backscatter signal is in the range of -2 to 2 . With no attenuation, the statistical amplitude of signal is almost the same with depth.

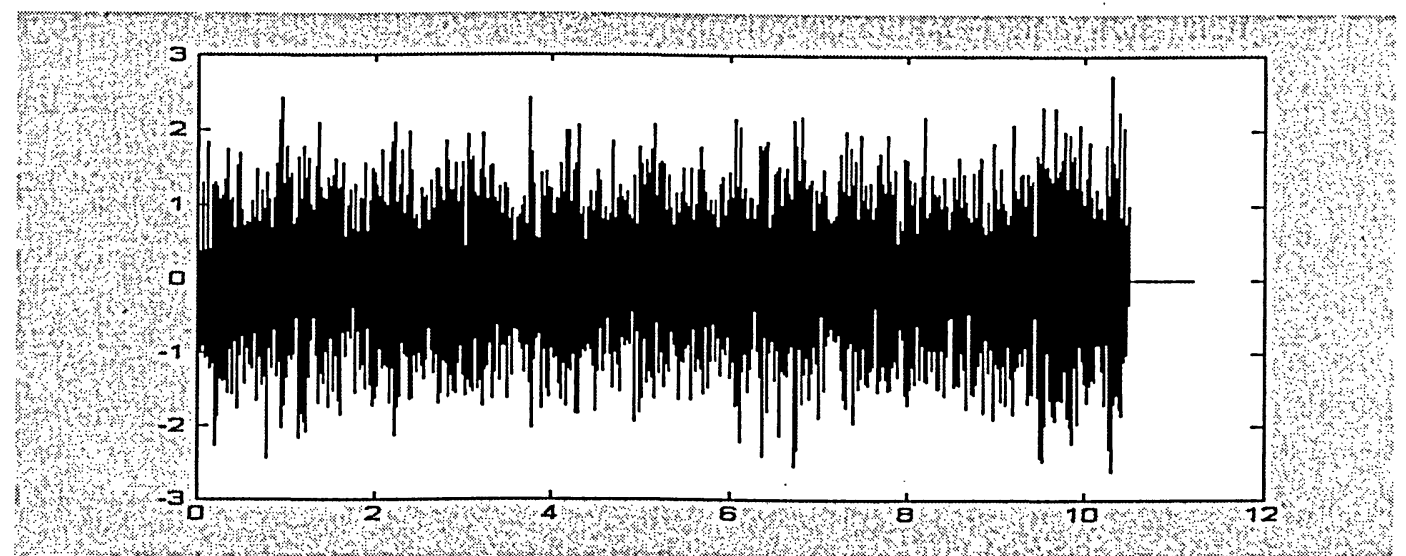

Figure 3.8 Backscatter Signal for Dense Spacing without Attenuation (Code

\section{Length 8)}

The encoding results of the backscatter signal for dense spacing without attenuation model are shown in Figure 3.9. The values in the beginning and end are artifacts (above 500). In the middle of the signal the peak value is 320 .

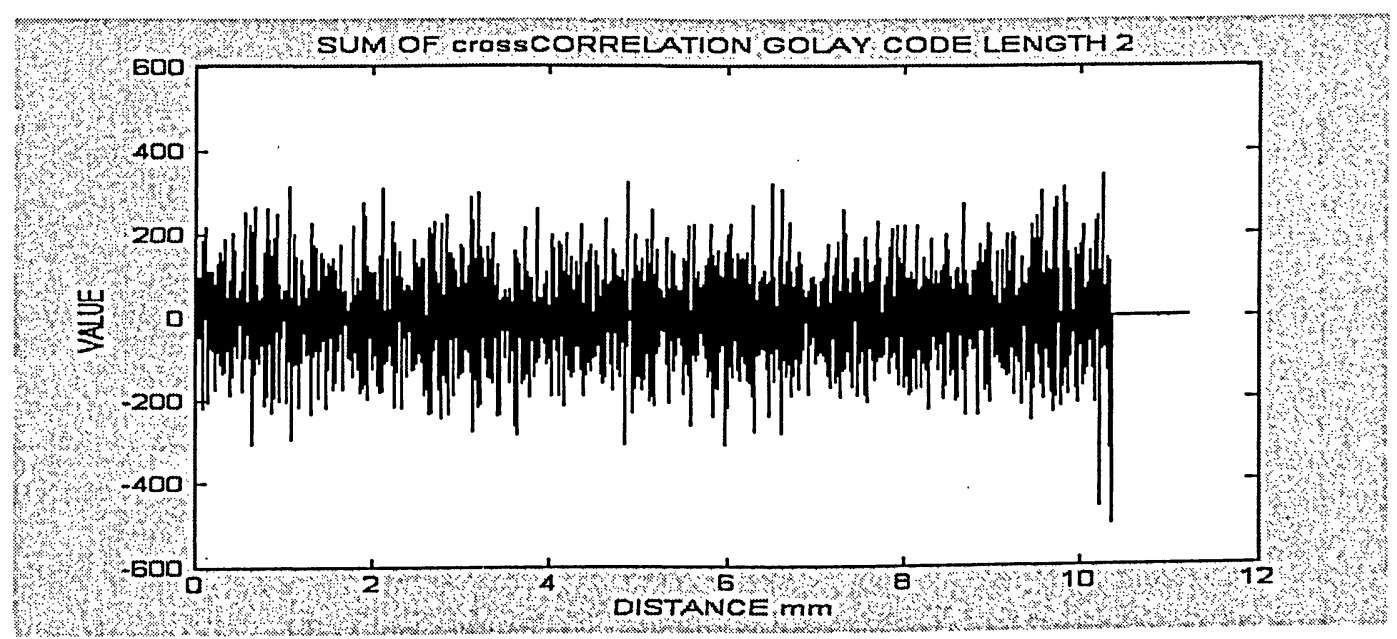

Figure 3.9 Encoding resultss of Dense Spacing without Attenuation (Code Length 8)

Standard deviations (STD) of the signals can be thought to represent the signal energy. In Figure 3.10 (a), the STDs of original backscatter signals for four different 
code lengths are plotted: thin solid line is for code length 16; thick dot line is for code length 8 (signal in Figure 3.10); thick solid line is for code length 4; and thin dot line is for code length 2. As later we will study the similar properties for plain attenuation and FD attenuation models, we divide the signal into 32 segments, based on the length of the code. For code length 8 , its duration is $0.2 \mu$ s and length is $0.3 \mathrm{~mm}$, so the signal of $1 \mathrm{~cm}$ depth is divided into 32 segments, and each segment have the same length as the length of encoded signal: $0.3 \mathrm{~mm}$. In order to compare the results for different code lengths, the signal is always divided into 32 segments. In the model without attenuation we do the same thing by dividing whole signal into 32 segments. The $\mathrm{x}$-axis represents the different segments according to depth, and $y$-axis denotes the STD values of the backscatter.In Figure 3.10 (b), we list the STD of encoding results for four different code lengths.

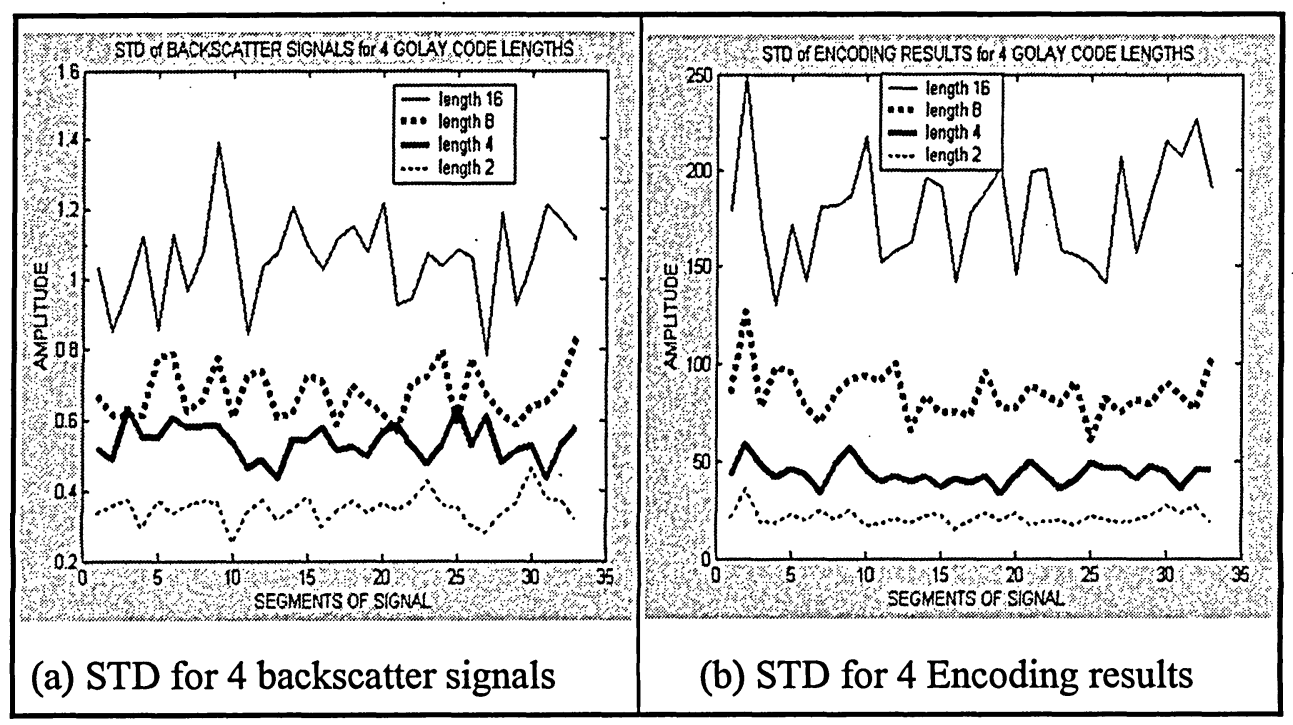

Figure 3.10 The STD for Four Code Length. All Data Come from Dense Spacing without Attenuation.

The average STDs of original backscatter for codes length $16,8,4,2$ are listed in Table 3.3. The Gains for code lengths 2 and 4 are linearly increase. 
Table 3.3 The Relation of Code Length and Encoding Gain. All Data from dense Spacing without Attenuation at $6 \mathrm{~mm}$ Depth

\begin{tabular}{|l|l|l|l|l|}
\hline Code length & 16 & 8 & 4 & 2 \\
\hline Input STD & 1.03 & 0.67 & 0.52 & 0.34 \\
\hline Output STD & 178.80 & 87.67 & 47.70 & 20.93 \\
\hline Encoding Gain & 172 & 131 & 92 & 61 \\
\hline
\end{tabular}

\subsubsection{Plain attenuation}

The same simulation is implemented as described in Section 3.2.1.2 after the distance between the scatterers is changed from $0.35 \mathrm{~mm}$ to $7 \mu \mathrm{m}$. The result of backscatter signal is shown in Figure 3.11 (a). After the same reference code is used in the cross correlation operation, the encoding results are shown as Figure $3.11(b)$.

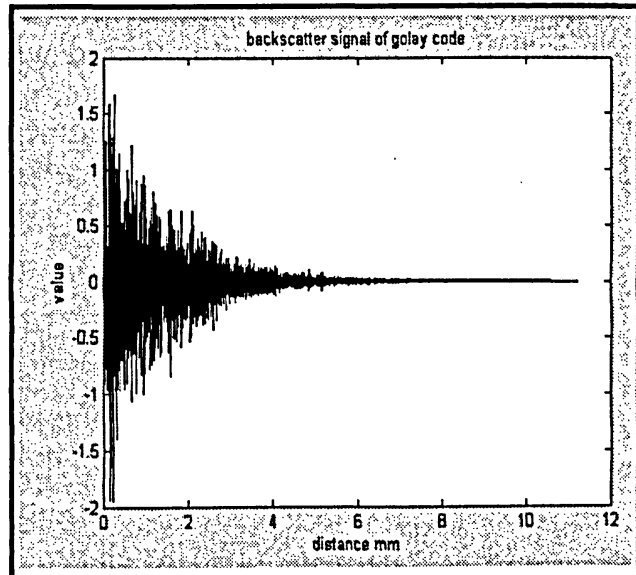

(a) Backscatter Signal from Golay sequence

Figure 3.11

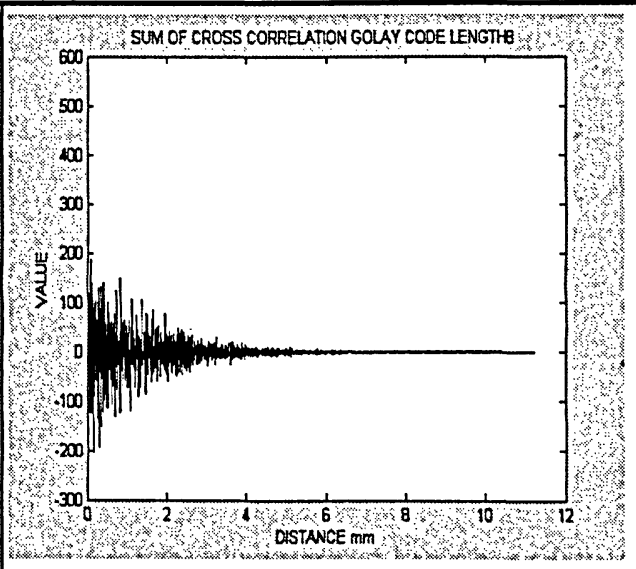

(b) backscatter signal after decoding

\section{Backscatter Signal in Dense Spacing Model with Plain Attenuation (Code Length 8)}

In the beginning of the encoding results, there is an artificial large amplitude value. 
This is similar to the case without attenuation. After we separate the signal into 33 segments, the STD for both signals are shown in same type lines in Figure 3.12. There is a large increase in the STD indicating a substantial encoding gain.

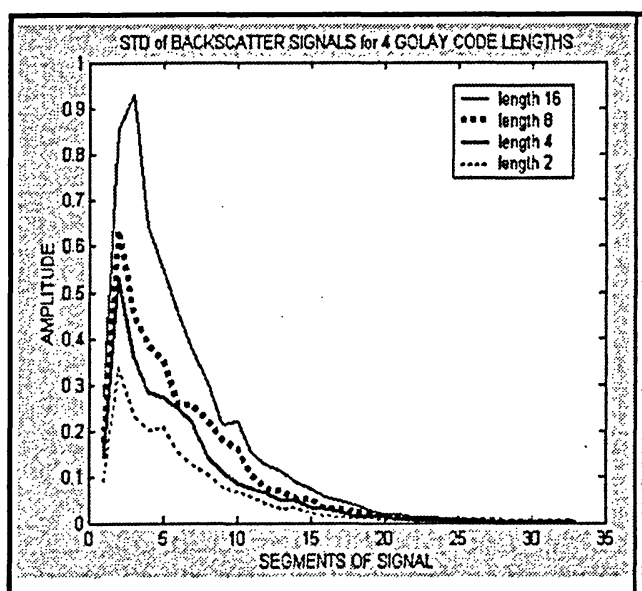

(a) STD for backscatter signals

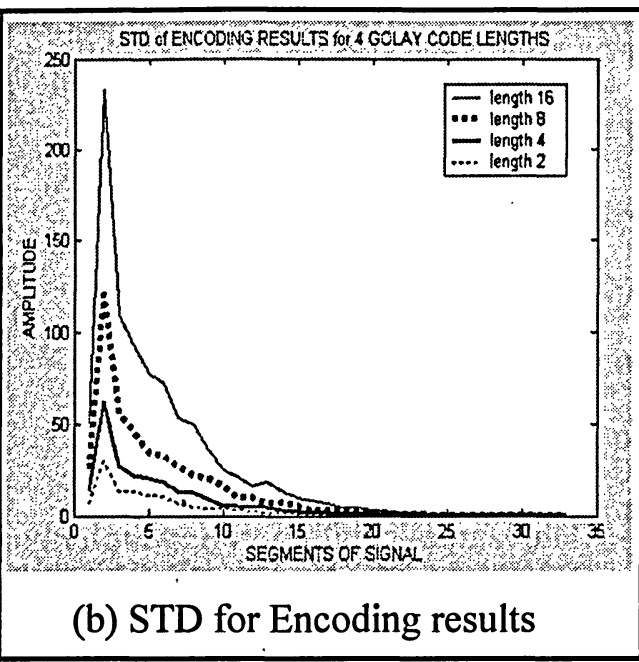

(b) STD for Encoding results

Figure 3.12 STD for Four Code Length Dense Spacing with Plain Attenuation.

The results for the model with plain attenuation are almost the same as those from without attenuation; the only difference is that attenuation factor varied with depth exists in plain attenuation. This result is the same obtained for sparse spacing models.

\subsubsection{Frequency dependent (FD) attenuation}

With FD attenuation, the results are shown in Figure 3.13 (for the backscatter signals) and Figure 3.14 (for STD of these signals). 


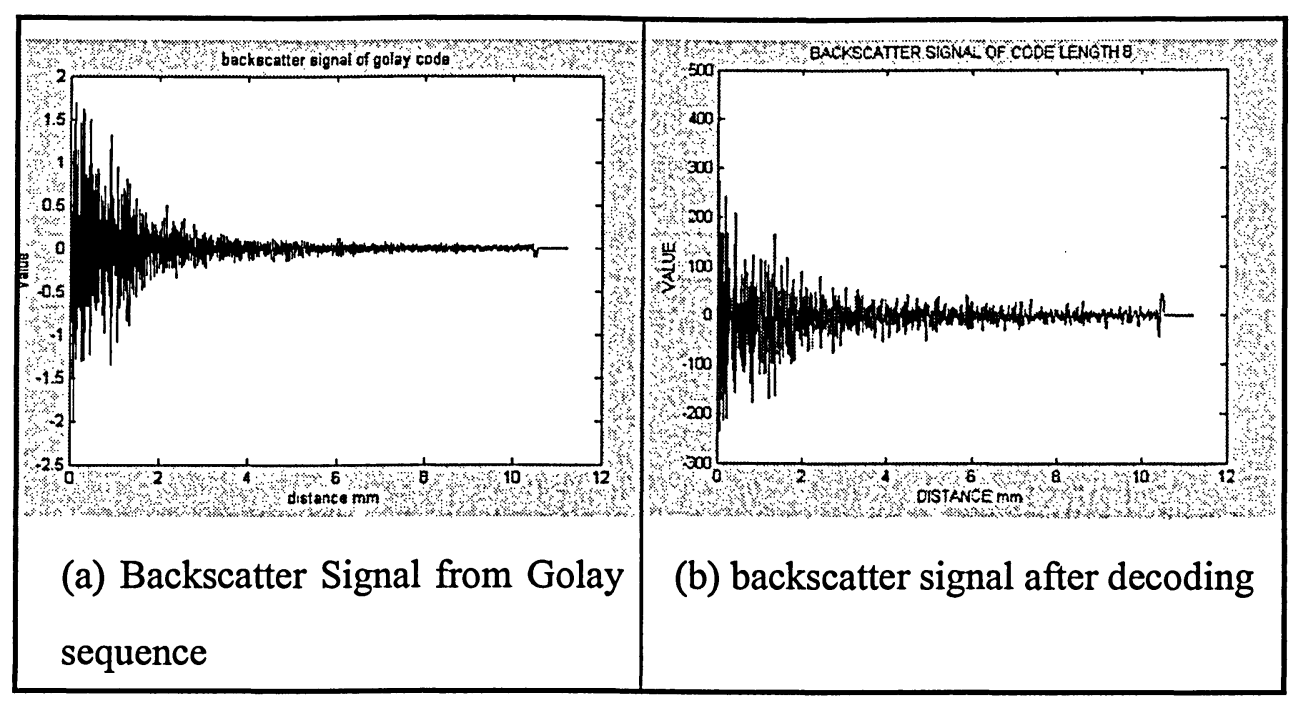

Figure 3.13 Backscatter Signal for Dense Spacing with FD Attenuation (Code Length 8)

When the backsactter signals for the plain attenuation model are compared with the FD attenuation model backscatter (Figures 3.11 and 3.13), the amplitudes of signals in depth for FD attenuation are greater than these for plain attenuation. In the plain attenuation simulation results the signals from 6 to $10 \mathrm{~mm}$ are too small to identify (as Figure 3.11(a)), but the signals can be identified in the same depth for FD attenuation (as Figure 3.13(a)). The amplitudes for both signals from 1 to $6 \mathrm{~mm}$ are almost the same. The reason is that in FD attenuation model the signals lose less energy in the low frequency portion of the signal. For details see Section 2.3.2.2 and Figure 2.8.

When studying the STD for the four different code lengths in Figure 3.14, we find the trends of STD for different code length in two figures differ. The STD for code length 2 (thin dot line) sometimes is greater than that of the other codes for deep locations. The lower frequency signal contents for code length 2 are greater than these for code length 4,8 and 16 so the code length 2 retains energy in depth better than the other three. But after the processing, as Figure 3.14 (b) illustrates, the STDs of encoding results for the four code lengths are in order of length (the longer the code is, the greater the STD). The longer code can accumulate more energy than 
the shorter one and the "accumulation effect" is greater than the loss in high frequency.

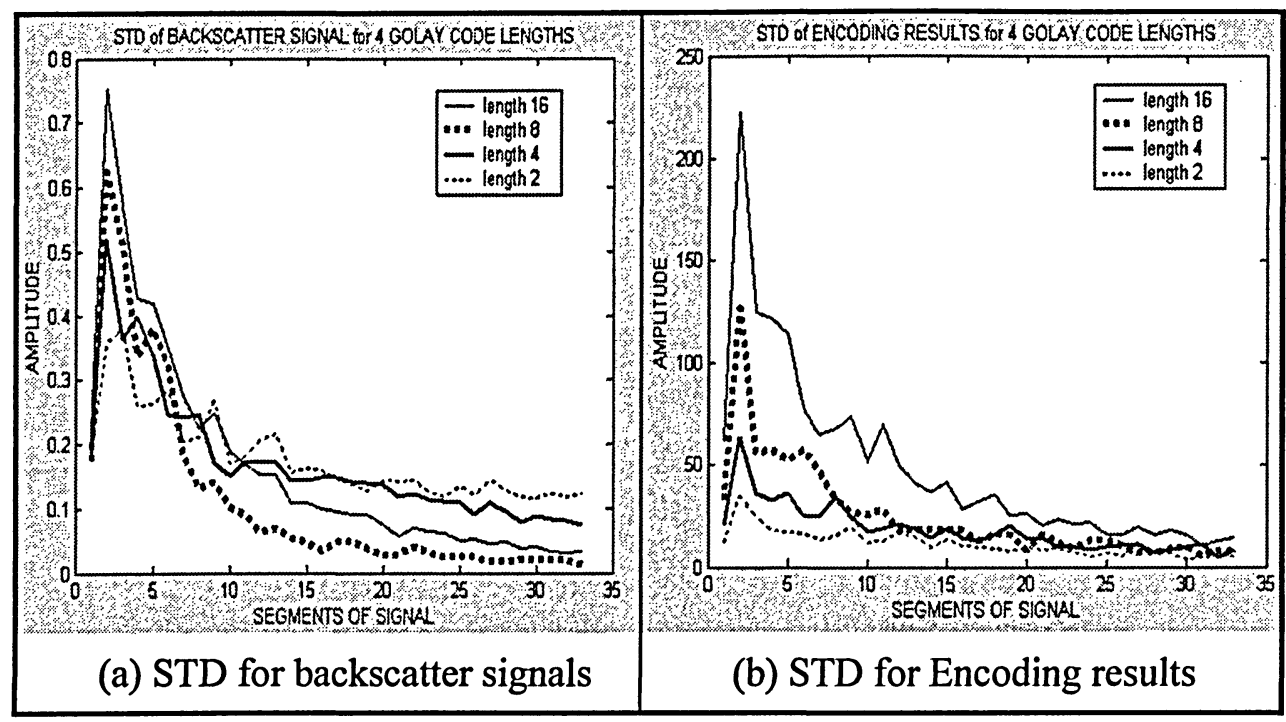

Figure 3.14 STD for Four Code Length (Dense Spacing with Plain

Attenuation)

\subsubsection{Simulations with closely spaced scatterers}

As for the sparse models, the signal amplitudes for four different code lengths are compared. The closely spaced scatterers emulate the tissue backscatter. By comparing the STD ratio of the four different code lengths, we represent the gain accomplished with the coded methods. In Figure 3.15 the STD ratios are shown for both the without attenuation and plain attenuation models. The trend for the STD of the backscatter signals and encoding results for both the plain attenuation and without attenuation simulations are almost the same and the ratio for each segment is almost constant (Figures 3.10 and 3.12). We find the ratio increases linearly with code length, and the ratios for both models are almost the same. 


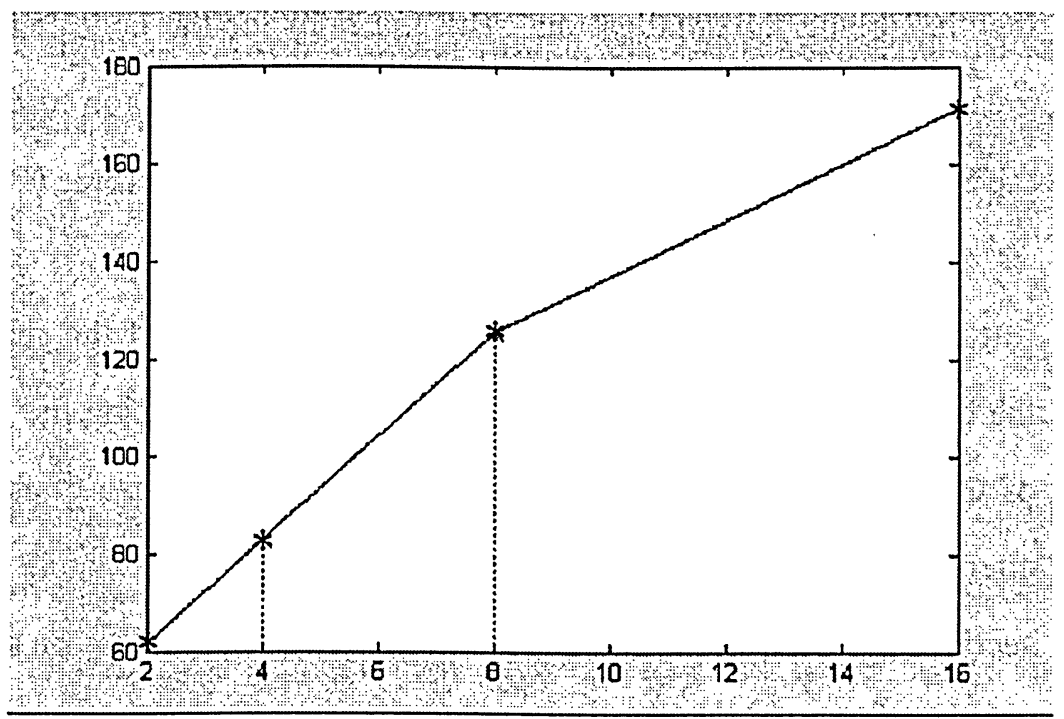

Figure 3.15 Average STD Ratio for Four Code Lengths

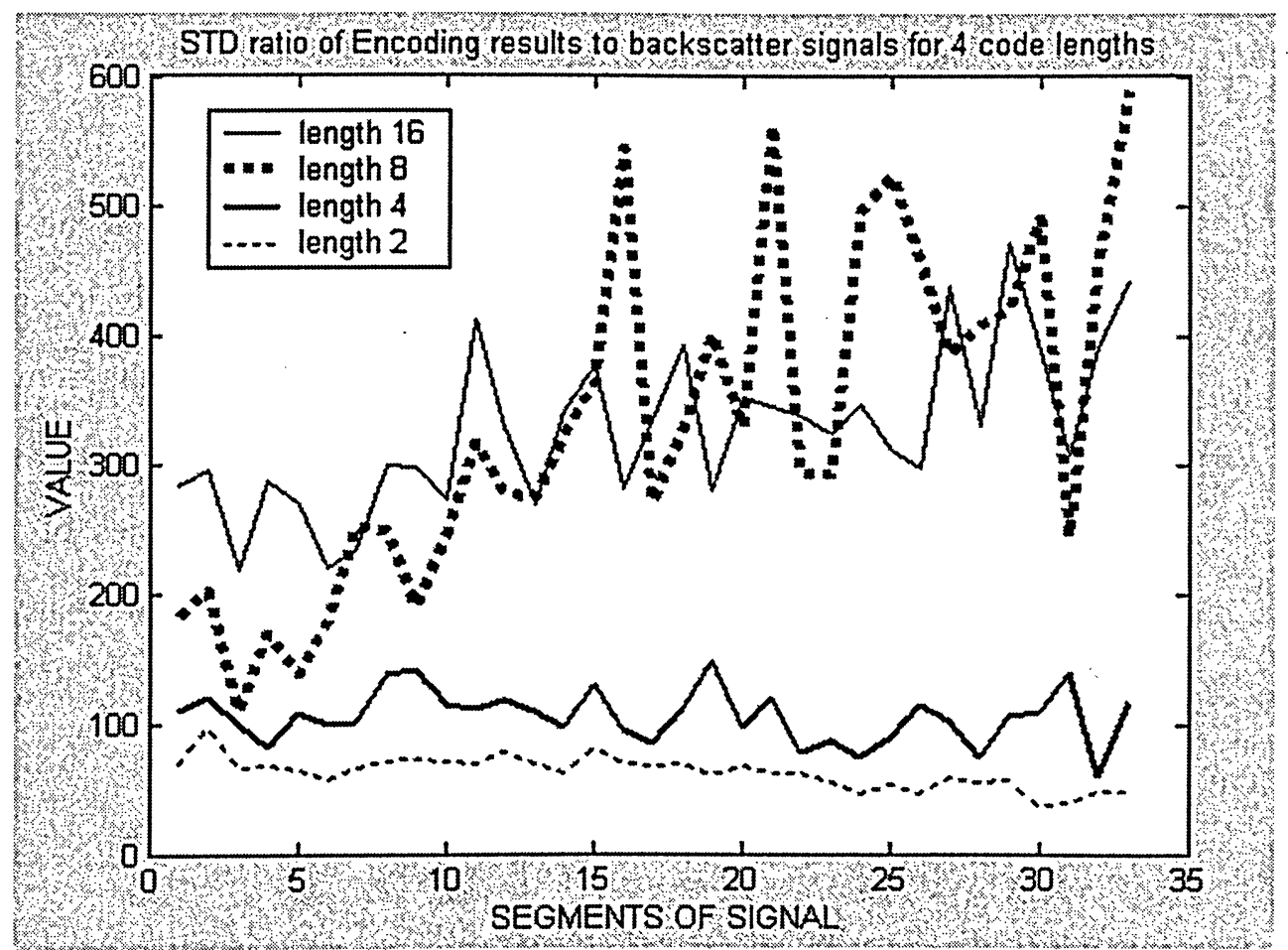

Figure 3.16 The Ratio of STD of Four Code Length (Dense Spacing with FD Attenuation )

For the FD attenuation model, the trend for four code lengths is different (Figure 3.16). The ratio for the different code lengths increases with code length, code 
length 2 being the smallest one. But the ratio for the code length 8 sometimes is greater than code length 16 . The reason for large STD ratio of code length 8 is that the STD value of backscatter is much less than the other three (Figure 3.14 (a)).

In this section, simulation results were discussed. All of the simulations show that the binary coded excitation method is capable of increasing the amplitude and STD of the high frequency ultrasound signal even in the case of frequency dependent attenuation. The encoding gains generally increase with code length. In the next section, I present some results from the binary coded excitation system.

\subsection{Experimental results for the binary coded excitation system}

From this section on experimental results are presented. The reflection signals from a quartz plate are acquired first. The quartz reflection backscatter signals are similar to the sparse spacing model in Section 3.2.1, as they are easily to analyze and helpful for us to try different processing methods. Then some results from phantoms are presented. Phantom is a good substitute for real tissue as it has stable properties. Tissue decays rapidly with time and will change the properties of ultrasound backscatter signal. Another advantage for phantoms is that they are easily to dimmobilize in water but the real tissue floats. Water is used for transducer impedance matching as discussed in Section 1.5.3.2. In the last section signals from liver tissue are presented.

In the simulations, length 8 was chosen as the default code length. In the experiments the binary coded excitation using a code length 8 was not optimal (details in Section 2.4.2.1.). Since the encoding results of short code lengths have smaller sidelobes than longer ones, code length 2 was chosen as the default code length. In all figures with experimental results, their $\mathrm{x}$ axis represents the sample 
number. In all figures with experimental results, the $\mathrm{x}$-axis represents the sample number. Since the sampling rate is $5 \mathrm{GHz}$, the time between two points represents $0.2 \mathrm{~ns}$.

\subsubsection{Three Processing Methods}

In Section 2.3.4, three different processing methods were defined. In the simulations we used the second method, the one cycle code reference processing method. One cycle of sine per code was used to obtain the coded signal and the processing reference was either 1 or -1 for each bit code. It is not clear, however, which method is best. Therefore, the three methods are compared.

The first method is the reflection reference processing method, in which the backscatter from a quartz plate is used as the cross correlation reference. The second method is the code reference processing method, in which the code is used as a cross correlation reference and the reference length for each bit of code is one cycle of the center frequency. The third method is the code with phase reference method, in which three cycles of the are used center frequency for each bit in the reference and the reference is 1 or -1 based on the phase of the signal.

In the first method, the signal and its reference happen to be the same so the auto correlation operation replaces the cross correlation. When the reflection is used as the reference, the reflection is truncated as shown in Figure 3.18 (a) (solid line). Noise and sidelobes are truncated from the reference which improves the quality of encoding results. 


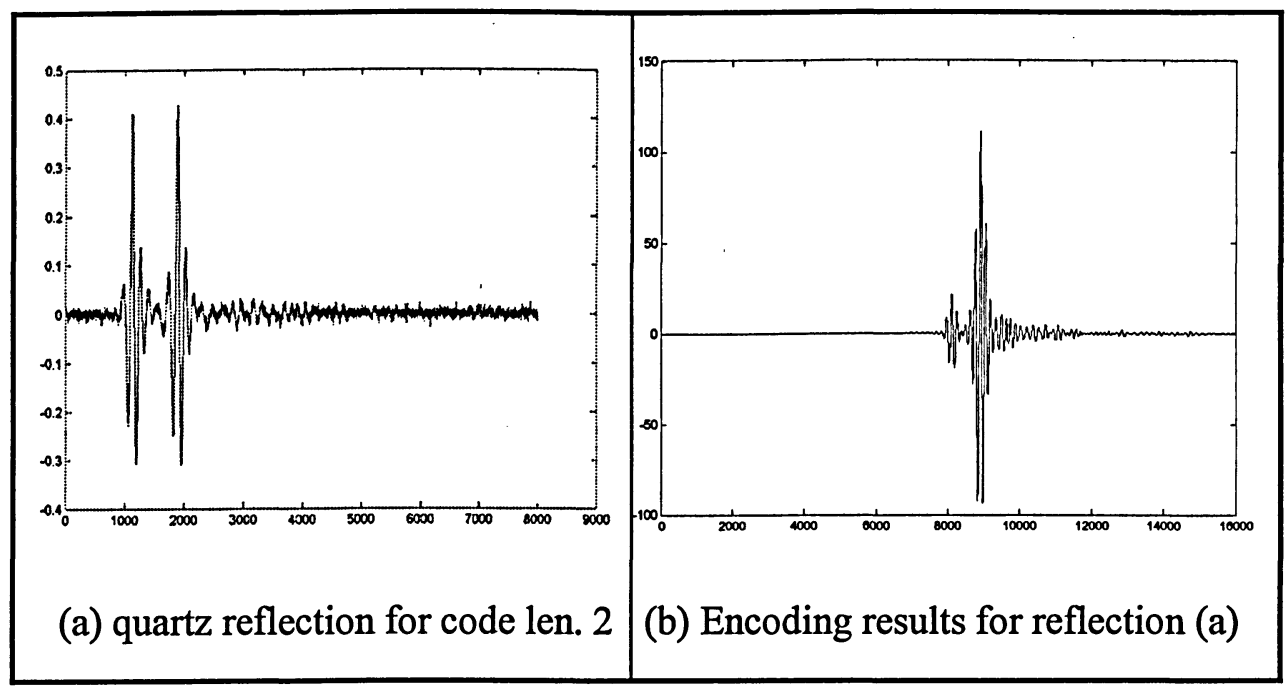

Figure 3.17 Signal and Encoding resultss using the Auto Correlation

\section{Method}

Figure 3.17 (a) shows the quartz reflection for code length 2 . The y axis is the signal amplitude in voltage (which is proportional to pressure), and $\mathrm{x}$ is the sample number. In Figure 3.17 (b), the encoding results for the reflection reference processing method with code length 2 are shown.

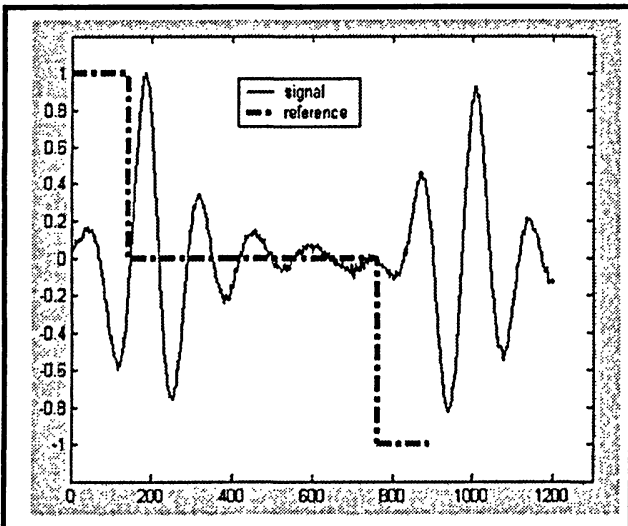

(a) Back signal and its reference

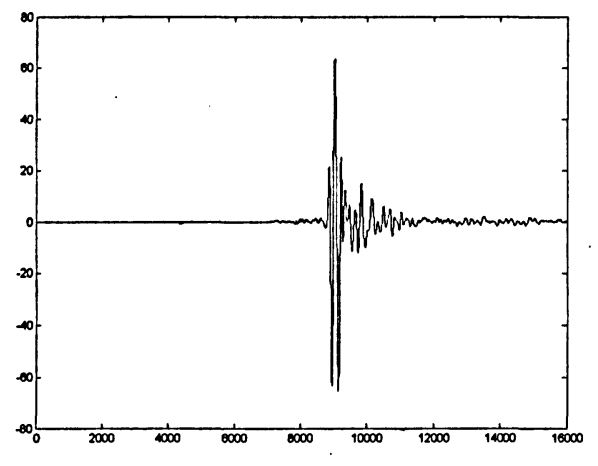

(b) Result in code reference method

Figure 3.18 Signal and Encoding results in Code Reference Method

Figure 3.18 (a) shows the quartz reflection for code length 2 and its reference for the one cycle code length. In Figure 3.18 (b), the encoding results for the one cycle code reference processing method code length 2 are shown. The processing 
reference is shown in Figure 3.18(a). The solid signal is signal from reflector; the thick dot dash line is the reference from code (details in Section 2.3.4). We call this method as code reference method.

The code with phase reference method is shown in Figure 3.19 (a), the solid signal is also the quartz reflection and its reference (the thick dot dash line) also comes from code but the phase of signal itself is considered (details in Section 2.3.4).

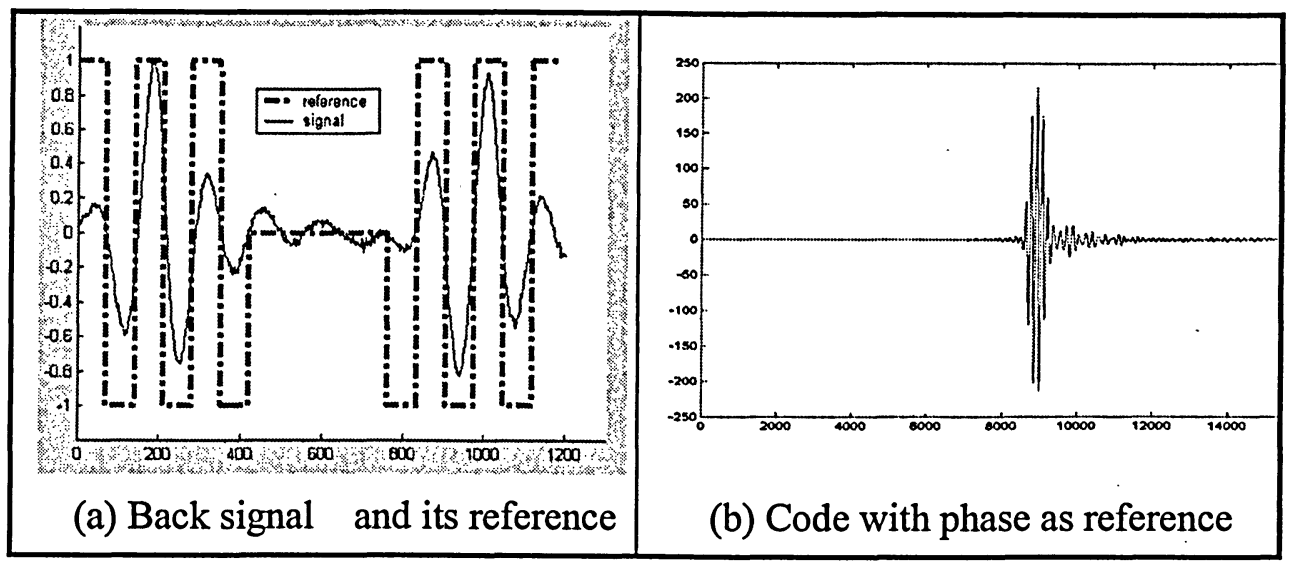

Figure 3.19 Signal and Encoding results in Code with phase Reference Method

When comparing the encoding results for these three methods (Figures 3.17(b), 3.18(b) and 3.19(b)), the maximal amplitudes of results are about 115 for the reflection reference, 63.3 for code reference and 214 for code with phase reference. The sidelobes for the code with phase method were the smallest. Therefore, we chose the code with phase reference method to analyze our results.

In Figures 3.17(b), 3.18(b) and 3.19(b), the amplitude of the beginning of these signals is zero. Before Matlab cross correlates two signals, it fills zeros into short signals to make them in the same length (zero padding). These zeros force the beginning of signals to be zero. In the following sections, we remove the padding from the encoding signals. The truncated signals are helpful for us to compare the different results. 


\subsubsection{Signal Encoding Gain}

In the reflection reference method, the maximal value for the auto-correlation is at the position where the signal and the reference overlap and the signal and reference are in the same phase, so all the products for positive and negative values are positive. The sum of the square of all the sample points for one pulse reflection is 28.8. For Golay and complimentary for code length 2 there are four pulses, the total is $4 \times 28.8=115.2$, which is the same as the maximal value in Figure 3.17(b).

The signal phase is considered in the code with phase reference method. When the processing reference and the signal at the position where they are the same phase as shown in Figure 3.20 (b), the maximum of the cross correlation operation is reached. For the reference and signal have the same phase, when the signal is positive the reference is positive; when the signal is negative, the reference is. The absolute amplitude of reference is 1 . The cross correlation is equal to the sum of the absolute value of all the amplitudes of all the signals. When we sum the absolute value of all the sample points for one pulse reflection, the result is 53.4. For Golay and complimentary Golay codes there are. four such cycles, so the total value is $53.5 \times 4=214$, which is the value of the maximum in Figure 3.19 (b).

In Section 3.2.1.1, the maximal amplitude is explained for our simulation results. During calculation we use the sum of just half cycle sine signal. The code reference method ignores the phase of sine signal; the maximal correlation value is at the position when half period phase shift exists between the sine signal and its reference in Figure 3.20 (a). If the reference moves to right continually, the half period of negative signal will add to the positive half period value and the reference is positive 1 so that the production of signal and reference is negative. The result become smaller with more negative signal added. When no phase shifts between the code reference and the signal, the sum is zero (the sum of half positive period with 
the negative half). The maximal value happens when code with phase reference and its signal have the same phase as shown in Figure 3.20(b). So code with phase reference method improves the amplitude as twice as the code with phase reference.

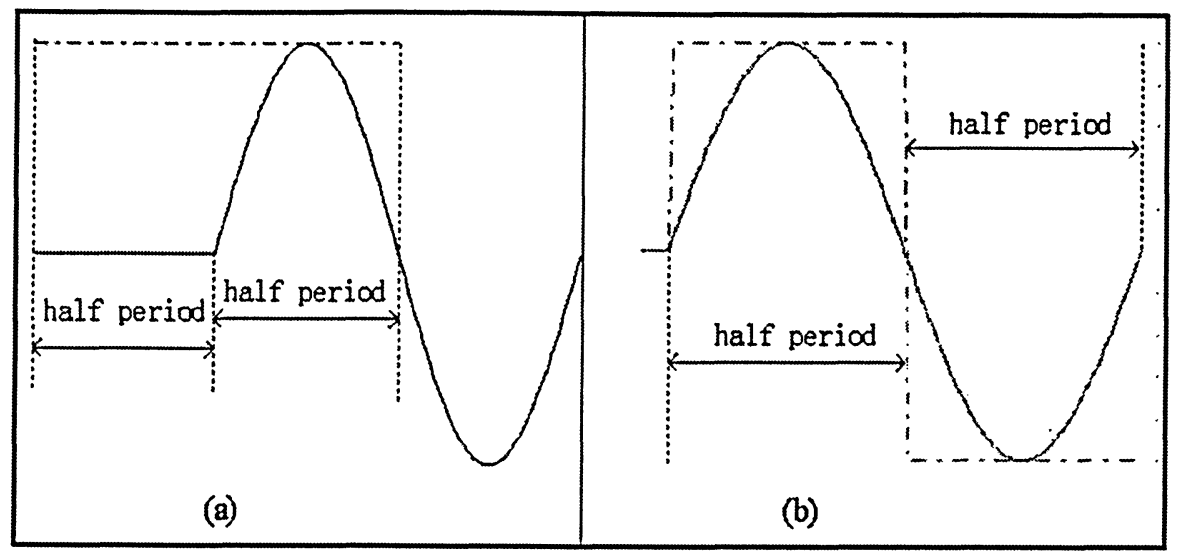

Figure 3.20 Maximal Correlation Positions for Code Reference and Code with Phase Reference

In Figure 3.20 the dash line is the reference code and solid line is the signal. Figure 3.20 (a) represents the code reference method which can reach its correlation maximum when half period phase shift exists. In Figure 3.20(b) the maximum occurs when there is no phase shift between the code with phase reference and signal. The code with phase method uses the whole signal, but the code reference method just uses half.

In Figure 3.17 (a), for the backscatter signal, we can use STD of three-cycle large peaks as our signal power and the STD before the first peak as our noise. The signal and noise power are individually 0.1383 and 0.0063 , so the signal to noise ratio is 22. When we do the same thing for the encoding results for three-cycle code with phase processing method (Figure 3.19 (b)), the signal and noise power are separately 116 and 0.36 , so the signal to noise is 320 . We choose the part of signal before the peak value as the noise for signal to noise ratio. This part has no sidelobes and is almost just white noise. So the gain of signal to noise ratio for 
binary coded excitation code length 2 is 15 times (12dB); for code length 4 the gain is $15 \mathrm{~dB}$. This value can be regarded as the maximum realizable improvement. These values happen to be the theoretical gain in the FM coded excitation method $^{[35]}$.

When we use the part of the signal behind three-cycle peaks as noise, the signal to noise is not so high any more due to the sidelobes (i.e., in this case,we consider the sidelobes part of the noise.) The signal to noise ratio for binary coded excitation code length 2 (Figure 3.19 (b)) is $115.9 / 12.3=26$; the signal to noise ratio for the backscatter signal (Figure 3.17 (a)) is $0.138 / 0.018=8$. So the encoding gain for binary coded excitation code length 2 is $26 / 8=3.3$. Generally the sidelobes will degrade the axial resolution of ultrasound imaging. This however does not have a great impact in the analysis of speckle patterns as figure 1.11 .

\subsubsection{Impulse response for our binary coded excitation system}

In Section 3.2.1.5, we discuss the impulse response for the coded excitation method. The impulse response for coded excitation method without attenuation and plain attenuation is a $\delta$ function. The encoding results from a quartz reflection are regarded as the combination of the coded excitation method and our experimental system. The coded excitation method without attenuation is a $\delta$ function (Section 3.2.1.5). So the encoding results from the quartz reflection will represent the impulse response of our experimental system. 


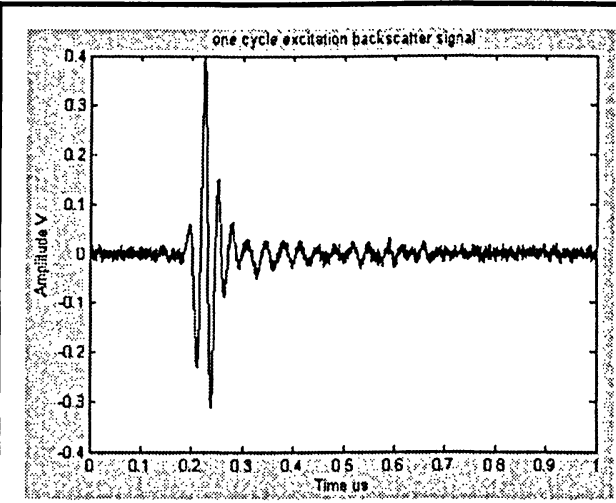

(a.1) quartz reflection from one pulse

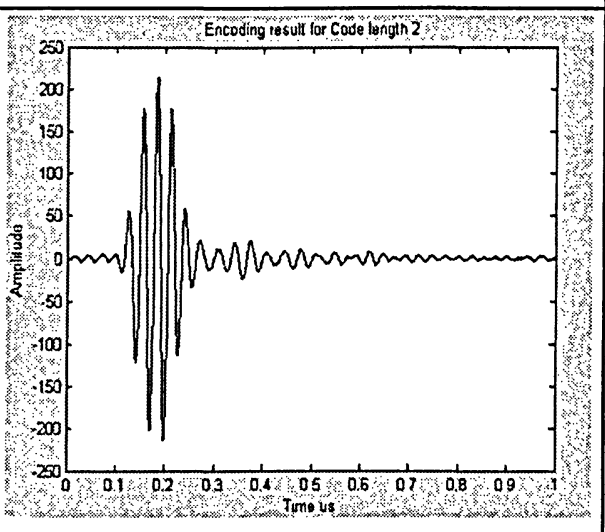

(a.2) Encoding results for code length 2

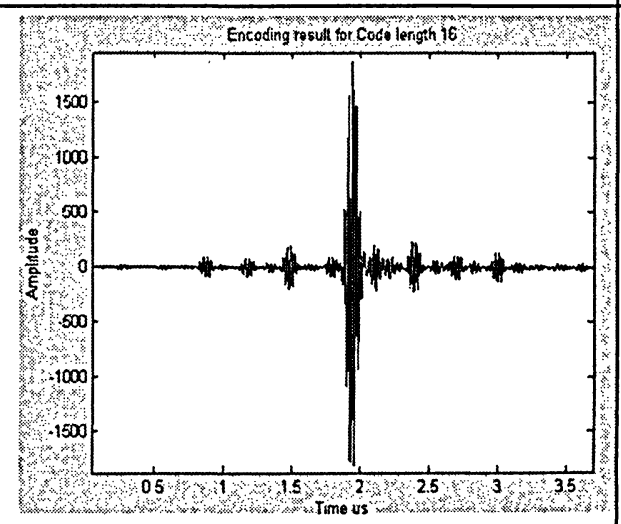

(a.3) Encoding results for code length 16

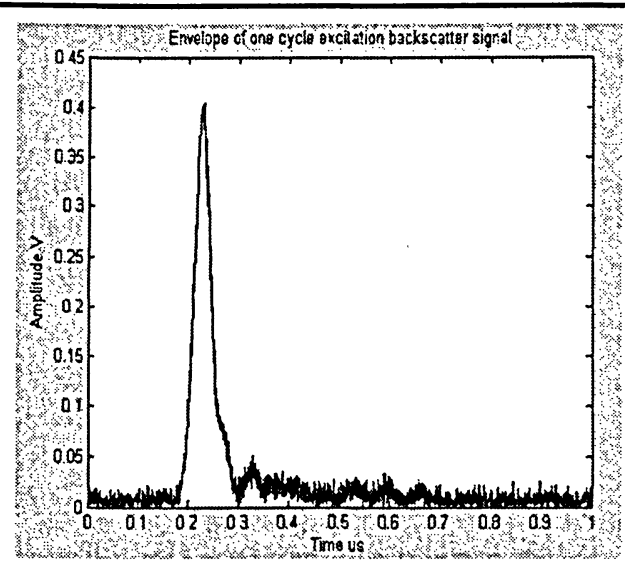

(b.1) The envelope for signal (a.1)

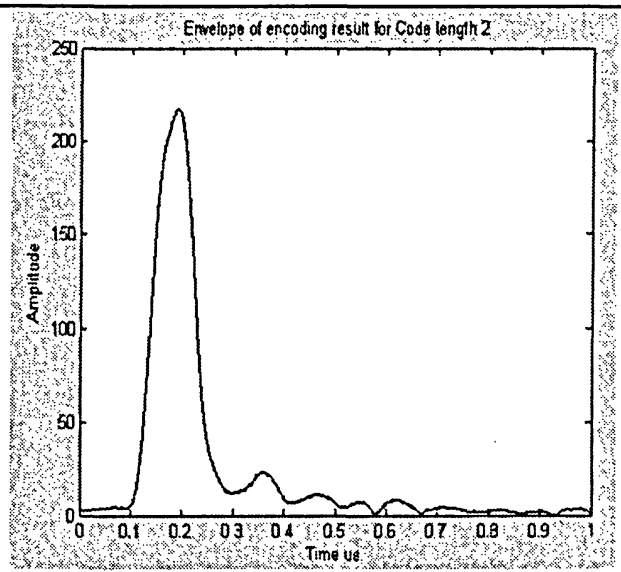

(b.2) The envelope for (a.2)

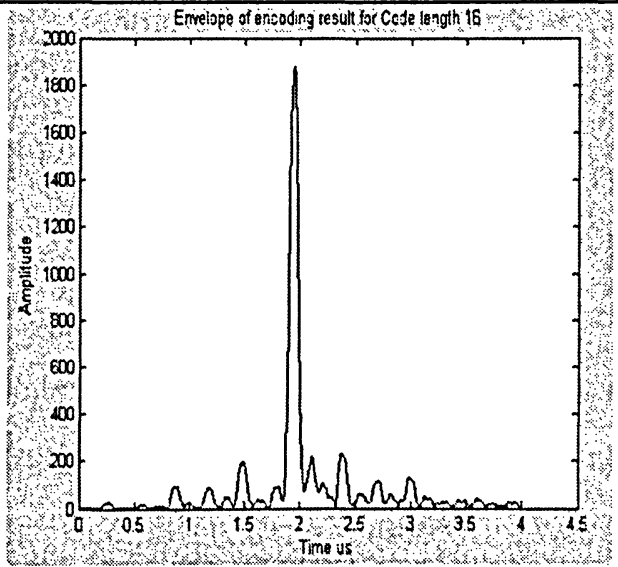

(b.3) The envelope for (a.3)

Figure 3.21 Signals and their Envelopes

When only one pulse is sent to the transducer, the reflected signal from a reflector is 
shown in Figure 3.21 (a1). For a one cycle sine input signal, this output is not a sine. The envelope of one pulses' reflection looks like a $\delta$ function as Figure 3.21 (b1). This output can be regarded as the impulse response for our system, although there are sidelobes and noise in the signal.

In Figures 3.21 (a2) and 3.21 (a3), the encoding results of code length 2 and code length 16 are shown; their corresponding envelopes are shown in Figures 3.21 (b2) and 3.21 (b3). These two envelopes represent the impulse response of the binary coded system for code length 2 and code length 16. In the prototype system we cannot get a perfect waveform mainly due to the inertia of transducer. So for different code lengths, the impulse response is different. From Figures 3.21 (b3) and 3.21 (b2) we find there are more sidelobes for the longer code.

\subsubsection{The Phase Shift Effect}

In Section 2.4.2.2, the problems associated with the phase shift from the movement of the platform were discussed. In this section, an example is given. Assume that there is a 80 sample-time shift between the Golay and complimentary Golay code for the code length 2 . The sample rate in this case is $5 \mathrm{GHz}$, so 90 sample-time means 16 ns shift. The gap is small. In Figure 3.22(a) the encoding results without phase shifting compensation are shown and the encoding results with phase shifting compensation are in Figure 3.22(b). When these two graphs are compared, we find the maximal amplitude for Figure 3.22(b) is 214 and for Figure 3.22(a) is 185 . When comparing the sidelobes, the sidelobes in Figure 2.22(a) are greater than these in Figure 2.22(b). Without phase shifting compensation there is more energy in the sidelobes so that the peak value of signal of is smaller than with phase shifting compensation. 


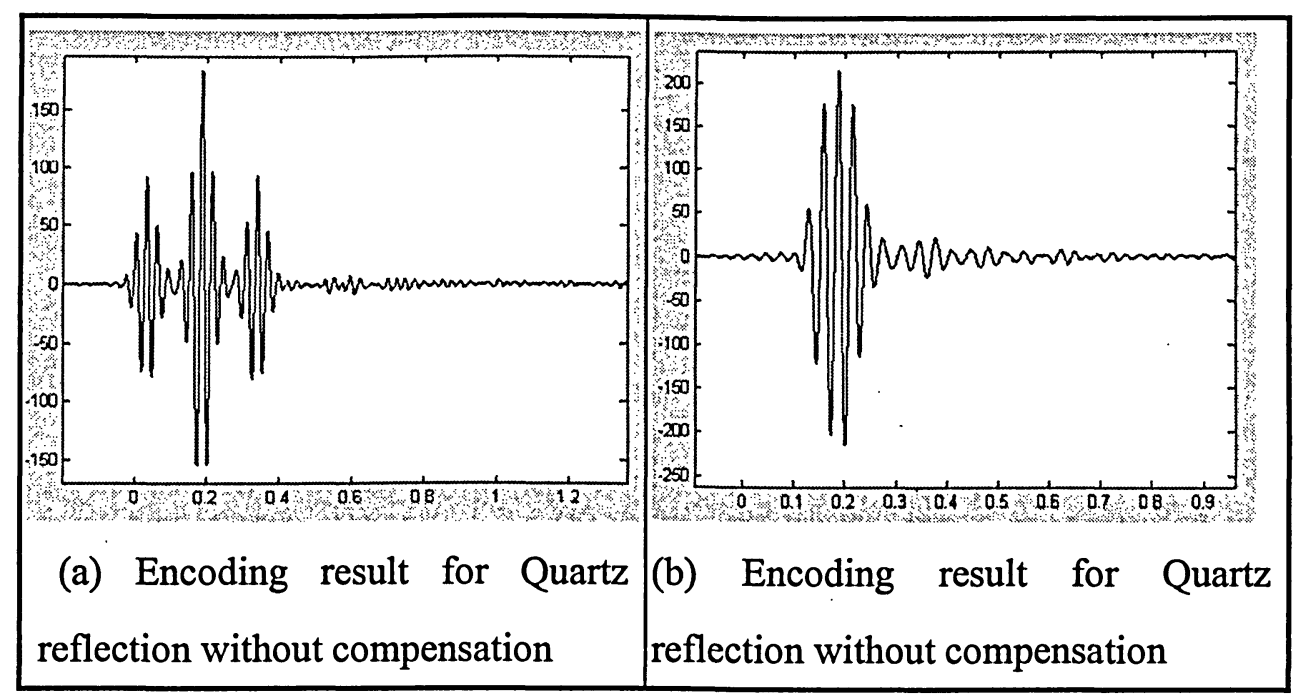

Figure 3.22 Encoding Results for Phase Shift Effect

From these results, we concluded that the binary coded excitation method is very phase sensitive. In section 2.4.2.2 the platform shift problem was solved. If we want to compensate for the phase shift during pulse sequences, the shift distance needs to be controlled. If the shift is more than a half cycle of the ultrasound pulse, it is difficult to compensate for the shift. Another bad effect from the shift compensation is it causes the signal blur if the backscatter signals for Golay code and complimentary code come from different part tissue location and their sum will blur the signal.

\subsubsection{The Effect of Code Length}

In Figure 3.23 the encoding results of the backscatter signal from a quartz reflector using four code lengths are compared. The size and number of sidelobes increase with code length. The amplitude gain increases with code length but is not linearly proportional to code length. 


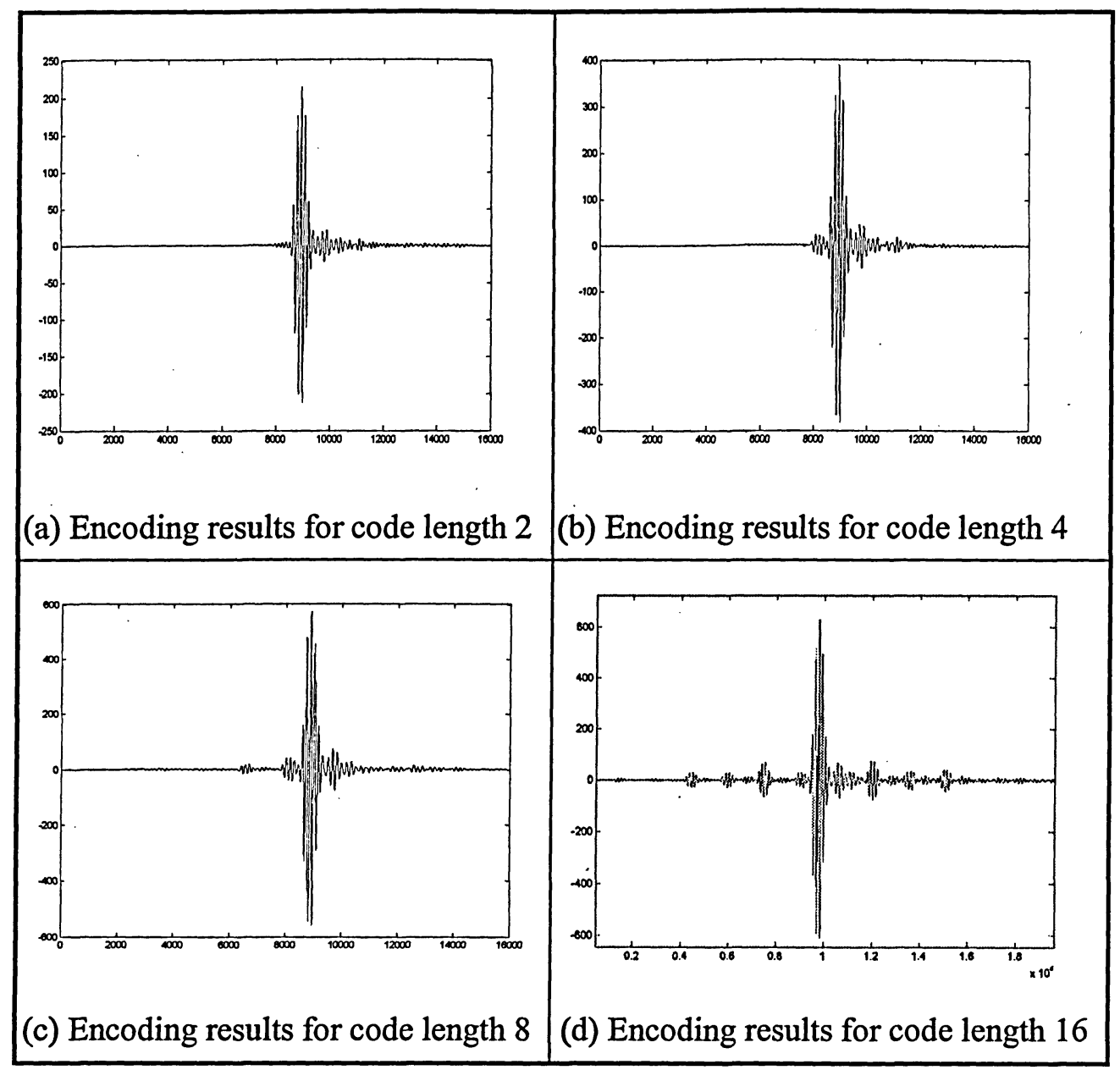

Figure 3.23 The Encoding resultss for Different Code Length

The encoding gains for code length 2 and 4 increase linearly with code length (Figure 3.24). But for code length 8 and 16 the gains do not scale linearly. The main reason is mentioned in Section 2.4.2.1: when the code lengths increases, the amplitude of reflected signal decreases. The second reason is that the sidelobes increase with longer code length. Since more energy of the signal is transferred into the sidelobes, the amplitude is reduced. Compared with Figure 3.15, the encoding gains from experimental reflection attenuation is more than that of theoretical simulation, because experimental results are processed by 3-cycle code with phase reference method and the simulation results are processed by 1 cycle code reference method. 


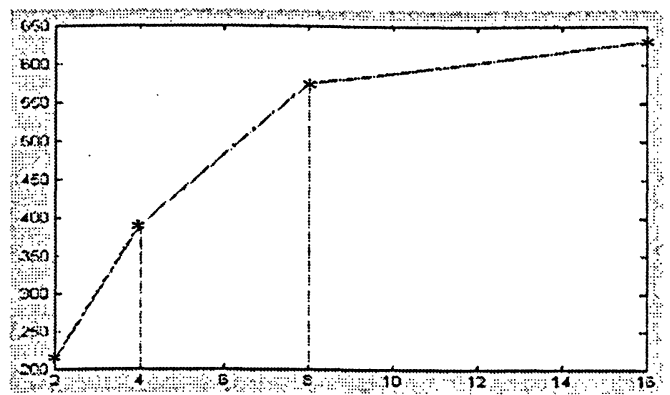

Figure 3.24 Encoding Gains for Four Code Length, X-axis Code Length

\subsubsection{Results from Phantoms}

In this section, binary coded excitation in situations with closely spaced non-resolvable collection of scatterers is examined. First the backscatter signal from a phantom are examined and then from excised liver tissue. The phantom is a good substitute for real tissue for they have stable properties to assess the ultrasound backscatter signal. The phantom in this experiment was made of $4 \%$ agar in water mixed in equal parts with tungsten particles, The tungsten particles are 1.00-1.39 micron in diameter. The speed of sound in tungsten is $5200 \mathrm{~m} / \mathrm{sec}$ and the impedance of tungsten is 101 . The agar is dissolved in boiling water and solidifies when it cools to $37^{\circ} \mathrm{C}$. The beads are in suspension (2,000,000 per ml) and are mixed with the agar when it cools to about $40^{\circ} \mathrm{C}$. The mixture is then poured into the mold to solidify.

The binary coded excitation method is used to increase the signal to noise ratio and thus the penetration depth of high frequency ultrasound. The STD (standard deviation) is used to monitor the signals. The trend of STD for different segments is related to signal distortion. To evaluate this distortion, the signal envelope is calculated. 


\subsubsection{The Effect of Code Length}

Which signal should be used as the reference signal to evaluate the encoding results from binary coded excitation method in tissue? From the analysis of reflection signals and simulation results, we notice the impulse response for binary coded method is like a $\delta$ function. So the output of $\delta$ pulse input may be the answer. For each depth and tissue a cycle of sine signal as our $\delta$ pulse input is sent to transducer and the output backscatter signal from the one cycle sine pulse is captured and stored as our reference to evaluate the encoding results from binary coded excitation method.

Figure 3.25 shows the backscatter signal from a phantom using one cycle sine input. For the same phantom location, Golay code pairs are went to the transducer and the backscatter is stored and analyzed. The results are shown in Figure 3.26. The signals are divided into the 100 segements, each about half encoded code length.

In Figure 3.25(a), the backscatter signal for one pulse, we can use the STD for the signal as our signal power and the STD from the beginning part as noise power. The signal to noise ratio is 10.5 . For three-cycle code with phase processing method (Figure 3.26 (a)), the encoding gain is 52.8. So the encoding SNR gain for binary coded excitation code length 2 is approximately 5 . Because the sidelobes effect does not taken into account, in this results, this result can only used to demonstrate how the binary code excitation improves signal to noise ratio for white noise. Since the sidelobes and the signal are mixed together, it is difficult to evaluate the power of sidelobes in the backscatter signal in Figure 3.25(a). 


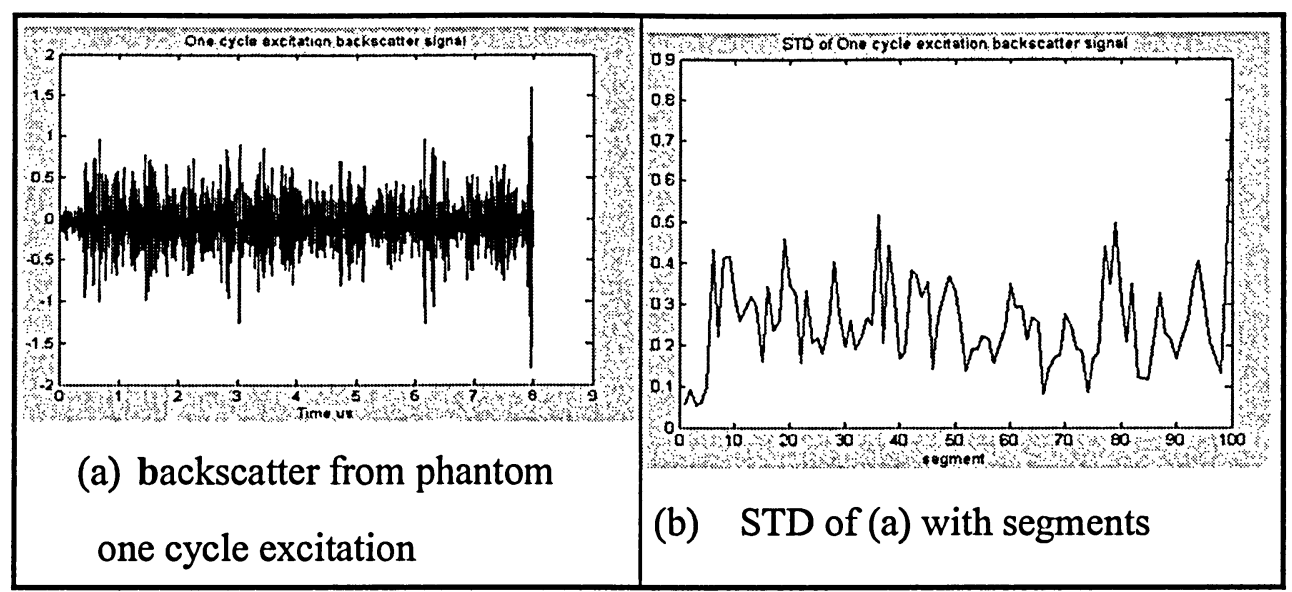

Figure 3.25 One Reference from One Cycle Pulse and Envelope

Comparing Figure 3.26(a1-a4) to Figure 3.25 (a), we find the similarity of the encoding results to the backscatter signal decreases with increasing code length. In Figures 3.25(b), and 3.26(b1-b4), the y-axis is the amplitude of signals and $\mathrm{x}$-axis signal segments. Each segment has 400 sample points. When comparing Figure 3.26(b1-b4) to Figure 3.25 (b), the magnitude of the STD of thebackscatter signal ranges from 0.2 to $0.6 \times 10^{-3}$ and all magnitudes for STD for encoding results ranges from 0.1 to 0.6 . The gain of STD for encoding results is about 1000. If the code length increases, the STD increases. Likely due to frequency dependent attenuation, the gain does not increase linearly with code length. Furthermore, as seen in the simulation, the sidelobes degrade the signal after the decoding compression. 


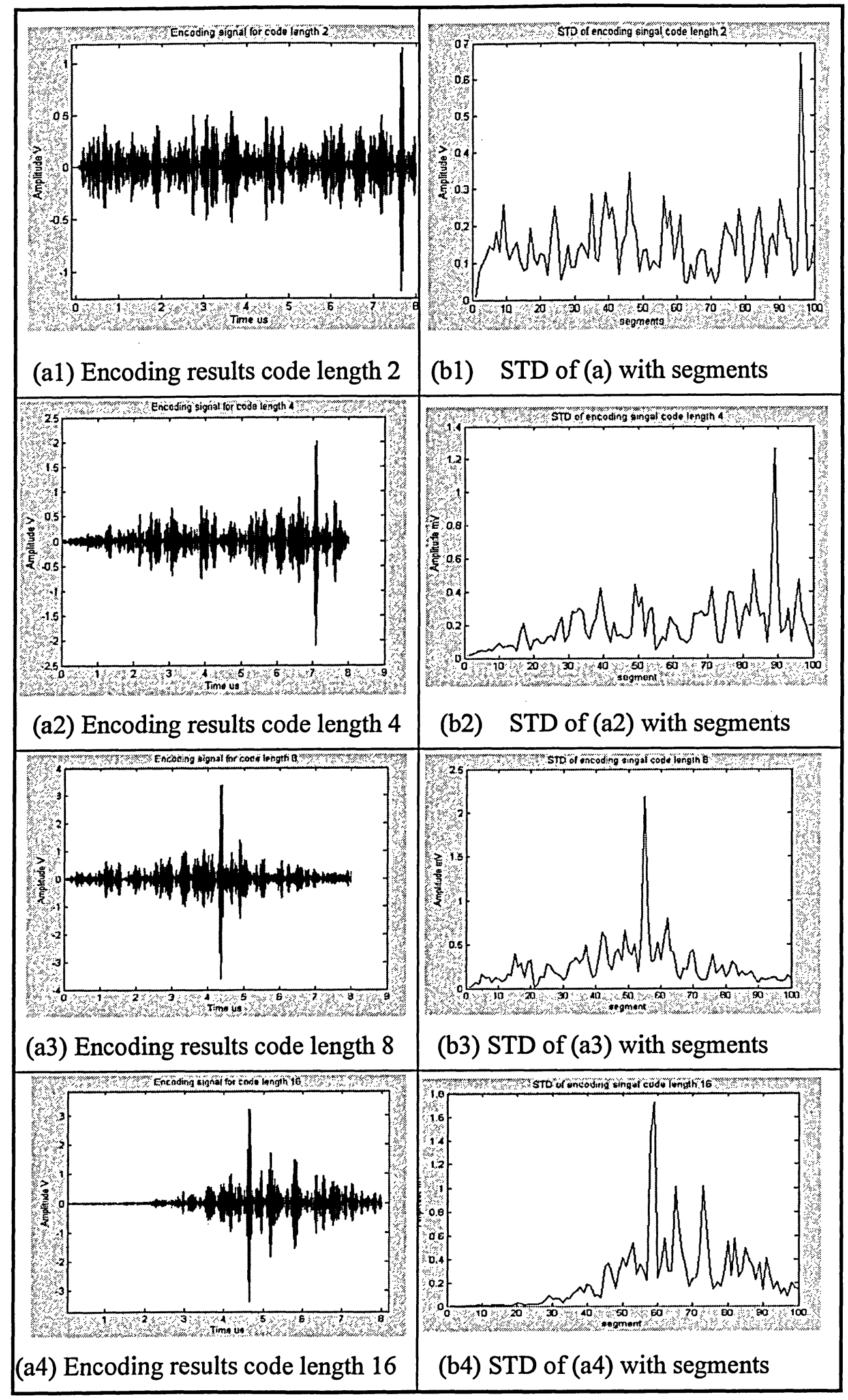

Figure 3.26 Encoding results for Four Code Length (Refer to Figure 3.25) (Note Change in Scales) 
Comparing Figure 3.26(a1-a4) to Figure 3.25 (a), the similarity of encoding results to the original one cycle excitation backscatter signal decreases with increasing code length. The gain of STD for encoding results is about 1000 times. If the code length increases, the STD increases.

When comparing Figures 3.26(b1-b4) to Figure 3.25 (b), the reason for the feature that the peaks of the backscatter signal move to the left of the figure with increasing code length is unknown.

\subsubsection{The Effect of Depth}

In this section, the backscatter signals from the phantom are compared with the transducer focused at different depths. The focus of transducer lies in three different depths $0.3 \mathrm{~mm}, 0.5 \mathrm{~mm}$, and $0.7 \mathrm{~mm}$ under the surface of phantom and the ultrasound backscatter signals are shown in Figures 3.27, 3.28 and 3.29. In all these three figures, the reference backscatter signal from one pulse input is shown in (a). STDs of these backscatter signals for different segments are shown in (b). The encoding results using Golay code of length 2 and STD for encoding results are shown in (a1) and (b1). 


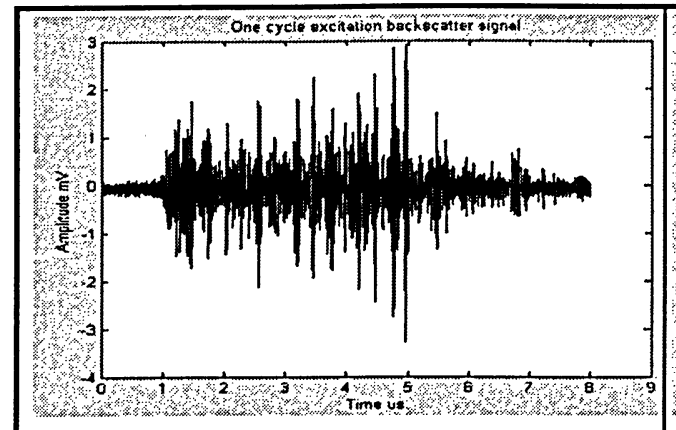

(a) one cycle excitation backscatter

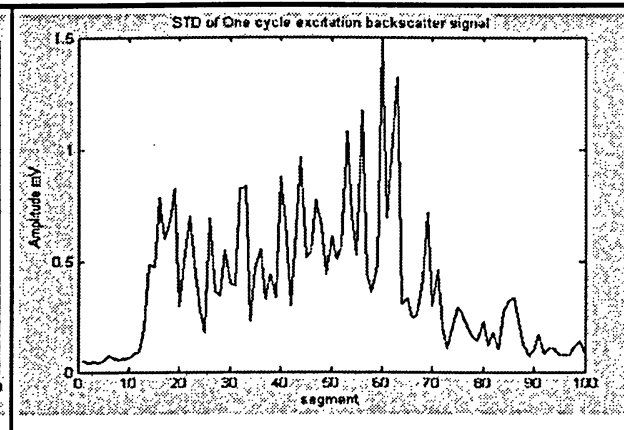

(b) STD of (a) with segments

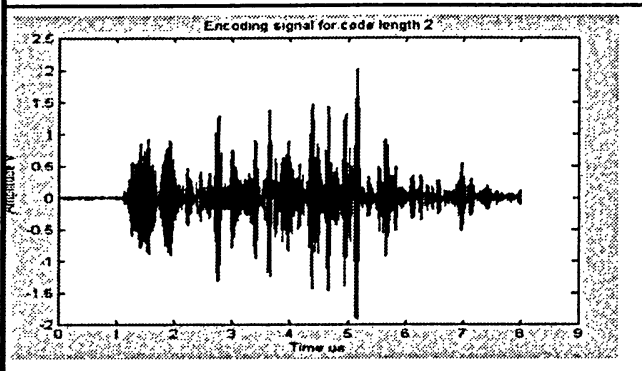

(a1) Encoding results

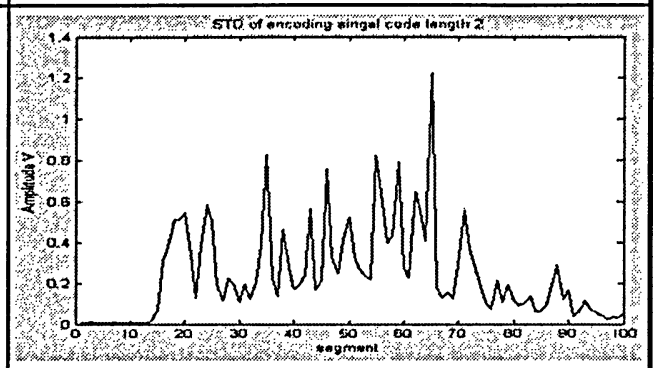

(b1) STD of (a1) with segments

Figure 3.27 Backscatter Signal and Encoding results and Their STD with segments and Focus at $0.3 \mathrm{~mm}$ below Surface

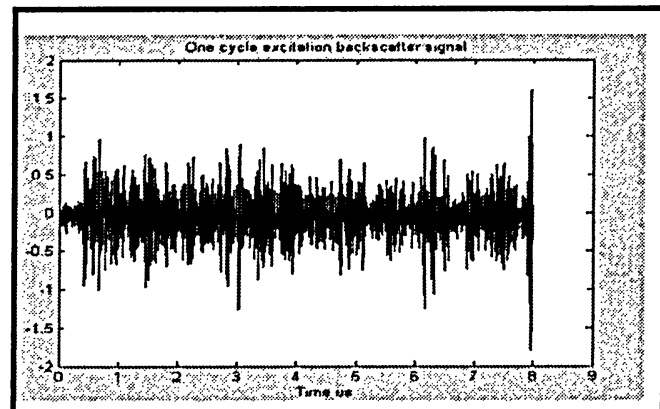

(a) one cycle excitation backscatter

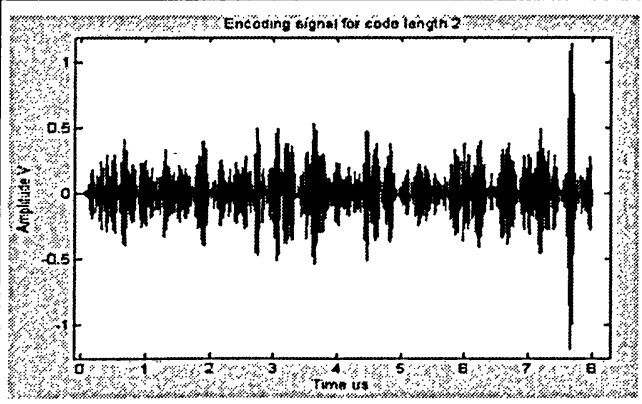

(a1) Encoding results

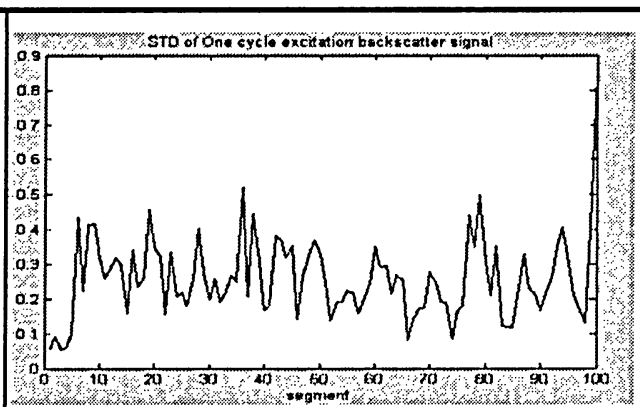

(b) STD of (a) with segments

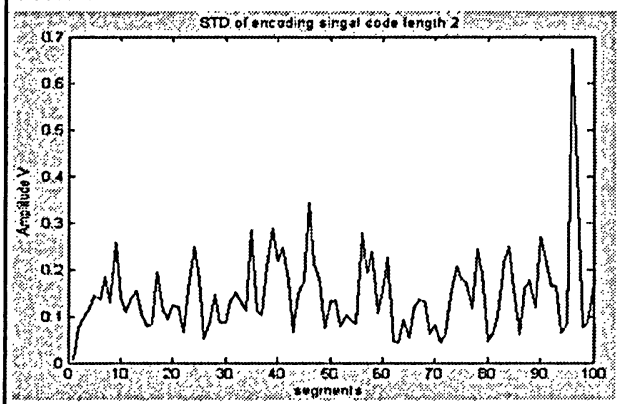

(b1) STD of (a1) with segments

Figure 3.28 Backscatter Signal and Encoding results and Their STD with segments and Transducer Focus at $0.5 \mathrm{~mm}$ below Surface 


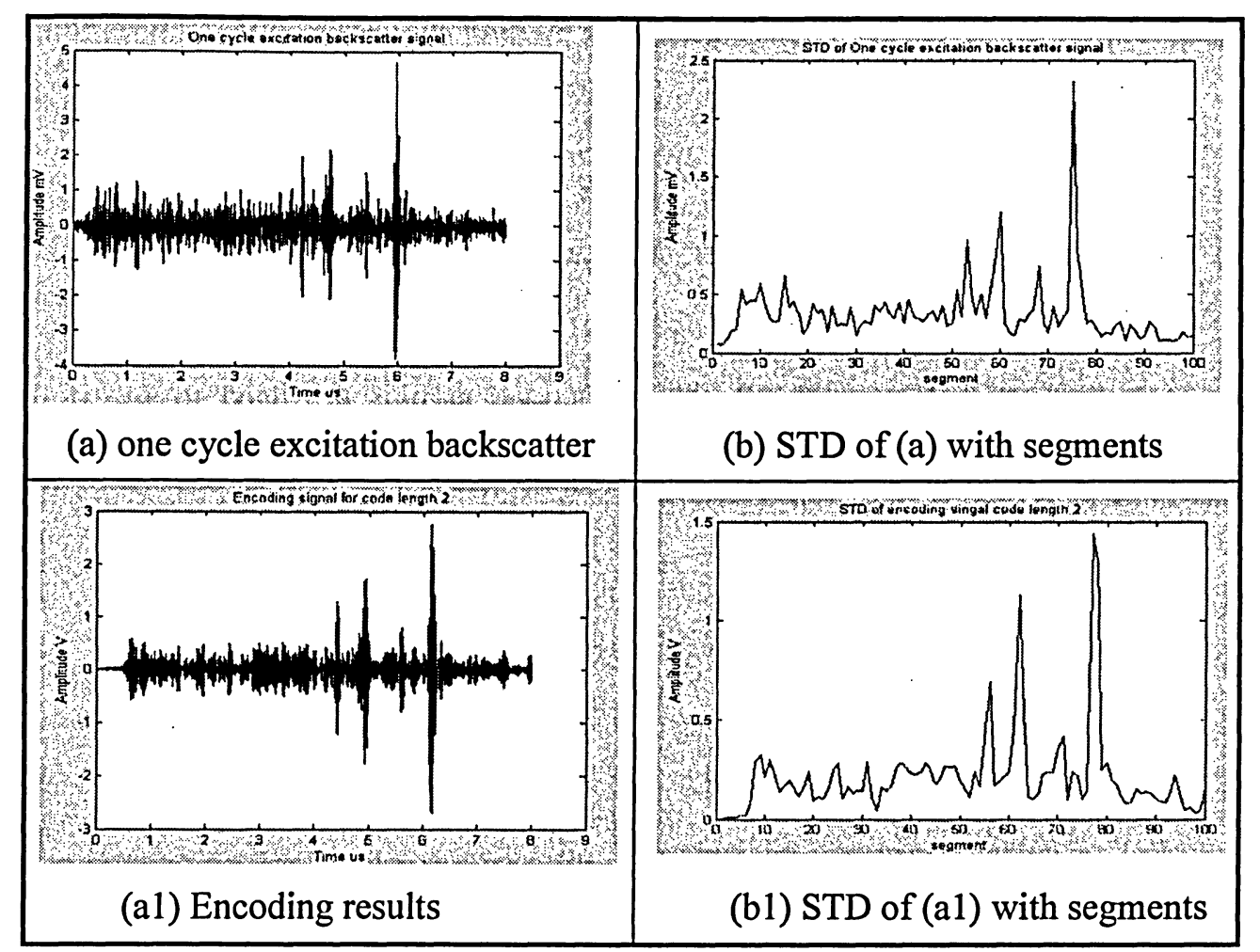

Figure 3.29 Backscatter Signal and Encoding resultss and Their STD with segments with Focus at $0.7 \mathrm{~mm}$ below Surface

The STD of the encoding results with segments for code length 2 (Figure 3.27(b1), Figure 3.28(b1) and Figure 3.29(b1)) and the STD of corresponding backscatter signal from one cycle excitation pulse (Figure 3.27(b), Figure 3.28(b) and Figure 3.29(b)) look similiar. The amplitude for the former ranges from 0.2 to $0.6 \times 10^{-3} \mathrm{~V}$ and the amplitude for latter one range from 0.1 to $0.8 \mathrm{~V}$. The gains of the STD for all three depths signal are 1000 times. Therefore, When code length 2 and when the backscatter signal is several times greater than noise, the binary coded excitation method is suitable for high frequency ultrasound for different depths.

\subsubsection{Critical Depth for Our System}

The critical depth can be defined as the depth at which the amplitude of the 
backscatter signals is equal to noise. If the binary coded method can improve the signal at the critical depth, it means this method can improve the penetration depth.

In this experiment one cycle of sine signal is sent to the transducer and the backscatter signal is noted. Then the gain of the power amplifier is decreased so that the total energy sent into transducer decreases. When the signal is almost submersed into the noise (Figure 3.30), Then encoded signals are used to see if the SNR increases.

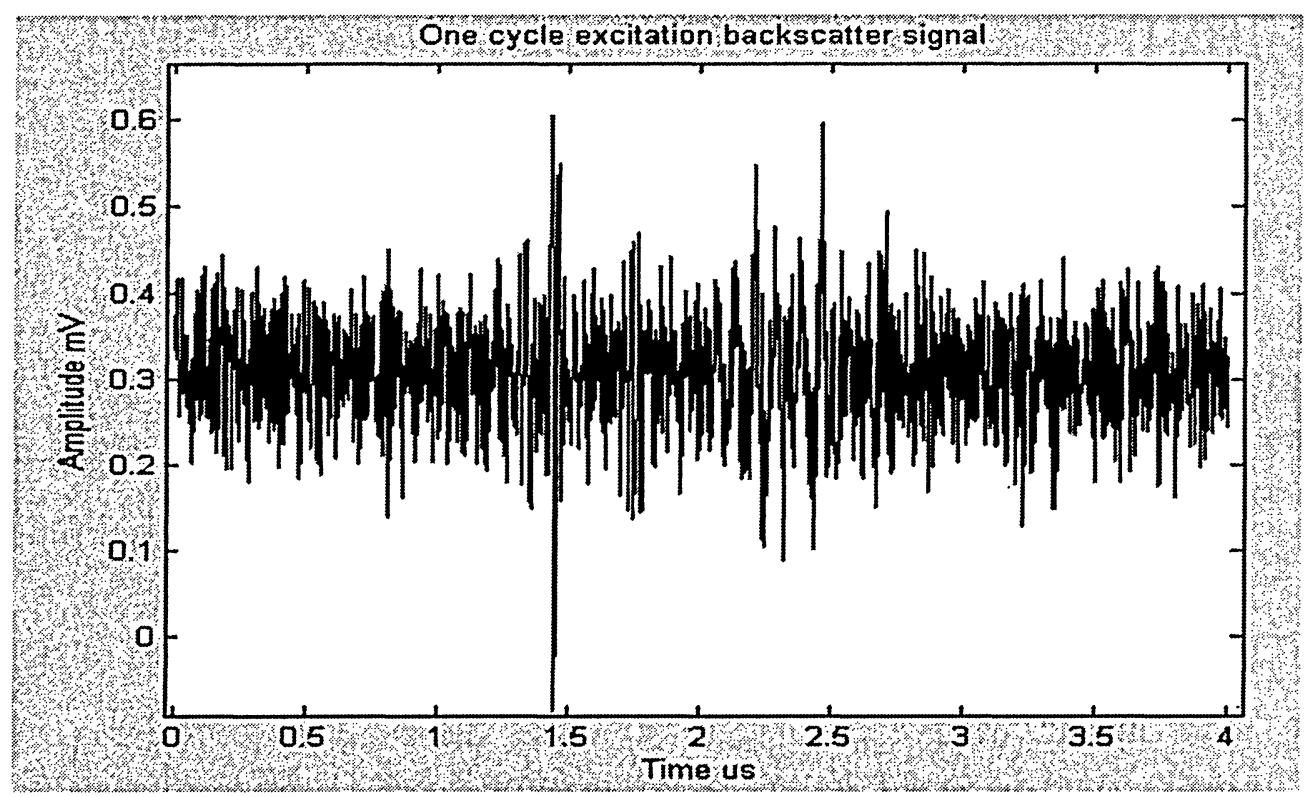

Figure 3.30 Reference Signal at Critical Depth

In Figure 3.31 (a1-a4) the backscatter signals for Golay codes with code length 2, 4, 8 and 16 are shown at the critical depth for the same location and the same phantom. Comparing the signals in these four figures with the backscatter signal of one cycle pulse input in Figure 3.30, the amplitude of backscatter signal for Golay codes increases slightly because more energy is sent into transducer with a longer code length. With longer code lengths, the maximal amplitude of the backscatter signals moves to the right of the figure. This phenomenon is the same as that the focus of backscatter signals for longer Golay code changes deep as we had discussed in Section 2.4.2.1. 


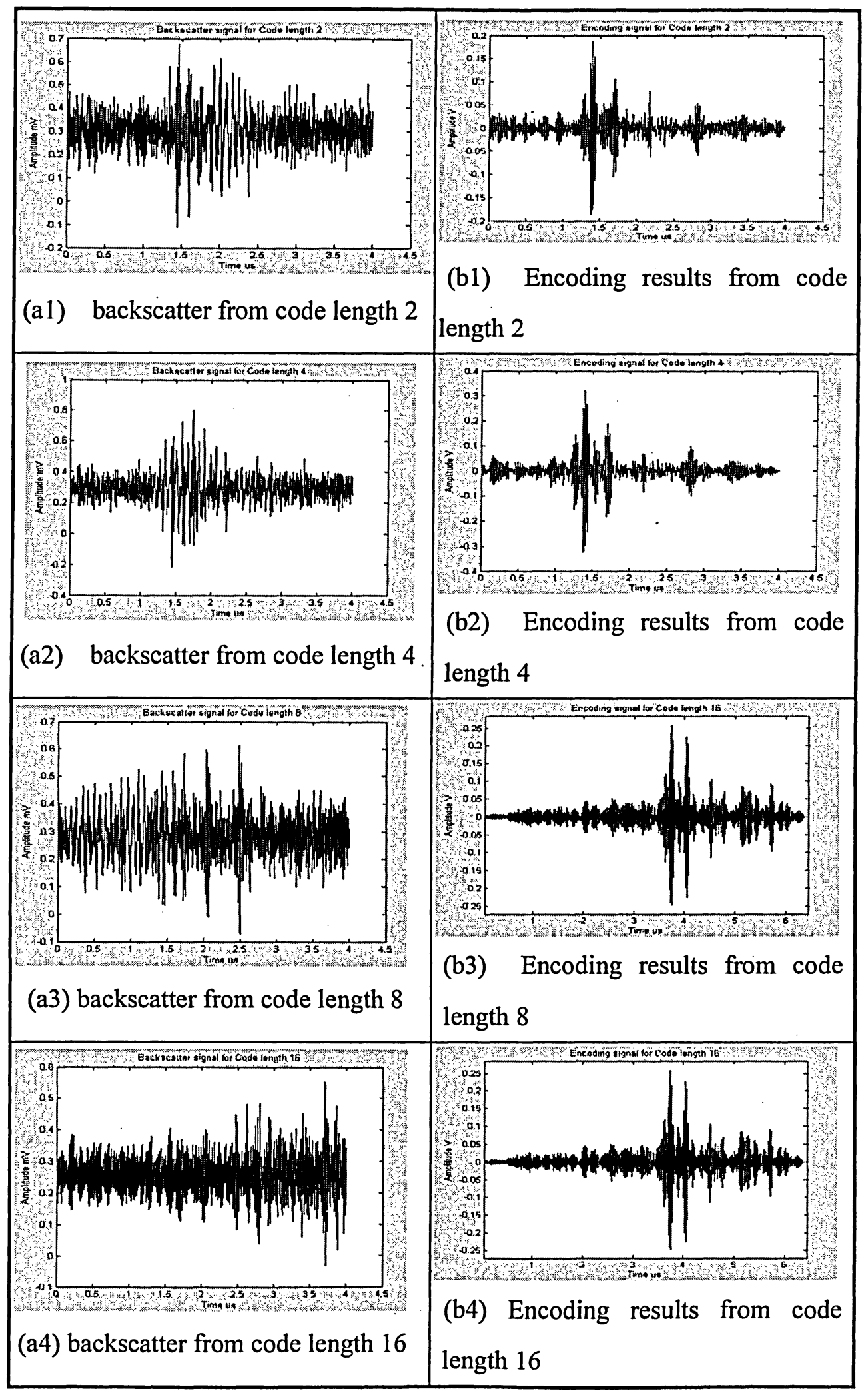

Figure 3.31 Backscatter Signal and Encoding results for Different Code length

(Note the Difference in Scales) 
In Figure 3.31(b1-b4) the encoding results for Golay codes in code length 2, 4, 8 and 16 are shown. The shapes of the four encoding results are similar to each other and all of them have two peak values as the single pulse backscatter in Figure 3.30. The amplitude increases with longer code lengths, but the amplitude decreases for the code length 16. Comparing Figures 3.24 and 3.32, the gains of amplitude at critical depth is almost the same as those from reflection except code length 16.

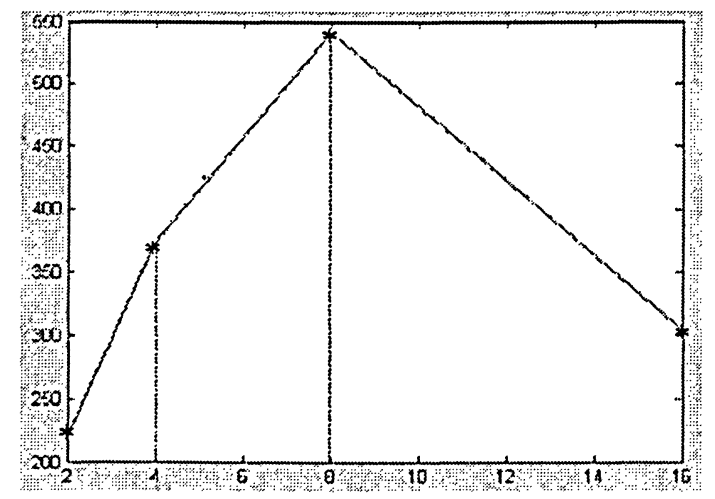

Figure 3.32 The Code Length and Encoding Gain

In order to determine whether coded excitation increases signal from noise, a noise reference measurement was made. The noise reference means the noise from our coded excitation system is processed by three-cycle code with phase processing method as same as we process the encoded signal (first this noise cross-correlates separately with a Golay reference and its complimentary Golay reference, and then they are added) as in Figure 3.33(b). When we power on all the instruments in our system but do not send any signal into the transducer, the output from signal amplifier is just white noise as in Figure 3.33(a). The amplitude of noise is in $3 \mathrm{mV}$.

When we compare Figure 3.33 (a) with Figure 3.30, the amplitude of Figure 3.33(a) ranges from $-0.25 \mathrm{mV}$ to $-0.5 \times 10 \mathrm{mV}$ and the most of signal in Figure 3.30 ranges from 2.0 to $4 \mathrm{mV}$. So they are in almost same range. But when we compare Figure 3.33 (b) with Figure 3.31 (a1-a4)(the encoding results), the amplitude in Figure 
3.33(b) ranges between \pm 0.02 and the signal is uniform; but in Figure 3.31(a1-a4), the peak amplitude of signals ranges $0.15-0.4 \mathrm{~V}$ and the signal is not uniform. So the peak values in Figure 3.31 (a1-a4) are signal increased by binary coded excitation method.

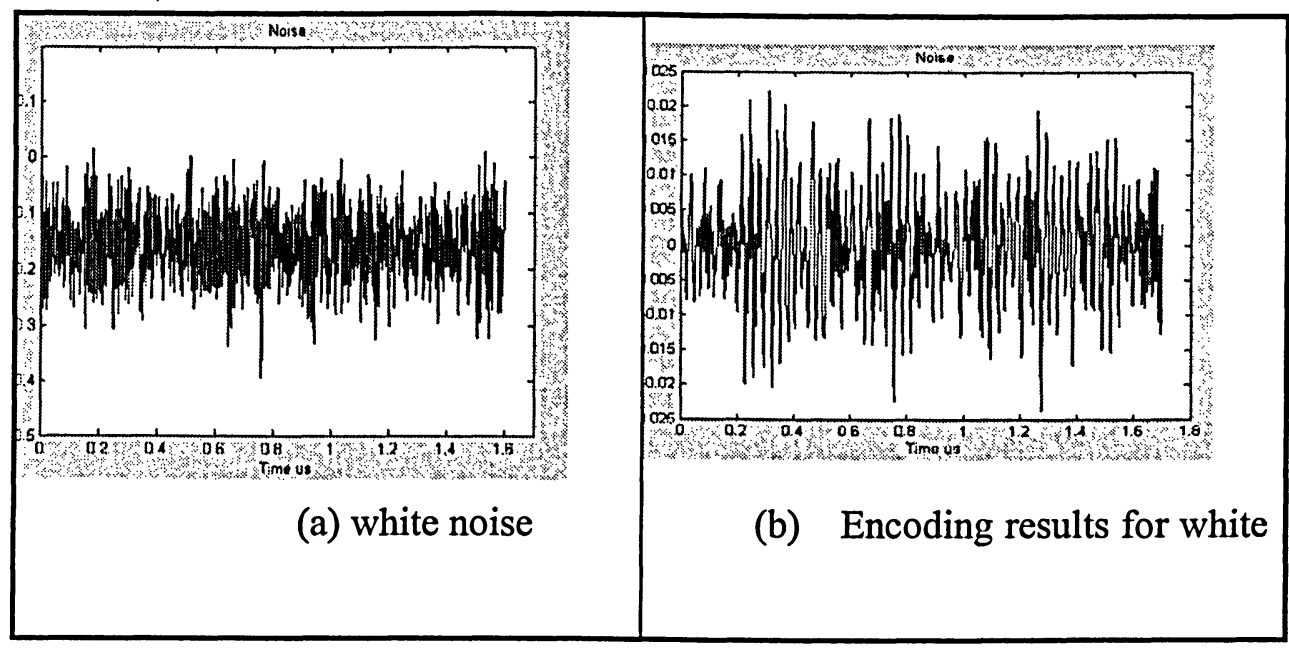

Figure 3.33 White Noise and Its Encoding resultss

In Sections 3.3.4.1, 3.3.4.2, and 3.3.5, we discuss some results based on the backscatter signals from the phantom. From these results, the binary coded excitation still increases the signal energy, and the envelopes of encoding results of coded signal are similar to that from one pulse backscatter signal. Compared with the simulation results, at longer code lengths we cannot realize the anticipated gains. In the last chapter of our results, we will present one example from bovine liver.

\subsection{Backscatter Signal from Real Tissue}

In this section results from real tissue are analyzed. In Figure 3.34, the backscatter signal from bovine liver is shown. This signal is generated when one cycle sine pulse is sent into the transducer., The backscatter signal from real tissue only appears around the focal region of the transducer but the backscatter signals from the phantom are significant even outside the focal zone as in Figures 3.25(a), 
3.27(a), 3.28(a), and 3.29(a)), because the phantoms have a stronger backscatter coefficient than the tissues we used.

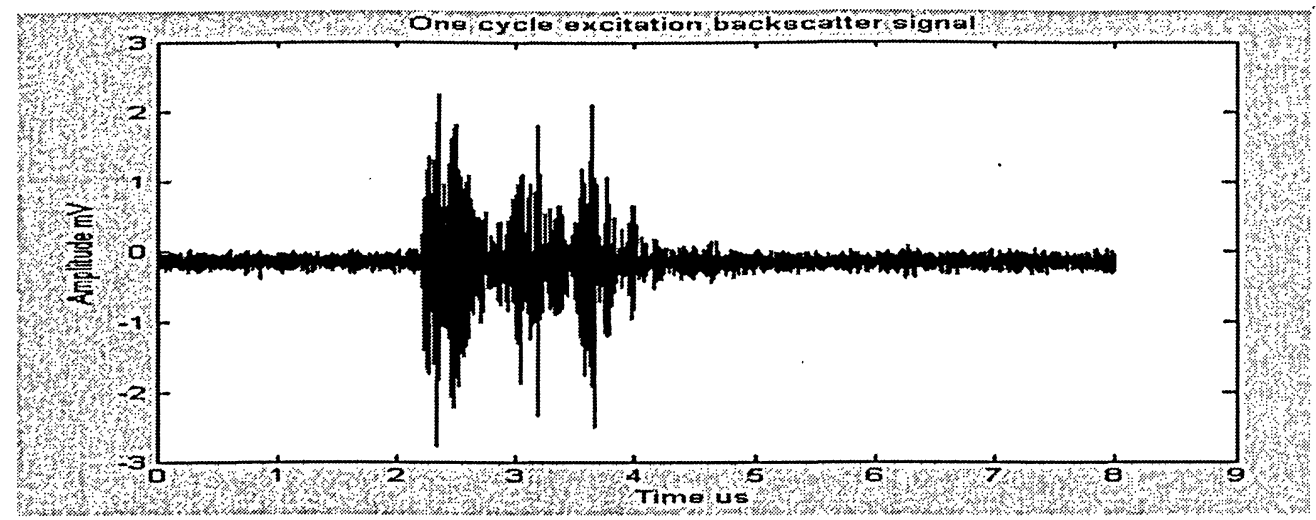

Figure 3.34 Backscatter Signal from Bovine Liver

\subsubsection{Different processing methods}

In Section 3.4.1, the three-cycle code with phase, reflection and one cycle code, and concluded were compared, and demonstrated three-cycle code with phase gives the largest gain. In Figure 3.35, the backscatter from liver using different encoding techniques is shown. The green color signal is the encoding results of three-cycle code with phase; the red signal in (a) is the encoding results from reflection reference; the red signal in (b) is the encoding results from 1 cycle code with phase. The gain in the three-cycle code with phase method is almost twice as the other two methods. 


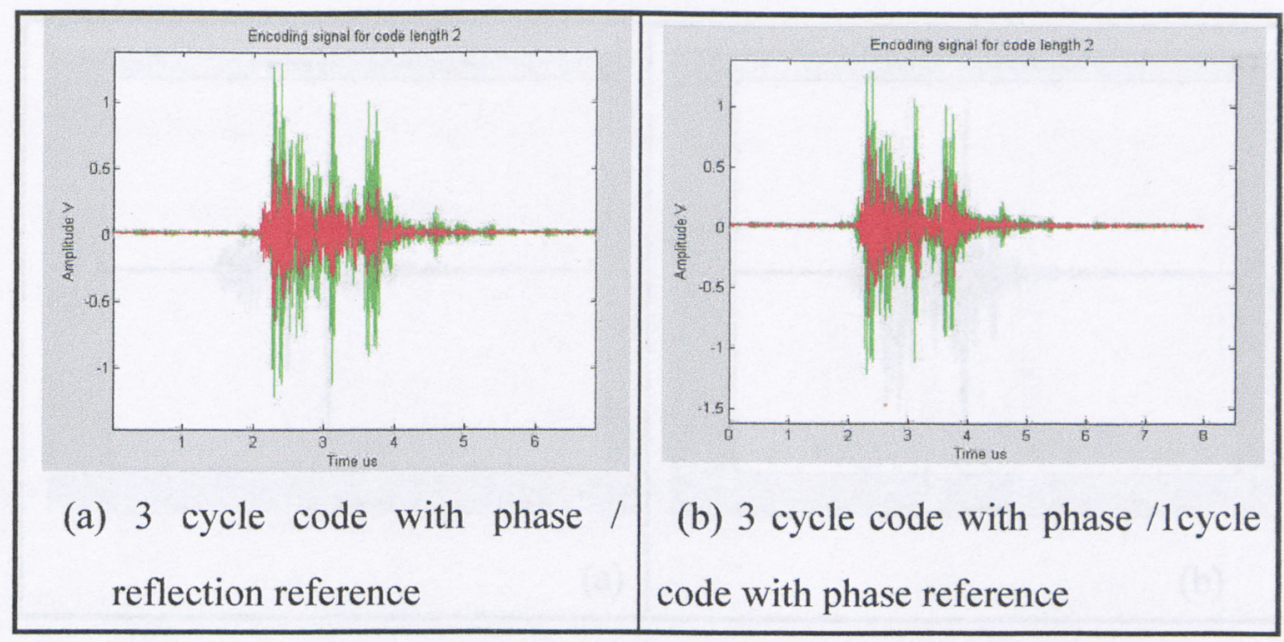

Figure 3.35 Backscatter Signal from Liver Tissue Using Different Methods (Code Length 2)

\subsubsection{Phase Shift}

In Section 3.3.4.1, the effect of phase shift on the reflected signals is discussed. The phase shift between the backscatter signals of the Golay code and complimentary code will increase the sidelobes of the encoding results and lower the maximal amplitude. In Figure 3.36, backscatter signals for liver tissue with and without phase compensation are presented. In Figure 3.36(a), the signal is not like the backscatter signal in Figure 3.34 even though it was taken from the same location. But in Figure 3.36(b), in which a phase shift correction was applied, the encoding results look more similar to the signal in Figure 3.34. In summary the phase shift has an important effect on the encoding results of binary coded excitation method, even though the time shift is very small between the Golay and complimentary Golay code pairs. For example, in Figure 3.36 the signals are from code length 4 and the shift for Golay code and complimentary Golay code is $8 \mathrm{~ns}$, or equivalently $12 \mu \mathrm{m}$. 


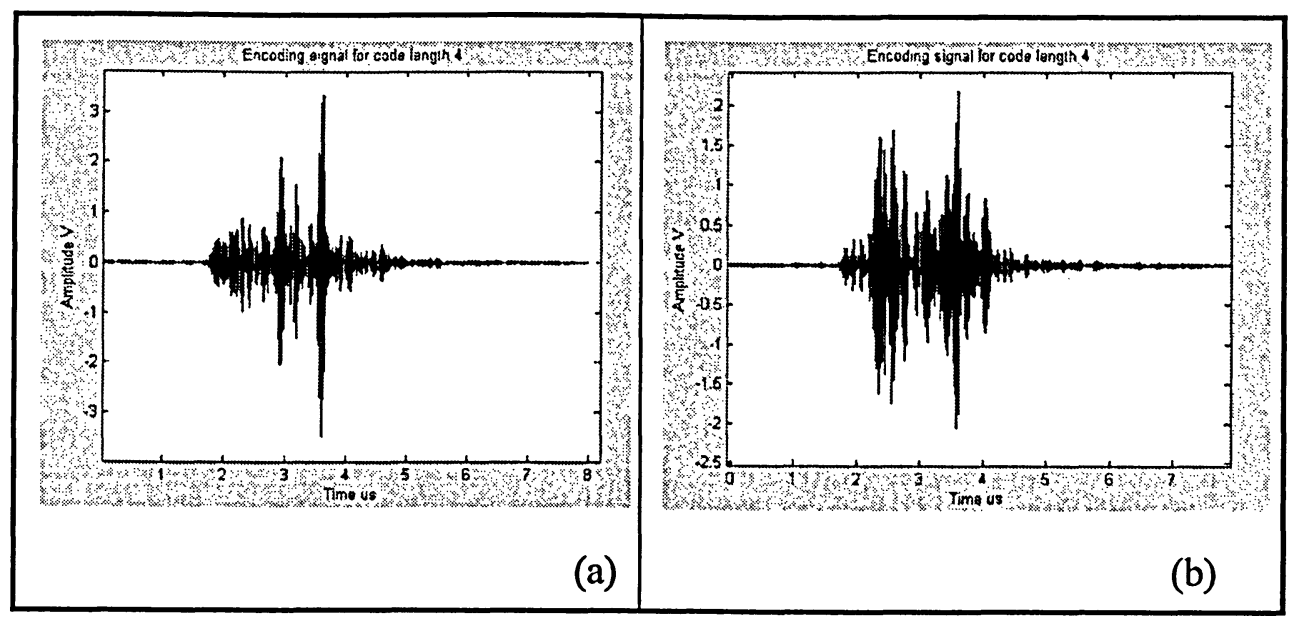

Figure 3.36 Backscatter from Bovina Liver using Coded Excitation (a)

Without Phase Compensation (b) With Compensation

\subsubsection{Similarity of Signals}

In Sections 3.3.4.1 and 3.3.4.2, we use trends in the STD of backscatter to represent the similarity of original backscatter signal and the results from binary coded excitation. A greater similarity of these two signals means the distortion of signals due to sidelobes is less. In this section an envelope method is used to evaluate the similarity between original backscatter signal and the encoding results from coded excitation. In this method we cross correlate both signal envelopes; if the cross correlation result is more similar to a $\delta$ function, the similarity of these two signals is high. And the distortion of encoding results is smaller.

In Figure 3.37 (a) is the envelop for reference signal in Figure 3.34; the envelop of encoding results from code length 2 (as Figure 3.35 green color signal) is Figure 3.37 part (b); the envelope of encoding results from code length 4 is in Figure 3.37 part (c); in part (d) the dot line is the cross correlation of envelopes of reference and encoding results from code length 4 , the solid line is the cross correlation of envelops of reference and encoding results from code length 2. After normalization to these two results, they are shown as Figure 3.37 (d). 


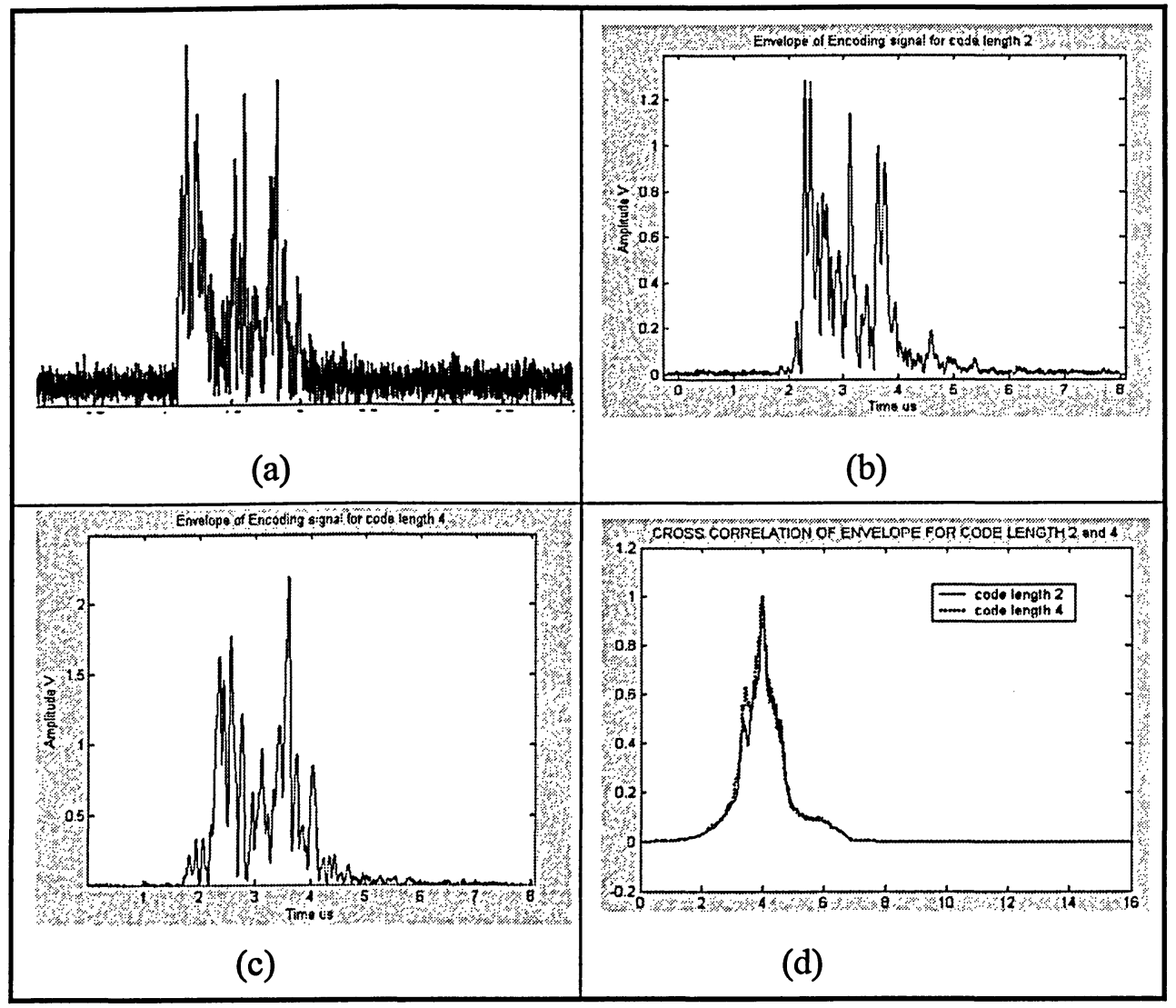

Figure 3.37 Envelope Method to Evaluate Signal Similarity of backscatter signals

From Figure 3.37(d) we find both cross correlation signals approach a $\delta$ function. The similarity of the signal from code length 2 to the original is a little higher than that from code length 4 as expected.

\subsubsection{The Gain in Signal to Noise Ratio}

The signal to noise ratio is the most important parameter to evaluate the quality of coded excitation method. Why we just discuss this important topic so late? From our simulations and experimental results we find the sidelobes increase with depth and longer code length. The sidelobes have a strong effect on the encoding results 
of binary coded excitation methods. The noise in binary coded excitation includes two parts: normal noise and sidelobe noise. Unfortunately the sidelobes of encoding results are mixed with the signal. It is difficult to separate sidelobes from signal.

In this section A method to evaluate both parts of noise so that we obtain the gain of signal to noise ratio in binary coded excitation method. General noise of our system is regarded as white noise. As show in Figure 3.34 the beginning part of backscatter signal before signal appears is regarded as noise of our system. So the STD of this part of signal is regarded as general noise for our lab system and the value is about $5.6 \times 10^{-5}$ and STD for signal is about $7.4 \times 10^{-4}$ So the signal to noise ratio is about $7.4 \times 10^{-4} / 5.6 \times 10^{-5}=13$; and corresponding part in Figure 3.35(a) is regard as general noise in encoding results and the value is about 0.0072 and STD of signal in encoding results is $\mathbf{0 . 6 6 3}$. So the signal to noise in encoding results is about $0.663 / 0.0072=92$. If we just consider general white noise, the signal to noise ratio for encoding results in code length increases $92 / 13=7$ times about 8 dB.

A method is used to estimate the energy of sidelobes. Noise and signal are mixed together in encoding results. From the quartz reflector data as Figure 3.25, it is possible to evaluate the energy of signals and sidelobes. The ratio of energy of signals to the energy of sidelobes is regarded as another SNR ratio. The square of the area of huge impulse is over to the square of the area of sidelobes (as Figure 3.23 (b)) to evaluate the signal to sidelobes noise. The area of huge pulse is $1.6405 \times 10^{7}$ unit $^{2} \times$ ns and the area for sidelobes is $2.422 \times 10^{5}$ unit $^{2} \times$ ns. So the signal to noise ratio is 67 for sidelobes noise.

In code length 2, sidelobes from reflection signal have a little effect in SNR. For the SNR for sidelobe noise is 67 , which is greater than, the SNR gain for white noise 7 times. 


\section{Chapter 4 Conclusions and Future Work}

\subsection{Conclusions}

\subsubsection{Feasibility of Binary Coded Excitation Method}

The general structure of the coded excitation system is developed for high frequency ultrasound imaging is based on the conventional ultrasound system. An arbitrary waveform generator was chosen to replace general pulser generator in a conventional ultrasound system. A wide band (1-500MHz) analog power amplifier was selected to amplify the signal sent to transducer and all the signal channels of conventional ultrasound system were redesigned. Two problems in our binary coded excitation system developed were discussed in Chapter 2. After several problems were solved, coded backscatter signals in phantoms and real tissue were collected. After analysis of these signals, some reasonable results are reached and gains of $12 \mathrm{~dB}$ were achieved. Although a lot of work is required to improve the system, this system demonstrates the feasibility of binary coded excitation for high frequency ultrasound and provides unique insights to the future.

The design of the system for binary coded excitation accumulates the energy of signals from different time slots together. After binary coded excitation method is used in our high frequency ultrasound systems, whether in simulation or experimental systems, we realized gains in amplitude, STD and signal to noise ratio of the signals. Furthermore, the backscatter signals from the pulser and the signals from coded excitation method were similar. So the binary coded excitation method is a way to improve the signal to noise ratio of high frequency ultrasound signal and increase the penetration depth.

The gain of signal amplitude is almost linearly proportional to sample rate (section 
3.2.1.4 and section 3.2.2.4). When the sample rate is high, the gain is high. Because it is equal to the sum of the amplitude for all sample points, when the number of sample points increases, the sum increases. So this method is not good to evaluate the effect of the binary coded excitation method. But this method is very useful in sparse spacing model in the simulations and the reflection signals in experiment to evaluate if their results are reasonable or not. When the sample rate is the same, the amplitude gain still reflects the gain in signal to noise ratio.

Gain in the STD of signals, similar to amplitude gain, is directly related to sampling rate. The STD if the signals is used because the amplitude gain is not good to evaluate the backscatter signal from tissue or theoretical models with closely spaced scatterers. The gain in STD is used to estimate the gain with and without coded excitation. This method mixes the power of signal and noise together. If the signal is much greater than the noise, this method is used to evaluate the power gain of signals. When the SNR is low, this method is not appropriate.

The gain in signal to noise is the best method to evaluate the binary coded excitation method. In my thesis we discuss the gain of signal to noise briefly. because coded excitation cause sidelobe noise and this sidelobe noise is mixed with signal. How to effectively estimate the sidelobe noise of coded excitation method is a prerequisite to calculate the signal to noise ratio correctly. It is difficult to evaluate sidelobe in backscatter signal of real tissue from coded excitation. However, sidelobe noise reduces the axial resolution of the system, but still produces useful signals for analysis on for the area of research in our lab is not detrimental.

\subsubsection{The Mechanism of the Binary Coded Excitation Method}

How does the binary coded excitation method work in improving the high 
frequency ultrasound signal? Binary coded excitation extends the signal duration so that the total energy in the signals from coded excitation method is greater than that from a conventional pulser (as FM coded excitation does.). Using the sparse spacing model in the simulation or quartz reflection signals in the experiments, longer signals are reflected at the same scatterer or same reflection point, so that we can "accumulate" such a long signal together. This is the reason why we can "accumulate" all the signals for backscatter signal from tissue and closely space models. In tissue when the backscatter coefficient of a volume is huge, the sum of these signals with longer coded excitation is huge; when the backscatter coefficient of a scatter is small, the sum will be small. The sum of these longer signals denotes the backscatter property of a scatter as one pulser backscatter signal does. When comparing our encoding results from binary coded excitation with backscatter signal of one pulser input, the similarity of both signals verifies the conclusion that just made.

In Sections 3.3.3 and 3.4.4, I find that the binary coded excitation method can improve the signal to noise ratio. How does binary coded excitation method improve the signal to noise? First of all the sum of the longer signal alone indeed increases the amplitude of the signal. Thus the total energy in the signal is amplified. The second reason is that noise is averaged during the cross correlation operation. When signals are added together by the cross correlation operation, the noise mixed into signal is also added. But because noise is uncorrelated, the gain in noise is not as large as that for the signal. The third reason is due to effective suppression of sidelobe noise by Golay code pairs. Our results from shorter code length 2 and 4 the Golay code pairs can reduce almost all the sidelobe noise in plain attenuation model. In FD attenuation tissue this method still works, but it does not reduce all the sidelobes any more. 


\subsubsection{The Effect of Code Length}

When the code length of the coded excitation is longer, more energy is contained in the signal. Therefore, one might expect that the gain of energy will increase linearly with code length. In the simulations, whether sparse or dense spacing and whether plain attenuation or FD attenuation, we find that the gain of the encoded signals from binary coded excitation method always increases with code length.

The theoretically predicted gain with increased code length was not achieved experimentally. Only when the code lengths are 2 and 4, does the gain increase linearly with code length. When code lengths are 8 or 16 , the gain increase is much smaller than predicted. The reason for this is discussed in Section 2.4.2.1. The amplitudes of signals for code length 8 and 16 are attenuated. For code length 16 , the amplitudes of the code sequence even look like a coned and maximal amplitude is only half of those of code length 2 or code length 4 . Further more the focus of the transducer for code length 16 moves $1 \mu$ s deeper. It is not clear why this should be so. How the transducer behaves when excited by the long sequence is not understood, and there are no published data on this. However, it should be a topic of future work.

\subsubsection{Phase Shift Sensitivity}

The phase sensitivity is used to denote the effect on the encoding gain for a phase shift between Golay and Complementary Golay code pairs due to sample motion. When a phase shift sensitivity is high, the same phase shift will have a larger effect on the results; when a phase shift sensitivity is low, the same phase will have a less effect. We find experimentally that the sensitivity of the technique to phase is large., Even a 40 to $80 \mathrm{~ns}$ time shift between the Golay code and complimentary Golay code has a significant effect on the encoding results (In Section 3.3.4.1) . For example, in Section 2.4.2.2, if there is ns-order time delay 
between the Golay and complementary code sequences, the amplitude of encoding results is smaller and the sidelobes increases.

Because the binary coded excitation is so sensitive to the phase shift, this method is not suitable when there is considerable motion. 40 to $80 \mathrm{~ns}$. second are equivalent to motions of $60 \mu \mathrm{m}$. The minimal time between Golay codes pairs sent is 1 to 2 minutes due to delays in the arbitrary waveform generator and time domain averaging used to increase the SNR. In the encoding results we can see the effect even the target moves 60 micrometer in 1 to 2 minutes. In the future if new binary coded excitation system can send and receive Golay codes pairs in a shorter time period, it is possible to use this method to slowly move targets.

The code with phase reference method is better than that of the code reference method. In Section 3.3.3, the encoding gain using code with phase reference is almost doubled than gain using the code reference method. Comparing all three processing methods of code with phase reference, code reference and reflection reference, the code with phase processing is the best one to decode the binary coded excitation.

In Section 4.2.2 during analysis of binary coded excitation method we assumed that the encoding result signals are the sum of coded modulated signal form the same position or scatterer. This conclusion is correct for the signals from reflection in experiment and sparse spacing model in simulation. For the backscatter signals from tissue and density spacing model in simulation, this conclusion does not work any more. Because the distances between scatterers are so small that all the backscatter signals are mixed together, it is impossible to add all the signals from the same scatterer to generate the encoding results. The backscatter signals from scatterers nearby will contribute to the encoding results. The binary coded excitation method sacrifices the resolution to get gain in energy. 


\subsubsection{Impulse Response and Similarity of Signals}

In Section 3.3.3 we define the impulse response for coded excitation, for plain attenuation as a perfect $\delta$ function. This is helpful to explain why binary coded excitation works. We use the envelopes of reflected signals for different code length to represent the impulse response of that Golay code pairs. These impulse responses are worse with increasing code length. In Section 3.4.3 the similarity of encoding resultss to the backscatter signal from unit pulser is defined. It also shows that the similarity of signals worsens with code length. The impulse response of the binary coded excitation method is directly related to the similarity of signals. For this reason we can analyze the quality of different codes by just the analysis of impulse response of those codes in later sections.

In Section 3.3.4, we compare the impulse response for two different code lengths. The impulse responses worsen with code length, as more energy is shifted to the sidelobes. In the binary coded excitation system, only code length 2 and 4 works well; the encoding results for code length 8 and 16 do not work well. The reason for this is not clear, but is related to the time between pulses.

\subsection{Future Work}

This thesis has laid the groundwork for future work on coded excitation for high frequency ultrasound. Many aspects need to be more thoroughly investigated. The next paragraphs explain some of these.

In Section 2.4.2 some interesting phenomena are shown. When the code lengths are 8 and 16, there is attenuation in the reflected signals from a quartz plate. For code length 16 , the amplitudes of the code sequence for different bits resemble a cone and the maximal amplitude is only half of those of code length 2 or 4 . More experiments are required to figure out what is the exact reason. If we find the 
interpretation for these phenomena, it is possible to use longer codes in our binary coded excitation system, as longer code sends more energy into the targets that should lead to greater improvement in the SNR.

In Section 3.4.4 calculations of the increase in SNR using binary coded excitation are made. How to evaluate the sidelobes noise for backscatter signals from tissues is not clear enough to reach a conclusion on gains in the SNR. How cross correlation operation works on FD attenuation of biomedical ultrasound signals, especially in high frequency band range, is worth more research. Only after further understanding of this topic, one may have the ability to take advantage of binary coded excitation method.

In a lot of work on the FM coded excitation such as Jian Shen's, Matthew O' Donnell's, Pai-Chi Li's and Thanassis X.Misaridis' has been done, the optimal filters are discussed to process the FM coded excitation to reduce the sidelobes noise. In Section 3.3.3 I find the sidelobes still affect our results using the binary coded excitation method. Some further processing methods were implemented to suppress the sidelobe noise to improve the signal to noise ratio.

In our binary coded excitation method we changed the signal generator to arbitrary waveform generator. The power amplifier is changed into a linear wideband one. All the signal channels are redesigned to fit our new requirements. But the core of ultrasound system, or the transducer, is a conventional one. In Section 2.2.1.3 I find that, even if just one pulse is sent to this transducer, several cycles of signal are sent out due to the inertia of the transducer. These vibrations cause more sidelobe noise. For good binary coded excitation ultrasound systems, special transducer is required to have less inertia and capability to send a long code pairs with less distortion.

In Section 4.2.4 we discuss the problem of phase shift. A lot of work is required to 
find how the resolution and phase shift compensation are effected by varied phase sensitivity. Some methods and parameters are required to evaluate the relations between phase sensitivity, resolution and phase shift compensation. In the end we can design our binary coded excitation system to meet our requirement on phase shift and correct for this. 


\section{Appendix A System Design}

In this section we discuss how to build up a binary coded excitation high frequency ultrasound system. First all the components we select for our system are listed. Then the methods to use these components and make them match are introduced. In the end the connection of all system is presented. I made several small circuits for our system. All these circuits are explained in this section in details.

\section{A1.1 Arbitrary Waveform Generator ${ }^{[1]}$}

Arbitrary waveform generator is an important component in our binary coded excitation system. It is used to generate binary modulated Golay coded signals. The main features of the waveform generator are:

- The maximal clock frequency: $2.6 \mathrm{G}$

- D/A convert accuracy: 8 bit

- Waveform memory: 8.1M-word

- Two arbitrary marker outputs

- Five waveform editors

- An Ethernet port for using NFS or FTP link

- GPIB interface for remotely controlling or transforming waveform from external source.

- A port for connecting 101 or 106 type keyboard

- Inter clock for setting up the current date and time

The interface of arbitrary signal generator is shown in Figure A1.1.

${ }^{[1]}$ Sources Tektronix Inc. "AWG 610 arbitrary waveform generator user manual" 


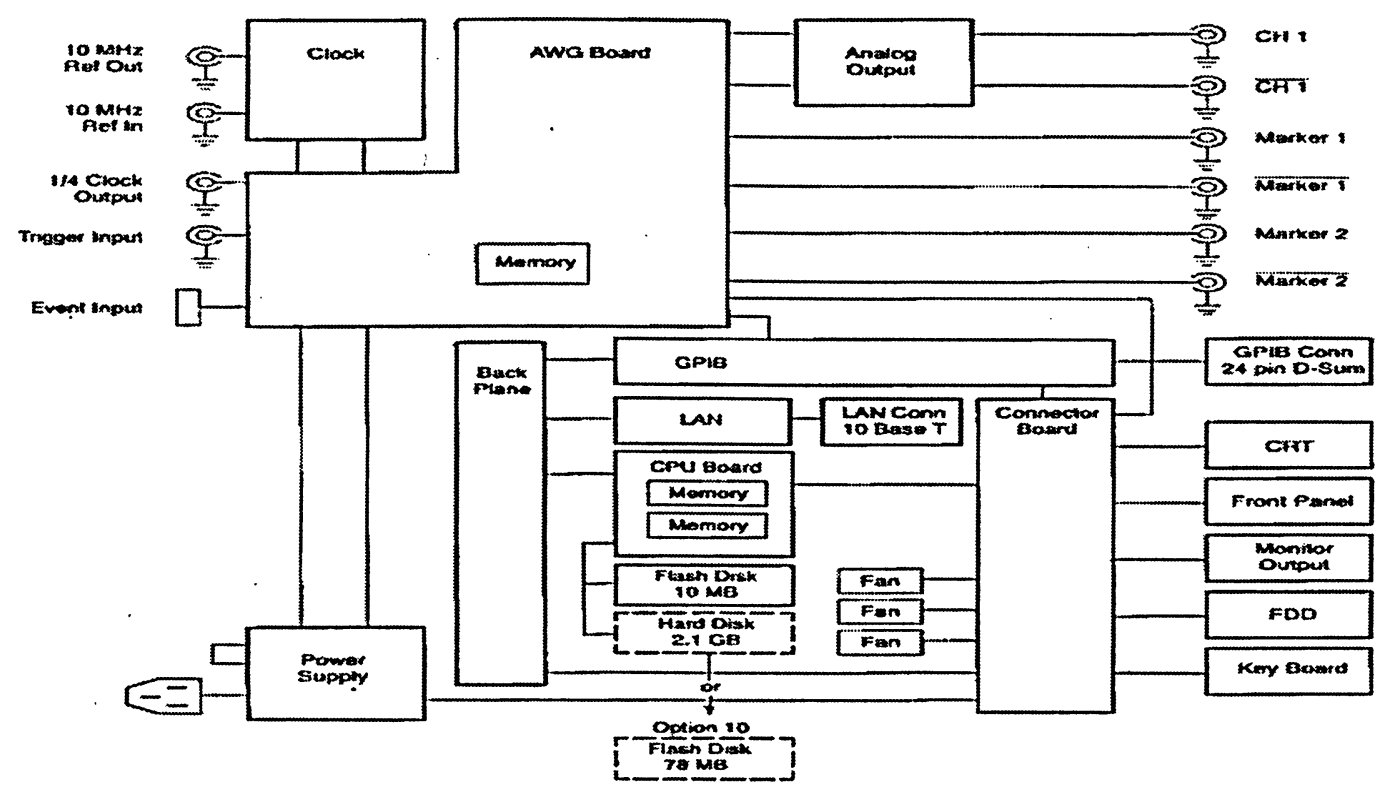

Figure A1.1 The Interface of Arbitrary Signal Generator

The analog circuit structure of arbitrary signal generator is shown in Figure A1.2.

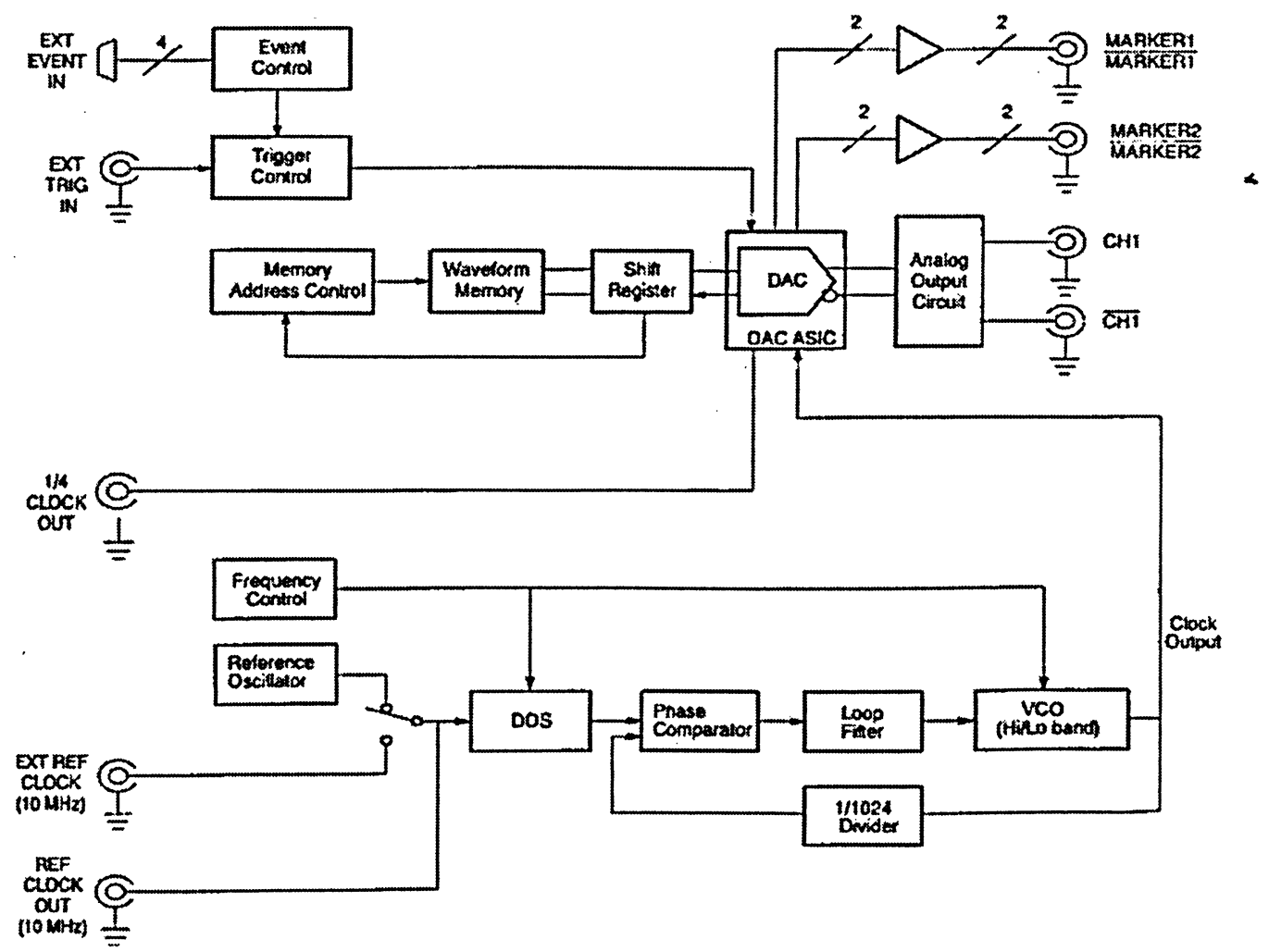

Figure A1.2 The Structure of Arbitrary Signal Generator 
This structure is important to the understanding of how the signal generator works. The clock oscillator can be set to either the internal or external reference clock source by using the SETUP menu. If the external clock source is selected, the reference signal connected to the $10 \mathrm{MHz} \mathrm{REF}$ in connector on the rear panel will be used. The internal clock is connected to the reference clock oscillator, which uses the direct digital synthesis (DDS). The waveform memory has 8 bits for waveform data and 2 bits for per channel for markers, a total length of $8.1 \mathrm{M}$ points. The length of the waveform data must be chosen from 512 to $8.1 \mathrm{M}$ points, and it must be a multiple of 8 . There are three RUN modes including Triggered, Gated, and Enhanced for selection. The RUN mode represents the way to send the signal out. In our case, the signal needs to be sent out continuously and repeatedly. Thus, the Enhanced mode is selected.

Five editors may be used to draw the waveform in this instrument. These are the Graphical waveform editor, the pattern editor, the table editor, the equation editor, and the sequence editor. After the waveform is drawn, the waveform is loaded into the memory. Through the RUN mode, the waveform is sent out as the signal. From these procedures we know editing waveform and generating the signal will be completed in separate time. In this machine there is a Quick Editor. We can edit the waveform in memory directly. It means we can send out our edit on line.

The Graphical editor, the Equation editor and the Sequence editor were used in this project. Implementation of these three editors is explained in the following:

1) The Graphical editor. First select the Graphical editor. Then set the clock frequency of the signal and the size of the waveform. As the blank window appears on the screen, move the cursor to the area to be edited. The OPERATION must be selected to finish the drawing. The OPERATION has three types; the drawing operation, editing operation and mathematical operation. In the drawing 
operation, sine, triangle, square, ramp, or noise generators can be selected to generate the desired waveform. In the editing operation, replace, insert, copy, paste, cut, horizontal shift, horizontal rotate, vertical shift, expand, vertical scale, horizontal invert, or vertical invert option can be selected. In the mathematical operation, absolute, square, cube, square root, normalize, differential, integral, add, sub, multiply compare, convolution, correlation, or digital filter can be selected,

Table A1.1 The Predefined Variables

\begin{tabular}{|l|l|}
\hline Function & Description \\
\hline Clock & Set the clock frequency for signal \\
\hline Fname.clock & $\begin{array}{l}\text { Returns the clock frequency of the special file name, this variable can not be } \\
\text { used as variable in a waveform equation }\end{array}$ \\
\hline Pi & The Ludolphian number $\pi=3.1415926$ \\
\hline Point & $\begin{array}{l}\text { The current point number value, starting at } 0 \text {, read only, only useable within and } \\
\text { equation. }\end{array}$ \\
\hline Scale & $\begin{array}{l}\text { The current scale value that increase } 0 \text { to } 1, \text { read only, only useable within and } \\
\text { equation. }\end{array}$ \\
\hline Size & Set the current waveform record length \\
\hline Fname.size & $\begin{array}{l}\text { Returns the waveform length of the special file name. This variable can not be } \\
\text { used as variable in a waveform equation }\end{array}$ \\
\hline Time & $\begin{array}{l}\text { The current scale value that increase } 0 \text { to } 1, \text { read only, only useable within and } \\
\text { equation. }\end{array}$ \\
\hline
\end{tabular}

2) The Equation editor. This editor can be used to transform an equation into the waveform signal. A text file describing the equation with certain format is first created. A compiler is then used to compile the text file into the corresponding waveform. Similar to the Graphical editor, the Equation editor has all the functions that the graphical editor supports. The difference is that in the Equation 
editor the function is expressed in KEYWORDs. For example, conv() denotes the convolution operation. There are three types of KEYWORDs: the mathematical keywords, the command keywords and predefined variable. The command keywords include not only all the editing operations and drawing operations in the Graphical editor, such as $\sin ($ ), sqr() and eopy(), but also several new features such as IF (control statement), FOR (loop control) and join(), which concatenates two files into one file. The predefined variables are summarized in the Table A1.1.

The sequence editor is used to create a sequence file. A sequence file is a list of waveform file names that the instrument will produce. Additional parameters, such as repeat count, event triggering, and conditional jumps allow to generate large and complex output waveform.

In this project, the waveform is generated as described in the following steps. A single cycle of sine waveform is first generated using the Graphical editor. The inverse sine signal is then obtained. The frequency of the waveform is required to be set for the sine wave. For example, if the clock chosen is $2.6 \mathrm{G}$, the period of $40 \mathrm{MHz}$ signal is $0.025 \mu \mathrm{s}$. The length of the signal is $2600 \times 0.025=65$. If the oscilloscope is used to measure the width of the signal at 65 points, the period of signal is $0.034 \mu \mathrm{s}$.

Two modulated signals, a sine signal and an inverse sine signal are shown in Figure A1.3. A gap between the two signals is used to restore the transducer. In the same figure we find the reflection signal of one cycle sine input from a quartz plate lasts for a long time but the large amplitude lasts only three-period of center frequency. So the next pulse should send out some time delay to avoid these three-period of large signal. In experiment we use 5 periods of center frequency as a gap to restore the transducer and avoid the three large amplitude signals. The time for 45 points is $0.025 \mu$ s. Therefore, the gap is $4 \times 45=225$ points. If the code sequence is $1-1$, the generated waveform is as shown in FigureA1.3. 


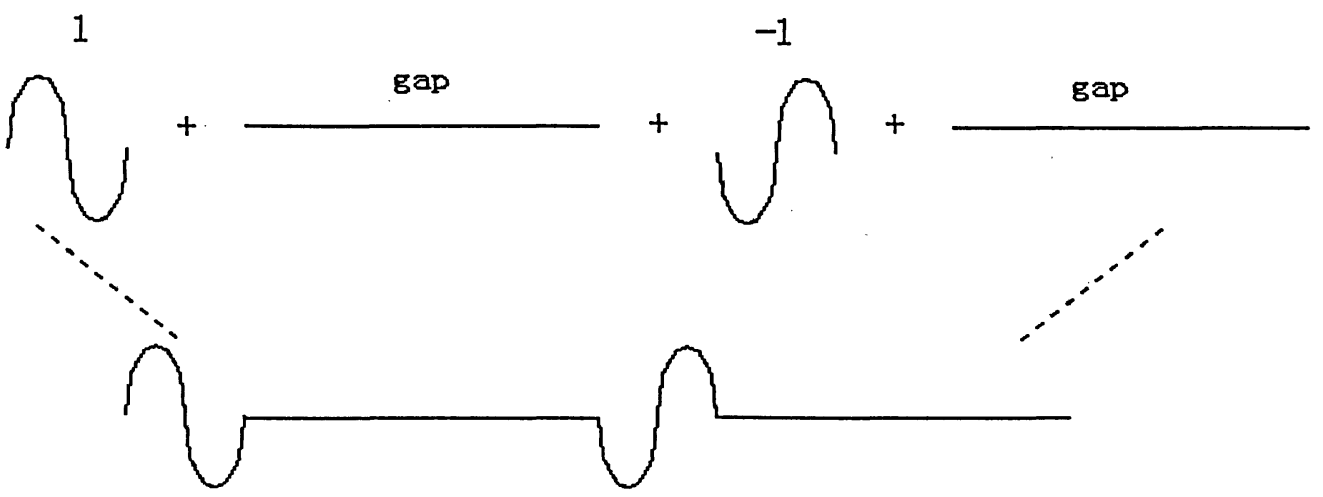

Figure A1.3 How to Generate the Encoded Signal

The equation editor was used to all the waveforms from various codes, in order to simplify the task. For example, the command join() was used to concatenate the shorter signals into a longer one. The rules used to generate the Golay code pairs are described in Section 2.2.1.2. The text file is then compiled into the waveform.

The signal is sent out based on the rules described as follows: 1) send out the signal; 2) wait for a time period of about $30-40$ micro-second to collect the backscatter signal (a blank waveform is used and the size is $(30 / 0.025) \times 45=$ 54000 points); 3) repeat this procedure, which is necessary for capturing and displaying the backscatter signal on screen of oscilloscope.

In the Sequence editor, we select the signal to be sent out and a time delay in the sequence list. And we also set an unconditional jump at the end of second waveform, which is then connected to the beginning of the first waveform. The Enhanced RUN mode is chosen to ensure the signal to be sent out in a continuous loop. 


\section{A1.2 Power amplifier ${ }^{[2]}$}

The main features of the power amplifier are listed below:

- Solid-state linear design

- Instantaneous ultra broadband

- Standard front panel manual gain adjust

- Suitable for CW/FM/PM/AM/Pulse/Digital modulations

- 50 Ohm Input/Output impedance

- High reliability and ruggedness

Table A1.2 Power amplifier SPECIFICATIONS

\begin{tabular}{|l|c|c|c|c|c|}
\hline \multicolumn{1}{|c|}{ Characteristics } & Rating & Min & Typ & Max & Units \\
\hline Frequency Response & BW & 0.01 & & 230 & $\mathrm{MHz}$ \\
\hline Output Power CW & $\mathrm{P}_{\text {Sat }}$ & 250 & 300 & & Watt \\
\hline Output Power @ 1dB G.C.P & $\mathrm{P}_{1 \mathrm{~dB}}$ & 150 & 200 & & $\mathrm{Watt}$ \\
\hline Input Power for Rated Output & $\mathrm{P}_{\text {in }}$ & & 0 & & $\mathrm{dBm}$ \\
\hline Power Gain @ 1dB G.C.P & $\mathrm{G}_{1 \mathrm{~dB}}$ & 54 & & & $\mathrm{~dB}$ \\
\hline Small Signal Gain Flatness & $\Delta \mathrm{G}$ & & & \pm 2.0 & $\mathrm{~dB}$ \\
\hline Gain Adjustment Range & FGA & 25 & 30 & & $\mathrm{~dB}$ \\
\hline Input/Output VSWR @ 50 ohm & S11/S22 & & $2.0: 1$ & & - \\
\hline Noise Figure & NF & & & 10 & $\mathrm{~dB}$ \\
\hline Harmonics @ 1dB G.C.P & H & & -20 & & $\mathrm{dBc}$ \\
\hline Third Order Intercept Point & IP3 & & +61 & & $\mathrm{dBm}$ \\
\hline Spurious Signals & Spur & & -70 & -60 & $\mathrm{dBc}$ \\
\hline Supply Voltage (single phase) & VAC & 100 & & 240 & Volt \\
\hline Power Consumption & Pd & & 1000 & 1200 & Watt \\
\hline
\end{tabular}

${ }^{[2]}$ Sources Empower RF systems, "Operating and service manual" 2003 
The important parameters are bandwidth, gain and impedance. The bandwiath, ranging from 0.01 to $230 \mathrm{MHz}$, is sufficiently wide to pass the center frequency $40 \mathrm{MHz}$ of the encoded signal. The gain is $54 \mathrm{~dB}$, and the adjustment range ranges from 25 to $30 \mathrm{~dB}$. The output of the arbitrary signal generator is $1 \mathrm{~V}$ and the transducer driver signal is about $160 \mathrm{~V}$. Therefore, the gain required is about $30 \mathrm{~dB}$. The impedance is $50 \mathrm{Ohm}$ and is the same as the transducer impedance. No matching circuit between the transducer and amplifier is needed, as discussed in Section 1.5.3.1.

\section{A1.3 Signal Amplifier ${ }^{[3]}$}

The gain, bandwidth, power supply and noise are important parameters for a signal amplifier. The type of amplifier we choose is integrated circuit RF amplifier. The advantages of this type of amplifier are listed as follows:

- High value of power gain are available, $40-70 \mathrm{~dB}$

- Internal feedback is minimized to the point where the circuits are usually unconditionally stable.

- Input impedance is not sensitive to load impedance.

- Internal limiter is optionally available.

The parameters of the signal amplifier are given in Table A1.3.

${ }^{[3]}$ Sources Miteq Inc, "Operating manual" 2003 
Table A1.3 Specifications of the Signal Amplifier

\begin{tabular}{|l|l|}
\hline Input/Output impedance & $50 \mathrm{ohm}$ \\
\hline Bandwidth $(\mathrm{MHz})$ & $1-500$ \\
\hline Gain & $60 \mathrm{~dB}$ Min, $61 \mathrm{~dB}$ Typ. \\
\hline Gain Flatness & $\pm 0.5 \mathrm{~dB}$ Max, $\pm 0.25 \mathrm{~dB}$ Typ. \\
\hline Noise Figure $(10 \mathrm{MHz})$ & $1.3 \mathrm{~dB}$ Max, $1.15 \mathrm{~dB}$ Typ. \\
\hline Noise Figure $(250 \mathrm{MHz})$ & $1.4 \mathrm{~dB}$ Max, $1.25 \mathrm{~dB}$ Typ. \\
\hline Noise Figure $(500 \mathrm{MHz})$ & $1.5 \mathrm{~dB}$ Max, $1.30 \mathrm{~dB}$ Typ. \\
\hline DC Power & $12-30 \mathrm{VDC} @ 95 \mathrm{~mA}$ \\
\hline
\end{tabular}

The bandwidth, gain and impedance are important parameters. The bandwidth, ranging from 1 to $500 \mathrm{MHz}$, is wide enough to pass the center frequency $40 \mathrm{MHz}$ of the encoded signal. The gain is $60 \mathrm{~dB}$. The output of the transducer is from 10 to 100 micro $\mathrm{V}$ and the output of the backscatter signal ranges from 5 to $20 \mathrm{mV}$; Therefore, the gain required is about $40-60 \mathrm{~dB}$. As can be seen, the gain of the chosen amplifier meets the requirement. The impedance is matched, as the amplifier is connected to the transducer directly.

\section{A1.4 Oscilloscope $\mathrm{e}^{[4]}$}

The parameters of the Oscilloscope are listed below:

- $500 \mathrm{MHz}$ Bandwidth Models

- 2 Channel Models

- 5 GS/s Maximum Real-time Sample Rate

- Up to 8 MB Record Length

- $100,000 \mathrm{wfms} / \mathrm{s}$ Maximum Waveform Capture Rate

- Intuitive User Interface for Easy Operation

- OpenChoice ${ }^{\mathrm{TM}}$ with Windows 2000 Delivers Built-in Networking and

${ }^{[4]}$ Sources Tektronix Inc. "TDS 5000 series digital phosphor Oscilloscopes user manual" 
Analysis

- Small Footprint

- 10.4 in. (264 mm) Bright Display

- Suite of Advanced Triggers

- CD-RW Drive Interoperability with Tektronix Logic Analyzers

- Two input impedance 50,1M Ohm.

The important parameters are the bandwidth, sample rate and record length and impedance. The bandwidth, $500 \mathrm{MHz}$, is wide enough to pass the center frequency $40 \mathrm{MHz}$ of the encoded signal. The sampling rate is $5 \mathrm{G}$, which is 100 times greater than the center frequency and is also greater than the signal generator output rate of $2.6 \mathrm{GHz}$. The recording length is $8 \mathrm{MB}$. If one sample needs 4 bytes to store, the number of total samples that the oscilloscope memory can save is $2 \mathrm{M}$ points. If the oscilloscope works at maximal sampling rate, the total time period it can store is $2 \mathrm{M} / 5 \mathrm{G}$ (about $0.4 \mathrm{~ms}$ ). This period is sufficient given our requirement. Backscatter signal is about $40-50 \mu$ s in length. Thus, the maximal length $8 \mathrm{MB}$ is sufficient. $50 \mathrm{Ohm}$ input impedance is ideal from impedance matching purpose

\section{A1.5 Filter Design}

Two filters, a high pass filter with $10 \mathrm{MHz}$ cutoff frequency and low pass filter with $100 \mathrm{MHz}$ cutoff frequency, are used before the signal entered the oscilloscope. There are three types of filters: Chebychev filter, Butterworth filter and Butterworth-Thompason filter. Chebychev family of filters ranges from a medium $\mathrm{Q}$ to a high $\mathrm{Q}$. Butterworth has only one design available and belongs to medium Q. Butterworth family of filters ranges from a lower $\mathrm{Q}$ to medium $\mathrm{Q}$.

The load Q dictates a number of filter properties. 
1. Low-Q filters generally have a flat, ripple-free passband, but their initial attenuation slope at the beginning of the stopband is low. Their phase response is close to linear with frequency, and therefore the delay is nearly constant across the passband.

2. High $\mathrm{Q}$ filters produce a faster initial slope in the stopband, and so have a greater attenuation at any stopband frequency than has a lower $\mathrm{Q}$ design with the same number of components. The penalty for this improvement is a passband attenuation ripple and poorer phase linearity.

3. The final attenuation slope and the final phase shift of any filter depend on the effective number of reactive components in the circuit.

4. The source and load resistances determine the overall $\mathrm{Q}$ of the filter and therefore the passband ripple, the phase linearity, and the exact cutoff frequency.

Since the frequency of our interest is around $40 \mathrm{MHz}$, we decide to choose the Chebychev family of filters, considering that their attenuation slopes are high at the beginning of the stopband. In order to get high slope, we choose the 7-element filter. From manual ${ }^{[5]}$, for high pass filter, we get the schematic graph which is shown in Figure A1.4. For the cutoff frequency $9.6 \mathrm{MHz}$, the values for various components are listed below: $\mathrm{C} 1$ and $\mathrm{C} 7$ are 470pf, L2 and L 6 are $0.618 \mathrm{uH}, \mathrm{C} 3$ and $\mathrm{C} 5$ are $200 \mathrm{pF}$ and $\mathrm{L} 4$ is $0.515 \mu \mathrm{H}$.

${ }^{[5]}$ Sources ARRL handbook for radio amateurs 


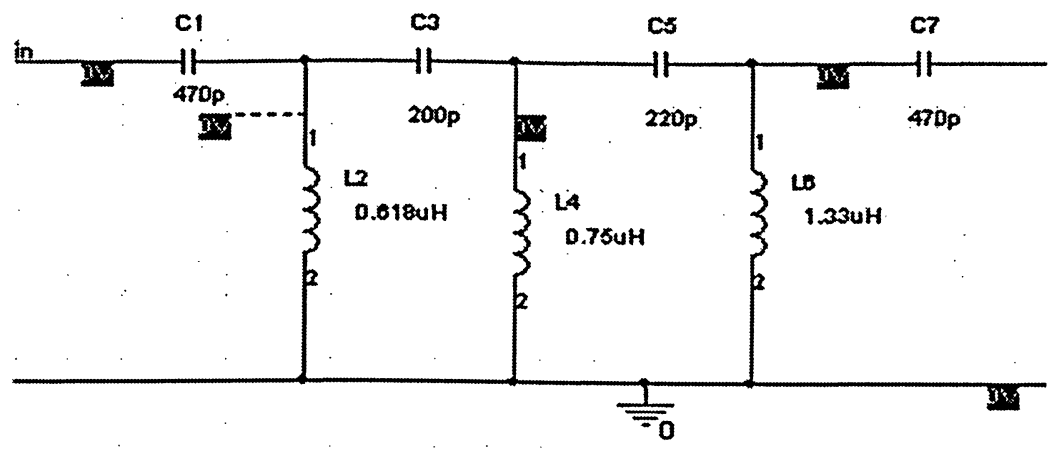

Figure A1.4 Schematic of High Pass Filter

The schematic of the low pass filter is shown in Figure A1.5. Given that the bandpass ripple is 0.1 , the load resistance is 1 , and the source is 1 , the cutoff frequency is $1 \mathrm{rad} / \mathrm{s}$. The values for various components are listed below. $\mathrm{C} 1$ and C7 are 1.198, L2 and L 6 are 1.444, C3 and C5 are 2.128, and L4 is 1.596. These values are scaled up to the terminating resistances and cutoff frequencies for our application by using equations a1.1 and a1.2.

$$
\begin{aligned}
& C=\frac{C_{n}}{2 \pi F_{c} R} \\
& L=\frac{R L_{n}}{2 \pi F_{c}}
\end{aligned}
$$

The values of $\mathrm{Cn}$ and $\mathrm{Ln}$ are obtained from the manual ${ }^{[6]} . \mathrm{R}$ is the load resistance of the final design. $\mathrm{Fc}$ is the $3 \mathrm{~dB}$ cutoff frequency.

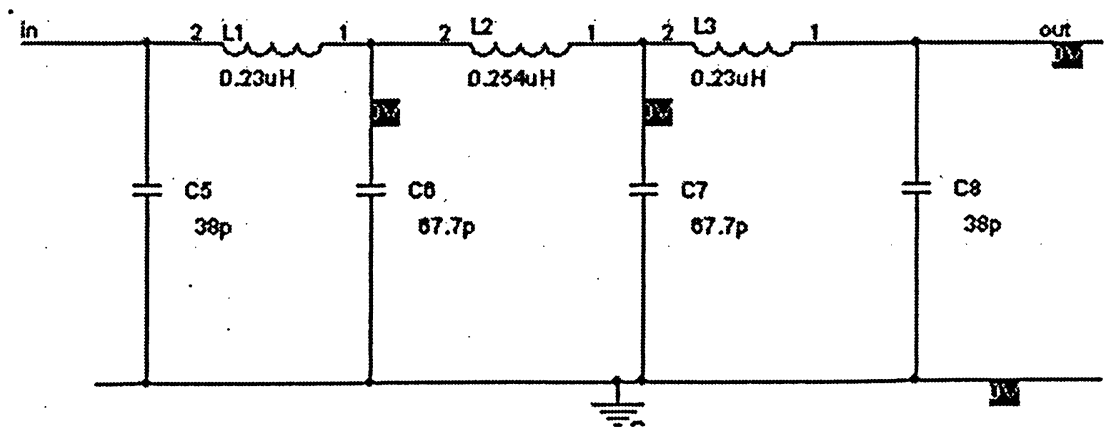

Figure A1.5 Schematic of Low Pass Filter

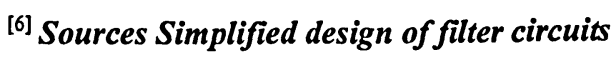


To match with the impedance of the cables and adapters, $R$ is chosen to be $50 \mathrm{ohm}$ and $\mathrm{Fc}$ is chosen to be $100 \mathrm{Mhz}$. Values for capacitors and inductors are listed below: $\mathrm{C} 7$ is $38 \mathrm{pF}, \mathrm{L} 2$ and $\mathrm{L} 6$ are $0.23 \mathrm{uH}, \mathrm{C} 3$ and $\mathrm{C} 5$ are $67.7 \mathrm{pF}$, and $\mathrm{L} 4$ is 0.254uH. The simulation results for these two filters are shown in Figure A1.6. The results demonstrate that the filter design is acceptable and the values of capacitors and inductors for these two filters are accurate.

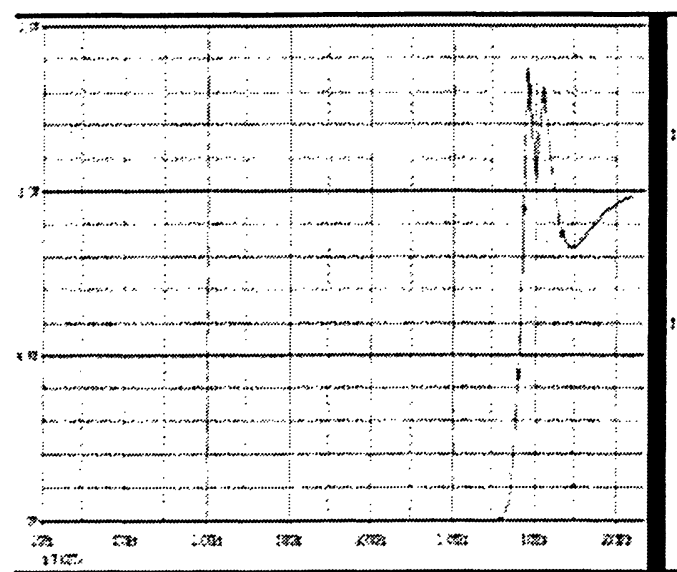

(a) high pass filter

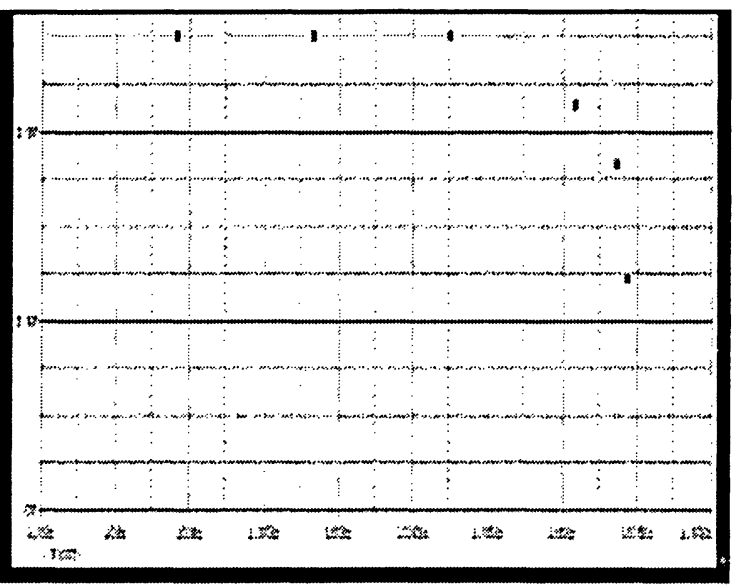

(b) low pass filter

Figure A1.6 Simulation Results of the Filters

OrCAD is used to design the PCB layout for the two filters, which is shown in Figure A1.7. In the PCB layout, the transmission line impedance matching is important. The capacitors we choose have high quality, and stable operation for high frequency circuits. The temperature coefficients are low, which is good for EMC. We choose surface mount package for capacitors and conductors to reduce coupled capacitance and inductance from the output leads so that we could improve the performance of our design for the high frequency range. Both filters should be placed in die cast box. 


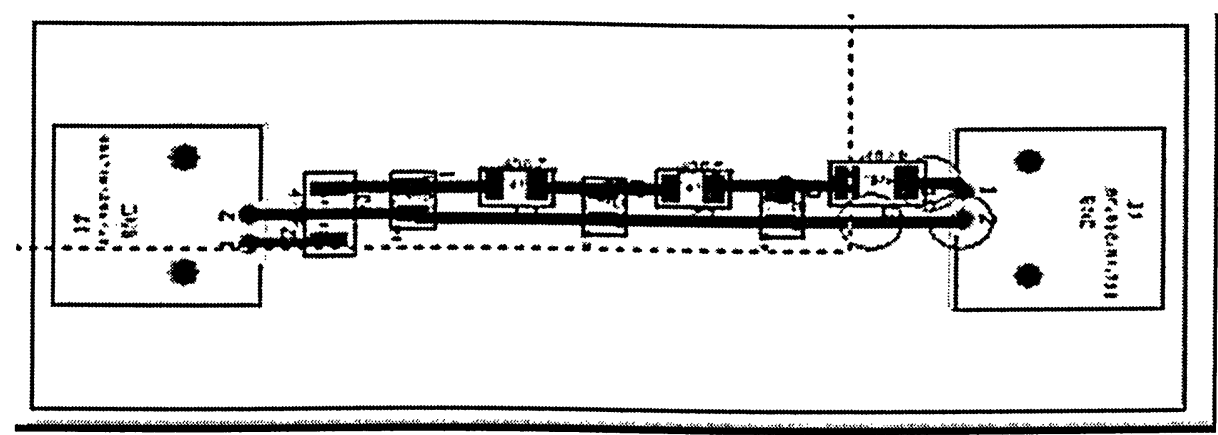

Figure A1.7 PCB Layout for Filters

\section{A1.6 Protection Circuit Design}

During the transmitting stage of the pulse echo sequence, the receiver amplifiers must be protected from the high voltage source. Also, during the receiving stage of the sequence, it is desirable to remove the high voltage source from the circuit to eliminate noise from the pulse electronics. Common solution to this problem employs crossed diode bridges. The crossed diodes act like switches that are closed for large signals, but are open for small signals. Using two pairs of crossed diodes and suitable cable length, a simple protection circuit is possible. One pair of crossed diodes is placed in series with the output of the pulser, while the other pair is placed in parallel with the input of the amplifier.

As shown in Figure A1.8, the protection circuit consists of two parts: the expander and limiter. An internal limiter is available from the manufacture, whereas the expander is made in house. The type of the diode is MMBD7000LT1. The diode is a dual switching diode, where two ports of the expander are used as the input and output. When the high voltage pulses are sent out from the source, one diode of the expander will conduct so that the source voltage passes unchanged. This signal will be short at the limiter so that the amplifier will be safe. During the receiving stage, the expander blocks the signal, which is small in strength (less than $0.7 \mathrm{v}$ ) and is directly connected with the amplifier. 


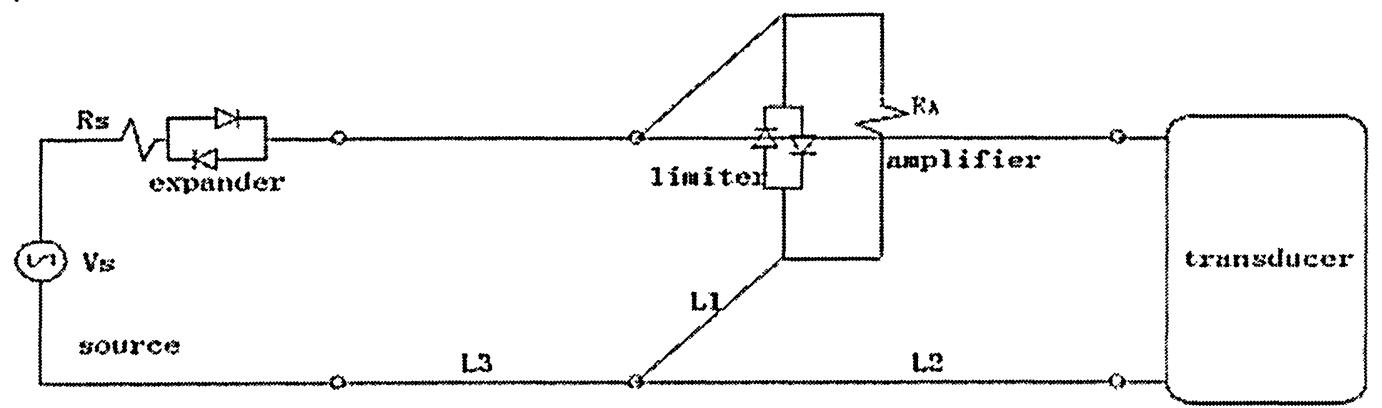

Figure A1.8 Protection Circuit ${ }^{[28]}$

The length of transmission lines, L1, L2 and L3, has an important effect on the frequency, amplitude and bandwidth of the entire system (article reference). L1 is the line from limiter to the T-junction. L2 is the line from transducer to T-junction. L3 is the line from expander to T-junction. The theoretical lengths of L1, L2 and L3 for the maximum pulse amplitude are listed in Table A1.4.

Table A1.4 The Line Lengths for Maximum Pulse Echo Amplitude ${ }^{[28]}$

\begin{tabular}{|l|c|c|c|}
\hline Frequency range $(\mathrm{MHz})$ & Length of L1 $(\mathrm{cm})$ & Length of L2 $(\mathrm{cm})$ & Length of L3(cm) \\
\hline $30-35$ & 80 & 100 & 100 \\
\hline $35-40$ & 160 & 100 & 80 \\
\hline $40-45$ & 140 & 100 & 60 \\
\hline $45-50$ & 120 & 100 & 160 \\
\hline
\end{tabular}

The schematic picture and PCB layout of the expander are shown in Figures A1.9 and A1.10. The transmission line is impedance matched. The expander is placed in a die cast box. 


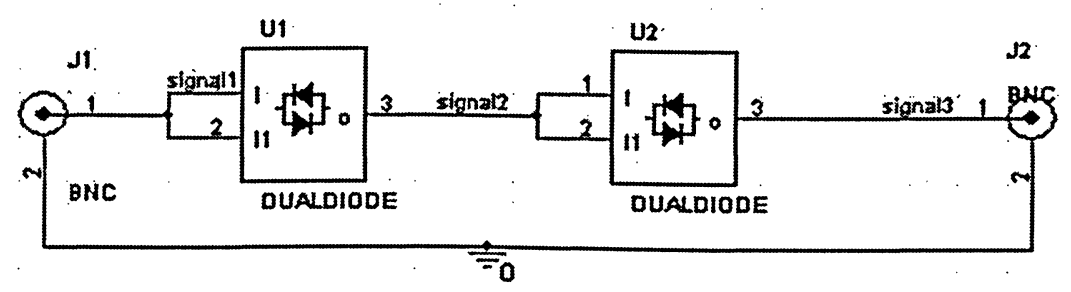

Figure A1.9 Schematic Picture of Expander

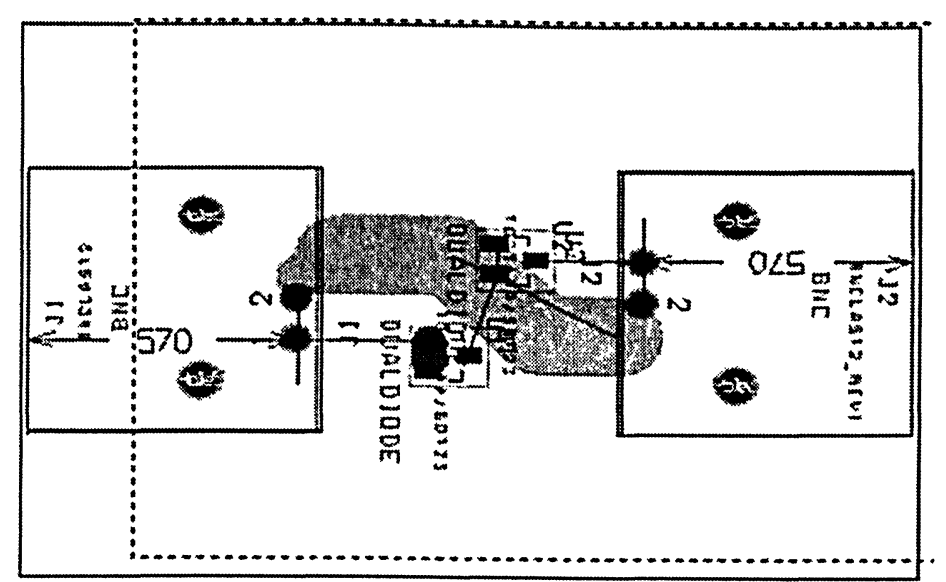

Figure A1.10 PCB Layout of Expander

\section{A1.7 Transmission Line Impedance Matching}

When operated at frequencies over $40 \mathrm{MHz}$, the traces on the printed circuit board are regarded as microstrip lines to use in impedance match. For our two-layer board as shown in Figure A1.11, the typical transmission line consists of one wide conductor on one side of the board and a ground plane on the other side of the board. 


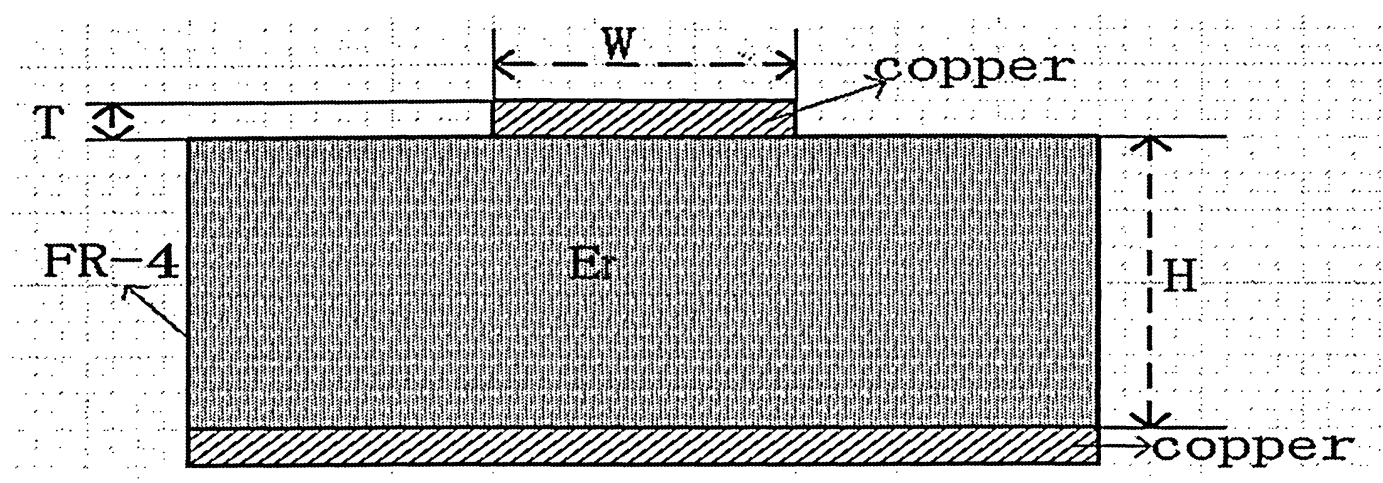

\section{Figure A1.11 Transmission Line ${ }^{[32]}$ \\ (Indicate the Conductor and the Ground Plane)}

Part of the electromagnetic wave travels in the air above the line, while the other part travels in the board material. Typical boards have a dielectric constant 2 to 10 times higher than that of the air, therefore the two parts of the wave travel at different speed along the line. Calculation of the exact impedance is difficult. The characteristic impedance of the line depends on the width of the upper conductor and the thickness and dielectric constant of the circuit board. The formula for estimating the impedance is listed below:

$$
Z=\left(\frac{87}{\sqrt{E r+1.414}}\right) \ln \left(\frac{5.98 H}{0.8 W+T}\right)
$$

where $\mathrm{Z}$ is the characteristic impedance, $\mathrm{W}$ is the width of the trace (in inch), $\mathrm{T}$ is the thickness of the trace (in inch), $\mathrm{H}$ is the distance between the signal trace and the reference ground (in inch), and Er is the dielectric constant of the planer material.

For our application, to match the trace impedance of the PCB board to other components, $Z$ should be tailored to $50 \mathrm{ohm}$. With $\mathrm{H}$ as $1.5 \mathrm{~mm}$ (about 0.06 inches), $\mathrm{T}$ is $0.1 \mathrm{~mm}$ (about 0.004 inches), and the dielectric constant of the material FR-4 is 4.7 , the width of trace is optimized as $2.5 \mathrm{~mm}$. 


\section{A1.8 Platform}

A platform is needed to support the tissue sample as well as the transducer. The platform should be able to move in three dimensions smoothly and accurately. A microscope was redesigned for this purpose. The light source of the microscope is replaced by a steel barwith a support frame clamped on it. The transducer, filters and the expander are fixed on the support to protect the cables from strong force. To study the depth properties of the binary-coded method, a vernier-scale label was added in the vertical direction as Figure A1.12.

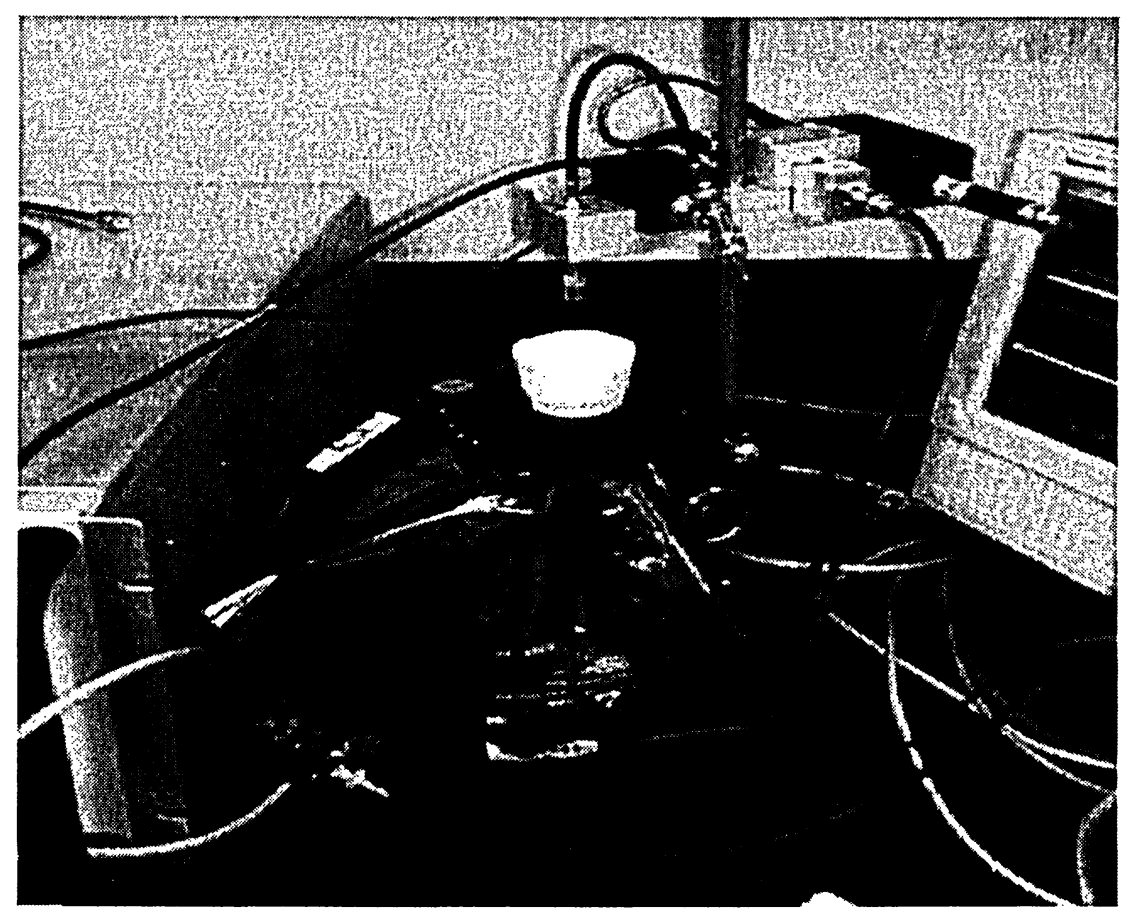

Figure A1.12 Lab Platform

\section{A1.9 Connection Adapter and Cables}

In Figure A1.13, several frames are chosen to represent the instruments that we have selected. The numbers within circle denote different types of connectors and 
adapters. In order to simplify the kinds of cables, only BNC-BNC (male to male) 50-ohm cables are used to connect all the instruments.

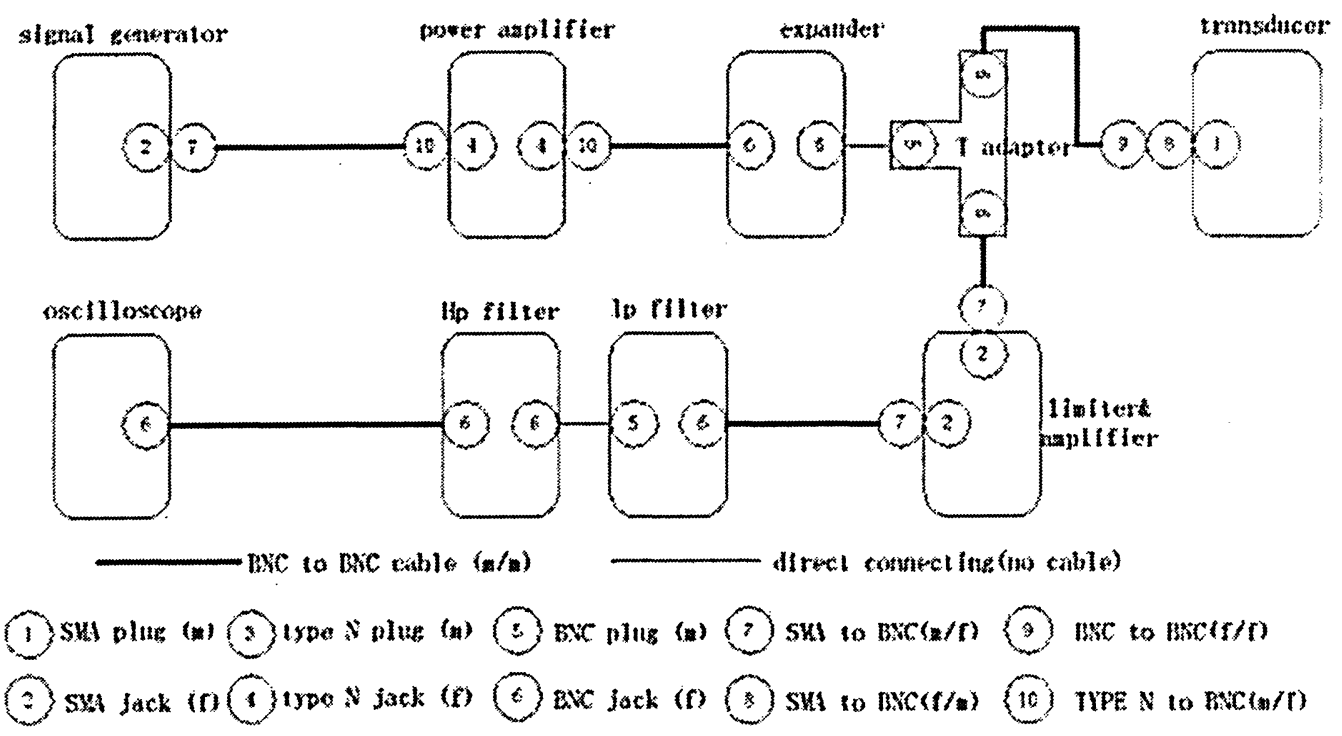

Figure A1.13 System Connection Diagram

Here is the list of all the adapters we need to use.

(all made by Pomona )

(7) SMA (male) to BNC (female) (m/f): Three. We have 2 already. Need to buy one more.

Cat. Number: 4290; Number: 1

8) SMA (female) to BNC (male) (f/m): one

9 BNC (female) to BNC (female) (f/f): Three. We have one and need to buy - two more.

Cat. Number: 3283; Number: 3

(10) TYPE N (male) to BNC (female) (m/f): we need to buy two.

Cat. Number: 3288; Number: 2 
6 pieces of cables are required. Considering the connectors of the boxes for the expander and the high-pass filter may need to be changed, we need to buy 9 pieces in total.

\section{A1.10 Two challenges of the lab system}

There are three challenges to be solved for our experiment system: the noise, the transducer inertia and the phase shift.

\section{A1.10.1 Noise}

This is the first problem we encounter and the most difficult problem to be solved. Noise is defined as any electrical signal presents in a circuit other than the desired signal. When we first tried to get the backscattered signal from the lab system, we can only see a lot of noise on the oscilloscope. The amplitude of noise is about $7 \mathrm{~V}$ from peak to peak.

Generally noise sources can be grouped into three major categories. The first is the so-called intrinsic noise, such as the thermal and shot noise in resistors and transistors that arises from random fluctuations. The second is the man-made noise, such as the noise generated by motors and switches. The third is due to natural disturbances, such as lightening and sun-spots.

The power consumption of the power amplifier is $1000-1200$ Watt. If the voltage is $110 \mathrm{~V}$, the current is about $11 \mathrm{~A}$. Such a heavy load sometimes makes the oil switch automatically switch off to avoid overheating. An exclusive power receptacle is later applied for the power amplifier. This method reduces the output noise from $7 \mathrm{~V}$ to $2 \mathrm{~V}$ peak-to-peak. However, $2 \mathrm{~V}$ peak-to-peak noise is still too 
large to identify the backscattered signals. Generally the backscattered signals from a tissue are only 5-20 milli-volt. Where does the noise come from?

Intuitively the noise should come from the power amplifier. We took a lot of time in doing experiment and discussing this problem with some experts. Another ultrasound system was borrowed from the Princess Margaret Hospital and compared to our system. There was no difference between the results generated through these two systems. We tried to use oscillating transformer to isolate the power supplies to the signal generator, the power amplifier, the signal amplifier and the oscilloscope. But there was no improvement for our results. We also added by-pass capacitors for the power supply of the signal amplifier. Some filters were put into the signal channel to filter the signal. When some metal boards and boxes were used to shield the components, there was still no improvement.

Finally, we find by increasing the number of serial connected diodes in the expander. The noise is dramatically reduced. This is due to the higher cutoff voltage from more diodes prevent the noise from the signal amplifier. More details are discussed in Appendix B.

\section{A1.10.2 Inertia of Transducer}

After the noise problems were solved, we got very good signals from real tissues when separate pulses were applied to the transducer. However, when we tried to send consecutive pulses to the transducer (as shown in Figure A1.14), no backscattered signals were observed. 


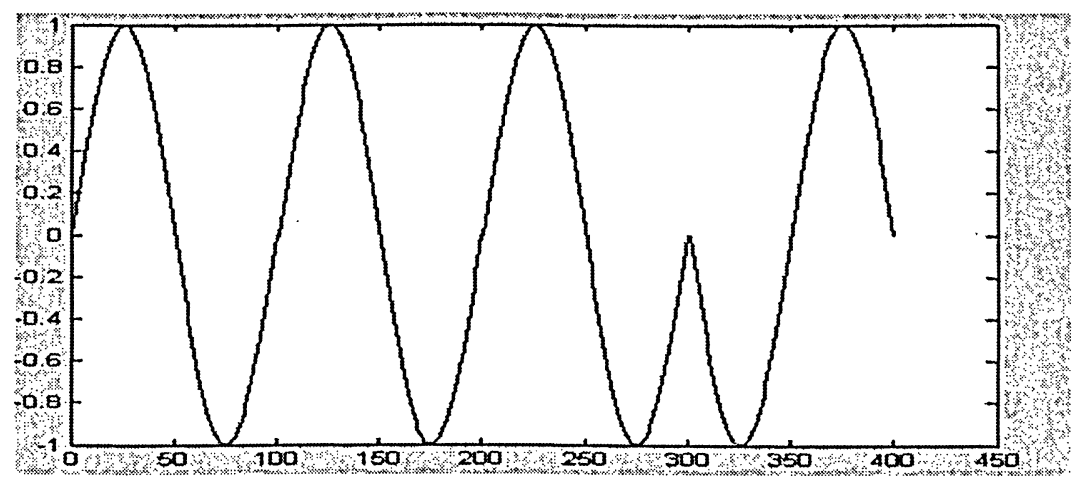

Figure A1.14 Consecutive Pulses

We suspected it was due to the inertia of the transducer, if the transducer needs some time to response to each pulse. We put some time gap between each pulse (as shown in Figure A1.15(a)). After this type of signal is sent to the transducer, very strong backscattered signals are received (Figure A1.15(b)).

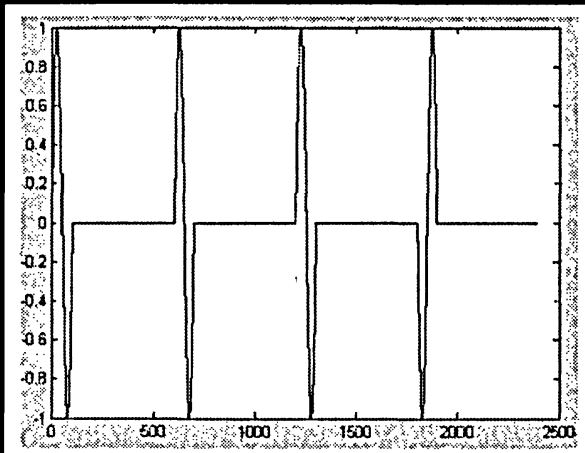

(a) Input pulser

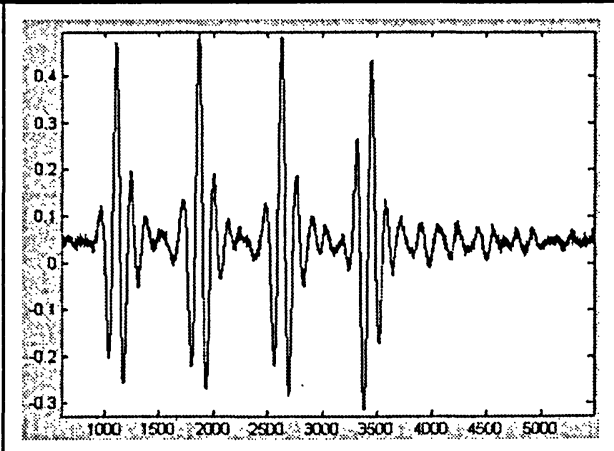

(b) Corresponding received reflection

Figure A1.15 New Pulser and Its Backscatter Signal

Another bad effect caused by the inertia of transducer is that several consecutive damping pulses are received even if only one pulse is sent to the transducer. As shown in Figure 1.15, each input pulse corresponds to several consecutive signals in the received signals. Furthermore, the shape of the output signal is changed due to the slow response. The inert properties of the transducer need to be improved. 


\section{Appendix B Noise reduction for the power amplifier}

In Section A1.10.1, we discuss the noise of our binary-coded excitation system. After serial connecting more diodes, the noise is successfully cut off from the power amplifier. In this section we optimize the number of diodes in the expander to keep the noise from the power amplifier in an acceptable range.

The diagram of the noise channel is shown in Figure B1.1. The noise from the power amplifier is shown as a signal source and its peak-to-peak amplitude is V1. The noise passes $\mathrm{n}$ serial connected diodes. If we assume a $0.7 \mathrm{~V}$ potential of the $\mathrm{PN}$ junction in each diode, the total potential for these $\mathrm{n}$ diodes is $\mathrm{n} \times 0.7$.

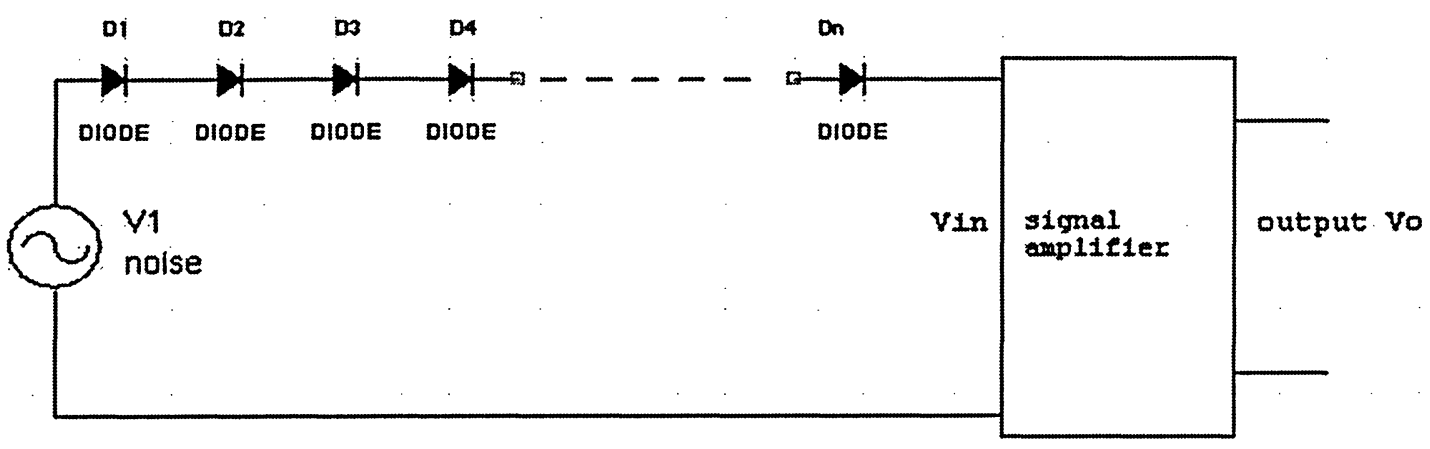

Figure B1.1 The Noise Channel

If the noise is large enough to pass these $\mathrm{n}$ diodes, Vin is the remainder noise sent into the signal amplifier and Vo corresponds to the noise output from the signal amplifier.

$$
\mathrm{Vin}=\mathrm{V} 1-\mathrm{n} \times 0.7
$$

The backscattered signal is about $20 \mathrm{mv}$ after the signal amplifier. However, the noise output after the signal amplifier is $2 \mathrm{~V}$. Therefore, the noise has to be significantly reduced before the signal amplifier. If the gain of the signal amplifier is $45 \mathrm{~dB}$, the noise before the signal amplifier has to be significantly less than $20 \times$ $10^{-45 / 20}=0.1125 \mathrm{mV}$.

From the electrical specification of the power amplifier, the maximal harmonics 
noise figure is $-20 \mathrm{~dB}$. The output from power amplifier is about $100 \mathrm{~V}$. Therefore, the amplitude of the noise is about $100 \times 10^{-20 / 20}=10 \mathrm{~V}$. So the number of diode should be $10 / 0.7=15$. In our expander we serially connect 12 diodes, which means the noise remains as $2.1 \mathrm{~V}$. 


\section{Appendix C Transducer Equivalent Circuit and Impedance matching}

\section{C1.1 Equivalent Circuit}

Numerous equivalent electrical circuits such as Mason's Equivalent Circuit, Simplified Equivalent Circuit and KLM Equivalent circuit ${ }^{[15]}$, have been proposed to model the electrical and mechanical characteristics of ultrasound transducers. In order to derive such a circuit, it is necessary to consider all the factors that exist in the practical situation. A thorough treatment of the problem is very complicated. Generally the radiation only takes place the surface of transducer. In Figure 1.2 (a), a four-terminal network with two electrical input terminals and two mechanical output terminals can be used to represent the transducer. At the output terminals a mechanical force $\mathrm{F}$ applied over the transducer face of area A produces particle motion of velocity v. For plane waves their ratio is the mechanical radiation resistance $Z_{R}$ given by

$$
Z_{R}=\frac{\text { force }}{\text { particle velocity }}=p A / v=\rho c A
$$

$\mathrm{Z}_{\mathrm{R}}$ is a pure resistance. If the transducer is tuned to resonance, the mechanical radiation resistance appears at the electrical terminals as a resistance $\mathrm{Re}$ given by

$$
R_{e}=Z_{R} / 4 N^{2}
$$

where $\mathrm{N}$ is the amplitude transformation ratio; the factor 4 from the doubling of velocity at the radiating face due to the low impedance at the opposite end. The equivalent circuit is shown in Figure1.2 (b), the simplification of the complete equivalent circuit is valid only at frequencies near resonance. 
(a)

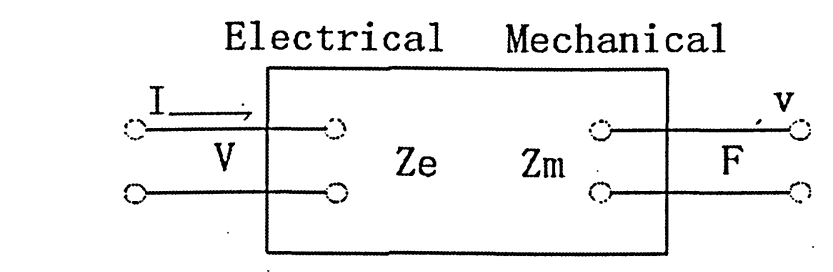

(b)
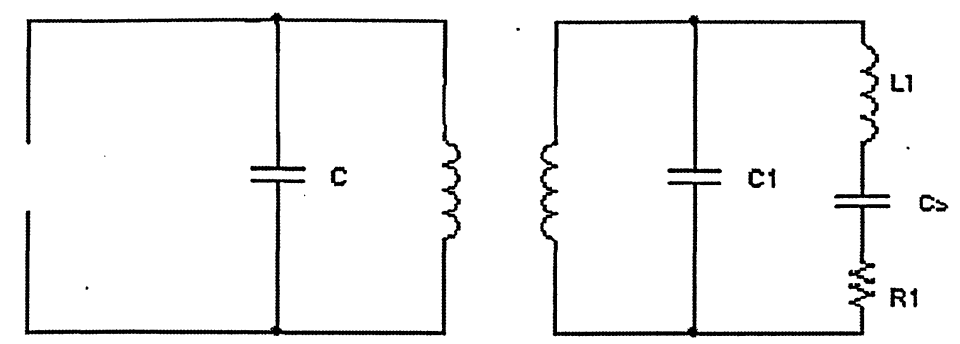

Figure C1.1 Equivalent Circuit of Transducer ${ }^{[15][53]}$

\section{$\begin{array}{lll}\text { (a) Four Terminal Network } & \text { (b) Equivalent Electrical Near Resonance }\end{array}$}

where $c_{1}=A \times \varepsilon / d \quad$ A is the area of the transducer, $\varepsilon$ is the dielectric constant of transducer, $d$ is the distance between the two electrodes.

$c_{s}=c_{1}\left(f_{p}^{2}-f_{s}^{2}\right) / f_{s}^{2}$ where $c_{s}$ is the capacitance of resonance, $\mathrm{f}_{\mathrm{p}}$ is the parallel or anti-resonant frequency, $f_{s}$ is the series or resonant frequency.

$L_{1}=1 / c_{s}\left(2 \pi f_{0}\right)^{2}$ where $L_{1}$ is the resonance inductance of transducer, $\mathrm{f}_{0}$ is the resonance frequency.

$R_{s}=1 /\left(2 \pi f_{0} c_{1} Q_{m}\right)^{2}$ where $\mathrm{R}_{\mathrm{s}}$ is the resistant load, $Q_{m}=1 /\left(2 \pi f_{s} R_{s}\right) \mathrm{Q}_{\mathrm{m}}$ is the mechanical quality factor ${ }^{[15]}$.

$\mathrm{fp}$ is parallel or anti-resonance frequency at which the impedance is a maximum. fs is the series or resonance frequency at which the impedance reaches a minimum. They are practical parameters that can be determined by measuring impedance ${ }^{[7]}$.

When a sinusoidal electrical driving signal is sent to a transducer, a corresponding variation in the thickness of the transducer is generated. The characteristic impedance at the boundary between the transducer and the medium determines the energy radiated at the face of the transducer. Some of the energy will be reflected back into the other face of transducer. The energy reflected at each face of the 
transducer travels back across the transducer towards the opposite face. Due to the electromechanical coupling, a new stress exists. The total stress is equal to the sum of all instantaneous values. Stresses at the same phases reinforce each other, which is known as resonance. The transducer also resonates when it is driven at frequencies at which the thickness of the transducer is equal to the odd integral number of half wavelengths.

\section{C1.2 Impedance Matching}

There are two separate problems in impedance matching: electrical impedance matching and mechanical impedance.

\section{C1.2.1 Electrical Impedance Matching}

In order to maximize the efficiency the electrical impedance of the transducer need to be matched to that of the receiver and driver circuits. This can be achieved by ensuring that the capacitance and inductance of the transducer form part of the tuned circuit of the associated electrical system. If the transducer is decided by application, the broad-band transformer can be used to match the impedance of the transducer to that of the driver circuit.

As Figure 1.3 illustrates, a transformer consists of two separate windings on some type of magnetic core. The core provides a path for the magnetic field that couples the two windings and also increases the winding inductance, which, along with the source and load resistances, determines the lower cutoff frequency. The impedance ratio will depend on the square of the turns ratio if the core can couple one winding to the other as equation (1.13). 


$$
\operatorname{Rin}=\left(\frac{n 1}{n 2}\right)^{2} \times \text { Rload }
$$
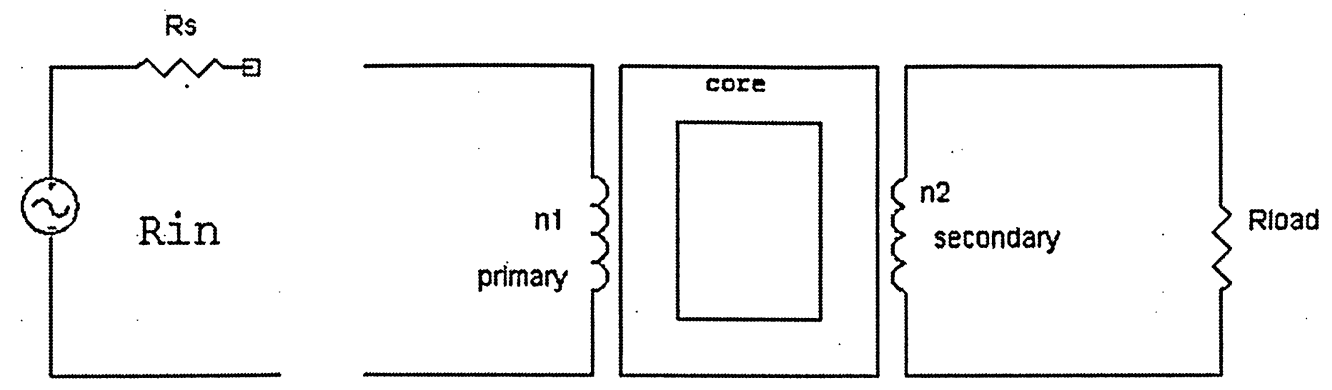

Figure C1.2 Transformers for Impedance Matching ${ }^{[14]}$

\section{C1.2.2 Mechanical Impedance Matching}

The effects of matching the characteristic impedance of the backing and loading media are important to ultrasound system. An impedance matching layers with appropriate thickness and characteristic impedance is often used between the surface of the transducer and the load. And the matching layer is generally chosen to be of quarter wavelength thickness, and of characteristic impedance equal to the geometric mean of those the transducer and of the loading medium. In biomedical application, water or other matching materials are usually used to match the mechanical impedance of transducer with the impedance of biological tissue. If the impedances of transducer and tissue do not match, most of the energy of ultrasound signal will be lost in the reflection ${ }^{[54]}$. 


\section{Appendix D Biological Effects}

\section{D1.1 Biological Effects}

Compared to X-ray, it is thought that ultrasound diagnosis is less harmful. But there are some side effects on biological tissue, even dangerous. In 1927, Wood and Loomis first did some experiments and described how small fish and frogs were killed during exposures of a few minutes to ultrasound at other frequency of $300 \mathrm{KHz}$ at an intensity of about $10 \mathrm{~W} / \mathrm{cm}^{-2}$ (much higher than that used for ultrasound imaging) Later people found other biological effects of ultrasound systems. A brief introduction follows.

In considering the biological effects of ultrasound, it is necessary to know the following physical parameters:
(a) The frequency of the wave;
(b) The total energy flux across the region of interest;
(c) The spatial distribution of the energy;
(d) The time distribution of the energy.

\section{D1.1.1 Thermal Effect}

Ultrasound is attenuated as it passes through tissues. The absorption mechanisms are discussed in section 1.6.3. The rate of absorption increases with frequency. Most of absorbed energy is converted into heat. These effects are dependent on the acoustic parameters of the incident beam and the biological target. A lot of experiments of exposure using different doses of energy, frequencies of ultrasound on different types of tissues are implemented to show different biological effects. For example, in studying frog skin, a well-known biological 
model for ion transport was used to investigate the biophysical effects of $(0.8,1.1$ and 3.3 MHz) ultrasound. It was found, as expected, that ultrasound induces both thermal and non-thermal effects. The non-thermal effect causes an increase in total ion conductance across the skin at $(0.8$ and $1.1 \mathrm{MHz})$ and a decrease at ( 3.3 $\mathrm{MHz}$ ). Furthermore, it was found that these effects are dependent on the pulse repetition rate and the duty cycle of the beam. Generally the effects will follow the laws as listed:

(1) Increasing time (contact time) will increase the probability of biologically significant thermal and cavitational effects.

(2) Increasing output power will increase the probability of biologically significant thermal and cavitational effects.

(3) Increasing transducer frequency increases thermal effects but decreases the probability of cavitational effects

\section{D1.1.2 Cavitation}

The term of cavitation is used to describe the behavior of gas bubbles in ultrasonic field. Acoustic cavitation is an effective mechanism for concentrating energy. It transforms the relatively low energy density of an ultrasonic field into the high energy density characteristic of the neighborhood and interior of a collapsing or vibrating bubble. There are two types of cavitations: transient and stable. Transient cavitation is the phenomenon in which voids suddenly grow from cavitation nuclei in the supporting liquid, and then collapse, under the influence of the changing pressure in an ultrasonic field: stable cavitation refers to the behavior of a gas filled bubble existing in an ultrasonic field of intensity below that necessary to cause transient cavitation. 
One of most important concepts in the cavitation research is cavitation threshold. The intensity of a given ultrasound signal is below a certain level, which is called cavitation threshold, cavitation does not occur. The numerical values of cavitation threshold depend on many variables such as the type of liquid, the size of distribution of nuclei, equilibrium pressure, temperature, vapor pressure, surface tension, the frequency and the duration of ultrasound signals.

It seems that ultrasound can cause the some damage. In order to avoid the damage happen, the intensity and time of ultrasound signals are limited by regulations. And the initial FDA (U. S. Food and Drug Administration) regulations on ultrasound output were published in 1976. These imposed application-specific limits, based on existing output levels that had demonstrated no adverse effects. Limits were divided into:

(1) Ophthalmic applications;

(2) Fetal and other (including abdominal, pediatric, small parts);

(3) Cardiac;

(4) Peripheral vessels. 


\section{Reference List}

[1] Braun, V. (1998) Dipulse-response Measurement of a Magnetic Recording Channel Using Golay Complementary Sequences, IEEE Transaction on Magnetics, Vol.34, No.1

[2] Brown, A. J. and Lockwood, R. G. (2002) Correspondence-A Low-cost, High-performance Pulse Generator for Ultrasound Imaging, IEEE Transactions on Ultrasonics, Ferroelectrics, and Frequency Control, Vol.49, No.6

[3] Chiao, Y. R. and Hao, X. (2003) Coded Excitation for Diagnostic Ultrasound: A System Developer's Perspective, Preprint for 2003 IEEE Ultrasonics Symposium

[4] Chiao, Y. R. and Lewis, J. T. (2000) Synthetic Transmit Aperture Imaging Using Orthogonal Golay Coded Excitation, IEEE Ultrasonics Symposium, pp.1677-1680

[5] Chiao, Y. R., Mo, Y. L., Hall, L. A., and Miller, C. S. (2000) B-Mode Blood Flow (B-Flow) Imaging, IEEE Ultrasonics Symposium, pp.1469-1472

[6] Cobbold, S. C. R. (2003) Foundations of Biomedical Ultrasound, Institute of biomaterials \& Biomedical Engineering, Working Paper, University of Toronto

[7] Ding, X. Z. and Payne, A. P. (1990) A New Golay Code System for Ultrasonic Pulse-echo Measurements, Meas. Sci. Technol., Vol.1, pp.158-165

[8] Duck, A. F., Baker, C. A. and Strritt, H. C. $\{1998\}$ Ultrasound in Medicine, Institute of Physics Publishing Bristol and Philadelphia

[9] Ebbini, S. E. and Shen, J. P. (1993) A New SVD-Based Optimal Inverse Filter Design for Ultrasonic Applications, Ultrasonics Symposium, pp.1187-1190

[10] Ebbini, S. E. and Shen, J. P. (1997) Fundamental Resolution Limits of a Coded-excitation System for Real-time Pulse-echo Imaging, IEEE Ultrasonic Symposium, pp.1539-1542

[11] Foster, S. (1986) Impulse Response Measurement Using Golay Codes, IEEE ICASSP 86, Tokyo, pp.929-932

[12] Fry, J. F. (1978) Methods and Phenomena-ultrasound: Its Application in Medicine And Biology, Elsevier scientific publishing company, Amsterdam. Oxford. New York

[13] Golay, J. E. M. (1961) Complementary Series, IRE Transactions of Information Theory 
Vol.7, pp.82-87

[14] Hardy, J. (1979) High Frequency Circuit Design, Preston Publishing Co, Reston Virginia

[15] Hunt, W. J., Ardit, M., and Foster, F. S. (1983) Ultrasound Transducers for Pulse-echo Medical Imaging, IEEE Transactions on Biomedical Engineering, Vol. BME-30, No. 8

[16] Jain, K. A. (1989) Fundamental of Digital Imaging Processing, Prentice Hall, Inc. Englewood Cliffs, New Jersey

[17] Jensen, A. J. (1996) Estimation of Blood Velocities Using Ultrasound: A Signal Processing Approach, Cambridge University Press

[18] Jensen, A. J. (1997) A New Approach to Calculating Spatial Impulse Responses, IEEE Ultrasonics Symposium, pp.1755-1759

[19] Jensen, A. J., Holm, O., Jensen, J. L., Bendsen, H., and Pedersen, M. H. (1999) Experimental Ultrasound System for Real Time Synthetic Imaging, IEEE Ultrasonic Symposium, Vol.2, pp.1595-1599

[20] Jeong, K. M. and Ebbini, S. E. (2000) Optimal Transmit/receive Beam Synthesis for Coded Excitation Pulse-echo Imaging, IEEE Ultrasonics Symposium, pp.1219-1222

[21] Kolios, M. (2001) High Frequency Ultrasound Imaging and Spectroscopy for Monitoring Apoptosis, Biomedical Engineering Research Grant

[22] Kolios, C. M., Taggart, L., Baddour, E. R., Foster, S. F., Hunt, W. J., and Czarnotal, J. G. (2003) An Investigation of Backscatter Power Spectra from Cells, Cell Pellets and Microspheres, IEEE Ultrasonics Symposium proceedings, pp.752-757

[23] Kossoff, G. (1979) Analysis of Focusing Action of Spherically Curved Transduce, Ultrasound Med. Biol., Vol.5, pp.359-369

[24] Kudo, N., Kamataki, T., Yamamot, K., Onozuka, H., and Mikami, T. (1997) Ultrasound Attenuation Measurement of Tissue in Frequency Range 2.5- $40 \mathrm{MHz}$ Using a Multi-resource Transducer, IEEE Ultrasonic Symposium, pp.1181-1184

[25] Lee B. B. and Furgason, E. S. (1981) A New Digital Correlation Flaw Detection System", J. Non Destructive Testing, Vol.2, pp.57-63

[26] Li, P. (1999) Pulse Compression for Finite Amplitude Distortion Based Harmonic Imaging Coded Waveforms, Ultrasonic Imaging, Vol.21, pp.1-16

[27] Li, P., Ebbini, E., and O’Donnell, M. (1992) A New Filter Design Technique for Coded 
Excitation Systems, IEEE Transactions on Ultrasonics, Ferroelectrics, and Frequency Control, Vol.39, No.6

[28] Lockwood, R. G., Hunt, W. J., and Foster, F. S. (1991) The Design of Protection Circuitry for High Frequency Ultrasound Imaging Systems, IEEE Transactions on Uitrasonics, Ferroelectrics, and Frequency Control, Vol.38, No.1

[29] Misaridis, X. T., Gammelmark, K., and Jorgensen, H. C. (2000) Potential of Coded Excitation in Medical Ultrasound Imaging, Ultrasonics, Vol.38, pp.183-189

[30] Misaridis, X. T. and Jensen, A. J. (1999) An Effective Coded Excitation Scheme Based on a Predistorted FM Signal and an Optimized Digital Filter, IEEE Ultrasonics Symposium, pp.1589-1593

[31] Misaridis, X. T. and Pedersen, H. M. (2000) Clinical Use and Evaluation of Coded Excitation in B Mode Images, IEEE Ultrasonics Symposium, pp.1689-1693

[32] Montrose, I. M. (1996) Printed Circuit Board Design Techniques for EMC Compliance, IEEE Press. New York

[33] Narayanan, V. M., Molthen, C. R., and Shankar, M. P. (1997) Studies on Ultrasonic Scattering from Quasi-periodic Structures, IEEE Transactions on Ultrasonics, Ferroelectrics, and Frequency Control, Vol.44, No.1

[34] O' Donnell, M. (1992) Coded Excitation Systems for Improving the Penetration of Real-time Phased-array Imaging Systems, IEEE Transactions on Ultrasonics, Ferroelectrics, and Frequency Control, Vol. 39, No. 3

[35] O’Neil, T. H. (1949) Theory of Focusing Radiators, J. Acoust. Soc. Amer., Vol.21, pp.126-516

[36] Oppenheim, V. A., Willsky, S. A., and Hawab, S. H. (1997) Signals \& Systems, Prentice-hall

[37] Parker, K.J. (1983) Ultrasonic Attenuation and Absorption in Liver Tissue, Ultrasound Med. Bio., Vol.9, pp. 363-369

[38] Raju, I. B. and Srinivasan, A. M. (2002) Statistics of Envelope of High-frequency Ultrasonic Backscatter from Human Skin in Vivo, IEEE Transaction on Ultrasonics, Ferroelectrics, and Frequency Control, Vol.49, No.7

[39] Rao, N. (1990) Frequency Modulated Pulse for Ultrasonic Imaging in an Attenuating 
Medium, IEEE Symposium on Computer Based Medical Systems

[40] Rao, N. (1994) Investigation of a Pulse Compression Technique for Medical Ultrasound: a Simulation study, Medical \& Biological Engineering \& Computing

[41] Rao, N. and Aubry, M. (1994) Evaluation of a Pulse Compression Technique for Ultrasound Speckle Reduction, IEEE Trans. Ultrason. Feerroelec. Freq. Control, Vol.41, pp.660-663

[42] Rao, N. and Mehra, S. (1993) Medical Ultrasound Imaging Using Pulse Compression, Electronics Letters, Vol.29, pp.649-651

[43] Rao, N., Mehra, S., Bridge, J., and Venkatraman, S. (1995) Experimental Point Spread Function of FM Pulse Imaging Scheme, Ultrasound Imaging, Vol.17, pp.114-141

[44] Schroeder, R. M. (1979) Integrated Impulse Method Measuring Sound Decay without Using Impulse, J. Acoust. Soc. Am. Vol.66, pp.497-500

[45] Shen, J. and Ebbini, S. E. (1996) A New Coded Excitation Ultrasound Imaging Systems, IEEE Transactions on Ultrasonics, Ferroelectrics, and Frequency Control, Vol. 43, No.1

[46] Shen, J. and Simon, C. (1996) A New Pulse-echo System for Real Time 3D Ultrasonic Imaging-phantom Tests, IEEE Ultrasonics Symposium, pp.1387-1390

[47] Shen, J. and Ebbini, S. E. (1996) On the Design of a Transversal Filter Bank for Parallel Processing Multiple Imaging Lines in Real-time Acoustic Imaging, IEEE, pp.3109-3112

[48] Shen, J. and Ebbini, S. E. (1998) Filter-based Coded-excitation System for High-speed Ultrasonic Imaging, IEEE Transactions on Medical Imaging, Vol.17, No.6, pp.923-934

[49] Shen, J., Erdogan, H., and Ebbini, S. E. (1994) An Optimal Imaging Operator Design Technique for Coded Excitation Ultrasound Imaging System, IEEE Ultrasonics Symposium, pp.1777-1781

[50] Shung, K. K. and Thieme, A. G. (1993) Ultrasonic Scattering in Biological Tissues, CRC Press, Boca Raton. Ann Arbor. London. Tokyo

[51] Sklar, B. (2001) Digital Communications Fundamentals and Application, second edition, Prentice-hall

[52] Takeuchi, Y. (1979) An Investigation of a Spread Energy Method for Medical Ultrasound System, Ultrason., Vol.17, pp.175

[53] Wells, P. N. T. (1977) Biomedical Ulrtrasonics, Academic Press, London. New York. San 
Francisco

[54] Wilson, S. G. (2001) Digital Modulation and Coding, Prentice-hall International

[55] Yokosawa, K. and Shinomura, R. (1996) A 120-MHz Ultrasound Probe for Tissue Imaging, Ultrasonic Imaging, Vol.18, pp.231-239

[56] Zhou, B. and Green, M. D. (1992) Characterization of External Ear Impulse Response Using Golay Code, J. Acoust. Soc. Am. Vol.92, pp.1169-1171 


\section{Important Glossary}

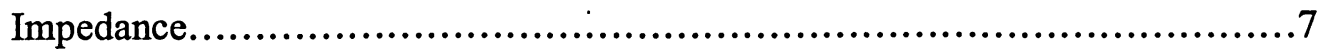

Intensity ..................................................................... 10

One cycle excitation backscatter signal or one cycle excitation...................24

uni-polar modulated method.......................................................34

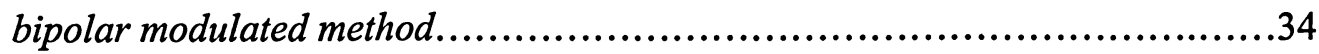

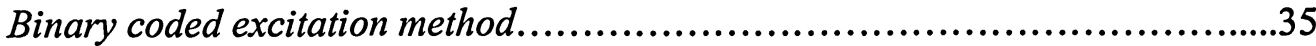

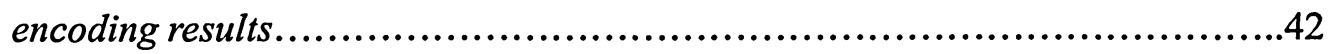

reflection reference processing method.........................................60

code reference processing method................................................61

one-cycle code reference..........................................................61

three-cycle code reference. ........................................................61

code with phase reference processing method...................................62

one-cycle code with phase reference method .......................................63

three-cycle code with phase reference method.................................63 
Abbreviations

FM Frequency Modulated

SNR Signal to Noise Ratio

US Ultrasound

PZT lead zirconate titanate

PVDF Polyvinlidene difluoride

A/D Anolog to Digital converter

NDE non-destructive testing

FD Frequency Dependent 